Under the label of 'Net recommendation', the present study aims to offer a balanced and constructive normative analysis of digital media, focusing on the relations between digital

media and the good life. The project of Net recommendation aims to (re)assert the importance of actual discourses in our normative analysis of the relations between digital media and the good life. I pursue this project with a Walzerian approach to digital media and the good life that takes seriously (and, ideally, also interacts with) actual discourses. This approach allows us to have a better understanding of our normative judgements on the impacts of digital media has (or will have) on the good life and, at the same time, allows us to answer the question of 'how should we live with digital media?' more adequately. Moreover, the Walzerian approach is useful in both an intra-cultural and an inter-cultural context. Using China's Internet as a case study, I show that the relations between digital media and the good life in each culture should be examined in its own right.

Together, I hope, this study provides an adequate illustration and defence of the project of $\mathrm{Net}$ recommendation that does not start with the assumption of digital media being a source of moral prom and strives to recommending specific ways to reform and/or transform digita media and digitally-mediated practices that allow us to have better relations with digital media and enable us to live better lives with them.

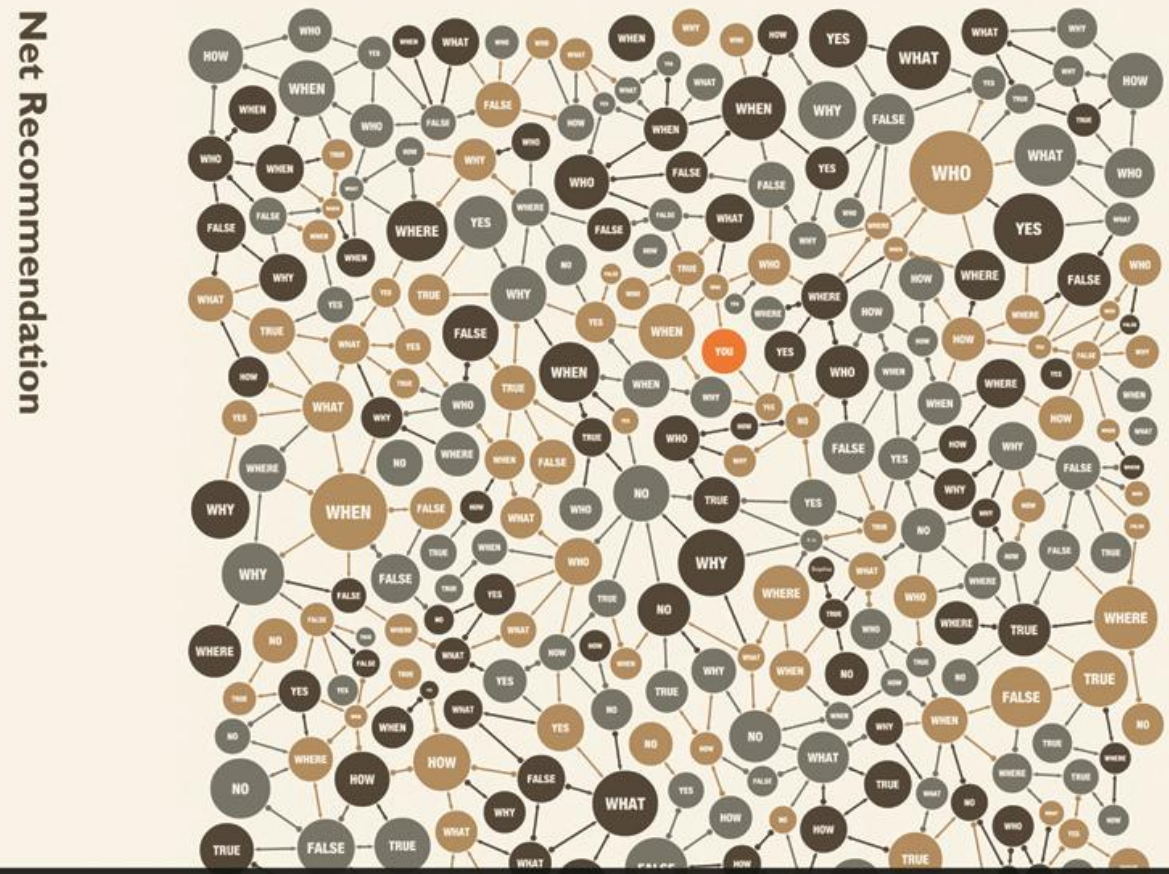

\title{
Net Recommendation
}

\section{Prudential Appraisals of Digital Media} and the Good Life 


\section{Net Recommendation}

Prudential Appraisals of Digital Media and the Good Life 
Promotion Committee:

Rector Magnificus, voorzitter

Prof. dr. P.A.E. Brey, University of Twente, promotor

Prof. dr. V. Evers, University of Twente

Prof. dr. H.F.M te Molder, University of Twente

Prof. dr. J.A.M. Bransen, Radboud University Nijmegen

Prof. dr. C.M. Ess, Aarhus University

Prof. dr. B.C. Stahl, De Montfort University

Printed by: Wöhrmann Print Service, Zutphen, The Netherlands

Cover image: Q \& A (at http://flic.kr/p/6VWcxe/) by @ Mario Klingemann Cover design by: Pak-Hang Wong

(C) Pak Hang Wong, 2012

All rights reserved. No part of this publication may be reproduced, stored in a retrieval system, or transmitted, in any form or by any means, without prior written permission of the author. 


\section{NET RECOMMENDATION}

PRUDENTIAL APPRAISALS OF DIGITAL MEDIA AND THE GOOD LIFE

\section{DISSERTATION}

to obtain

the degree of doctor at the University of Twente, on the authority of the rector magnificus,

Prof. dr. H. Brinksma,

on account of the decision of the graduation committee,

to be publicly defended

on Friday the $8^{\text {th }}$ June 2012 at 16.45 hrs

by

Pak Hang Wong

Born on $9^{\text {th }}$ June, 1980

in Hong Kong, China 
This dissertation has been approved by promotor:

Prof. dr. P.A.E. Brey

(C) Pak Hang Wong, 2012

ISBN: 978-90-365-3360-7 
For Suet Yi 



\section{CONTENTS}

\section{CONTENTS}

INTRODUCTION Prologue to Net Recommendation 1

I Ethics, the Good Life and Digital Media 4

II An Overview of the Dissertation $\quad 8$

CHAPTER ONe Appraising Digital Media and the Good Life 13

1.1 A Walzerian Approach to Digital media and the Good Life 15

1.2 What is an Appraisal of Digital Media? 25

1.3 A Taxonomy of the Appraisals of Digital Media 26

$\begin{array}{ll}1.4 & \text { On Prudential Appraisals } \\ 1.5 & 28\end{array}$

1.5 Prudential Appraisals and the Good Life 37

1.6 Why Do We Need Prudential Appraisals of Digital media? 51

1.7 Conclusion $\quad 59$

CHAPTER Two Modern Identity and the Good Life $\quad 61$

2.1 Modernity and Modern Identity $\quad 63$

2.2 Taylor on Modern Identity $\quad 65$

2.3 Late Modern Societies and Self-Identity: Giddens and Beck et al. 74

2.4 Living a Good Life in Modern and Late Modern Societies 84

$\begin{array}{ll}2.5 \text { Conclusion } & 90\end{array}$

ChAPTER Three From Culture 2.0 to a Network State of Mind 93

3.1 A Short Introduction to Web 2.0 95

3.2 Enemies and Friends of Culture 2.0 98

3.3 Vices and Virtues of a Network State of Mind 106

3.4 Who Should We Be in a Digitally-Mediated World? 114

3.5 Conclusion 123 
ii |

CHAPTER FOUR Another Look at the Good Life 125

$\begin{array}{lll}4.1 & \text { From Singular Modernity to Plural Modernities } & 127\end{array}$

4.2 Confucian Dao, Harmony and Personhood 134

4.3 Confucian Ethics: The Basics 144

4.4 On the Confucian Way of Life: A Contemporary Account 149

4.5 Conclusion 160

CHAPTER FIVE The Making of China's Internet $\quad 161$

5.1 The Chinese Communist Party and the Internet 163

$\begin{array}{ll}5.2 \text { Governing China's Internet } & 164\end{array}$

5.3 China's Internet for the Public $\quad 171$

5.4 China's Internet and Confucianism: A Walzerian Perspective 178

5.5 Conclusion 194

ChAPTER SIX Digital Media, Recommendation and Design 197

6.1 Perils of Recommendation 199

6.2 Paternalism is Inevitable: An Inconvenient Truth 204

6.3 On Being a 'Paternalistic' Philosopher 208

$\begin{array}{ll}6.4 \text { Conclusion } & 212\end{array}$

$\begin{array}{ll}\text { EPILOGUE } & \mathbf{2 1 5}\end{array}$

$\begin{array}{ll}\text { SUMMARY } & 221\end{array}$

$\begin{array}{ll}\text { SAMENVATTING } & 225\end{array}$

$\begin{array}{ll}\text { BIBLIOGRAPHY } & 231\end{array}$ 


\section{ACKNOWLEDGEMENTS}

The Oxford English Dictionary defines a dissertation as: "a spoken or written discourse upon or treatment of a subject, in which it is discussed at length; a treatise, sermon, or the like", specifically it is "an extended scholarly essay, usu. based upon original research, submitted for a degree or other academic qualification". The OED is certainly correct (by definition, of course). However, to me, a $\mathrm{PhD}$ student, a dissertation means for a lot more than "an extended scholarly essay [...] submitted for a degree or other academic qualification", it is also a memento of a journey filled with interesting characters and amazing stories. I arrived at the University of Twente in the summer of 2008, knowing no one in Enschede or the Netherlands, knowing practically nothing about philosophy and ethics of technology. Now, I have completed a $\mathrm{PhD}$ dissertation in this field, and I have met, befriended, and learned from many people in Enschede and around the world. For me, this has truly been an incredible journey.

This journey would not be possible without my promotor and supervisor Philip Brey, who offered me the opportunity to work in the NWO VICI research project "Evaluating the Cultural Quality of New Media". I would also like to thank him for his guidance, understanding, and support. Like any good supervisor, he has challenged and criticised the ideas and arguments presented in this dissertation in order to improve them. I am sure that he still disagrees with many of my ideas and arguments, but I am also sure that he has left enough from for reasonable disagreement.

It is also my pleasure to have worked with a wonderful group of philosophers in the research project and in Department of Philosophy at University from whom I have learned so much. I must admit that I came here as an 'analytic' philosopher and disregarded other philosophical traditions, but you have all taught me to appreciate different ways of 'doing' philosophy. My special thanks go to Adam Briggle, Mark Coeckelbergh, Ed Spence and Johnny Søraker, and Aimee van Wynsberghe who have read and commented on bits and pieces of my work in numerous meetings. My thanks also go out to my fellow (ex-) PhD students Aimee, 
Anna Laura, Dirk, Federica, Fred, Govert, Johnny, Litska, Lucie, and Steven, without whom this journey would not be half as enjoyable. I only wish there were more conferences and workshops we could go to together. Good times, indeed.

I have presented my ideas and arguments at various conferences and workshops, and have submitted my work to different journals. I want to thank all the people I have met in conferences and workshops and the anonymous reviewers for providing useful comments, suggestions and encouragement. Particularly, I want to thank Charles Ess and Rafael Capurro for inspiring me to work on intercultural (and cross-cultural) information ethics, which forms a substantial part of this dissertation.

Also, I would like to express my gratitude to Emiliano Eduard Heyns and Gijs Houwen for their translation of the Dutch Summary, and to Scott Robbins for his proofreading of the manuscript.

Finally, I want to thank my family, especially you Sophia, for supporting my decision to leave Hong Kong and to pursue my dream: Philosophy. Sophia, I know it has been difficult for you, but I hope this journey has brought us closer. I probably should say something splendid, but I know my words will fail me here. So, thank you, my dear. And, as I have promised, this book is for you.

Pak Hang Wong 
Parts of this dissertation have used materials that are published. Specifically,

INTRODUCTION used material from "The 'Good Life' in Intercultural Information Ethics: A New Agenda”, published in International Review of Information Ethics, 13, 26-32.

The final publication is available at: www.i-r-i-e.net.

CHAPTER ONE is published in an abridged version as "A Walzerian Approach to ICTs and the Good Life" in Journal of Information, Communication and Ethics in Society, 10 (1), 19 35. The final publication is available at: www.emeraldinsight.com/jices.htm.

CHAPTER Four (esp. section 4.2, 4.3) used material from "Dao, Harmony and Personhood: Towards a Confucian Ethics of Technology", published in Philosophy \& Technology, 25 (1), 67-86. The final publication is available at: www.springerlink.com.

CHAPTER SIX is published, with modifications, as "Technology, Recommendation and Design: On Being a 'Paternalistic' Philosopher”, in Science and Engineering Ethics.

The final publication is available at: www.springerlink.com. 



\section{INTRODUCTION}

\section{Prologue to Net Recommendation}

Digital media has become an integral part of people's lives (at least, for those who live in the developed world), and its ubiquity and pervasiveness in our everyday lives raise new ethical, social, cultural, political, economic and legal issues, which have attracted researchers from various fields to study digital media and its impacts more closely. Particularly, the rise of digital media has led to the formation of a subfield of ethics now generally known as Information and Computer Ethics (ICE). ${ }^{1}$ Researchers in the field of ICE have already taken up various issues emerging from the rise of digital media, e.g. privacy, security and surveillance, piracy and intellectual property, digital divide, etc. (see, e.g. Bynum 2008, van den Hoven \& Weckert 2008, Himma \& Tavani 2008, Ess 2009, Floridi 2010). From this list of topics, however, it is not too difficult to see digital media as mainly conceived by them as a source of (new) moral problems to be dealt with. As a result, the focus of ICE has mostly been on responding to these new moral problems, which are often expressed in terms of what is 'right' or 'just'; and, questions about the relations between digital media and the good life are often left in the background. ${ }^{2}$ In other

1 For an overview of ICE and its history, see Bynum (2008).

2 To be fair, discussion on technology and the good life is not entirely absent. Notably, Borgmann $(1984,1999)$ has examined the impacts of technology, e.g. digital media, on the life of contemporary people, and he has offered a critique of technology through 
words, what is often missing in ICE is an explicit discussion of the relations between digital media and the good life, especially in a more balanced and constructive manner.

In this respect, there is another approach to the study of digital media which deserves special mentioning. Geert Lovink and his collaborators have proposed "Net criticism" as an approach for studying the impacts of digital media on people's lives (see, e.g. Lovink 2002, 2008, 2011/2003). ${ }^{3}$ As Lovink notes, "Net criticism is a call for critical intellectual engagement" (Lovink 2011/2003, p. 9), which aims to produce an informed (public) discourse that can transcend the uninformed and hastily accepted utopian and dystopian visions of digital media through critical (self-)reflection. In order to do so, however, Lovink argues that critics cannot examine digital media and its impacts only from a distance, but they must respond to and interact with existing views on digital media and its impacts. Net criticism, therefore, is both more balanced and constructive than most research in ICE. It is more balanced, because it does not start with the assumption that digital media is primarily a source of moral problems. And, it is constructive, because it strives to recommend specific ways to reform and transform digital media and digitallymediated practices that allow people to have better relations with digital media and enable them to live better lives with them (Lovink 2011, Introduction).

The present study aims to emulate Net criticism's balanced and constructive manner of studying the impacts of digital media, focusing on the relations between digital media and the good life. However, I want to move away from the concept of criticism in 'Net criticism' because of the derogative connotation of the term 'criticism'. ${ }^{4}$ In our attempts to produce an informed (public) discourse on digital

what he calls the "device paradigm" and "focal things and practices" (see also Higgs, Light \& Strong 2000). While Borgmann's works are important, however, his critique is too often one-sidedly negative (see, e.g. Verbeek 2002, 2006).

3 See the website of Institute of Network Culture (http://networkcultures.org). The Institute is founded and directed by Lovink. Since its establishment in 2004, the Institute has organised a variety of (public) events and has published a number of (scholarly and popular) publications.

4 In fact, Lovink does not use 'criticism' in a derogative manner (see Lovink 2011, pp. 11-14), and he thinks that the term 'criticism' is suitable for his purpose. While I agree that the term 'criticism' is not necessarily derogative, I think the objective of Net criticism, i.e. to produce an informed (public) discourse that can transcend the uninformed and hastily accepted utopian and dystopian visions of digital media through critical (self-)reflection, can be better realised by clearly dissociating itself from any potential derogative connotations of the term 'criticism'. 
media, we inevitably have to reflect on both the positive and negative aspects of digital media. Net criticism, I think, can serve us better if it can shed away the derogative connotation of 'criticism'. More importantly, when critics of digital media offer their criticisms, they are simultaneously recommending specific ways to reform and/or transform digital media. Hence, I think it is more fitting to speak of 'Net recommendation' rather than 'Net criticism', and thus I shall use 'Net recommendation' as the title of my study. ${ }^{5}$

In the remainder of the Introduction, I briefly discuss why the good life is often omitted in normative analysis. I argue that although there is, indeed, philosophical discussion of the good life, it falls short of answering the question of 'how should I live?', because it mainly focuses on analysing the concept of the good life. I then discuss why social, cultural and historical circumstances ${ }^{6}$ have to be incorporated into our normative analysis if we are to answer this question properly. Finally, I end the Introduction with an overview of the present study.

Before proceeding, it should be noted that I use the term 'digital media' as synonymous with similar terms such as 'new media' and 'information and communication technologies' (ICTs) throughout this study. I use 'digital media' instead of 'new media' or 'ICTs' because, firstly, I want to avoid the temporal and ideological connotation of 'new is always better' implied by the word 'new' in 'new media' (Lister et al., p. 11); and, secondly, I believe 'ICTs' is too broad as a term for the present study, as it can also be used to refer to pre-Internet analogue information and communication technologies, e.g. postal system, telephony, etc. 'Digital media', on the other hand, does not have the temporal and ideological connotation of 'new media', and it appears to best capture what is unique about the technologies and technologically-mediated practices I discuss in this study.

5 I use the term 'recommendation' also because, I think, it nicely captures an idea central to the present study, namely the questions related to the good life are often about decisions and actions that is neither obliged (i.e. what a person must do) nor impermissible (i.e. what a person must not do). In this respect, I think the term 'recommendation' helps to highlight the importance of the normative judgements on the things a person ought to do beyond what he is obliged to, as well as the things a person ought not to do even if they are not impermissible to do so.

6 Depends on the scope of normative analysis, personal circumstances should also be incorporated as well. Since the present study focuses primarily on the discussion on digital media and digitally-mediated practices at a public level, I shall omit personal circumstances here. 


\section{Ethics, the Good Life and Digital Media}

Charles Taylor states that "moral philosophy has tended to focus on what is right to do rather than on what is good to be, on defining the content of obligation rather than the nature of the good life", and he laments that moral philosophy has thus become "cramped and truncated" (Taylor 1989, p. 3). In a similar vein, Axel Honneth notes that " $[i] n$ the last three decades, [normative political philosophy] has essentially limited itself to evaluating the normative order of societies according to whether they fulfil certain principles of justice. Despite the success of this approach [...], this approach has lost sight of the fact that a society can demonstrate a moral deficit without violating generally valid principles of justice" (Honneth 2008, p. 84). Honneth calls those questions left out by normative political philosophy "ethical questions", which are about what is "desirable beyond all consideration of what is just" (Honneth 2008, p. 84). Taylor and Honneth have insightfully pointed out that there are some ethical questions that go beyond what is right and what is just, but have mostly been omitted in moral philosophy and normative political philosophy. I think those questions are precisely the questions pertaining to the good life, and they can be summarised by the question: how should I live?

However, if the questions pertaining to the good life are as important as Taylor and Honneth have suggested, why are they being omitted in moral philosophy and normative political philosophy? I believe two ideas widely held in moral philosophy and normative political philosophy are responsible for the omission, and they are (i) the idea of reasonable pluralism, and (ii) the idea of the person as an autonomous, rational being.

The idea of reasonable pluralism is best illustrated by John Rawls, who points out that we are now living in a world characterised by "the fact of reasonable pluralism," i.e. "a pluralism of comprehensive religious, philosophical, and moral doctrines [and, more importantly,] a pluralism of incompatible yet reasonable comprehensive doctrines" (Rawls 1993, p. xvi). And, here, the term 'comprehensive doctrines' refers to "conceptions of what is of value in human life, and ideals of personal character, as well as ideals of friendship and of familial and associational relationships, and much else that is to inform our conduct, and in the limit to our

\footnotetext{
7 The question of 'how should I live?' is often used in contrasting the right (i.e. morality in a narrow sense) with the good (i.e. morality in the broad sense), see Williams (1985). It is less often used in contrasting the just with the good because the question is formulated personally with ' $I$ ' (or ' $w e^{\prime}$ ), but the formulation of the question itself should not prevent us from seeing the differences between the just and the good.
} 
life as a whole" (Rawls 1993, p. 13). In Rawls's view, therefore, given "the fact of reasonable pluralism", the best hope of normative political philosophy is to achieve an "overlapping consensus" (Rawls 1993, p. 133-172). Since reasonable comprehensive doctrines can be incompatible but yet reasonable, they have to be set aside in political deliberation. Similarly, because any answers to the question of 'how should I live?' are based on people's comprehensive doctrines, the question does not, and cannot, have a legitimate place in the realm of politics, too. So construed, a sharp separation between the just and the good can be discerned in the idea of (Rawlsian) reasonable pluralism. Hence, this idea naturally leads to an exclusion of the questions pertaining to the good life, as they are not legitimate questions in normative political philosophy. ${ }^{8}$

Another idea responsible for the omission of the good life in normative analysis is the idea of the person as an autonomous, rational being. According to this idea, persons are self-determining and self-responsible for their lives, and they are the only ones who have authority over their choices and actions. This idea also entails that individuals can pursue their life projects via any means they conceive to be most suitable and efficient to them, provided that those means are not morally dubious, i.e. they are not morally wrong or unjust. So construed, such an idea of the person affirms individuals themselves to be the only and the best persons in deciding and acting on those matters pertaining to their (good) lives. Accordingly, the good life is an entirely subjective matter. Hence, no interference on people's choices and actions pertaining to their (good) lives can be justified, and thus the good life is beyond the reach of moral philosophy.

Yet, digital media clearly has transformative impacts on our lives. Ignoring the question of 'how should I live?' will unnecessarily narrow the scope of normative analysis of digital media. In later chapters, I shall return to these ideas and show they do not provide sufficient reasons for omitting the question from normative analysis. ${ }^{9}$ For now, I think it suffices to say that broadening the scope of normative analysis of digital media to include the question is desirable, as it allows our normative analysis to be more comprehensive.

\footnotetext{
8 A similar story can be told with the right and the good as well, i.e. since our answers to the question of 'how should I live?' is based on our comprehensive doctrines, which can be incompatible and yet reasonable, the best hope of moral philosophy is to achieve an "overlapping consensus" too. Accordingly, the questions pertaining to the good life are also not legitimate questions in moral philosophy.

9 See, esp. Chapter 1 and Chapter 6.
} 
It is perhaps misleading to claim that research on the good life (or well-being) is entirely absent in philosophical discussion. ${ }^{10}$ There is, indeed, much discussion of what the concept of the good life amounts to. However, we have to distinguish carefully the descriptive project from the normative project in constructing a theory of the good life. The goal of the descriptive project is to provide a formal analysis of the (semantic) meaning of 'the good life', and it is distinct from the normative project, which attempts to provide answers to the question of 'how should I live?'. The descriptive project, as Valerie Tiberius notes, seeks "to give an analysis of the nature of well-being [by articulating] the criterion (or criteria) that any thing must meet in order to count as a source or cause of [the good life]" (Tiberius 2004, pp. 295-296). In other words, it aims to analyse the concept of the good life (or to offer a definition of it), but it does not aim to answer the question of 'how should I live?'.

There are three most notable families of theories of the good life for the descriptive project, and they are hedonism, desire theories and objective list theories. In its simplest formulation, hedonistic theories state that pleasure is the only criterion of the good life. Simple desire theories state that satisfaction of desires is the only factor contributing to the good life. Finally, simple objective list theories state that there is a list of goods comprising the good life regardless of

10 Throughout the study, I use the term 'the good life' and 'well-being' (and other similar terms, e.g. 'quality of life', etc.) interchangeably. My use of 'the good life' and 'well-being' as synonymous is not uncontroversial, see, e.g. Haybron (2008, esp. Chapter 2). However, I am inclined to agree with Velleman (2000) that well-being is not additive, and that a person's (good) life must be evaluated as a whole because temporality and sequentiality of a person's life events necessarily influence his life. Consider Velleman's example,

"[There are] two different lives that you might live. One life begins in the depths but takes an upward trend: a childhood of deprivation, a troubled youth, struggles and setbacks in early adulthood, followed finally by success and satisfaction in middle age and a peaceful retirement. Another life begins at the heights but slides downhill: a blissful childhood and youth, precocious triumphs and rewards in early adulthood, followed by a midlife strewn with disasters that lead to misery in old age. Surely, we can imagine two such lives as containing equal sums of momentary well-being $<$ Yet even if we were to map each moment in one life onto a moment of equal well-being in the other, we would not have shown these lives to be equally good." (Velleman 2000, p.58)

If Velleman is right, then well-being (or the good life) should not be evaluated in isolation of the temporality and sequentiality of a person's life events. Accordingly, there should not be a sharp difference between well-being and the good life, as both terms should be used to refer to a person's well-being in his overall lifespan, i.e. his good life. 
whether people approve the list or not. Hedonism, desire theories and objective list theories are all criticised and defended thoroughly. However, as my aim is not to criticise or defend any of these theories, I shall not recite the debate here. It is sufficient to note that the debate is still on-going, and there is no general consensus as to which theory gives the best analysis of the concept of the good life.

What is relevant for here is that all theories of the good life in the descriptive project, as it is characterised by Tiberius, have the tendency to conceptualise 'the good life' as a universal concept. For example, (simple) hedonistic theories conceptualise the good life exhaustively in terms of acquisition of pleasure and avoidance of pain, and they take this account to be true for every person across different social, cultural and historical circumstances. The same is true for (simple) desire theories, too. Desire theories conceptualise the good life to be invariably about satisfaction of desires. Finally, (simple) objective list theories attempt to specify a list of goods that are necessary and sufficient for the good life of anyone from anywhere. This tendency has led philosophers to conceptualise the good life in a minimal and context-independent manner. Disconnected from the social, cultural and historical circumstances, the concept of the good life in the descriptive project is bound to be abstract and theoretical. Consequently, if such a concept is also being used in the normative project, the answer(s) to the question of 'how should I live?' will be minimal and context-independent as well.

However, if the normative project in the theory of the good life aims to provide answers to the question of 'how should I live?' (and, in the present study, this question can be roughly reformulated as 'how should I live with digital media?'), then minimal and context-independent concepts of the good life will always be inadequate, because they are devoid of the circumstantial factors necessary for offering practical recommendations. ${ }^{11}$ The inadequacy of minimal and context-independent concepts of the good life as such is nicely captured by Bernard Willams's complaint about contemporary moral philosophy, where he points out that "it is too abstract and theoretical to provide any substance to ethical life [...], and it is precisely the use of "thick" ethical concepts, among other things, that contributes to a more substantive type of personal ethical experience than theory is likely to produce" (Williams 2005, p. 48). The lesson from Williams's complaint is that any normative theories containing only minimal and context-independent normative concepts cannot be of practical relevance, i.e. they cannot be action-

11 I provide a more detailed argument for this claim in Chapter 1, section 1.6. 
guiding. Still, it does not follow from this that the entire enterprise of descriptivecum-normative theories (of the good life) is a failure. I think the lesson to be learned from Williams's complaint is that any theories of the good life for the normative project, namely if they are to answer the question of 'how should I live?', must go beyond a minimal and context-independent concept of the good life and incorporate circumstantial factors into their answering of the question. ${ }^{12}$

At the same time, if we take seriously the need to incorporate circumstantial factors into normative analysis of digital media and the good life, it also means that different cultures and societies need to have their own normative analyses too. This is because different cultures and societies give rise to their own circumstantial factors. Accordingly, intercultural (and cross-cultural) normative analysis of digital media and the good life is both important and fruitful. It is important because only then can we have a more comprehensive account of how digital media does, and can, influence our lives; and, it is fruitful because, by looking at other cultures and societies, intercultural normative analysis of digital media and the good life can provide us different perspectives for offering practical recommendations that are otherwise unavailable.

\section{An Overview of the Dissertation}

The main goal of the present study is to (re)assert the importance of actual discourses in our normative analysis of the relations between digital media and the good life. I illustrate the way(s) in which we can incorporate actual discourses into normative analysis of digital media and the good life, as well as the role(s) they can play in our normative judgements on digital media and digitally-mediated practices. More specifically, I offer a novel approach to normative analysis of digital media (and the good life) that takes seriously (and, ideally, also interacts with) actual discourses, and apply it to popular discourse on digital media and the good life. This approach, as I shall argue, allows us to have a better understanding of our normative

\footnotetext{
12 It should be noted hedonism, desire theories and objective list theories are normative-even in the descriptive project-by stating what the good life is (or, more accurately, what the good life should be) and thus affirming a normative standard for the good life. Hence, my point is not that the three families of theories of the good life cannot be normative. Instead, my point is that an emphasis on the descriptive project may lead to a particular way to engage in the normative project, i.e. using the concept of the good life in the descriptive project in the normative project, which, as Williams has complained, is insufficient for guiding actions.
} 
judgements on the impacts of digital media has (or will have) on the good life and, at the same time, enables us to answer the question of 'how should we live with digital media?' more adequately.

Yet, the approach I propose in the present study is not only useful to normative analysis of digital media and the good life in an intra-cultural context, i.e. the critical study of the impacts of digital media on the good life within a culture, it is also useful in the intercultural contexts. By applying this approach to Internet policy documents and a set of opinion pieces in a major newspaper in China, I show that each culture should be examined in its own right. In other words, I try to apply the approach at a local level, as well as a global level. In this way, I hope the present study will add to the growing body of research in intercultural (and cross-cultural) studies of digital media.

At the most general level, I hope the present study will contribute to the field of ICE and critical studies of digital/new media by (re)turning to the good life through an analysis of the relations between digital media and the good life.

In Chapter One, I propose and elaborate my approach to normative analysis of digital media and the good life, which is based on Michael Walzer's idea of social criticism. This approach is characterised by five features, i.e. (i) hermeneutical, (ii) immanent, (iii) participatory, (iv) empirical and (v) pluralistic. It takes seriously the actual discourses (e.g. popular discourse) on digital media and urges researchers to integrate them into their normative analysis. Since the focus of my study is on the relations between digital media and the good life, I identify a specific type of actual discourses on digital media that is relevant to the present study, i.e. prudential appraisals of digital media. Here, I argue that prudential appraisals of digital media are normative, and that their normativity is grounded in our practical identity (or our self-interpretation and self-understanding). I discuss how, and in what sense, practical identity is the source of normativity, and point out what this means to normative analysis of digital media and the good life. Finally, I end the chapter by offering an additional argument for the indispensability of prudential appraisals of digital media in normative analysis of digital media and the good life.

As I argue in Chapter One, prudential appraisals of digital media are normative and their normativity comes from people's self-interpretation and selfunderstanding. In order to properly understand our normative judgements on the impacts of digital media on the good life, it is necessary first to explicate the normative ground(s) behind the judgements, i.e. our mode(s) of self-interpretation and self-understanding. In Chapter Two, through a discussion of the works of Charles Taylor, Anthony Giddens and Ulrich Beck, I identify three different 
notions of the self in modern and late modern societies that serve this purpose, i.e. the disengaged self, the expressive self and the reflexive self; and, at the same time, I also identify the ideals (or the views of the good life) these notions of the self embody. Finally, I support my account of the views of the good life in modern and late modern societies by looking at the empirical research conducted by Ronald Inglehart and his colleagues. The main objective of this chapter, in short, is to lay the ground for my analysis of prudential appraisals of digital media in the next chapter by recollecting the normative and evaluative resources for (re)interpreting and understanding them.

In Chapter Three, I apply the Walzerian approach with the notions of the self I have discussed in Chapter Two to prudential appraisals of digital media. I examine two sets of prudential appraisals of digital media: the first set focuses on the impacts of digital media on culture and society, which influence people's (good) lives by transforming the exteriors of their lives; and, the second set focuses on the impacts of digital media on our brain, mind and/or the self, and how it influences people's (good) lives by transforming their interior lives. More specifically, I attempt to show that prudential appraisals of digital media-and, for that matter, any normative judgements on the impacts of digital media on the good life-are best understood with the notion(s) of the self. This, I also attempt to show, has an important implication to normative analysis of digital media and the good life, namely we should (re)direct our attention to the question of 'who we should be in a digitally-mediated world?'

Chapter Four is devoted to explore a different notion of the self, i.e. the Confucian self in (contemporary) China. I argue that the modern self and the late modern self I discuss in Chapter Two are inadequate for a comprehensive normative analysis of digital media and the good life, because they are not readily applicable to societies that have a different cultural root, and thus a different trajectory of modernisation. I propose that we should move beyond the idea of singular modernity and replace it with the idea of plural modernities, which allows us to properly acknowledge the importance of various cultural roots. With the idea of plural modernities in place, I explore the Confucian self and the view of the good life it embodies. Similar to what I have done in Chapter Two, the aim of this chapter is to lay the ground for my analysis of prudential appraisals of digital media in (contemporary) China by exploring the normative and evaluative resources available in the Confucian tradition.

I have applied the Walzerian approach in an intra-cultural context in Chapter Three, but the approach is also useful in an intercultural context. In Chapter Five, I 
illustrate how the Walzerian approach can be applied at a global level. I analyse the Chinese Communist Party's Internet policy documents and a set of opinion pieces published by a major Chinese newspaper, Guangming Daily (光明日報) with the Confucian self, and illustrate the fundamental role of Confucian values (and the Confucian view of the good life) in grounding China's Internet policy and normative judgements in the opinion pieces. I argue that if it is indeed true that China's Internet is informed by a different normative and axiological foundation, i.e. the Confucian self, then the question of 'whether the Internet is good or bad?', or the question of 'how we should live with digital media?', should also be answered differently-in a socially and culturally sensitive manner. To illustrate this, I offer a discussion of social media from the Confucian perspective. I show that there is a prima facie incompatibility between the Confucian way of (good) life and social media, but I also point out that the incompatibility between them may be resolved. In short, the lesson of this chapter is that different societies require their own normative analysis of digital media with their own normative and evaluative resources.

Implicit in the project of Net recommendation and the Walzerian approach to digital media and the good life is philosophers' responsibility to proactively offer practical recommendations to the public, i.e. users of digital media. However, the practice of recommendation is often faulted as paternalistic, and thus is considered to be morally undesirable. This criticism must be answered if the project of Net recommendation or the Walzerian approach is to be considered as a (morally) feasible option. In Chapter Six, I look at this criticism more closely. I argue that offering recommendations is indeed paternalistic, but we should not see it as morally problematic, because paternalism is inevitable in our technologicallymediated lives. Hence, philosophers should not be shied away from the practice of recommendation-especially only if it is for the worry over paternalism. Although there are philosophers who already take seriously the inevitability of paternalism in our technologically-mediated lives via the idea of design ethics, I point out that it must too be supplemented by the practice of recommendation. And, I illustrate how the Walzerian approach may supplement design ethics, as well as how it can actually minimise the worry over paternalism from the practice of recommendation.

Finally, in the Epilogue, I offer a brief summary of this study and discuss several practical implications of the project of Net recommendation and the Walzerian approach. 



\section{ChAPTER ONE}

\section{Appraising Digital Media and the Good Life}

A Walzerian Approach

New technological developments bring with them both hopes and fears. The development of digital media is no exception. Scholars and critics have responded to the changes brought about by digital media with extensive discussion regarding their ethical, social, cultural, political, economic and legal implications. While many of these issues have already been taken up by researchers, systematic normative analysis into the impacts of digital media on people's good lives is relatively rare. An analysis as such will study the relations between digital media and the good life and, at the same time, critically examine them in order to determine which relations between human beings (or society) and digital media will allow them to flourish. The overall aim of this chapter is to present an approach to digital media and the good life, and thereby to contribute to the normative analysis of digital media.

From a philosophical perspective, two distinct approaches are available with regards to analysing the relations. Philosophers have long sought to answer questions pertaining to the good life, and major theories of the good life are well established in philosophy. Hence, one approach is to build on one's favourite philosophical theory of the good life by elaborating upon and defending it, and to evaluate the impacts of digital media on people's good lives based on the view of the good life specified by such a theory. This can be termed the theory-driven approach. Another approach starts not with (philosophical) theories, but rather with actual 
appraisals of digital media. Supporters and detractors of digital media have pronounced, on various occasions, the manner in which digital media may contribute to or detract from the good life. These appraisals are, in effect, recommending specific human-digital media relations (or society-digital media relations). In doing so, they are also reflecting the critics' views of the good life. By uncovering the values and assumptions underlying these appraisals, researchers can connect digital media to different views of the good life expressed by them. This interpretative approach to normative analysis then allows researchers to use the disclosed views of the good life to evaluate the impacts of digital media on people's good lives and to offer practical recommendations. ${ }^{1}$

In Section 1.1, I develop and elaborate an approach to normative analysis of digital media and the good life which resembles the second approach (i.e. the interpretative approach), and I show that this approach is a viable alternative to the first approach (i.e. the theory-driven approach). Yet, what are the differences between a theory-driven approach and an interpretative approach? Drawing from Michael Walzer's discussion of the idea of social criticism, I explain their differences. Furthermore, building on Walzer's idea of social criticism, I introduce a Walzerian approach for the present study. Since the approach I favour is based on actual appraisals of digital media, I identify the type of appraisals that is central to the present study. In Section 1.2, I begin by describing what an appraisal is, and then distinguish a type of appraisals, i.e. prudential appraisals of digital media, from other types of appraisals. I analyse the term 'good for' and the structure of prudential appraisals of digital media in Section 1.3. Such an analysis is important, because it provides a framework for interpreting and understanding prudential appraisals of digital media. And, since whether an interpretative approach is appropriate for the present study is contingent on the nature of the views of the

\footnotetext{
1 The notion of interpretative approach is not unambiguous. There are numerous ways to conceptualise an interpretative approach, e.g. Yanow \& Schwartz-Shea (2006). But, as Yanow and Schwartz-Shea have pointed out, although there are differences in methods and focuses in various interpretative approaches, they all emphasise the importance of language and the practice of sense-making as opposed to the positivistic standards prevailing in natural sciences. Moreover, interpretative approaches also seek to transcend the fact-value distinction that is inherent to the positivist picture of social sciences (Yanow \& Schwartz-Shea 2006, pp. xi-xiii). The Walzerian approach I propose in this chapter shares with other interpretative approaches the emphasis on language and sense-making, and it also attempts to transcend the fact-value distinction by taking seriously existing views of the good life as the sources of normativity.
} 
good life in prudential appraisals of digital media, I examine the nature of the views of the good life in prudential appraisals of digital media, and show that the sociocultural nature of the views of the good life in prudential appraisals of digital media supports my Walzerian approach in Section 1.4. Finally, I end this chapter with an additional argument for the Walzerian approach on the basis of the indispensability of prudential appraisals in normative analysis of digital media and the good life.

\subsection{A Walzerian Approach to Digital media and the Good Life}

I have briefly outlined two approaches to normative analysis of the good life, i.e. the theory-driven approach and the interpretative approach, which mirror two forms of social criticism described by Michael Walzer as disconnected criticism and connected criticism. In this respect, a survey of Walzer's discussion of the idea of social criticism will help to illuminate the differences between the two approaches. It will also, I hope, highlight the strengths of the interpretative approach. Although Walzer's idea of social criticism is not uncontroversial, it is beyond the scope of the present study to offer a thorough defence of it. Instead, my modest objective is only to attempt to articulate a version of this approach for normative analysis of digital media and the good life. My objective, in other words, is to make the Walzerian approach an option worth considering.

\subsubsection{Walzer and the Two Forms of Social Criticism}

In various places, particularly in Interpretation and Social Criticism ${ }^{2}$ (1985), The Company of Critics (1988) and Thick and Thin (1994), Walzer discussed the idea of social criticism. ${ }^{3}$ For Walzer,

"[s]ocial criticism is a social activity. "Social" has a pronominal and reflexive function [...] which names subject and object at the same time. No doubt, societies do not criticise themselves; social critics are

2 Walzer first delivered the Tanner Lectures on Human Values entitled "Interpretation and Social Criticism" in 1985. The lecture manuscript was subsequently published by Harvard University Press in 1987 with the same title. In this chapter, the page numbers refer to the page numbers on the manuscript.

3 Walzer's discussion of the idea of social criticism is scattered in different parts of his articles and books. In this chapter, I focus mainly on the idea elaborated and defended in Interpretation and Social Criticism because it provides the most explicit theoretical statement of the idea. See also, Walzer (1981, 1984, 1989, 1993). 
individuals, but they are also, most of the time, members, speaking in public to other members who join in the speaking and whose speech constitutes a collective reflection upon the conditions of collective life." (Walzer 1985, p. 30)

Through this definition, Walzer has forged an inseparable link between critics and the activity of social criticism. He distinguishes two forms of social criticism, i.e. disconnected criticism and connected criticism, and locates their fundamental differences in the stance of critics and their relationship to the community to which they belong. It is precisely the stance taken by the connected critics and their relationship to their own community, in Walzer's view, that make connected criticism a superior form of social criticism. To see why connected criticism and, analogously, the interpretative approach, are preferable, I shall start with Walzer's account of disconnected critics and connected critics.

\subsubsection{Disconnected Critics versus Connected Critics}

Walzer points out that disconnected critics and connected critics are driven by two different notions of the ideal critic (and its preliminary requirements). In Walzer's description, disconnected critics are those who

"stand outside the common circumstances of collective life. [For the disconnected critics, c]riticism is an external activity; what makes it possible is radical detachment [...] First, critics must be emotionally detached, wrenched loose from the intimacy and warmth of membership: disinterested and dispassionate. Second, critics must be intellectually detached, wrenched loose from the parochial understandings of their own society (standardly taken to be selfcongratulatory): open-minded and objective." (Walzer 1985, p. 3I)

Since personal and intellectual detachment is a prerequisite for disconnected criticism, a disconnected critic must always (attempt to) be "an outsider, a spectator, a 'total stranger', a man from Mars" (Walzer 1985, p. 33). Disconnected critics, in other words, must break free from the particulars of their society, culture and history. Particulars are not, and should not be, relevant to social criticism because disconnected critics must judge, as Walzer (borrowing Thomas Nagel's term) suggests, from "no particular point of view" (Walzer 1985, p. 6).

On the contrary, connected critics do not prize personal and intellectual detachment. Instead, they identify themselves as members of their own community, and engage with those who share the same community. Connected critics are not 
guided by either impartiality or universality, which is achieved by adopting Nagel's "no particular point of view". Rather, they take seriously the particulars of their own society, culture and history, and apply "standards that [they] share with the others to the others, [their] fellow citizens, friends and enemies" (Walzer 1985, p. 43). ${ }^{4}$ Hence, connected criticism always presumes a view from somewhere or, more accurately, a view from where the connected critics belong to. To recapitulate, a connected critic in Walzer's terms is

"the local judge [...], who earns his authority, or fails to do so, by arguing with his fellows-who, angrily and insistently, sometimes at considerable personal risk (he can be a hero too), objects, protests, and remonstrates. This critic is one of us. Perhaps he has travelled and studied abroad, but his appeal is to local or localised principles; if he has picked up new ideas on his travels, he tries to connect them to the local culture, building on his own intimate knowledge; he is not intellectually detached. Nor is he emotionally detached. Social criticism, for such people, is an internal argument." (Walzer 1985, pp. 33-34)

The different stances taken by the two kinds of critics, and the different relationships they have with their community, can be traced back to their views of morality. In Interpretation and Social Criticism, Walzer has identified three paths in moral philosophy, i.e. "the path of discovery", "the path of invention" and "the path of interpretation" (Walzer 1985, p. 4). Here, the first two paths readily lend support to disconnected criticism, while the last naturally leads to connected criticism.

Walzer claims that the path of discovery is akin to religious revelation. For this path, it is presumed that there is a creator of morality, e.g. God, and only "with his help and with the help of his servants" do people come to discover moral norms and

4 Here, the phrase 'taking seriously the particulars' can be interpreted in a weak sense and a strong sense. Interpreted in a weak sense, the particulars are considered to be (instrumentally) useful for critics to arrive at specific normative conclusions; and, interpreted in the strong sense, the particulars are considered to be constitutive of the normative conclusions; or, in other words, normativity is derived from the particulars. In this study, I am using the phrase in the strong sense, I will return to this issue in section 1.6. 
principles, and integrate them into their moral life (Walzer 1985, p. 5). ${ }^{5}$ With regards to the path of invention, people are to "design the moral world" because "there is no pre-existent design, no divine or natural blueprint to guide us" (Walzer 1985, p. 10). The challenge for designing a moral world, of course, is that the designer(s) "must somehow be authorised to speak for all of [the people] or, alternatively, all of [them] must be present and accounted for from the beginning" (Walzer 1985, p. 11). Walzer cites John Rawls's veil of ignorance and Jürgen Habermas's ideal speech situation as answers to this challenge. In one way or another, Rawls and Habermas have answered the challenge by eliminating the particulars from their designs. In other words, they have attempted to invent a moral world that can be inhabited by anyone who satisfies a minimal level of rationality. To maximise inclusion, the representation of and, for that matter, the presentation of persons can only be minimal in the process of design. This is necessarily so because the invented morality and, relatedly, the set of invented moral norms and principles, gain force only if "we have participated, or can imagine ourselves having participated, in its invention" (Walzer 1985, p. 12).

Finally, the path of interpretation focuses on "ourselves, our own principles and values" (Walzer 1985, p. 17). It proceeds by (re)interpreting the existing morality of one's own community. We are, as Walzer notes, always in "someplace of value" (Walzer 1985, p. 16). Accordingly, both discovery and invention are superfluous, if not pretentious, as critics and (moral) philosophers already have what they are supposed to discover or invent, that is - the existing morality of their community (Walzer 1985, pp. 18-19). Moreover, the existing morality is already normative, as our moral languages are derived from that morality; or, in Walzer's terms, the existing morality "provides us with everything we need to live a moral life-including the capacity for reflection and criticism" (Walzer 1985, p. 20).

It is not difficult to see how the path of discovery and the path of invention lend support to disconnected criticism. Both of them locate morality, i.e. the normative standards, outside of one's own community. Morality, when being seen as a discovery or invention, is independent of and separable from the society, culture and history. For the discoverers, morality is created by God or God-like entities (or, in secular morality, it is the Truth in science, philosophy, etc.). Critics

\footnotetext{
5 The path of discovery does not preclude secular morality. Walzer clearly acknowledges the possibility of natural revelation, and he uses utilitarianism as an example of non-religious discovery (Walzer 1985, pp. 6-8).
} 
who follow this path command from a privileged perspective, and they are not ultimately responsible to the people of their community, but rather to God and its messages (or the Truth). For the inventors, their creations are intended to be universal, applicable across time and space. Hence, the specific ties they and others have with their own community are nothing but obstacles in the process of design. The invented morality, without referring to a particular society, culture or history, offers critics something seemingly universal, and sways them away from the existing morality.

Meanwhile, it is also not difficult to see how the path of interpretation leads to connected criticism. This path requires critics to focus on and employ what they already have, i.e. the existing morality. In other words, what critics have to do is to (re)interpret that morality. In this sense, connected criticism is itself a proper instance of interpretative (moral) philosophy, and connected critics are effectively (moral) philosophers in Walzer's account.

\subsubsection{Connected Criticism and Its Viability}

Walzer notices that the notions of connected critics and connected criticism are deemed by some to be philosophically untenable. To revive the viability of connected critics and connected criticism, he offers two sets of arguments to his commentators. I shall call the first set of arguments the inevitability of interpretation, in which he tries to show that "philosophical discovery and invention [...] are disguised interpretations; there is really only one path in moral philosophy" (Walzer 1985, p. 20). His conclusion as such, however, has to be qualified. Walzer does not deny the possibility of discovery or invention in moral philosophy; what he denies is the idea that it is possible to do without interpretation. Accordingly, Walzer's claim is that for the discovered morality and the invented morality, if they are to dwell on the ordinary moral discourse, they too must be interpreted. His claim, therefore, is about the applicability and continuity of morality, and should not be mistaken for a claim about the formation of morality. In short, Walzer's argument from the inevitability of interpretation is that if moral philosophising necessarily involves interpretation, then connected criticism, being essentially an interpretative enterprise, should not be viewed as peculiar or inferior to other methods in moral philosophy.

The second set of arguments is based on the presumed function of social criticism or morality: that is, if social criticism is to critically reflect on individual behaviours and social practices and to bring changes to them; or, similarly, if morality is to guide us through our moral world and to tell us what is good and 
what is right, then connected criticism or (re)interpretation of existing morality will be a more effective and moral option than disconnected criticism.

Walzer argues that "[o]ur categories, relationships, commitments, aspirations are all shaped by, expressed in terms of, the existing morality" (Walzer 1985, p. 20). ${ }^{6}$ If Walzer is right, then our moral conducts will always be governed by a morality that we have already internalised. Connected criticism, therefore, is strategically more effective than disconnected criticism, as connected critics appeal to local norms and values, which are already accepted by those who are living in the critics' community. An ideal connected critic, after all, as Walzer claims, will "[speak] in the first person plural. This is what we value and want, he says, and don't yet have. This is how we meant to live and don't yet live" (Walzer 1988, p. 230). Unlike disconnected criticism, connected criticism is neither new to nor alienated from the people. Hence, it is more effective in bringing changes to individual behaviours and social practices, as people are already motivated internally by what they have already accepted. ${ }^{7}$

The argument from the effectiveness of connected criticism also has a moral dimension. Walzer argues that connected criticism help prevent critics from falling into two moral pitfalls easily committed by disconnected critics. The first moral pitfall is directly related to the function of social criticism and its effectiveness. As Walzer points out, disconnected criticism, "derive[d] from newly discovered and invented moral standards, [...] presses its practitioners toward manipulation and compulsion... [I]nsofar as the critic wants to be effective [...], he will find himself driven to one or another version of an unattractive politics" (Walzer 1985, p. 55). When critics try to persuade their people there are other and, more importantly, better options available to them; by the fact of its disconnectedness, disconnected criticism will always remain as something superimposed on people and their community. Persuasion by disconnected critics, then, easily becomes coercion. Yet, if coercion is morally dubious, following Walzer's argument, we should also avoid disconnected criticism as well.

The second moral pitfall arises from the purported decisiveness and universality of disconnected criticism. The attractiveness of disconnected criticism,

\footnotetext{
6 It is clear that Walzer's claim is based on the relatively uncontroversial assumption that human beings are sociocultural beings. But, what is less clear is how human beings' sociocultural nature is related to their judgements. I shall return to this in section 1.5.

7 Similarly, Moody-Adams has argued that assimilation or dissemination of morality via entirely new or alienated moral concepts is implausible. See, Moody-Adams (1999).
} 
as Walzer suggests, comes from the hope that we can end a moral debate "once and for all" (Walzer 1985, p. 43). Disconnected criticism is presumably messages from God (or the Truth) or an agreement that is determined by a process of design which has involved every person (either actually or hypothetically); by invoking a single truth or an agreement of all, disconnected criticism is portrayed as decisive. In other words, it appears to provide final(ised) verdicts without the need for further refinement. It is precisely such an appearance, enabled by its purported universality, troubles Walzer. He points out that the larger the scope of agreement there is, the smaller the range of choices that will remain to the people, as the issues that are agreed upon are considered to be final(ised) and settled. ${ }^{8}$ Accordingly, new comers "will not have much to say than 'That sounds right,' or 'I can think of no objections,' or 'I entirely agree"' (Walzer 1989, p. 28). As such, it leaves no room for other opinions or further discussion. What really troubles Walzer here, I think, is that agreements in philosophical discussion are artificial at best and distorted at worst. Sustained agreements can only be found in philosophers' idealised world; and, actual agreements are only "temporary stopping points" in a chain of continuous arguments. Disconnected criticism, therefore, does not only suppress differences and discussion; it also generates a deceptive appearance of stability, which, for better or worse, maintains the status quo.

Some, however, remain unconvinced by Walzer's arguments. In particular, they have faulted his notion of connected criticism for its anti-foundationalism and relativism. ${ }^{9}$ Walzer's commentators have argued that connected criticism will be groundless and unstable without being supported by a universal standard. Without a universal standard, they argue, even connected critics themselves cannot determine which interpretation (of existing morality) is better. Moreover, in Walzer's account, the correctness of interpretation appears to depend solely on the standards of the critics and their community. This worries his commentators, as the groundlessness and instability of connected criticism may then warrant immorality

8 For example, Walzer (1981) has illustrated this point by using the case of the human rights debate.

9 See, e.g. Barry (1990). Other than the charges of anti-foundationalism and relativism, Walzer's notion of connected criticism and his interpretative moral philosophy have also been subjected to the charge of conservatism. This charge, however, presupposes a static view of community, which Walzer has clearly rejected. For a defence against the charge of conservatism based on the idea of social and cultural exchange, see Sreenivasan (1998) and Moody-Adams (1999). 
and injustice. They argue that fights against immorality and injustice such as slavery, exploitation, murder, etc. require a solid grounding and universality, even if the moral norms and principles against immoral behaviours and unjust practices are cumbersome; or, that they have to be forced upon the people. In the light of immoral behaviours and unjust practices, they argue, neither effectiveness nor the moral pitfalls mentioned above are relevant.

I shall not repeat the debate between Walzer and his commentators here. Suffice it to say that Walzer will have no qualms regarding the need for a thin morality, i.e. a set of minimal, general codes of morality that enables criticisms of immoral behaviours and unjust practices. What Walzer disagrees with his commentators on is that the minimal morality should not precede the socially, culturally, and historically thick morality. In other words, thin morality should be hollowed out from the existing morality of different communities, but not the other way around (Walzer 1994). ${ }^{10}$

10 The debate on (moral) relativism deserves a lot more space than I can provide in the present study. But, it is useful to briefly illustrate how thin morality may figure in the Walzerian approach I propose in this chapter. Here, I contend the Walzerian approach to be (morally) relativistic in that what is good (or bad) is determined socially and culturally. However, as I have noted, Walzer will not deny the importance of thin morality for criticising immoral behaviours and unjust practices. Such a thin morality, I think, must be constructed out of the socially, culturally, and historically thick morality. In this sense, thin morality is not closed, universal or true a priori, it is constantly open to inputs from social, cultural and historical circumstances, sensitive to the particulars and only true a posteriori. Yet, being open, sensitive to particulars and contingent does not entail that no moral norms and principles can be shared in all cultures and societies. As Street (forthcoming) argues, moral relativism only requires moral norms and principles to be justified via different grounds. In other words, the Walzerian approach shall not deny the possibility of a set general moral norms and principles (see, section 1.1.2, pt. 5).

In effect, I suggest that the Walzerian approach should take a constructivist approach to (thin) morality, which takes the generally shared moral norms and principles as its basis. Accordingly, the Walzerian approach I propose here still allows us to (use thin morality to) criticise other behaviours and practices in other cultures and societies, provided that (i) our criticisms are based on moral norms and principles that are shared by them, or that (ii) we recognise and acknowledge our criticisms are based on normative standards of our own culture and society which may not apply to them. This, of course, leads to further questions concerning (a) the boundary of culture and society and (b) the effectiveness of (ii), which I cannot answer satisfactorily. I speculate that no sufficient answer can be provided to (a), because cultures and societies are not static but dynamic. However, precisely because of cultures and societies are dynamic, it is possible to expand the scope of moral norms and principles different cultures and societies (can) share. In 
Yet, the emphasis on immoral behaviours and unjust practices in the debate on the viability of connected criticism is especially instructive to the present study. So far, Walzer's notion of connected criticism has primarily been applied to moral philosophy and normative political philosophy; it has not received similar attention in research pertaining to the good life. This is important because the objections against connected criticism's anti-foundationalist and relativistic character in regards to moral philosophy and normative political theory are not immediately applicable to normative analysis of the good life. Normative questions on the good life typically have to do with "the best thing to do", whereas in moral philosophy and normative political theory the questions are centred on (moral) rightness and justice. Failures to do the best thing, however, do not make a person wrong (at least, not in the moral sense) or unjust. Hence, the need for obligation and prohibition is less pressing in normative analysis of the good life than in moral philosophy or normative political philosophy (Kupperman 1999, pp. 87-89). Now, normative analysis of digital media and the good life, in many cases, is also not about obligation and permissibility. Instead, it is, on many occasions, about amelioration, i.e. how to improve one's well-being by connecting oneself to (or disconnecting oneself from) digital media. As such, the emphasis on the good life should at least mitigate some worries concerning anti-foundationalism and relativism with respect to immoral behaviours and unjust practices.

If the aim of normative analysis of digital media and the good life is to effectively persuade people that some human-digital media relations (and societydigital media relations) are better than the others, then connected criticism, which motivates people from within, should be considered a viable, or even a comparably better, approach. More importantly, perhaps, is the fact that Walzer's disquiet with regards to the decisiveness and universality presupposed by disconnected criticism, as well as its tendency to shrink the space of deliberation, is not to be understated if Rawls is correct about "the fact of reasonable pluralism" (Rawls 1993, p. xvi). Connected criticism, which is an exercise of (re)interpretation of existing morality, is particularly fitting for normative analysis of the good life, as it has to be conducted in a socially, culturally and historically conscious manner. Hence, it constantly reminds us of the lingering "fact of reasonable pluralism". In light of the effectiveness of social criticism and the fact of reasonable pluralism, connected

this respect, a dynamic view of culture and society should relieve (ii), at least, to a certain extent. Also, see fn. 9 . 
criticism, and similarly an interpretative approach to digital media and the good life, appears to be a better option than its disconnected counterpart.

\subsubsection{A Walzerian Approach: A Proposal}

In the above discussion, I have summarised two forms of social criticism and the views of morality underlying them. I have also outlined some potential advantages connected criticism has over disconnected criticism when applied to normative analysis of digital media and the good life. I want to end this section by proposing $a$ Walzerian approach to digital media and the good life, which is built on Walzer's notion of connected criticism and his interpretative moral philosophy. Alternatively, it can be viewed as an attempt to extend the notion of connected criticism to normative analysis of digital media and the good life. Here, I shall introduce the approach by listing the five major features of the Walzerian approach.

1. Hermeneutical. To reiterate, connected criticism and Walzer's interpretative moral philosophy are about (re)interpretation of existing morality. It is, therefore, essentially hermeneutical. It starts with the assumption that the existing views of the good life have some validity; from this standpoint, the approach attempts to interrogate how the existing views of the good life make sense to us.

2. A Form of Immanent Critique. A Walzerian approach begins from within not from without, that is-it argues from the existing views of the good life, but not from some universal or objective theories of the good life. Hence, its critical force does not come externally. It criticises individual behaviours and social practices by going back to the views of the good life that are held by that person and that community. This approach, therefore, is also a form of immanent critique.

3. Participatory. A Walzerian approach is connected in that its practitioners always (seek to) engage with their people. It requires participation in current debates by paying serious attention to them and also by promptly responding to them. It is, as Walzer succinctly states, "less the practical offspring of scientific knowledge than the educated cousin of common complaint" (Walzer 1985, p. 56).

4. Empirical. A Walzerian approach is built on existing views of the good life. Hence, in an important sense, it is inherently empirical. It does not revolve around abstract theorising, but rather a study of actual behaviours, 
practices and discourses. In other words, the Walzerian approach is always based on empirical data.

5. Pluralistic. A Walzerian approach recognises the fact that cultures and societies can be different. It is this recognition that drives the approach to begin from actual behaviours, practices and discourses in order to avoid a false sense of universality or objectivity. Hence, it starts with the assumption of a plurality of views on the good life. Yet, although it does not preclude the possibility of universality or objectivity, it does insist that universality or objectivity should not be an assumption to begin with.

\subsection{What is an Appraisal of Digital Media?}

Since my approach takes seriously a specific type of empirical data, i.e. actual appraisals of digital media, the first step in developing my approach is to explicate what 'appraisal' really stands for. For the current purpose, it is particularly important to distinguish appraisals of digital media from other forms of discourse on digital media. It is helpful to start with a definition of 'appraisal' being used in a cognate discipline. Appraisal theory ${ }^{11}$, a framework developed by linguists for analysing the language of evaluation, uses the term 'appraisal' as

"a cover-all term to encompass all evaluative uses of language, including those which speakers/writers adopt particular value positions or stances and by which they negotiate these stances with either actual or potential respondents." (White 2005; my emphasis)

According to this definition, an appraisal of digital media refers to a speaker's (or writer's) positive or negative assessment of digital media and/or digitally-mediated practices. In other words, appraisals of digital media differ from other forms of discourse on digital media in that they are essentially evaluative.

My characterisation of appraisals of digital media as it stands now includes all positive and negative assessments of digital media and digitally-mediated practices. However, not all assessments are equally useful to the present study. For instance, critics may make their assessments simply by recounting their preferences, i.e. their likes and the dislikes, without giving reasons to support their assessments. Such assessments are unhelpful for two reasons. Firstly, even though personal preference

\footnotetext{
11 For an overview of the Appraisal framework, see White (2002, 2005), Martin \& White
} (2005) and Hunston \& Thompson (2006). 
may possibly reveal the underlying values and assumptions held by the person, the absence of reasons may turn an analysis of assessments into a mere speculation of values and assumptions. Secondly, and more importantly, the reasons given by critics provide the key to examining the views of the good life shared by the people. This is so because critics need to appeal to the values and assumptions shared by most, if not all, of their respondents when persuading them. By appealing to the reasons that are, and will be, shared by their respondents, the critics simultaneously reveal the values and assumptions shared by those who agree, or will agree, with them. In this way, critics and their appraisals can serve as a proxy of the views of the good life shared by the people. ${ }^{12}$ Hence, I will restrict my use of the term 'appraisals of digital media' to positive and negative assessments of digital media and digitally-mediated practices supported by reasons.

\subsection{A Taxonomy of the Appraisals of Digital Media}

The term 'appraisals of digital media' is inclusive in my current usage, i.e. it is an umbrella term for any types of appraisals ranging from scholarly to popular writings. Different appraisals of digital media are driven by distinct concerns. However, not all appraisals of digital media are equally relevant to the discussion of the relations between digital media and the good life. So, it will be useful to further narrow down the scope and focus only on those appraisals of digital media that are (directly or indirectly) related to the good life. ${ }^{13}$

According to Philip Brey, existing normative research on digital media generally falls into one of the following categories ${ }^{14}$, namely ethical analysis, normative political analysis, aesthetic analysis, and epistemological analysis ${ }^{15}$ (Brey 2007, p. 2). Brey's categorisation is based on his observation that these analyses are

\footnotetext{
12 See, section 1.5 .

13 Although appraisals of digital media can appear in either scholarly or popular venues, the present study focuses on the appraisals in popular discourse. As the appraisals reflect the views of the good life shared by those who agree with the critics, the appraisals in popular discourse, which have a larger number of potential respondents, are also a more representative window into the existing views of the good life.

${ }_{14}$ The emphasis of Brey's discussion is on the scholarly debate. But, the categories he identifies are also applicable to popular debate on digital media.

15 Brey (2007) only mentions epistemological analysis in fn. 1, but this category has become increasingly prominent in recent research, e.g. Goldman (2008), Sunstein (2006). Also, Fallis (2009) has recently edited an issue of Episteme, which is devoted to the epistemology of mass collaboration such as Wikipedia (wikipedia.org).
} 
governed by distinct normative ideals. He points out that the normative ideals for ethical analysis, normative political analysis, aesthetic analysis and epistemological analysis are the Right, the Just, the Beautiful and the True respectively (Brey 2007, p. 3). However, as he also rightly points out, the categorisation as such does not exhaust all forms of normative analysis of digital media. Brey, in particular, identifies what he labels as "cultural critique", i.e. a type of normative analysis which "[has] as [its] object [of critique] cultural practices, symbols, meanings and configurations; that is, it critiques [digital] culture" (Brey 2007, p. 4). In other words, cultural critique examines the issues generated by the development and use of digital media. In Brey's account, however, it is not the object of critique that distinguishes it from other types of normative analysis of digital media; rather it is its distinct normative ideal that makes it different. Cultural critique is different from other types of normative analysis of digital media because it is "governed by our most general ideal, which is the Good' (Brey 2007, p. 4; my emphasis).

While Brey's notion of cultural critique provides us with a useful alternative to categorise and conceptualise a family of normative analysis of digital media which does not readily fit into the four types of normative analysis of digital media originally mentioned. However, as the normative ideal of cultural critique, i.e. the Good, is the "most general ideal", the category of cultural critique appears to be too broad and too general to capture what is unique about the appraisals of the relations between digital media and the good life. For the critics, the aim of these appraisals is to communicate to the people their recommendations on digital media and digitally-mediated practices. In other words, the critics are recommending better human-digital media relations with these appraisals. In this sense, these appraisals are clearly related to the Good. However, it is not immediately clear whether these appraisals are about the good of digital media per se. It seems that the main points in these prudential appraisals are mostly limited to the actual and possible impacts of digital media and digitally-mediated practices on individuals and society. In this regards, most of them focus on which digital media and digitally-mediated practices are good for (or bad for) us, and to what extent. Accordingly, these appraisals are about the prudential values (or prudential disvalues) of digital media. Of course, the terms 'good for' (and 'bad for') and 'prudential values' (and 'prudential disvalues') can be applied to a variety of entities, e.g. people, society, environment, etc. Following James Griffin, I shall use the term 'prudential value' only to refer to "everything that makes a life good simply for the person living it" (Griffin 1996, p. 19; my emphasis). So, what distinguishes these appraisals from other normative analysis is their emphasis on prudential (dis)values or the well-being of people. 
It should be clear by now that the normative ideal governing appraisals of digital media and the good life is Well-being. Since these appraisals are guided by a unique normative ideal, i.e. Well-being, I think they merit a separate category. As these appraisals focus on prudential values (or prudential disvalues) of digital media, I shall call them prudential appraisals of digital media (or, prudential appraisals, for short).

\subsection{On Prudential Appraisals}

I have described a type of appraisals of digital media, i.e. prudential appraisals, which has Well-being as the normative ideal. Prudential appraisals may provide valuable insight into the relations between digital media and the good life insofar as they reflect the views of the good life shared by people. In other words, they are a valuable resource for the Walzerian approach that starts with existing views of the good life. However, there are two problems for any approaches that try to incorporate prudential appraisals into normative analysis of digital media and the good life. Firstly, prudential appraisals discuss what digital media and digitallymediated practices are good for us, but the term 'good for' in prudential appraisals is ambiguous. So, the meaning of prudential appraisals can be obscure. Secondly, prudential appraisals (in popular discourse) do not always explicitly argue for their claims, and many assumptions are often left unstated. Both problems can impair our interpretation and understanding of prudential appraisals. To overcome the first problem, I shall illustrate different meanings of 'goodness-for' and explain how they figure in prudential appraisals. I then provide an analysis of the structure of prudential appraisals, which, I think, can serve as a heuristic device for our interpretation and understanding of prudential appraisals.

\subsubsection{Disambiguating 'Goodness-for'}

The link between the good life (or well-being) and the notion of goodness-for should be intuitively clear. When we talk about the good life of people, we often talk about what is 'good for' them. Despite the significance of the notion of goodness-for in philosophical research on the good life, philosophical discussion on goodness (or the Good) has been preoccupied by topics related to goodness simpliciter. Major questions include, e.g. 'what is intrinsic value?', 'what is the primary bearer of value?', 'how many intrinsic values are there? And, if there is 
more than one intrinsic value, are they incommensurable or incompatible?' ${ }^{16}$ Research on goodness-for, in comparison with research on goodness simpliciter, is notably sparse. Indeed, goodness-for often appears merely as a subsidiary notion for discerning what is (and is not) good simpliciter, and little attention has been paid to the meaning(s) expressed by the term 'good for' itself. ${ }^{17}$ Recently, Connie Rosati (2009) argues that 'good for' can be used to express different relations, and not all relations expressed are about people's well-being. In other words, everyday goodfor talks are ambiguous. ${ }^{18}$ Since prudential appraisals are characterised by an emphasis on goodness-for, Rosati's conclusion raises important questions about the status of prudential appraisals, in particular: (i) what does 'good for' stand for in prudential appraisals? And, (ii) are all prudential appraisals (in popular discourse) really about people's well-being?

Against the view that, in everyday good-for talks, only a single relation is expressed by the use of 'good for', Rosati identifies five different relations the term can express, which she summarises by the following relations:

$\left(\mathrm{GF}_{\mathrm{i}}\right)$ Instrumentally Good for: $\mathrm{X}$ is good for $\mathrm{S}$ in that $\mathrm{X}$ is effective as a means to $S$.

(GFf) Good for a Use Value: $X$ is good forf $S$ in that $X$ is something it is rational to want or secure in relation to $S$, considering just what an $S$ is for.

$\left(G F_{v}\right)$ Good for an Intrinsic Value: $X$ is good for $v$ in that $X$ helps to preserve or enhance $S$ as the valuable item that it is.

(GFl) Good for a Living Thing: $X$ is good forl $S$ in that $X$ promotes the life, growth, health, or reproductive success of $\mathrm{S}$.

16 For a brief overview of the recent research on the concept of goodness, see Schroeder (2008).

17 The notion of goodness-for, I believe, receives most attention in the dependence debate, i.e. the debate on the metaphysical priority and/or the nature of goodness simpliciter and goodness-for. For example, Regan (2004) has recently revived Moore's argument against the notion of goodness-for. On the other hand, Thomson (1997) has argued that all claims about goodness should be understood in terms of goodness-for. 18 There is a different but related question concerning the ambiguity of 'good for' in everyday good-for talks, i.e. whether the ambiguity is real or only apparent? For example, Finlay (2005) has recently attempted to offer a unified semantic account of goodness via Gricean implicature. I cannot provide any decisive arguments against Finlay's account. However, following Wedgwood (2009) and Rosati (2009), I think the linguistic diversity of the term has provided, at least, prima facie support for the term's ambiguity. 
(GFw) Good for a Welfare Subject: $X$ is good forw $S$ that $X$ intrinsically or noninstrumentally benefits S. (Rosati 2009, Pp. 220-228)

In her account, $(\mathrm{GFi})$ is intended to classify those good-for talks that refer to a means-ends relation. Both $(\mathrm{GFf})$ and $(\mathrm{GFl})$, arguably, can be seen as expressing a similar relation, differing only in the type of function specified in the corresponding relation. (GFf) is best understood via the notion of good-for-an-artefact, i.e. $\mathrm{X}$ is good for an artefact $\mathrm{O}$ if $\mathrm{X}$ preserves or promotes the designed function(s) of $\mathrm{O}$; and, similarly, $(\mathrm{GFl})$ is best formulated in terms of biological function, i.e. $\mathrm{X}$ is good for a living thing $\mathrm{L}$ if $\mathrm{X}$ preserves or promotes the biological function(s) of L. ${ }^{19}$ So far, the relations elaborated are conditional, i.e. $\mathrm{X}$ is good for $\mathrm{S}$, not because of S itself; $\mathrm{X}$ is good for $\mathrm{S}$ because of some factors external to $\mathrm{S}$.

$\left(\mathrm{GF}_{\mathrm{v}}\right)$ and $\left(\mathrm{GF}_{\mathrm{w}}\right)$ describe something different. In both cases, X's being good for $\mathrm{S}$ does not require any further conditions, i.e. $\mathrm{X}$ is good for $\mathrm{S}$ as it is. And, the difference between $\left(\mathrm{GF}_{\mathrm{v}}\right)$ and $\left(\mathrm{GF}_{\mathrm{w}}\right)$ lies in the nature of $\mathrm{S}$. In $\left(\mathrm{GF}_{\mathrm{v}}\right), \mathrm{S}$ can be anything that is considered to be valuable in itself (by, at least, some persons) in the goodness-for relation, such as God in 'worship is good for God'; and, in (GFw), only those beings which are, as Rosati quotes Darwall, "capable of having a sake" can assume the position of $\mathrm{S}$ in the goodness-for relation. It is because, as she notes, when we talk about what is good for someone "we are not concerned with how things go for them for the sake of something else" (Rosati 2009, p. 226).

Let's now return to the question what does 'good for' stand for in prudential appraisals? As I have noted, the aim of prudential appraisals is to offer practical recommendations to connect oneself to (or disconnect oneself from) digital media. In putting forward a prudential appraisal, critics of digital media may use 'good for' to express $\left(\mathrm{GF}_{\mathrm{w}}\right),(\mathrm{GFf}),\left(\mathrm{GF}_{\mathrm{v}}\right)$ and $(\mathrm{GFi})$. For instance, they may comment that digital media have immediate or direct impacts on people's well-being. Hence, digital media is said to be good for (or bad for) people's well-being directly (i.e. $\left(\mathrm{GF}_{\mathrm{w}}\right)$ ). Or, they may point out that digital media will affect, or has already affected, things or practices that are deemed practically valuable (i.e. (GFf)) or intrinsically valuable (i.e. $\left(\mathrm{GFv}_{\mathrm{v}}\right)$ ). In those cases, the 'good for' is being used to refer to the

\footnotetext{
19 One can argue that both $\left(\mathrm{GF}_{\mathrm{f}}\right)$ and $\left(\mathrm{GF}_{\mathrm{l}}\right)$ denote the same relation if one holds a teleological theory of living thing (which, in turn, may also be extended to include human being; and, thereby including ( $\left(\mathrm{F}_{\mathrm{W}}\right)$, too). In my discussion, I shall ignore the complications that arise from the metaphysical statuses of artefacts, living things and human beings, as they are not directly relevant here.
} 
goodness-for the valuable things or practices at stake. Finally, for some, digital media may simply be taken as tools for specific ends. Digital media, therefore, is only instrumentally good for (or bad for) accomplishing certain ends, i.e. (GFi).

Yet, are all prudential appraisals (in popular discourse) really about people's well-being? Rosati's answer appears to be 'no'. She seems to suggest a good-for talk should be treated as a well-being talk only when 'good for' is being used to express the relation summarised in (GFw) (Rosati 2009, p. 226). However, the scope of wellbeing talks, I think, will be unnecessarily limited if we confine well-being talks only to those talks that are formulated in terms of $\left(\mathrm{GF}_{\mathrm{w}}\right)$. I think Rosati is right in paying special attention to $\left(\mathrm{GF}_{\mathrm{w}}\right)$ as the relation of well-being talks, which concern directly the subject of well-being, i.e. person; but, if we accept what is good for us is, minimally, anything that benefits us, then what we should do is to distinguish two forms of well-being talks. The first form of well-being talks is direct well-being talks, which is characterised by the relation expressed in $\left(\mathrm{GF}_{\mathrm{w}}\right)$. Then, there are indirect (or derivative) well-being talks. There are cases in which some ends, things or practices are good for the subject of well-being. If $\mathrm{X}$ preserves or promotes these ends, things or practices, our talks about $\mathrm{X}$ being good for us should be recognised as a proper form of well-being talks as well. For example, critics may claim that digital media is good for transmission of information, which, in turn, is good for knowledge acquisition. Here, if we consider knowledge to be something beneficial to people or being something that is valuable in itself, then the claim clearly belongs to well-being talks. ${ }^{20}$ If there is a clear path of reconstruction from the good-for talks in terms of $(\mathrm{GFi}),(\mathrm{GFf})$ or $\left(\mathrm{GF}_{\mathrm{v}}\right)$ to people's well-being, then, pace Rosati, the good-for talks expressing the relations in (GFi), (GFf) and (GFv) should also be included in well-being talks. ${ }^{21}$ Hence, prudential appraisals, as I have characterised so far, are indeed really about people's well-being. ${ }^{22}$

20 Some $\mathrm{S}$ being intrinsically or non-instrumentally valuable, I believe, implies that the lack of (or, a decrease in) S makes any person's well-being worse off. Hence, it is directly related to a person's well-being; but, in accordance to Rosati's analysis, I leave it in the form of indirect well-being talks. I think the reason why Rosati has separated $\left(G_{\mathrm{V}}\right)$ from $\left(G F_{W}\right)$ is because of her focus on personal good. See, Rosati (2006).

${ }^{21}$ I shall leave open the status of $\left(G_{f}\right)$ in my discussion of indirect well-being talks. There are good-for talks which are, prima facie, neither about means-ends relations nor intrinsic values, but are still relevant to people's well-being. For example,

- Digital media is good for society.

- Digital media is good for the economy. 


\subsubsection{Structure of Prudential Appraisals}

The second problem for integrating prudential appraisals (in popular discourse) arises from the implicitness of ordinary discourse. In everyday communication, it will be inefficient, if not impossible, to include all details in one's speech or writing. Some background assumptions have to be treated as the default in a communicative context. ${ }^{23}$ This mode of communication works well in everyday context. However, if we want to gain insight from prudential appraisals, we need to examine them critically. In other words, we need to explicate those values and assumptions that are treated as the default by both the critics and their respondents. However, to

These examples show that we need $\left(G_{f}\right)$ to conceptualise those good-for talks that cannot be fully captured by $\left(\mathrm{GF}_{\mathrm{i}}\right)$ and $\left(\mathrm{GF}_{\mathrm{v}}\right)$. Here, I hold that, for any good-for talks in the form of ' $S$ is good for $X$ ', they are considered as proper well-being talks if $X$ 's being better is beneficial to people.

22 There is a more fundamental question concerning the meaning of 'good for a person' which I cannot answer in full here. In his analysis, Zimmerman has examined various conceptual accounts of good-for-a-person, and he has summarised six types of conceptual accounts of good-for-a-person:

(1) Belief account of goodness-for: $\mathrm{X}$ is good for $=\mathrm{df}$. $\mathrm{P}$ believes that $\mathrm{X}$ is good.

(2) Pro-attitude account of goodness-for: $X$ is good for $P=d f$. $P$ favors $X$.

(3) Ownership account of goodness-for: $X$ is good for $P=d f$. $X$ is good and belongs to $P$.

(4) Benefit account of goodness-for: $X$ is good for $P=d f$. $X$ benefits $P$.

(5) Constrained pro-attitude account of goodness-for: $X$ is good for $P=d f$. $P$ would favour $X$, if $P$ were ideally situated in terms of both information and rationality.

(6) Deontic account of goodness-for: $\mathrm{X}$ is good for $\mathrm{P}=\mathrm{df}$. $\mathrm{P}$ has a reason to favour $\mathrm{X}$.

(Zimmerman 2009, pp. 429-430)

Following Zimmerman, I assume the correct conceptual account of what is good-for-aperson is the benefit account. As an account of good-for-a-person, both (1) and (2) suffer from the possibility of P's being ignorant about the harms of X. (3), as Zimmerman notes, results in linguistic absurdity when applied to talks about the good life, i.e. a good life is good for $P=$ df. a good life is good and it belongs to $P$ (Zimmerman 2009, p. 431; my emphasis). Finally, both (5) and (6) appear to presuppose $X$ is already in some ways beneficial to $P$, if $X$ is to be viewed as good for $P$. Hence, (4) appears to be the least controversial account amongst (1)-(6).

It should also be noted that Rosati's (and Zimmerman's) account of goodness-for is entirely conceptual. Her analysis only aims to elaborate the meaning of 'good for', but not to assert what can, or should, be identified as goodness-for. In other words, she leaves open the question: what is substantively good for a person?

23 This description of everyday communication is uncontroversial. What is controversial in philosophical, linguistic and psychological research is the nature of the default, e.g. how participants in a conversation succeed in communication without making explicit the default, etc. This is an interesting issue, but it is beyond the scope of the present study. 
critically examine prudential appraisals and the embedded values and assumptions, we need a better account of the structure of prudential appraisals. An analysis of the structure of prudential appraisals can inform us what to look at and where to look for them.

Based on the types of claims which can be found in prudential appraisals, I shall identify three dimensions of prudential appraisals. It should be noted, however, that these dimensions are neither necessary nor sufficient conditions for assessments of digital media to be qualified as prudential appraisals. It is, indeed, too demanding for critics of digital media to have all presupposed values and assumptions explicitly stated. Our interpretation and understanding of any pieces of writings or speeches depend crucially on contextual and intertextual reference. So, whether an assessment of digital media is a prudential appraisal should not be determined entirely by the explicitness or availability of those dimensions. The dimensions I outline, in other words, should be seen as a heuristic device for reminding ourselves that there are various dimensions that may, or may not, be stated explicitly in prudential appraisals.

In an evaluation of the perceived benefits and harms of the Internet, Brey (2006) has demonstrated how we should interpret and understand the claims made by critics of digital media. There are distinct dimensions in people's assessments of the Internet, as Brey summarises, “it is [people's] values systems that determine one's appraisal of technology like the Internet. [It] is also determined by one's empirical understanding of how the technology works and what its consequences or implications are" (Brey 2006, pp. 41-42). Although Brey's evaluation aims specifically at the perceived benefits and harms of the Internet, his model can be readily extended to cover prudential appraisals too. ${ }^{24}$ In other words, people's assessments of digital media, e.g. prudential appraisals, are informed by (i) their value systems, (ii) their concepts of digital media and (iii) their beliefs about the consequences or implications of digital media. Accordingly, we can isolate three

\footnotetext{
24 Brey has also distinguished two levels of analysis, i.e. descriptive analysis and critical analysis. The tasks of descriptive analysis are "(1) clarification of the meanings of concepts in the statement; (2) identification of presupposed values; (3) identification of implied empirical claims; (4) examination of the evidence for implied empirical claims". And, critical analysis goes beyond these tasks and involves a "critical assessment of values and concepts" (Brey 2006, p. 45). Accordingly, interpretation and understanding of prudential appraisals belong to the tasks of descriptive analysis, and only a normative analysis of the value dimension, the conceptual dimension and the empirical dimension will be counted as critical analysis.
} 
dimensions in prudential appraisals, and they are the value dimension, the conceptual dimension and the empirical dimension.

\subsubsection{The Empirical Dimension}

Prudential appraisals are positive and negative assessments of digital media supported by reasons, and one type of reasons is the consequences or implications of digital media. Critics discuss digital media and digitally-mediated practices because they bring, or may bring, changes to people's lives. There is no point to talk about what digital media is good for (or bad for) us, if they do not, or will not, influence people in any significant ways. Claims about the consequences or implications of digital media, therefore, form an important class of claims supporting the conclusion of prudential appraisals. I shall call these claims empirical claims.

Empirical claims can be either causal claims or correlation claims: causal claims state that a type of digital media $\mathrm{T}$ is causally related to some state of affairs A; and, likewise, correlation claims state that a type of digital media $\mathrm{T}$ correlates to some state of affairs A. And, these claims can be either factual (i.e. a type of digital media $\mathrm{T}$ causes or correlates to some state of affairs A) or possible (i.e. a type of digital media $\mathrm{T}$ will/may/can cause or correlate to some state of affairs A). To analyse these claims critically, empirical investigations are necessary. For factual claims, since they are about the state of affairs that is happening or has already happened, they can be verified or falsified by quantitative and/or qualitative research of the actual state of affairs; and, for possible claims, a critical analysis of these claims can only be done provisionally based on how probable those claims are, but the conclusiveness of the analysis will always rest on future empirical investigations.

\subsubsection{The Conceptual Dimension}

In prudential appraisals, critics of digital media will inevitably need to describe digital media, define relevant concepts, and explain how digital media works. These claims, which are about the critics' and our understandings of digital media, form another important class of claims in prudential appraisals. I shall call these claims conceptual claims. However, conceptual claims are not only limited to explicitly described, defined or explained concepts in prudential appraisals. Particularly, prudential appraisals are also based on a specific view of the nature of digital media. It contains implicit answers to questions such as 'are digital media (value-)neutral?', 'are they deterministic?', etc. So, it is important to identify the view of the nature of 
digital media in prudential appraisals, as our understandings and conceptualisations of digital media necessarily shape the way in which we assess them. Conclusions in prudential appraisals, therefore, are in part determined by critics' understandings and conceptualisations of digital media.

To critically examine our understandings and conceptualisations of digital media, we need to refer to the research in philosophy of technology. As Lucas Introna (2008) helpfully summarises, there are three major views on the nature of technology offered by philosophers of technology, which he labels "the impact view", "the constructivist view" and "the phenomenologist view".

The impact view takes technology simply to be an artefact or tool for a certain objective and outcome, the view's emphasis on objective and outcome most often links to the impacts of technology, as an artefact or tool, on people. As such, the view also assumes technology to be neutral. While this view does not necessarily imply a technology $\mathrm{T}$ functions uniformly across different contexts, it does imply that T's outcome is, to a certain extent, determined in a given context. Therefore, it is closely related to technological determinism, which is, roughly, the idea that technology is the cause of social changes. On the other hand, the constructive view takes technology to be an outcome of continuous negotiation between different actors/actants, including the technology itself, in a society. Therefore, in the constructivist view, technology and society co-construct each other. In the coconstruction, some uses and designs are favoured over the others. Since the favoured uses and designs represented certain preferences; in this sense, technology is not neutral, but value-laden. Finally, the phenomenologist view asserts that technology and society cannot be examined in isolation, because, as Introna put, "they are each other's ongoing condition or possibility for being what they are" (Introna 2008). In the phenomenologist view, manifestations of technology reflect specific attitudes towards the world and, at the same time, technology itself also mediates between human being and the world. Since specific attitudes (towards the world) are entrenched in technology, technology cannot be considered as valuefree too.

Since people's views of the nature of technology play an important role in shaping their assessments of digital media, an erroneous understanding or conceptualisation of digital media may affect the plausibility of prudential appraisals. A thorough critical analysis, therefore, has to pay special attention to the view of the nature of technology presupposed in prudential appraisals. 


\subsubsection{The Value Dimension}

Prudential appraisals are, in essence, normative and evaluative. The normativity of the conclusions in prudential appraisals is derived from value claims given by critics. These value claims are normative and evaluative in that they contain normative and evaluative terms such as 'good', 'valuable', 'well-being', etc. ${ }^{25}$ There are, roughly, two ways to express value claims in prudential appraisals, i.e. they can be expressed explicitly or derivatively. ${ }^{26}$ For instance, they can be formulated explicitly in the following forms:

(V1) X is good/valuable (for S)

(V2) $\mathrm{X}$ is bad (for $\mathrm{S}$ ).

Alternatively, they can also be expressed derivatively in the following forms:

(V3) (i) Y has evaluative properties E1...n, and (ii) E1...n are good/valuable (for $\mathrm{S}$ ).

(V4) (i) Y has evaluative properties E1...n, and (ii) E1...n are bad (for S).

Of course, these forms are only approximations of the value claims in prudential appraisals. In particular, (ii) in derivatively expressed value claims may be left unstated by critics. Hence, an important task for a critical analysis of value claims is to uncover the implicit part(s) of these value claims and to identify what values are in prudential appraisals. But, identifying the unstated and/or implicit values is only the first step of a critical analysis of value claims. A thorough critical analysis must also answer the questions: (i) what is the basis of the critics' values? and also, (ii) is the basis of those values valid?

Critics of digital media, in presenting their prudential appraisals, may refer to a set of values, which is held to be universal, to justify their conclusions. Alternatively, they may appeal to a particular culture, usually their own culture, to be the basis of their value claims. In other words, the basis of their value claims can either be a set of universal values or it can have its root in a particular culture. And, their validity is, ultimately, determined by a plausible account of the nature of the good life. If there is one universal value or one set of universal values, which is

\footnotetext{
25 Since prudential appraisals concern exclusively the relations between digital media and the good life, I will only mention the values and evaluative properties that are related to the good life here. But, I think the approach proposed in this chapter can be easily extended to cover other values and evaluative properties as well.

26 They correspond to the two forms of well-being talks, i.e. the direct well-being talks and the indirect (or derivative) well-being talks, I have described in section 1.4.1.
} 
constitutive of people's well-being exhaustively, then a true systematised theory of the good life will be able to tell us what those values are and why they ought to be maintained, and also to allow us to verify and falsify value claims. If, on the other hand, the views of good life differ from one culture to another, then the validity of the critics' value claims has to be checked against a particular culture. It, then, requires investigations into the sets of values shared by people in different cultures. The sets of values have gone by different names, but I shall call it practical identity. ${ }^{27}$

There are different assessments of digital media regardless of whether they are, in the end, correct or not. By looking at the value dimension of prudential appraisals, it is possible to see how different people come to assess digital media in a specific way. At the same time, it can also illustrate the relations between digital media and the good life held by the people (from different cultures and societies), which, I think, is indispensible in normative analysis of digital media and the good life. ${ }^{28}$

\subsection{Prudential Appraisals and the Good Life}

The normativity of prudential appraisals, as I have argued, is based on value claims, which, in turn, presuppose specific views of the good life. Yet, whether the views of the good life (in prudential appraisals) are universal or sociocultural-specific remains to be discussed. This is an important question because the nature of the good life in prudential appraisals has implications for selecting the appropriate approach for the present study. In this section, I discuss the nature of the good life in prudential appraisals and explain how it lends support to the Walzerian approach. But, in order to illustrate the nature of the good life in prudential appraisals, a deeper look at people's practical and prudential deliberation is first required.

27 Following Rawls, Brey (2006) calls the set of shared values, which is also called value systems or ideologies, comprehensive doctrines. He states that "[c]omprehensive doctrines are systems of value, be they religious, moral or ideological, that contain values concerning what is good and bad, and are often accompanied by norms for conduct and a system of (metaphysical) beliefs." Here, I opt for the term 'practical identity' because of the intimate connection it has to practical and prudential deliberation. I shall return to this in Section 1.5.

28 Here, the term 'value dimension' is intended to provide a broad category to capture two distinct but related forms of analysis of value, i.e. conceptual analysis and normativeevaluative analysis. My use of 'value dimension' is similar to the use of 'ethics' as an umbrella term for meta-ethics and normative ethics. 


\subsubsection{Practical Identity and the Good Life}

What digital media is good for (or bad for) us depends on who we are. After all, people have different experiences, capacities and expectations. Differences in these factors will surely affect the answer to such a question. Yet, important as these factors are, they only provide external considerations for people to determine what digital media is good for (or bad for) them. And, by themselves, these factors provide neither motivating nor normative reasons for people to adopt certain human-digital media relations. There is, however, another sense of 'us', which provides motivating and normative reasons for people to relate to digital media in specific ways. This sense of 'us' is our identity, or as David Wong characterises it, it is people's "ways of understanding and interpreting the world, their characteristic ways of behaving and relating to other people, their views as to what is most important and valuable in life, and what are in fact their most important ends and desires" (D. Wong 2006, p. 118; my emphasis). ${ }^{29}$ This sense of who we are instructs us why and how we ought to relate to digital media in such-and-such a way by measuring up our relations to digital media with "what is most important and valuable in [our] life, and what are in fact [our] most important ends and desires". Who we are, in other words, provides us a standard for our practical and prudential deliberation.

The sense of 'us' in David Wong's account, however, is too general. Particularly, the role of 'us' in practical and prudential deliberation needs to be unpacked more clearly. ${ }^{30}$ The best way to unpack this sense of who we are and its role in practical and prudential deliberation, I think, is through the notion of practical identity proposed by Christine Korsgaard (1996). Practical identity, I shall illustrate, provides the link between people and their views of the good life; and, make the views truly theirs. This link, then, provides motivating and normative

\footnotetext{
29 So construed, 'identity' and 'comprehensive doctrine' are closely related, i.e. the term 'identity' refers to a set of beliefs, norms and values that have been apprehended by people, as part of themselves, whereas the term 'comprehensive doctrine' refers to a set of beliefs, norms and values available to people to apprehend. Identity, therefore, can be viewed as a personalised manifestation of an objective or inter-subjective comprehensive doctrine.

30 David Wong's (2006) discussion of identity is far more detailed than what I have quoted. His analysis of identity is both philosophical and psychological. Without going into the details of his analysis, I think D. Wong's account is largely in agreement with Korsgaard's notion of practical identity. I cite his general description of identity here because it expresses the commonsensical meaning of 'identity' most lucidly.
} 
reasons for their practical and prudential deliberation. Furthermore, I shall argue that the notion of practical identity is necessarily sociocultural. If I am right about the sociocultural nature of practical identity, people's practical and prudential deliberation is socially and culturally bound too. It is, therefore, also relevant to which approach is more appropriate in examining the relations between digital media and the good life.

\subsubsection{Korsgaard on Practical Identity}

I shall begin by elaborating Korsgaard's notion of practical identity and explain its role in practical and prudential deliberation. I then connect the notion of practical identity to the normativity of prudential appraisals. But, a clarification has to be made first. Korsgaard's discussion of the notion of practical identity is centred on what she calls "the normative question", i.e. "[w]hy should I be moral [...] We are asking what justified the claims that morality makes on us" (Korsgaard 1996, pp. 910; my emphasis). Given her emphasis, the notion of practical identity is being used in grounding moral reasons and obligations. ${ }^{31}$ Moral reasons and obligations in Korsgaard's sense, however, are not the focus of this chapter. Recall the distinction between 'what is the just and right thing to do?' on one hand and 'what is the better thing to do?' on the other hand; only the latter is my focus here. In other words, my aim is to show how the notion of practical identity can ground (positive) recommendations related to people's well-being. ${ }^{32}$ More specifically, my aim is to illustrate the role of practical identity in prudential appraisals. To juxtapose Korsgaard's "normative question", my question, which I shall call the ameliorative question, is that: why should I live better, and what justifies the recommendations being made on us?

${ }^{11}$ More precisely, Korsgaard uses our practical identity as human beings to ground moral reasons and obligations. See, Korsgaard (1996, Chapter 3).

32 Interestingly, Korsgaard starts The Sources of Normativity noting the two sets of ethical standards, i.e. the right and the good. She states, "[morality] make claims on us: they command, oblige, recommend, or guide. Or at least, when we invoke them, we make claims on one another. When I say that an action is right I am saying that you ought to do it; when I say that something is good I am recommending it as worthy of your choice" (Korsgaard 1996, p. 8). Unfortunately, she rarely discusses the good in The Sources of Normativity. It seems that she is indifferent to terms such as 'command' and 'oblige', which usually connote with 'the just' and 'the right', and terms such as 'recommend' and 'guide', which connote more often with 'the good' (and, in particular, 'prudential good'). 
Whether or not Korsgaard's notion of practical identity has successfully answered "the normative question", I think the notion offers a plausible answer to the ameliorative question. According to Korsgaard, practical identity is

"a description under which you value yourself, a description under which you find your life to be worth living and your actions to be worth undertaking... [It $]$ is a complex matter and for the average person there will be a jumble of such conceptions. You are a human being, a woman or a man, an adherent of a certain religion, a member of an ethnic group, someone's friend, and so on. And all of these identities give rise to reasons and obligations. Your reasons express your identity, your nature; your obligations spring from what that identity forbids." (Korsgaard 1996, p. I0I; my emphasis)

Korsgaard argues that reasons and obligations arise from one's practical identity. She points out that violations of people's most important practical identity will result in a loss of integrity and self-identity that "[the people will] no longer be able to think of [themselves] under the description under which [they] value [themselves] and find [their] life to be worth living and [their] actions to be worth undertaking. It is to be all practical purposes dead or worse than dead" (Korsgaard 1996, p. 102). In other words, people's maintenance of their integrity and selfidentity requires them to follow what is prescribed by the practical identity most important to them. In this way, people's practical identity defines their obligations.

The relation between reasons and practical identity in Korsgaard's view, however, is less direct. Korsgaard argues that as reflective beings, we "need a reason. Otherwise, at least as long as [we reflect, we] cannot commit [ourselves] or go forward [...] We need reasons because our impulses must be able to withstand reflective scrutiny. We have reasons if they do" (Korsgaard 1996, p. 93). The question here, of course, is when have our impulses withstood reflective scrutiny. At this point, the notion of practical identity steps in: an impulse has withstood our reflective scrutiny when it accords to our practical identity; it is, then, also a reason for us to deliberate or to act. Reflective scrutiny via practical identity, in Korsgaard's view, gives us reasons and, thereby, tells us what we are allowed to do.

So far so good, but I think Korsgaard has too closely tied reasons, either for deliberation or for action, with obligations. For instance, she states that "when we do reflect we cannot but think that we ought to do what on reflection we conclude we have reason to do. And when we don't do that we punish ourselves, by guilt and regret and repentance and remorse" (Korsgaard 1996, p. 104). So construed, like 
obligations, which specify what is prohibited, reasons merely express what people are obliged, that is-what they are prohibited not to do.

Korsgaard's notion of practical identity has left out something important, or so I argue. What is missing in Korsgaard's notion of practical identity is the relation between recommendations and practical identity. The omission is significant because, like reasons and obligations, recommendations are normative, i.e. they demand people to choose or to do what is being recommended and refrain from what is being recommended not to do. But, unlike reasons and obligations, they cannot be conceptualised exhaustively in terms of what is allowed or prohibited by people's practical identity. Of course, there are negative recommendations, i.e. those recommendations which recommend people not to choose or to do something, which may be grounded in what people's practical identity forbids. ${ }^{33}$ But, there are also positive recommendations, i.e. those recommendations which recommend people to choose or to do something. It is these types of recommendations, I think, cannot be grounded in what is allowed or prohibited by people's practical identity.

Recommendations, especially positive recommendations, have to do with choices or actions that are optional in the sense that they are neither impermissible nor required; but, loosely defined, any trivial choices people have made, or any mundane actions people have performed, can be viewed as optional. Hence, I want to use the terms 'optional choice(s)' and 'optional action(s)' technically to refer to the types of choices and actions that are neither impermissible nor required but nonetheless are significant to people's well-being. So construed, there are two types of optional choices and actions. Firstly, there are optional choices and actions that are good for people's well-being but are not required. Likewise, there are optional choices and actions that are bad for people's well-being but are not impermissible. Against this setting, then, recommendations can be viewed as normative claims concerning optional choices and actions. Positive recommendations are about optional choices and actions that are good for a person but are not required. They assert that people's well-being will be better off following what is being recommended; and, negative recommendations are about optional choices and actions that are bad for a person but are not impermissible. They assert that people's

33 Here, I only mean that some but not all negative recommendations can be grounded in what people's practical identity forbids. Since there are negative recommendations on choices and actions that are neither impermissible nor required; and, similar to positive recommendations, these negative recommendations cannot be conceptualised in terms of what people's practical identity forbids, too. 
well-being will be worse off if they do not follow them. ${ }^{34}$ Still, recommendations are normative, as I have pointed out, in the sense that they demand certain choices or actions.

Intuitively, positive recommendations go beyond what people are allowed to do in that they encourage them to do what is being recommended. In other words, they are stronger than Korsgaard's reasons as what-is-allowed in that they demand people to choose or to do what is being positively recommended. Hence, they should not be taken to be the same as reasons, as they are characterised by Korsgaard. Yet, I think, neither should we take them simply as obligations. Obligations, as Korsgaard states, are grounded in what people's practical identity forbids. In order to ground positive recommendations on what their practical identity forbids, we have to (re)phrase positive recommendations in a double negative, that is-it is forbidden to not meet the positive recommendations.

Such a formulation, however, is unappealing. Firstly, consider a possible scenario of a person having more than one reasonable, but conflicting positive recommendations. ${ }^{35}$ In this scenario, even if this person follows one of the positive recommendations, the formulation still forces us to conclude that this person has given up his integrity and self-identity because he has failed to follow at least one of the positive recommendations. Secondly, the formulation also requires us to conclude that a failure to meet what is positively recommended to be devastative to his integrity and self-identity. Yet, not only does this conclusion appear too strong, it also seems to get the point of positive recommendations wrong. The point of positive recommendations is to provide people a sense of direction, i.e. a sense of what they should care about, failures to meet positive recommendations may indeed incur some senses of loss and uneasiness, but they should not lead to selfdisintegration and self-rejection because of the directionality provided by positive recommendations.

\footnotetext{
34 As I have noted, negative recommendations may not be exhaustively conceptualised in terms of what people's practical identity forbids. But, the possibility of negative recommendations being conceptualised in terms of what people's practical identity forbids suggests an asymmetry between negative recommendations and positive recommendations. To avoid further complications, I shall restrict my discussion only to positive recommendations.

35 Here, my argument does not depend on a specific type of conflict; it goes so long as the conflict prevents the person from following one of the positive recommendations. In other words, the conflict can be logical, practical or even temporal.
} 
Positive recommendations, therefore, should also be distinguished from obligations. Although positive recommendations cannot be grounded in what is allowed or prohibited by a person's practical identity; I think, a more positive reading of the notion of practical identity can still help us to conceptualise them. I propose to supplement Korsgaard's doublet of reasons and obligations with another member, i.e. (positive) recommendations, which flow from what people's practical identity strives for. Practical identity, in short, specifies what people are allowed, prohibited, obliged and encouraged (and, for negative recommendations, discouraged) to choose and to do. ${ }^{36}$

Practical identity can undertake this role because it has embodied a set of restrictions - moral or non-moral—for and prior to people's deliberation and action. Harry Frankfurt (1999b) has convincingly argued that this prior set of restrictions is conceptually necessary for deliberation and action. He argues that without a prior set of moral or non-moral restrictions, people's decisions and actions cannot truly be regarded as their own. He invites us to imagine a person, who is free of any restrictions on choices and actions, and who can even "redesign his own will" for any choices and actions. Frankfurt points out, in this scenario, since that person can alter his will to fit any choices and actions, his decisions and actions are guided by no "definitive set of goals, preferences or other principles of choice." But, as Frankfurt also notes, in this scenario, any of his decisions and actions cannot fully or wholeheartedly be regarded as his own because those choices he made or those actions he performed are "merely adventitious and provisional, for he has not committed himself to them and can alter them as he wants." In other words, for a choice or an action to be seen as truly or wholeheartedly his, it must be rooted in some restrictions of choices and actions that the person has accepted antecedently. Restrictions are necessary, as Frankfurt summarises, because "someone free of all restrictions is so vacant of identifiable and stable volitional tendencies and constraints that he cannot deliberate or make decisions in any conscientious way" (Frankfurt 1999b, pp. 109-110).

${ }^{36}$ In a similar vein, Bransen (2000) argues that there is a set of practical problems that cannot be sufficiently answered by morality (i.e. what is allowed, prohibited and obliged) and rational choice theory (i.e. utility, consequences, etc.) but by the notion of alternatives of oneself (i.e. who should I be?). Here, I think my emphasis on (positive and negative) recommendations is largely in agreement with Bransen's view. One way to reformulate the current discussion in Bransen's terms, therefore, is to view the purpose of (positive or negative) recommendations is to offer individuals alternatives of oneself. Also, see Bransen (2002, 2008). 
The lesson from Frankfurt's story is that practical identity, in grounding reasons, obligations and recommendations, must embody a set of restrictions. The question, therefore, is what those restrictions are. Frankfurt's answer is that the restrictions are things that we care about and things that we regard as important to us (Frankfurt 1988). ${ }^{37}$ Frankfurt's notions of what we care about and what is important to us has generated numerous complex and intriguing arguments, which I will not repeat here. Leaving aside the details of Frankfurt's account, it is relatively clear that Frankfurt's notions of what we care about and what is important to us are merely structural, i.e. they only describe a (formal) relation between people and things. ${ }^{38}$ In other words, they have no answer to the question concerning the restrictions substantively; or, in Frankfurt's own terms, they only affirm that human beings must care about something and that they must regard something as important to them. But, the notions do not tell us what there is that we care about or what there is that is important to us.

What we do care about and what we do regard as important to us, of course, are empirical questions. And, it is true that there is a diverse range of matters that we $d o$ care about and that we do regard as important to us. Hence, without limiting the scope of inquiry, the substantive questions concerning the restrictions are not manageable. Here, I will try to answer the substantive questions by means of reverse reasoning, that is- I begin from the conclusion that our practical and prudential deliberation pertaining to major issues relate to the good life is, in fact, based on what we care about and what we regard as important to us, and then I try to offer the best candidate, in my mind, that provides the restrictions for and prior to people's practical and prudential deliberation.

37 In his more recent writings, Frankfurt has replaced what we care about and what we regard as important to us with the notion of love. See, Frankfurt (1999a, 2004, 2006).

$3^{8}$ For example, Frankfurt writes,

"When a person cares about something, [...] he is willingly committed to his desire.

The desire does not move him either against his will or without his endorsement [...]

On the contrary, he himself desires that it move him. He is therefore prepared to intervene, should that be necessary, in order to ensure that it continues.

Besides wanting to fulfil his desire, then, the person who cares about what he desires wants something else as well: he wants the desire to be sustained. Moreover, this desire for his desire to be sustained is not a merely ephemeral inclination. It is not transient or adventitious. It is a desire with which the person identifies himself, and which he accepts as expressing what he really wants." (Frankfurt 2004, pp. 15-16) 
Already hinted at in my discussion of the relation between recommendations and practical identity, i.e. recommendations are what people's practical identity strives for, and, also to be found in Frankfurt's speculative answer to the substantive aspects of what we should care about and what should be taken to be important to us (Frankfurt 1999b, pp. 114-115), I suggest it is the ideals embodied in people's practical identity that provide the required sets of restrictions.

To illustrate how the ideals embodied in practical identity provide a set of restrictions which underlie people's practical and prudential deliberation and ground (positive) recommendations, I shall now turn to David Wong's account of identity (D. Wong 2006, Chapter 4). Based on extensive evidence from psychological and anthropological research, David Wong conceptualises identity in terms of traits. He identifies four categories of traits which, together, constitute people's identity. The four categories of traits include (i) somatic dispositions, (ii) temperamental or psychological traits, (iii) social role traits and socially defined group traits and (iv) ideal traits. ${ }^{39}$ And, what is relevant for the current purpose is his discussion of the role of ideal traits. He writes,

"Ideal traits set directions for the development of actual traits [...] people's conceptions of ideal selves can affect both the types of actions they perform and their manner of performing them. Because the ideal self defines a set of general ends and values, it affects the details of practical deliberation and directions of choice, often by determining what is salient." (D. Wong 2006, p. 123)

Since an ideal self has defined "a set of general ends and values", through this set of ends and values, recommendations become sensible to the people who take up this ideal identity; or, again, in Frankfurt's terms, recommendations become binding to people because it is what the people cares about. As David Wong has also pointed out, "[i]deals are often the best explanation of a person's having acquired or

39 David Wong (2006) separates quite sharply between socially defined role and group traits (or role and/or group identity) and ideal traits (or ideal identity). The sharp separation, I think, is unnecessary for the current discussion. One way to put it is that: ideal identity is essentially social, and social identity essentially involves some ideals. Firstly, as he notes, people's ideal identity arises from the community where they belong. So construed, identifying oneself with an ideal identity will always be a social process. Secondly, if a social identity can inform people's choices and actions, as David Wong appears to suggest, then it must include some standards for being seen as in that role or group; and, a social identity, in specifying what it is for people to be in a role or a group, will always embody some ideals. Also, see Appiah (2005, pp. 21-23, 65-71). 
developed a pattern of desires. And, similarly, group or role identity can often provide the best explanation of a person's holding certain beliefs" (D. Wong 2006, p. 124). If his account of the role of ideal traits (or, as I prefer, ideal self) is correct, then ideals appear to be the best candidate that provides the set of restrictions for and prior to people's practical and prudential deliberation.

To conclude this section, two further remarks concerning ideals have to be made. Firstly, I have remained silent on the metaphysical status of ideals, especially whether ideals are mind-dependent or mind-independent, because, I think, such a question is irrelevant to the present study. Still, it is important to note that an ideal cannot have any imports if people do not identify it to be their own. Hence, ideals are always personal in the sense that they are, and have to be, embodied in people's practical identity. But, the personal aspect of ideals is only part of the story, people do not construct their practical identity ex nihilo; they acquire their ideals from and in a given sociocultural context during their identity formation. Hence, my second remark: people's practical identity and the ideals it embodies are essentially sociocultural; and, I shall now turn to illustrate in more detail the sociocultural nature of practical identity.

\subsubsection{Practical Identity and Its Sociocultural Nature}

I have remarked that practical identity and the embodied ideals are both personal and sociocultural. ${ }^{40}$ In this section, I discuss in detail what it means to say that people's practical identity and the embodied ideals are sociocultural. Since my discussion draws heavily from the communitarian idea of the self, I first explain the main tenet of the communitarian self, and then I illustrate how the communitarian self provides insight into the sociocultural nature of practical identity and the embodied ideals.

As Daniel A. Bell (2010) notes, communitarianism can be seen as a response to liberal political theory as exemplified by John Rawls' A Theory of Justices (1972). Bell has helpfully documented various topics of dispute between the two sides, but I shall only concentrate on the communitarian idea of the self. The communitarian

\footnotetext{
40 Recently, the temporal and/or historical nature of practical identity has received much attention in the discussion of autonomy and other related issues; but, to avoid further complications, I shall leave aside the temporal and historical nature of practical identity in my discussion. It is enough to note that what I shall argue is compatible with the claim that practical identity is necessarily temporal and/or historical. See, e.g. Christman (2009).
} 
idea of the self arises from the critique of the liberal self, which is labelled by the critics as "the atomistic self". The crux of the liberal self, according to communitarians, is that the liberal self is fundamentally self-determining and that it is prior to and detachable from any social and cultural commitments. In response to this idea of the self, communitarians argue that the social and cultural commitments do not come after the self. Instead, they argue that the self is structured by people's social and cultural commitments; and, therefore, those commitments are constitutive of our self-formation and self-understanding.

However, the debate on the nature of the self between the two sides is well worn; it is not too controversial to assert that, emerging from the debate, there is a general consensus on the truth of the social and cultural nature of the self. The disagreement between the two sides, as it turns out, does not rest on their different concepts of the self. Instead, the disagreement turns to the degree over which the social and cultural commitments enter into people's self-formation and selfunderstanding. ${ }^{41}$

The communitarian critique of the liberal self is certainly a timely reminder of the fact that human beings are sociocultural beings. But, beyond this general assertion about human nature, what else can we learn from this critique? I think the communitarian critique has also offered us insight into the nature of ideals, i.e. the normative standards for people's practical and prudential deliberation. In arguing human beings are essentially structured by their social and cultural commitments, communitarians insist our moral and non-moral judgements are also confined by our culture and society, because the standards of our moral and non-moral judgements, i.e. the ideals, are too entrenched in a given social and cultural context.

What we can learn from the communitarian critique, therefore, is that we can conclude from the sociocultural embeddedness of human beings the sociocultural nature of practical identity and the embodied ideals. Various arguments have been put forward in favour of the communitarian self, I shall restrict my discussion to the argument concerning the precondition of self-formation and self-understanding powerfully formulated by Charles Taylor. ${ }^{42}$ Taylor has argued for the sociocultural nature of the self through an analysis of the use of language, to quote Taylor rather extensively, he writes,

\footnotetext{
41 Cf. Christman (2009, Chapter 2).

$4^{2}$ Cf. Walzer (1993).
} 
"There is no way we could be inducted into personhood except by being initiated into a language. We first learn our languages of moral and spiritual discernment by being brought into an ongoing conversation by those who bring us up. The meanings that the key words first had for me are the meanings they have for us, that is, for me and my conversation partners together. Here a crucial feature of conversation is relevant, that in talking about something you and I make it an object for us together, that is, not just an object for me which happens also to be one for you ,even if we add that I know that it's an object for you, and you know, etc. The object is for us in a strong sense, which I [describe] with the notion of 'public' or 'common space'. The various uses of language set up, institute, focus, or activate such common spaces, just as it would appear the very first acquisition of language depends on a proto-variant of it, as seems indicated in the pioneering work of Jerome Bruner.

So I can only learn what anger, love, anxiety, the aspiration to wholeness, etc., are through my and others' experience of these being objects for us, in some common space... I cannot clarify what I feel until I talk about it with certain special partner(s), who know me, or have wisdom, or with whom I have an affinity...

This is the sense in which one cannot be a self on one's own. I am a self only in relation to certain interlocutors: in one way in relation to those conversation partners who were essential to my achieving selfdefinition; in another in relation to those who are now crucial to my continuing grasp of languages of self-understanding-and, of course, these classes may overlap. A self exists only within what I call 'webs of interlocution'." (Taylor 1989, pp. 35-36)

Taylor's argument is rested on the claim that (i) languages are socially and culturally specific (and socially and culturally constructed). And, (ii) values and commitments are framed by our linguistic repertoire. So, (iii) our values and commitments are necessarily social and cultural. Finally, (iv) since people only acquire values and commitments through languages and their interactions and/or communications with others, what they can acquire must too be socially and culturally specific (and socially and culturally constructed). Although Taylor does affirm the claim that the self is exhaustively determined by its social and cultural background, I think, the determination can be a weak one, i.e. the self is determined by its social and cultural background in the sense that it must be defined and maintained in light of 
the values and commitments available within a given social and cultural background; it does not, however, specify which background or, for that matter, how many backgrounds, a self must be situated. In other words, it does not preclude the possibility of self-transformation, i.e. to give up those ideals a person currently holds and to pick up new and unfamiliar ideals; what it precludes is the thought that self-transformation can take place in a vacuum. Accordingly, selftransformation must take place in connection to other social and cultural backgrounds.

Now, it should not be difficult to see how ideals are socially and culturally specific (and socially and culturally constructed) in Taylor's sense. Here, if we see ideals as (quasi-)coherent sets of basic values and commitments which specify what a person should strive for, then ideals can only enter into a person's identity through interaction and communication with languages. Hence, like any values and commitments, people's ideals are fundamentally social and cultural, too.

\subsubsection{From Personal Judgements to Prudential Appraisals}

Practical identity, with the ideals it embodies, informs people what is recommended (and what is not recommended); or, alternatively, it specifies what a person should and should not care about. In other words, it is the normative basis of practical and prudential deliberation. People's judgements of the impacts of digital media and digitally-mediated practices on their well-being, therefore, may also be understood in terms of what is recommended by their practical identity. If a particular type of digital media (or a specific human-digital media relation) is commendable from the standpoint of what they care about, then it will be judged favourably and positively; and, it will be judged otherwise if it is not. In this respect, prudential appraisals are not too different from personal judgements, i.e. prudential appraisals are also grounded in practical identity and the embodied ideals. But, there is one crucial difference between prudential appraisals and personal judgements of digital media and digitally-mediated practices, that is-practical identity and the embodied ideals in prudential appraisals must be those that are, or can be, shared by people.

In presenting prudential appraisals, critics aim to demonstrate what digital media is good for (or bad for) their respondents. Hence, like any person, critics need a normative standard for their judgements, i.e. the prudential appraisals. The normative standard can be found in any of the practical identities endorsed by the critics. However, not every practical identity the critics endorse can be the normative basis of prudential appraisals because prudential appraisals are not 
merely personal judgements, they are essentially public. As I have pointed out, the aim of critics is to persuade their respondents that their judgements and recommendations in their prudential appraisals provide better ways, if not the best ways, to relate to digital media. Here, those judgements and recommendations are intended to be motivating and normative.

It is only when the critics' respondents take the practical identity which the critics endorse in their prudential appraisals to be their own, recommendations can attain this motivating and normative status. For it is only then that practical identity does matter to the critics' respondents. So construed, the practical identity in prudential appraisal has to be shared by the people. In other words, critics of digital media have to align themselves with their respondents: they do not only speak to their respondents, they also speak as their respondents. When critics of digital media speak as the people, prudential appraisals can be seen as a form of collective (personal) judgements on the impacts of digital media and digitallymediated practices on their well-being.

\subsubsection{The Walzerian Approach Revisited}

I have argued that people's judgements on the impacts of digital media and digitally-mediated practices depend crucially on who the person is because there are always some ideals embodied in a person's (practical) identity. And, I have also argued that recommendations derived from people's practical identity are motivating and normative because people have already identified with the ideals embodied in that practical identity which grounds those recommendations. Two lessons can be drawn from this discussion. The first lesson is more controversial, and it is about the nature of our judgements pertaining to the relations between digital media and the good life. The second lesson is less controversial, and it is about a methodological consideration for the present study. Both lessons, I think, lend support to the Walzerian approach I have outlined. Now, I shall discuss the two lessons in turns.

If the preceding discussion concerning the sociocultural nature of ideals is correct, it entails that our judgements pertaining to the good life will too be essentially socially and culturally specific. Given that normative analysis of the relations between digital media and the good life is a species of judgements and recommendations, they too must be socially and culturally specific. Furthermore, if normative analysis of digital media and the good life aims to persuade people to relate to digital media in specific ways, then they too must be based on practical identity and ideals that are shared, or can be shared, by the people. As such, 
normative analysis of digital media and the good life does not differ from prudential appraisals. In short, the more controversial lesson from the previous discussion is that: normative analysis of digital media and the good life has to be socially and culturally specific, that is-it must deploy those ideals available within a specific culture and society. As such, normative analysis of digital media and the good life has to be interpretative.

Since ideals are socially and culturally specific and prudential appraisals are based on these socially and culturally specific ideals, in order to interpret and understand prudential appraisals properly, we should begin by looking at the ideals embedded in practical identity within various cultures and societies. So, the less controversial lesson from the previous discussion is that: for a proper understanding and interpretation of prudential appraisals, we should start by studying the existing views of the good life instead of starting from a universal or objective theory of the good life. So, the best way to proceed, as I have attempted to show, is through an interpretative approach such as the Walzerian approach.

\subsection{Why Do We Need Prudential Appraisals of Digital media?}

In previous section, I have argued that the Walzerian approach to digital media and the good life is a viable approach for the present study. Before ending this chapter, I want to offer an additional argument for the indispensability of a major component in this approach, i.e. prudential appraisals. I shall show that abstract theories alone, e.g. philosophical theories of the good life, are by themselves insufficient for normative analysis of the good life and, thus, fail to shed light on the relations between digital media and the good life. I argue that an adequate normative analysis has to acknowledge the particularity of the good life. In short, abstract theories are only useful when they are supplemented by prudential appraisals. Presenting this additional argument will, I hope, reinforce the importance of prudential appraisals and the need for an interpretative approach similar to the Walzerian approach.

My strategy for the additional argument will be as follows: I shall restrict the scope of my argument to philosophical theories of the good life, and I assume their importance in normative analysis of the relations between digital media and the good life. I then show that any normative analysis that is primarily based on philosophical theories of the good life is incomplete without also taking into account the actual discourse seriously, i.e. prudential appraisals.

Abstract theorising, of course, is not only practised in philosophy. For instance, psychological research on the good life (and well-being) also involves abstract 
theories (see, e.g. Tiberius 2006). However, abstract theorising is prototypical to philosophical discussion on the good life. Hence, philosophical theories of the good life are good candidates to illustrate the inadequacy of abstract theorising. Although I restrict my discussion to philosophical theories of the good life here, I think it can readily be generalised. ${ }^{43}$ Moreover, it should be noted that I intend my argument to be against any normative analysis which prioritises theory over empirical data. In this sense, my argument is also targeted at some of the approaches to normative analysis that take into account empirical data, but insufficiently so, e.g. the method of reflective equilibrium. ${ }^{44}$ Before proceeding to my argument, however, it is helpful to first revisit the major philosophical theories of the good life.

Philosophical theories of the good life generally fall into one of the following types: hedonism, desire theories and objective list theories. ${ }^{45}$ Hedonism, in its simplest formulation, maintains that the more pleasure and the less pain a person has, the better will be the person's life. According to hedonism, a good life is one in which a person maximises pleasure and minimises pain. A naïve desire theory, on the other hand, holds that a person's good life consists of satisfaction of his desires. In other words, a person's life is at its best if he can satisfy all of his desires. Finally, an objective list theory takes the good life to be determined by a list of goods that is independent of a person's acquisition of pleasure or satisfaction of desires. An objective list theory is so called because the list of goods specified in the theory is required by any person's good life, regardless of who the person is. Typically, knowledge, friendship and other virtuous characters are on the list. Accordingly, people have a good life when they have most (or all) of the goods on the list.

My aim in describing the philosophical theories of the good life is to illustrate their expected role in normative analysis of the relations between digital media and the good life. If philosophical theories are by themselves sufficient for the analysis, then assessments of digital media and digitally-mediated practices powered by a particular philosophical theory of the good life can already deliver normative

\footnotetext{
43 Tiberius's (2006) discussion is particularly helpful here, because she has mapped out the similarities and differences between philosophical and psychological research in the good life (and well-being). Also, see Gasper (2006), for an overview of various conceptualisations of well-being and different approaches to it.

44 I discuss this issue in more detail in Section 1.6.1.

45 It is not my aim to provide an in-depth analysis of philosophical theories of the good life. For an overview of philosophical theories of the good life (or philosophical theories of well-being), see Hurka (2006) and Crisp (2008).
} 
judgments, without looking beyond the theory itself. In other words, a critic starts with a philosophical theory of the good life, which provides a specific view of the good life; in turn, digital media and digitally-mediated practices are assessed according to the view of the good life specified by the theory. Accordingly, a hedonist will assess digital media and digitally-mediated practices in terms of the pleasure and pain they produce. Likewise, a desire theorist will assess digital media and digitally-mediated practices with respect to the (new) desires they generate, as well as the desires they help satisfy. Finally, an objective list theorist will provide the list of goods that is constitutive of the good life, and digital media and digitallymediated practices then are assessed for their role in people's attainment of the goods on the list. ${ }^{46}$ In this line of thought, philosophical theories of the good life are the final arbitrator of normative judgements. Empirical data do not need to have any influence on the theory, and only serve to measure and determine whose life is good (or, at least, better) with respect to digital media and digitally-mediated practices. $^{47}$

\subsubsection{Putting the Good Life in Context}

If philosophical theorising alone is adequate for normative analysis of digital media and the good life, then only limited types of empirical data, e.g. the current and future development and use of digital media, its actual and predicted consequences, etc., are required. Other empirical data, such as actual discourses on digital media and the good life, are unnecessary. Recently, however, in other fields of normative research-notably in bioethics and normative political theory-empirical data including actual cases have been given a more prominent role in theorising. Empirical data are not merely instrumental for arriving at normative judgements; they are constitutive of normative judgments. Normative analysis of the good life in general and normative analysis of digital media and the good life in particular, I think, can benefit from an inclusion of actual cases in theorising, too. Here, I discuss Joseph Carens (2000, 2004)'s contextual approach. Particularly, I discuss the

46 This is, admittedly, an overtly simplified illustration. However, I think it suffices for illustrating the role of philosophical theories of the good life in normative analysis of digital media and digitally-mediated practices.

47 Ethicists who use of empirical data in this manner are characterised by Molewijk et al. as "the prescriptive applied ethicists". See Molewijk et al. (2004) for a useful typology for describing different relations between empirical research and normative ethics. Also, see Musschenga (2005). 
advantages of this approach and explain why it should be a welcome addition to the Walzerian approach.

Carens's inclusion of actual cases in theorising is not entirely novel. There have been various attempts to utilise empirical data (in a constitutive sense) in theorising, but, as Albert W. Musschenga (2005) points out, few of these attempts are accompanied by a serious methodological reflection. In this respect, Carens's contextual approach stands out from the other attempts by virtue of its explicit methodological guideline. Carens writes,

"A contextual approach [...] has five interrelated elements. First, it involves the use of examples to illustrate theoretical formulations. Second, it entails the normative exploration of actual cases where the fundamental concerns addressed by the theory are in play. Third, it leads theorists to pay attention to the question of whether their theoretical formulations are actually compatible with the normative positions they themselves take on particular issues. Fourth, it includes a search for cases that are especially challenging to the theorist's own theoretical position. Fifth, it promotes consideration of a wide range of cases, and especially a search for cases that are unfamiliar and illuminating because of their complexity." (Carens 2004, p. I I8)

I think the gist of Carens's methodological guideline is the second point, i.e. the use of actual cases in theorising (Carens 2004, p. 119). Using actual cases can clarify abstract theories, it can also prevent theorising from being "disconnected from reality". More importantly, it is the richness and complexity of real cases-which the hypothetical examples lack - that enable us to identify existing gaps between theorising and actual practices, as well as conflicts between normative judgements offered by theories and those prescribed by actual practices. In other words, the purpose of using actual cases is to direct our attention to the wisdom embedded in actual practices which theories fail to capture; and, in turn, to integrate it into the theory and our normative judgements. In this spirit, the contextual approach is indeed very similar to the method of reflective equilibrium, which aims to establish a normative foundation via mutual adjustments of normative theories and principles (e.g. moral theories, theories of the good life, etc.) and normative intuitions (e.g. moral intuitions, intuitions about the good life, etc.) to a state of coherence. $^{48}$

\footnotetext{
$4^{8}$ For an overview of the method of reflective equilibrium, see Daniels (2011).
} 
Yet, Carens's contextual approach differs from the method of reflective equilibrium significantly. For instance, their building blocks for normative reflection are clearly different: whereas the contextual approach starts with actual cases in normative reflection, the method of reflective equilibrium begins from theoretically-driven considered judgements. More importantly, although normative intuitions (and other type of empirical data, e.g. actual cases) may contribute to revisions of normative theories and principles in the method of reflective equilibrium, their contributions are only restricted to revisions of the theories and principles alone. This difference is significant because it entails normative intuitions and other types of empirical data can be disregarded once the theories and principles have been revised. So construed, normative judgements arrived at through the method of reflective equilibrium are ultimately based on normative theories and principles alone - albeit the fact that they may have been revised by empirical data. In other words, the method of reflective equilibrium does not take empirical data to be relevant in normative judgements as the contextual approach does. (For a quick comparison, see Figure 1 and Figure 2.)

Figure I.

The Method of Reflective Equilibrium:

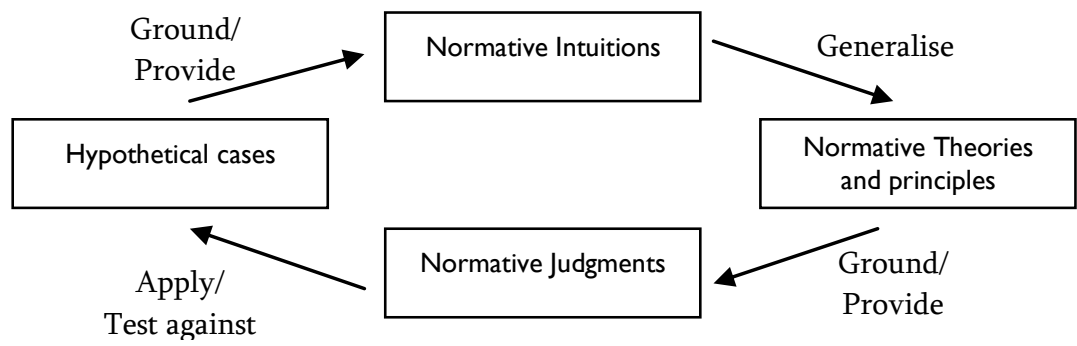

Figure 2.

Carens's Contextual Approach:

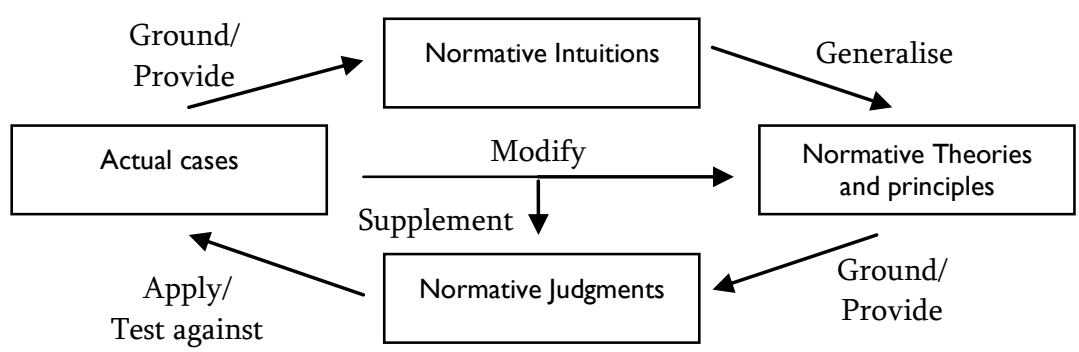


The use of actual cases (in a constitutive sense) in theorising allows the ensuing normative judgements to retain the wisdom embedded in practices, which is essential for operationalising normative judgements in the form of practical recommendations. If practical recommendations diverge too much from existing practices, their acceptability will be dubious (Birnbacher 1999). It is, therefore, important for a normative analysis of digital media and the good life to take seriously actual cases, as the normative analysis should be practically relevant as well. In addition, philosophical theories of the good life are based on a level of generality that does not immediately relate to the concrete issues that are specific to digital media and digitally-mediated practices. Inclusions of actual cases, therefore, become necessary to bridge the gap between abstract principles and values and their implementations. Finally, philosophical theories of the good life also leave open the issues that fall into the domain of permissible-but-not-required, which is precisely the case for many issues concerning digital media and digitally-mediated practices. ${ }^{49}$ For instance, philosophical theories of the good life will remain agnostic about the use of a particular type of digital media if the use makes the same contribution to the good life at a theoretical level. On these issues, it is up to the local circumstances for determining which option is better. Hence, actual cases are indispensable in a truly normative analysis, i.e. a normative analysis that is actionguiding, because they provide insight into the local circumstances that are unavailable to pure philosophical theorising.

\subsubsection{Indispensability of Prudential Appraisals}

I shall now illustrate how prudential appraisals can supplement philosophical theories of the good life in more detail. It should be remembered, however, that there are two different projects in the philosophical research on the good life. There is, on the one hand, the descriptive project, which aims to provide a conceptual analysis of the concept of the good life itself. On the other hand, there is the normative project, which aims to formulate a theory of the good life that is actionguiding. It is within the normative project, I think, philosophical theories can benefit most from an inclusion of actual cases. Here, I show that philosophical

49 Levy (2007) has made a similar point concerning theories of justice and the questions associated with multiculturalism. Briefly, he notes that various issues, e.g. state symbolism, institutional design, etc., cannot be answered by (philosophical) theories of justice. They have to be settled by looking at the local circumstances. 
theories of the good life will be truly action-guiding only if they include prudential appraisals in the course of normative reflection.

The importance of prudential appraisals, I believe, is relatively clear for objective list theories. Such theories, as I have noted, provide a list of the goods that are said to be constitutive of people's good lives. Yet, an objective list theory, aiming to be universal, will remain abstract, general and idealised. As such, the nature of objective list theory immediately raises questions about its connection to reality. In particular, as many researchers have convincingly argued, the development and use of digital media have radically transformed society, and an objective list theory that ignores social, cultural and political changes will fail to capture the new issues that arise from the changes. Actual cases, therefore, can inform the objective list theory on these changes and enable it to take them into consideration in theorising. Here, prudential appraisals, which reflect the values shared by (at least a specific group of) people in public, also provide the shared views of the good life. Hence, employing prudential appraisals in normative reflection will prevent the list of the goods in the theory from becoming drastically disconnected from existing practices. At the same time, the normative and evaluative corpus in prudential appraisals also provides a necessary resource for translation of the goods on the list into acceptable practical recommendations. As such, through prudential appraisals, the list of the goods in an objective list theory can be turned into practical recommendations. In cases where the practical recommendations derived from the list of goods in an objective list theory come into conflict with the prudential appraisals, adjustments of the practical recommendations (and, perhaps, the list of goods) have to be made by reflecting on the prudential appraisals. In short, prudential appraisals help to formulate and realise the practical recommendations based on an objective list theory, and thereby allow an objective list theory to be action-guiding in a realistic sense.

For hedonists and desire theorists, however, it may not be immediately clear why prudential appraisals matter at all. Prudential appraisals, in my account, discuss what digital media and digitally-mediated practices are good for a person. However, if either hedonism or desire theory is true, then the goodness-for in the prudential appraisals will be a subjective matter. So construed, one person's prudential appraisal may be completely irrelevant to another person, because what is pleasurable or desirable to one may not be the same as what is pleasurable and desirable to another. This is so because something is pleasurable for a person only if he feels the pleasure and his desire is satisfied only if it is genuinely the person's own. In this sense, for hedonists and desire theorists, prudential appraisals may be 
reduced to idiosyncratic opinions of what digital media and digitally-mediated practices are good for only for a particular individual. ${ }^{50}$ Yet, I want to argue that even if prudential appraisals are similar to subjective opinions, they are still needed if the hedonists and desire theorists are to offer practical recommendations.

Hedonism and desire theories, in whichever formulation, share the problems faced by objective list theories in that they are abstract, general and idealised. Hence, in a similar manner, prudential appraisals allow hedonists and desire theorists to formulate practical recommendations that can be acceptable to the public by stressing their continuity with existing practices. Here, the role of prudential appraisals is essentially the same in hedonism and desire theories as in objective list theories. However, hedonism and desire theories face a greater challenge in terms of action-guidingness because they do not by themselves provide any resources for constructing practical recommendations. Hedonism and desire theories are formal theories, i.e. they only specify the formal condition(s) of the good life. Accordingly, the good life is either a pleasurable (and painless) life or a life in which most (or all) desires get satisfied. However, the formal condition(s) does not, and cannot, help formulate practical recommendations on concrete issues. In order to formulate practical recommendations, critics need to know why and how people think digital media and digitally-mediated practices make them happy or satisfy their desires. In this respect, prudential appraisals provide the normative and evaluative corpus for hedonists and desire theorists to identify the appropriate and plausible reasons for urging one option over another. In other words, prudential appraisals can supplement hedonism and desire theories with the information required to formulate practical recommendations, even if they appear merely as subjective opinions. ${ }^{51}$

\footnotetext{
${ }^{50}$ It is also possible to criticise the understanding of prudential appraisals as such by invoking the communitarian idea of the self, e.g. those who argue in this line can point out that people's decisions cannot be entirely separated from their community. Hence, they will inevitably be influenced by their community's normative and evaluative standards. As a result, prudential appraisals cannot simply be idiosyncratic opinions in the radical sense. I think this objection is plausible, but I will not explore this option here. ${ }^{51}$ Here, I present the problem for hedonism and desire theories in terms of the formulation of practical recommendations. However, the problem equally applies to any circumstances where a critic needs to rationally defend his practical recommendations because the formal condition(s) specified by hedonism and desire theories offer no resource to justify particular practical recommendations as well. See P.-H. Wong (2010).
} 
In summary, philosophical theories of the good life may still play a role in grounding normative judgements concerning the relations between digital media and the good life. However, they alone are insufficient in terms of actionguidingness, and they are also insufficient for researchers to offer practical recommendations. A truly normative analysis of digital media and the good life, therefore, have to acknowledge the place of prudential appraisals, which is indispensable in formulations and realisations of practical recommendations. And, this - is the conclusion of my additional argument.

\subsection{Conclusion}

In this chapter, I have developed and elaborated an approach for the present study, i.e. the Walzerian approach. This approach takes prudential appraisals seriously in that it seeks to examine the existing views of the good life in prudential appraisals and to use the normative and evaluative resources made available by a reflection of them. Normative analysis of digital media and the good life, as I have argued, requires existing views of the good life because our normative judgements pertaining to the good life are based on our practical identity and the ideals it embodies, which, in turn, are socially and culturally specific. As prudential appraisals reflect the practical identity and the embodied ideals shared by the critics and the people, they serve as a useful window into people's practical identity and the embodied ideals, and they also provide the normative and evaluative resources for offering practical recommendations that allows a normative analysis of digital media and the good life action-guiding. Finally, I have offered an additional argument for the inadequacy of philosophical theories of the good life for normative analysis of digital media and the good life.

In short, there are two main objectives in this chapter. Firstly, it aims to illustrate the role of actual discourse (i.e. prudential appraisals) in normative analysis of digital media and the good life. Secondly, it also aims to show that normative analysis of the relations between digital media and the good life must reserve a place for our socially and culturally based practical identity and the ideals it embodies, because they are the grounds for our normative judgements on the relations between digital media and the good life. 



\section{CHAPTER TWO}

Modern Identity and the Good Life A Philosophical and Empirical Analysis

I have argued in the previous chapter that our practical identity is a mode of selfinterpretation and self-understanding, which embodies certain ideals. The embodied ideals, in turn, offer us specific views of the good life. Based on the person we are and the person we should be, practical identity offers us a vision of how we should live. To use Charles Taylor's terms, the ideals embodied in practical identity are the basis of "strong evaluation", i.e. "the discrimination of right or wrong, better or worse, higher or lower, which are not rendered valid by our own desires, inclinations, or choices, but rather stand independent of these and offer standards by which they can be judged" (Taylor 1989, p. 4). As practical identity provides us the standards for normative judgements, to fully understand normative judgements on digital media and digitally-mediated practices in our time, the ideals of modern identity must first be unpacked.

Modern identity ${ }^{1}$, which permeates Western cultures in the present day, has its own view(s) of the good life. In this chapter, I will explicate them through

\footnotetext{
Following the common usage of the terms 'modernity' and 'modern identity', I use them to refer to the contemporary Western culture, i.e. European and North American cultures, and the notion(s) of the self in the West. See, e.g. Taylor (1989), Giddens (1990,
} 
examining the identity developed since the Enlightenment. The mode of selfinterpretation and self-understanding developed from the Enlightenment, as many researchers have observed, forms the basis of self-interpretation and selfunderstanding of the present day. ${ }^{2}$ Yet, as Anthony Giddens has shown, modern identity (or the modern self) is not merely passive, i.e. "the new mechanisms of selfidentity which are shaped by-yet also shape-the institutions of modernity"; therefore, it will not be enough merely to study modern identity in isolation from the institutions of modernity (Giddens 1991, p. 2). Since modern identity and the institutions of modernity are mutually shaping each other, in my discussion of modern identity, I will connect it to the institutions of modernity whenever it is necessary.

I proceed by first explaining the relations between modernity and modern identity in Section 2.1. Different dimensions of modernity have been extensively studied by various disciplines. For my discussion of modern identity, the cultural and epistemological dimension of modernity is particularly relevant. And, Taylor's Sources of the Self (1989) is arguably the most comprehensive account of the cultural and epistemological dimension of modernity currently available. Hence, my discussion of modern identity begins with Taylor's account of modern identity in Section 2.2. Despite its comprehensiveness, Taylor's account is inadequate to account for modern identity in its present day form as modernity has currently reached its second stage. Since Taylor's account is undivided, i.e. it does not distinguish various stages of modernity, it is insufficient to capture the distinctive features of modern identity in the new stage of modernity. So, in Section 2.3, I turn to the works of Anthony Giddens and Ulrich Beck, which focus on the latest form of modern identity. After identifying the key features of the different notions of the self in modern and late modern societies, I explicate the views of the good life explicitly and implicitly embodied in them in Section 2.4. In this section, I also examine the empirical research which lends support to my characterisation of the views of the good life in modernity and late modernity.

1991), Beck (1992), and Beck, Giddens \& Lash (1994). Hence, my use of 'modernity' and 'modern identity' simply refers to Western modernity or modern identity in the West.

2 For example, see Taylor (1989), Dupre (2004) and Seigel (2005). 


\subsection{Modernity and Modern Identity}

Previously, I have argued that (self-)identity is derived from the wider sociocultural context. In this sense, modern identity is inherently dependent on modernity because modernity has its unique social, cultural and institutional dimensions, which provide the basis for the formation and understanding of the (modern) self. So construed, a study of modernity naturally serves as an anchor for any inquiry into the modern self. Hence, it is helpful to begin my discussion with a brief survey of theories of modernity.

Different theories of modernity have been developed in various disciplines. Following Philip Brey, I distinguish two types of theories of modernity, i.e. cultural and epistemological theories and institutional theories. Cultural and epistemological theories "focus on the distinction between pre-modern and modern cultural forms and modes of knowledge", whereas the institutional theories "focus on the social and institutional structure of modern societies" (Brey 2003, pp. 36-37). Cultural and epistemological theories, as Brey summarises, focus on the transformation of the concept of the self (or the concept of the subject), the worldview and the methods of and the concepts in scientific inquiry, etc. from traditional culture to modern culture. In addition, they usually view the Enlightenment as the mark of the rise of modernity. While cultural and epistemological theories are interested in the transformation of cultural and epistemic concepts, institutional theories are interested in the institutional structures of modern societies and how they differ from traditional societies. Particularly, most institutional theories attempt to establish causal explanations from the changing social, political and economic conditions to the transformation of the related institutional structures. Hence, most institutional theories can also be seen as theories of modernisation, which aim to examine "the process of large-scale change through which a certain society tends to acquire the economic, political, social and cultural characteristics considered typical of modernity" (Martinelli 2005, p. 5).

Although these two types of theories approach modernity with a different emphasis, it will be a mistake to take them to be completely separated from one another, as the possibility of cultural and epistemological transformation depends essentially on transformation in a society's institutional structures. However, it would equally be a mistake to assimilate them into one grand theory of modernity. To see why, it is useful to use Taylor's (1999) distinction of cultural theories of modernity and acultural theories of modernity. As Taylor notes, cultural theories of modernity study modernity in terms of a rise of a new culture from its predecessors, and acultural theories of modernity study modernity "in terms of some cultural- 
neutral operation, which is not defined in terms of specific cultures it carries us from and to, but is rather seen as of a type that any traditional culture could undergo" (Taylor 1999, pp. 154-155). Accordingly, an attempt to encapsulate the cultural and epistemological transformation of modernity in an expanded institutional theory of modernity can be viewed as an instance of acultural theory. The danger of assimilating the two types of theories in this manner is precisely an ignorance of the contributions and the determents from distinct social and cultural heritages. For my study of (modern) identity, this danger is especially acute: ignoring social and cultural heritages means that there is, ultimately, only one form of (modern) identity, which in later chapters, I will show, is mistaken.

My goal here is to specify the ideals (or the views of the good life) embodied in the modern self. The first step towards this goal is to identify the defining features of the modern self. In order to do so, I examine the shift of cultural and epistemological concepts that give rise to it. As Brey points out, the analysis of the concept of the self (and the related cultural and epistemological concepts) is primarily a task for cultural and epistemological theories of modernity. Hence, I limit my study to the existing cultural and epistemological theories of modernity. However, as Giddens also points out, it is a peculiar feature of self-identity in late modern societies that it actively shapes the institutions of (late) modernity while at the same time being shaped by them. So, I shall refer to institutional theories in my discussion of self-identity in late modernity. It is important, as self-identity is shaped by the institutions of (late) modernity, without taking the changes in the institutional dimension into account, the discussion of the modern self will necessarily be incomplete.

Although the concept of modernity figures importantly in the study of modern identity, I shall not enter into the debate on the nature of modernity. Discussing the nature of modernity is well beyond the scope of this chapter; my aim is only to offer an account of the modern self which can help us to understand the views of the good life in the present day, and I shall fulfil this aim by means of a careful reading of the existing works on modern identity from prominent researchers on the topic. I first examine Taylor's account of the modern self in Sources of the Self, as it is perhaps the most comprehensive philosophical study of the modern self currently available. Despite its comprehensiveness, however, it has presumed an undivided concept of modernity, which fails to account for the distinctive features in the new stage of modernity. Turning to more recent works on modern identity by Giddens and Beck et al., I examine the nature of self-identity in late modern societies. Nonetheless, Taylor's account remains relevant, for the ideals in the modern self are 
not being abandoned entirely in late modernity, i.e. while self-identity in late modern societies has its distinctive features, it still has strong affinities with the modern self in Taylor's account.

\subsection{Taylor on Modern Identity}

In Sources of the Self, Taylor provides an insightful account of modern identity. 'Modern identity' refers to the uniquely modern ways of interpreting and understanding the self; and, what distinguishes modern identity from other modes of self-interpretation and self-understanding are (i) the modern inwardness, (ii) the affirmation of ordinary life, and (iii) the expressivist notion of nature as an inner moral source (Taylor 1989, p. x). The modern inwardness is "the sense of ourselves as beings with inner depths, and the connected notion that we are selves" (Taylor 1989, p. x). Such inwardness, Taylor thinks, is best exemplified by our selfinterpretation and self-understanding through distinct locales of inner and outer and the opposition of "inside-outside" (Taylor 1989, p. 111). For the affirmation of ordinary life, Taylor refers to a shift in the locus of importance in human life: higher, spiritual activities are being replaced by ordinary, mundane activities, such as labour, marriage and family, as the primary preoccupation in people's pursuit of a meaningful life (Taylor 1989, p. 211, 212-213). Finally, by "expressivism”, Taylor means the realisation of one's nature by "articulating what one finds within" (Taylor 1989, p. 374); and, the expressivist notion of nature as an inner moral source entails that self-realisation through articulating or expressing one's inner nature become morally significant in modernity. Taylor notes that these features are unique in modernity such that the modern self, which manifests these features, is so different from other construals of selfhood.

However, by identifying the three basic features that are unique to the modern self, Taylor does not mean to offer a unitary account of the modern self. Through a historical narrative, he has shown how these basic features resisted and intertwined with each other and gradually brought different strands of the modern self into being. For Taylor, to fully understand the modern self, a one-dimensional account has to be replaced by an account which respects the multiplicity and complexity of the modern self. Underlying Taylor's use of historical narrative and interpretative explanation is the assumption that the self cannot be adequately understood by any accounts which isolate the self from its historical context, because the self has emerged from and developed through a dialectical process that refers back to the 
historical context of which it permeates. A new notion of the self has to be built on its predecessors either by supplementing them, rejecting them or a mix of both. ${ }^{3}$

If the modern self has to be understood dialectically, then the original notion(s) of the self of which the modern self is built on must first be identified. Taylor suggests that the original notion came from Christianity, which is based on the Christian God. Accordingly, different strands of the modern self are products of dialectical processes against the Christian, theistic mode of selfhood. Taylor also notes that the theistic mode of selfhood, while already transformed from its ancient predecessors, still exerts enormous influence in modernity; and, particularly important is its core tenet of (Christian) God as the ultimate source of meaning (i.e. the good, the right and justice) still enjoys an unquestionable status (for the believers) today. Unlike the theistic mode of selfhood, the two strands of the modern self are always being questioned (Taylor 1989, pp. 317-318). The first strand of the modern self can be labelled as 'the disengaged self', the root of which arguably can be traced to Descartes. And, the second strand of the modern self can be labelled 'the expressive self', which has Rousseau and the romantics as its major interlocutors. In this section, I shall elaborate Taylor's discussion of the two strands of the modern self. In particular, I shall focus on the key features of the two strands of the modern self and explain how those features have become integral to them. Since they are shaped by the dominant ontology and epistemology of the time, it is crucial to the discussion to unpack the paradigmatic shift in ontology and epistemology which has provided the grounds for the transformation of the self.

\subsubsection{The Disengaged Self}

The disengaged self, as Taylor unfolds, is characterised by the disengagement stance, the primacy of instrumental reason and an accompanied procedural conception of reason. This strand of the modern self portrays its ideal as an autonomous, rational being, who is self-determining and self-responsible, and whose acts ought to be determined by reason and reason alone. The formulation of the subjects as such places (human) dignity at the centre of the disengaged self. To

\footnotetext{
3 Taylor's 'dialectical' account of the (modern) self is both diachronic and synchronic. Diachronically, the development of the modern self is portrayed by Taylor in a Hegelian spirit. In his historical narrative, a concept of the self supplements or replaces another by building on its predecessors. At the same time, Taylor's 'dialectical' account is also synchronic with respect to the emergence of the modern self, i.e. the self comes into being by interacting and communicating with the others within one's community.
} 
fully appreciate how the disengaged self has become seemingly an indispensable way to interpret and understand the self in modernity, it is necessary to identify the major shift in ontology and epistemology in early modernity, which has opened up the conceptual space for a new notion of the self and made the disengaged self creditable.

Disengagement can be understood both as an epistemological and as a moral perspective. As an epistemological perspective, disengagement requires the subjects to take an object of inquiry as something to be intrinsically distinct from them. ${ }^{4}$ Similarly, as a moral perspective, disengagement requires the moral subjects to be distinct from and prior to moral norms and principles; moral norms and principles must be the things conferred by the moral subjects. ${ }^{5}$ The primacy of instrumental reason refers to a specific understanding of the purpose of reason, i.e. reason as rational control. In this sense, reason (re)defines the relations between the subjects and their surroundings, and the things around the subjects now become raw materials to be altered and used for their purposes. Finally, the procedural conception of reason means that rationality is to be defined "in terms of the standards by which we construct orders in science and life" (Taylor 1989, p. 156). In other words, reasoning is now an activity which aims to generate specific outputs according to specific sets of (constructed) rules; it also means that the ultimate goal of reason(ing) has shifted from the quest for substantive meaningfulness to the act of thinking itself.

Taylor argues that the emergence of these features can be explained by a different understanding of "rule by reason", which he calls internalisation, "a replacement of [the] understanding of the dominance of reason, [as rule by rational vision of order] by another, [...] in which the order involved in the paramountcy of reason is made, not found" (Taylor 1989, p. 124). It was brought about by a paradigmatic shift in both ontology and epistemology peaked at the Enlightenment. Indeed, the very possibility of internalisation hinges on a break away from the rational vision of order. In this sense, the mechanistic picture of the universe inaugurated by Galileo, and subsequently picked up and extended by Descartes and

\footnotetext{
4 For example, Descartes's cogito sought to understand the foundation of knowledge via the mind-body distinction, in which the body is intrinsically distinct from the mind, which is where the subjects believed to be.

5 For example, in Kantian ethics, the moral subjects are essentially distinct from and prior to any given (substantive) moral norms and principles. It is only through the subjects' own rational scrutiny, can it become morally relevant.
} 
Locke, has provided the ultimate ground for internalisation. In the mechanistic picture of the universe, the universe is to be viewed as an impersonal entity functioning like a machine. So construed, the universe could no longer be taken to be embedding and providing any meaningful orders, as Taylor puts it, the universe has become "expressively dead" (Taylor 1989, p. 148). Later, this mechanistic picture would be extended from an understanding of the universe to other aspects of human affairs as well. Accompanied by the ontological shift was an epistemological shift. The mechanistic picture of the universe requires the subjects to see the world as something distinct from and external to them. In this new epistemology, the universe becomes a lifeless object of inquiry, of which meaning is not, and cannot be, found but only conferred by the subjects. In other words, knowledge is not consisted in the universe, but it is accurate representations of the universe within the subjects' minds. Moreover, the success of the Scientific Revolution had further credited (natural) sciences as the only legitimate route to knowledge.

Given the mechanistic picture of the universe, and, subsequently the mechanistic theories of the human mind, as well as similar mechanistic views of culture and society, and together with the new notion of knowledge which aims at providing causal-scientific explanation, it should be obvious how internalisation and the related features become essential to the disengaged self. For instance, internalisation requires a break away from the rational vision of order, which becomes possible and plausible when the mechanistic picture of the universe is incapable of issuing meaningful orders to the subjects. Also, the subject-object distinction arises from the new ontology and epistemology, of which the subjects now assume the role of knowers, whose task is to construct accurate representations of an external reality. As a result, reason(ing) changes from a form of attunement that sought after the meaningful order(s) embedded in and provided by the universe to a "capacity we have to construct orders which meet the standards demanded by knowledge, or understanding, or certainty" (Taylor 1989, p. 147). In other words, the power to issue meaningful orders is stripped away from the universe and crown on the subjects' minds.

In a similar vein, we can explain the three features of the disengaged self with the ontological and epistemological shift, too. The new notion of knowledge required the subjects to represent the external reality with clarity and distinctness. And, clarity and distinctness, it is believed, can only be attained by taking up a third-person standpoint. In other words, the subjects have to disengage themselves from objects of inquiry if they are to acquire any knowledge of them. Similarly, the 
primacy of instrumental reason can be explained by referring to the new ontology and epistemology, as they prioritised the subjects over the objects. Also, as the subjects' surroundings became meaningless, they are viewed as raw materials for the subjects. In this strand of the modern self, therefore, the notion of knowledge has increasingly emphasised knowledge as a means to control. Finally, in an organic picture of the universe, the purpose of human beings is specified by and within the rational vision of order of the universe; and, the ideal of human beings is already (pre)defined by their telos. In this picture of the universe, reason is to attune human beings to the universe and to enable them to fulfil their telos. The mechanistic universe does not mesh well with a teleological view of human nature. No substantive norms and values can be derived from the mechanistic universe, and without any substantive norms and values in the external reality, reason turns to focus on formal rules and procedures that can provide acceptable justification for the norms and values, which, in turn, explains the rise of the procedural conception of reason.

In short, the disengaged self puts the subjects at the core and frees them from any pre-given nature. What emerges, then, is a subject that determines its own course of action via their own norms and principles and through their own rational reflections. In its extreme, this is what Taylor calls a "Lockean punctual self": a subject which takes a radical stance of disengagement to himself with a view to remaking, i.e. adopting the third-person standpoint in their self-interpretation and self-understanding, thus to transforming the self into an object of inquiry, and thereby seeing the real self as "extensionless" or, simply, as the power to fix things as objects (Taylor 1989, pp. 171-172). Accordingly, the ideal of the punctual self is someone who is "able to remake himself by methodical and disciplined action" (Taylor 1989, p. 159). Here, autonomy becomes essential, as the subject is deemed as responsible to reflect, to choose and to act, and what constitutes the subject's responsibility is exactly the freedom of which the subject enjoys. The disengaged self, therefore, presupposes autonomy. ${ }^{6}$ Also important in the disengaged self is the idea of human dignity: human beings are valuable precisely because they are autonomous and rational, as they have the power to reflect, to choose, and to act; and, they are also self-determining in that it is only through their rational scrutiny

\footnotetext{
6 This is best exemplified by Kantian ethics, in which morality is to be defined in terms of autonomy, i.e. permissibility of an act is determined not by any (imposed) norms and principles or specific outcomes, but by a subject's rational scrutiny in terms of his reflective (self-)acceptance of moral norms and principles.
} 
they act according to moral norms and principles; as Taylor quotes Kant, "[e]verything in nature works according to laws. Only a rational being has the capacity of acting according to the conception of laws, i.e. according to principles" (Taylor 1989, pp. 364-365). In sum, the disengaged self ushers an ethical outlook that demands self-responsibility and self-discipline based on rational scrutiny.

\subsubsection{The Expressive Self}

In many ways, the second strand of the modern self, i.e. the expressive self, can be seen as a reaction against the rise of the disengaged self. As we have discussed, the disengaged self places (instrumental) rationality at the core of its ideal. Against such an ideal, the expressive self rejects the disengaged self's lone emphasis on (procedural) reason, and embraces a broader notion of nature as inner voice, which takes seriously one's feeling as a guide to their ideals. In Taylor's view, the expressive self is captured by Romanticism's doctrines, i.e. the rights of the individual, the power to creative imagination and the importance of feeling for the search of a meaningful life (Taylor 1989, p. 368). These doctrines are supported by a different view of human nature defended by the romantics. To understand the expressive self, therefore, it is necessary to find clues from the view of human nature defended by the romantics.

Unlike the ideal of the disengaged self, which can be defined exhaustively by a subject's rational nature, and thus individual differences "are only unimportant variations within the same basic human nature" (Taylor 1989, p. 375), the expressive self takes individual differences, i.e. individuality, seriously. For the expressive self, individual differences "entail that each of us has an original path which we ought to tread; they lay the obligation on each of us to live up to our originality" (Taylor 1989, p. 375). The emphasis on individuality as such is linked to the view that the ideal embodied in the expressive self is to be attained by following the inner voice and the belief of the importance of creativity for attaining such an ideal. Also, implicit in the emphasis on individuality is the assumption that individuals are different in their very nature; and, if originality is the goal to strive for, then what is required is to look at the very sources of individual differences and to promote them. For the expressive self, individual differences lie precisely in our nature. In this way, as Taylor argues, "we come to define what nature is as a source in the course of articulating what it inclines us to" and, when it is so defined, "the articulation must be partly in terms of sentiment" (Taylor 1989, p. 373). So construed, the role of creative imagination in the expressive self has also become obvious: given the emphasis on individuality and originality, as well as the related 
notion of nature as an inner source, it is only through articulating what we find within, we come to know what our nature is. In Taylor's terms, "the realisation [of our nature] in each of us is also a form of expression... To express something is to make it manifest in a given medium" (Taylor 1989, p. 374). Yet, such a making is to be understood as "a making, a bringing of something to be". It requires, in other words, our creative imagination.

The expressive self relies on a view of human nature that is different from the one in the disengaged self. As the true human nature for the disengaged self is reason (and reason alone), the role of nature in the disengaged self is rendered minimal. The expressive self, on the other hand, reconnects the self with a broader notion of nature, which also accommodates human sensibility. Behind this broader notion of nature, it is the Rousseauian view of human nature, according to which human depravity is a real possibility and it cannot be avoided or escaped by an increase in practical knowledge (Taylor 1989, p. 356). The Rousseauian view of human nature, in effect, has led the expressive self to an opposite direction to the disengaged self: it implies that the ideal embodied in the expressive self cannot be aligned with reason. As Rousseau and his followers substitute reason with our inner nature, they also reject the disengagement stance. Since the ideal of the expressive self is derived from our inner nature, to attain this ideal, the subjects need to turn inwards and towards their own nature. Instead of disengaging themselves from their nature, the subjects must reengage themselves with their nature and (re)discover what their unique nature consists of. The rejection of reason as a path to attain meaning calls for an alternative, and the expressive self finds such an alternative in our inner feeling. For Rousseau and the romantics, attaining meaningfulness is a matter of listening to our inner voices. Hence, the ethical outlook of the expressive self differs drastically from the one in the disengaged self. Its ultimate goal is about self-discovery and self-realisation, and thus can also be viewed as an ethics of authenticity: an ethics that aims at being true to oneself. At its extreme, it blends ethics and aesthetics together and becomes radical subjectivisation.

\subsubsection{The Affirmation of Ordinary Life}

Despite the incompatibility of the disengaged self and the expressive self, both strands of the modern self share the view that a subject is not to be defined by something (pre-)given. For the disengaged self, the self is determined solely by the subject's rational scrutiny, as for the expressive self, it is the subject's unique inner voice that determines the self. Both strands of the modern self, therefore, place the 
subject, i.e. the self, at their core. Besides the subject-centeredness, there is also a fundamental shift in the nature of their ideals: the ideals embodied in the modern self are no longer defined in terms of higher, spiritual activities but in terms of other worldly activities; and, the replacement of the locus of importance in human life is what Taylor calls "the affirmation of ordinary life" (Taylor 1989, p. 211, 212213).

The replacement, as Taylor has shown, began from the Reformation, during which the notion of mediation (and the related ideas) was rejected by the Protestant reformers (Taylor 1989, pp. 215-218). According to the notion of mediation, the church is the bridge between people and God. So construed, salvation is possible only if people participate in the church's life. The notion of mediation, therefore, imbued a special status to activities organised by the church. By rejecting mediation, participation in the church lost its meaning, i.e. activities organised by the church no longer holds the key to people's salvation; or, to put it in another way, participation in the church's life provides no better chance for salvation than any other activities. Hence, the rejection of mediation has provided an opportunity for the re-valuation of ordinary, mundane activities. Also implicit in the notion of mediation is the idea that certain activities, e.g. higher, spiritual activities, are more valuable than others. As the supposedly more valuable spiritual activities and mundane activities come to be on equal standing, the value of people's lives can no longer be defined and determined by the kinds of activities he is involved in, because these activities do not distinguish themselves by their contribution towards a meaningful life. In short, what counts towards a meaningful life "turns on the spirit in which one lives whatever one lives, even the most mundane existence" (Taylor 1989, p. 224).

The re-valuation of ordinary life is accompanied by a new notion of calling, too. Serving God is no longer only confined to spiritual activities, and the duties to God can now also be fulfilled by mundane activities in people's ordinary lives. So, people's spirituality is to be measured by their dedication to the activities they are engaged in, regardless of what kinds of activities they are. In other words, people are "to serve God in serving of men in the works of our callings" (Taylor 1989, Joseph Hall, quoted p. 225). This new notion of calling, as Taylor observes, provided the foundation of what Weber calls the "bourgeois ethics", which stresses the importance of productivity and universal beneficence. From the affirmation of ordinary life and the shift towards bourgeois ethics, it is not difficult to discern that the philosophical foundation of utilitarianism was in the making. Utilitarianism, ultimately, rests on two basic doctrines: (i) efficiency based on the calculation 
model and (ii) impartiality. Both of the doctrines are supported by the new notion of calling via its emphasis on industriousness, instrumental rationality and universal beneficence. With the affirmation of ordinary life, and in particular with an increasing emphasis on the avoidance of suffering, happiness is now being placed at the centre of moral consideration. The combination of these ideas naturally leads to an ethical position characterised by a maximisation of happiness for all (Taylor 1989, pp. 238-240).

Another important consequence of the affirmation of ordinary life is that it set the ground for modern non-belief, as it has moved people away from a hierarchical order of the spiritual and the ordinary, and has made human beings central to moral consideration. But, modern non-belief will not be possible unless alternatives are made available (Taylor 1989, pp. 309-314). Here, Taylor helpfully notes that the development of Deism has provided the subsequent ground for non-believing. As Deism aligned God's laws with natural laws, to live by God's plan is to live by nature, i.e. nature is simply designed by God. In the Deistic picture, God is subsumed under reason (or, God is equivalent to reason). Accordingly, God cannot but act according to reason because reason is constitutive of God. This relation, supplemented by the view that natural laws can be understood through human rationality, effectively erased the mystery of religion and the idea of grace in Christianity. They appear to be redundant if humankind can understand God's plan by reason(ing). Perhaps, more importantly, it is that God's power is superseded by the plan of God (Taylor 1989, p. 271). The rise of a Deistic account of natural laws, together with an increasing confidence in the explanatory power of natural sciences, eventually marked the secularisation of modernity.

Taylor's discussion of the disengaged self, the expressive self and the affirmation of ordinary life gives us a comprehensive view of the basis of modern identity. In my presentation of Taylor's account, it may seem as though the two strands of the modern self, as well as the theistic mode of selfhood, are completely separated from one another. However, we should resist this impression. As the title of Sources of the Self has already hinted, Taylor aims to discuss the sources of modern identity. These sources, as Taylor has painstakingly reminded us, constantly interact with one another in our self-interpretation and self-understanding. As the ideals embodied in them are not always compatible, we can discern tensions about various values in modernity (see, e.g. Taylor 1989, Chapter 25). In fact, these tensions about values signify a further aspect of modernity, namely the plurality of values. In short, the ideal of the disengaged self and of the expressive self, the affirmation of 
ordinary life, and the plurality of values have summarised Taylor's view of modernity and the modern self. It should be clear by now, in Taylor's view, the modern self is uniquely modern because of these features.

\subsection{Late Modern Societies and Self-Identity: Giddens and Beck et al.}

While Taylor's account provides us important insight into modern identity by revealing its sources, i.e. the disengaged self, the expressive self and the remnant of theistic understanding of selfhood, his discussion remains very much Enlightenment-based. Yet, as many theorists of (post)modernity have noted, modernity has moved on to another stage even if it is not the case that, as some postmodernists claim, modernity has come to an end. They point to the fall of the Soviet Union, the rise of post-industrialism, the coming of the information technology revolution, the intensification of globalisation, and other major events, as well as the main social, political and economic changes that happened in the late 20th century as indicators of a new epoch. Whatever events and changes best signify the new stage of modernity, it is generally agreed that the institutions of modernity are different in the new epoch. Since our self-identity depends on the wider social and cultural context, it means that Taylor's account of modern identity, which does not include recent historical events and social, cultural and political changes, is insufficient to account for modern identity in the present day. Here, I examine the works by Giddens and Beck, which are devoted to investigating the mechanisms of identity construction in the present day. Since their views on selfidentity are derived from their accounts of the new stage of modernity, I shall start with their discussion of (late) modernity first.

\subsubsection{From Modernity to Late Modernity}

In Giddens's view, modern societies are discontinued from traditional societies most significantly by the intrinsic dynamism and the globalising nature of modern institutions (Giddens 1990, p. 6). He characterises modern societies by four institutional dimensions: capitalism, industrialism, the apparatus of surveillance, and the monopoly of the means of violence. These four institutional dimensions are interrelated, and they go hand-in-hand with the development of nation-states, and the nation-state system, in modernity (Giddens 1990, pp. 55-63). According to Giddens (1990, Chapter 2), the dynamism and expansionist tendency are fuelled by:

(i) The separation of time and space. This refers to the condition in which 
time is emptied from space and space is dislocated from place. Time and space are re-organised by means of precise temporal and spatial zoning. The separation of time and space not only allows the connection of the local and the global, it also creates an objective 'history' by situating historical events in the standardised temporal and spatial zones, Moreover, it provides the prime conditions for the process of disembedding

(ii) Disembedding refers to the development of abstract systems, including symbolic tokens such as money and expert systems such as professional expertise, which "lift out" social relations from local contexts of interaction and restructure them across indefinite time and space.

(iii) The reflexive appropriation of knowledge refers to the production of systematic knowledge about social life and the individual's reflective and reflexive adoption of knowledge in structuring their life; reflexivity as such, in turn, undermines the fixities of tradition.

The late modern age is, therefore, a radicalised modernity, in which "the consequences of modernity are becoming more radicalised and universalised than before" (Giddens 1990, p. 3). In other words, dynamism and the globalising tendency are driven to the extreme by these processes.

Similarly, Beck describes the transition to the second stage of modernity as a transition from an "industrial" or "class society" to a "risk society" (Beck 1992). ${ }^{7}$ In their more recent researches, Beck and his colleagues (Beck et al. 2003, Beck \& Lau 2005) have attempted to capture the second stage of modernity by a more comprehensive research programme of "second modernity", in which they attempt to understand how the first modernity, which is based on (i) a 'contained' model of nation-state societies, (ii) a programmatic individualisation which an individual's freedom and equality are still moulded by social institutions, (iii) a work society that emphasise full employment, (iv) a concept of nature founded on its exploitation, (v) a scientifically defined concept of rationality and (vi) the principle of functional differentiation with respect to their development, is influenced by processes such as globalisation, intensification of individualisation, the gender revolution, new models of employment, and global risks (Beck et al. 2003, pp. 3-8; Beck \& Lau 2005). And, the impacts of these processes are, as they argue, that the fundamental assumptions of the first modernity, i.e. controllability, certainty and security, are

7 Also, see Beck, Giddens \& Lash (1994). 
being given up. Hence, the second modernity amounts not only to new institutions, but also to the very organising principles of (modern) society as well.

Both Giddens and Beck have pointed out the transition from the first stage of modernity to the second stage has significantly affected the mechanisms of identity construction; and, as I shall demonstrate, the different mechanisms also reflect a different notion of the self. Their views on self-identity in the present day, in fact, reflect the characteristics of the second modernity which they find significant. Despite the variations in their views, as Matthew Adams (2003, 2006, 2007) observes, they share a common assumption, which Adams labels as the extended reflexive thesis: "reflexivity is a burgeoning capability of modern subjects, which offers them the opportunity to construct self and self-relationships afresh" (Adams 2003, p. 223). The concept of reflexivity, in other words, plays a central role in their views of identity. But, as Giddens has noted, reflexivity, defined as "routinely 'keep in touch' with the grounds of what they do as an integral element of doing it" (Giddens 1990, p. 36) has always been a feature of humankind in all epochs of history; what distinguishes the reflexivity in late modern societies is "the fact that social practices are constantly examined and re-formed in light of incoming information about those very practices, thus constitutively altering their character... What is characteristic of modernity is not an embracing of the new for its own sake, but the presumption of wholesale reflexivity-which of course includes reflection upon the nature of reflection itself' (Giddens 1990, pp. 38-39).

\subsubsection{Giddens on the Self-Identity in Late Modern Societies}

The centrality of reflexivity, perhaps, is most salient in Giddens's view, who explicitly states that the self-identity in late modern societies is a "reflexive project [...] which consists in the sustaining of coherent, yet continuously revised, biographical narratives, take place in the context of multiple choices as filtered through abstract systems" (Giddens 1991, p. 5). In Giddens's view, the radicalised reflexivity in late modern societies extends well beyond the boundary of institutions and enters into people's lives through processes such as globalisation, which situate them in various high-consequence risks that are beyond their control. Constant exposure to high-consequence risks, in turn, generates a tremendous amount of existential anxiety. Although abstract systems allow people to be relieved from existential anxiety by sequestrating undesirable experiences and providing responses to possible risks, sequestration of experiences can never be complete. More importantly, abstract systems are themselves radically reflexive too, i.e. the responses they provide are themselves constantly contested. Hence, insofar as 
people's decisions and actions are mediated by abstract systems, they are inherently reflexive as well. The same goes for identity construction in late modern societies. Unlike traditional societies, in which people derive their identity from geographically local sites and/or relatively rigid social structures; the fixities once provided by places and traditions are lost in late modern societies. As such, people are required to actively construct a (more or less) coherent biography to sustain their self-identity. Since their choices are mediated by abstract systems, reflexivity becomes an inherent part of identity construction. Central to Giddens's reflexive project of the self, then, is people's coherent, yet constantly revising, self-narrative and their capabilities for active choice-making.

Giddens's substantive account of the nature of self-identity in late modernity is implicit in his analysis of Rainwater's 'self-help' manual, in which he deduces ten elements reflecting the nature of self-identity in late modern societies. They are:

(i) The self as a reflexive project, which the individual is responsible. We are [...] what we make of ourselves... [S]elf-understanding is subordinated to the more inclusive and fundamental aim of building/rebuilding a coherent and rewarding sense of identity...

(ii) The self forms a trajectory of development from the past to the anticipated future... The trajectory of the self has a coherence that derives from a cognitive awareness of the various phases of the lifespan...

(iii) The reflexivity of the self is continuous, as well as all-pervasive. At each moment [...] the individual is asked to conduct a self-interrogation in terms of what is happening...

(iv) [Self-identity,] as a coherent phenomenon, presumes a narrative: the narrative of the self is made explicit... [The narrative] is something that has to be worked at, and calls for creative input as a matter of course.

(v) Self-actualisation implies the control of time [by] establishing of zones of personal time [and, so far as possible, the] future is to be ordered by exactly those active processes of temporal control and active interaction on which the integration of the self's narrative depends.

(vi) The reflexivity of the self extends to the body, where the body is part of an action system rather than merely a passive object... [B]ody awareness is presented [...] as a means of constructing a differentiated self,... 
(vii) Self-actualisation is understood in terms of a balance between opportunity and risk... [where, the secular consciousness of risks is] inherent in calculative strategies to be adopted in relation to the future.

(viii) The moral thread of self-actualisation is one of authenticity, [...] based on 'being true to oneself [...] it means also disentangling [...] the true from the false self... To be true to oneself means finding oneself, but since this is an active process of self-construction it has to be informed by overall goals - those of becoming free from dependencies and achieving fulfilment...

(ix) The life course is seen as a series of 'passages'... [The passages] are not institutionalised, or accompanied by formalised rites... [The] transitions are drawn into, and surmounted by means of, the reflexively mobilised trajectory of self-actualisation.

(x) The development of the self is internally referential: the only significant connecting thread is life trajectory as such... The key reference points are set 'from the inside'... (Giddens I99I, PP. 74-80)

Although Giddens has not explicitly formulated a substantive account of the nature of self-identity in late modernity, the deduced elements allow us to reconstruct a substantive account of the self implicit in his analysis. In Giddens's view, the self is viewed as an active subject, both in the sense of action and in the sense of deliberation. Indeed, Giddens is describing a subject as someone who can, and must, actively "make" himself (in (i)) by conducting regular self-interrogation (in (iii)), working on his self-narrative (in (iv)), ordering the future (in (v)), and constructing a differentiated self via increased body awareness (in (vi)). Implicit in Giddens's description, however, is that subjects are disengaged from their life, i.e. the subjects and their self-identity (and self-narrative) are essentially distinct, in which the latter has to be "made", "formed", "constructed" or "mobilised" by the subjects. Giddens's reflexive self is equally active in deliberation, subjects are described by Giddens as if they are the engineers of their lives who ought to determine how to live their lives by their own designs. In Giddens's own terms, the construction of self-identity is about lifestyle choices and strategic life-planning, in which a subject has to reflectively choose from a plurality of lifestyles and to reflexively devise plans of the future in terms of his personal biography. The guiding principle behind the choices made by the reflexive self is, interestingly, instrumental rationality; it is exemplified by Giddens's emphasis on "cognitive awareness" (in (ii)), "active temporal control and active interaction..." (in (v)), "the calculate strategies" (in (vii)), and "self-construction has to be [...] informed by overall goals" (in (ix)). 
Moreover, execution of the reflexive project involves various cognitive capabilities, in particular the ability to balance trust and risks. Despite Giddens's insistence of the pervasiveness of abstract systems in people's lifestyle choices and life-planning, his emphasis on the subjects' radicalised reflexivity, their capabilities to shape their lives and subject-centeredness offers a conspicuous display of Taylor's notion of the disengaged self.

The similarity between Giddens's reflexive self and Taylor's disengaged self is particularly striking if we consider Giddens's view on the subject in (i) and the body in the reflexive project of the self in (v). The view, which sees persons, including both their self and body, to be something that are up for them to remake is precisely what Taylor refers to the Lockean punctual self. As reflexivity extends from the self to the body, and precisely because of such an extension, it makes the body as an object for remaking too. In light of this, the entire person appears to be an entity that can be, and indeed is, built from scratch. Hence, Giddens's reflexive self exhibits the traits of the disengaged self, i.e. the reflexive project of the self is one of self-mastery and self-making, which involves disengagement from oneself. It also places utmost importance on (instrumental) rationality in carrying out the project.

In my discussion of Giddens's analysis, I have tried to show that radicalised reflexivity appears to push the reflexive self towards the Lockean punctual self. It is, in some senses, an expected consequence: as radicalised reflexivity challenges the very stability of the self; in order to face these challenges, the subject has to constantly restructure the self, and together with the radicalised disembedding mechanisms in late modern societies, which have detached certainty from local contexts and traditions, the instability of the self is driven to the extreme. As Taylor has already noted, the Lockean punctual self is precisely an extremised disengaged self. Finally, it is also worth noting that Giddens has stated that the reflexive project of the self is also about authenticity. However, the notion of authenticity in Giddens's analysis (in (viii)) should be distinguished from the ideal of authenticity in the expressive self, because the notion of authenticity in Giddens's analysis is rationally driven. Although Giddens has stressed individuality and originality of the subject in his analysis, they are to be attained by a subject's reflective and reflexive shaping of his life. In other words, the notion of authenticity is defined by the subject's cognitive capabilities for overcoming the challenges of late modern societies. 


\subsubsection{Beck et al. on Identity in Late Modern Societies}

Like Giddens, Beck and his collaborators argue that changes in the institutions of modernity as well as its organising principles in late modern societies have significantly altered the mechanisms of identity construction (see, e.g. Beck \& Beck-Gernsheim 2002, Beck et al. 2003). Beck et al. claim that in late modern societies, people have become "a choice among possibilities, [i.e.] homo optionis" (Beck \& Beck-Gernsheim 2002, p. 5). By this, they mean that people are compelled to make choices for themselves in every aspect of their lives. Accordingly, people's lives in late modern societies are of the type of "living one's own life" (Beck \& BeckGernsheim 2002, Chapter 2). Beck et al., however, warn us not to mistake homo optionis and "living one's own life" to be something akin to the Lockean punctual self. On the contrary, subjects in late modern societies are what Beck et al. calls "institutionalised individuals": even though the subjects are compelled to choose and act on their lives, their choices and actions are constrained by the (new) guidelines and rules of the institutions of (late) modernity, e.g. the state, the job market, etc. (Beck \& Beck-Gernsheim 2002, p. 11). Moreover, although, in some sense, the subjects in both the first modernity and the second modernity are constrained by institutional structures, what distinguish institutionalised individuals in the second modernity is that: unlike the first modernity, where programmatic individualisation remains in force and the subject's identity is still mainly supplied by social institutions, social institutions in the second modernity no longer provide such sources to the subject's identity construction. When subjects in the second modernity are required to make choices, they can only rely on themselves. It is what Beck and Beck-Gernsheim call "a shift from normal biography to 'elective biographies', 'do-it-yourself biographies', risk biographies, broken or broken-down biographies” (Beck \& Beck-Gernsheim 2002, p. 24). On the other hand, the institutions of second modernity constantly demand the subjects for their own decisions and actions among numerous possibilities for issues that matter to them significantly. In other words, identity construction itself has become part of the social structure in second modernity (Beck \& Beck-Gernsheim 2002, p. xxii).

The new mechanisms of identity construction, as Beck et al. observe, are the product of the very structures of late modern societies, in which the society is fragmented into different and conflicting spheres, e.g. family, gender, job, etc. which only partially enclose the subjects. Travelling through these often opposing and contradictory spheres, people's identity is constantly unstable. Since society no longer helps the subjects to maintain a coherent identity, subjects have to take charge of their own lives and to live on their own. Beck and Beck-Gernsheim 
attribute the cause for the formation of this new form of identity to three specific processes: (i) globalisation, (ii) detraditionalisation, and (iii) individualisation.

Globalisation displaces subjects from specific geographical locations, and places them into multiple global locations. With globalisation, the subjects are not only affected by what is happening in one place, they are now connected to multiple places, where new questions, risks, and challenges in remote locations affect them equally. When the default status of the local is gone, a multiplicity of locations means that tensions and conflicts in different places have all become relevant to the subjects. At the same time, it also means that the subjects have to constantly choose from and act on issues that arise from the tensions and conflicts formerly remote and irrelevant to them. Similarly, detraditionalisation, i.e. traditions become something which is meaningful to the subjects only insofar as they are chosen and/or invented by the subjects themselves, strips away the certainty traditions once provide to the subjects. Finally, because of individualisation, i.e. "the disembedding without re-embedding" (Beck \& Beck-Gernsheim 2002, p. xxii), the subjects must actively search for their own identity. Active construction of identity, in turn, means that subjects are deemed to be self-responsible for their lives. Given the multiplicity of choices and actions, the disintegration of fixities, and the need to actively live one's life, individuality is central in this new form of identity because identity cannot be derived from any pre-given sources, overcoming these issues essentially means that the subjects have to create their own identity.

Beck et al.'s view of identity in late modern societies appears to be a mix of the disengaged self and the expressive self. In Beck et al.'s account of the second modernity, the world is filled with questions, risks, and challenges, and living in such a world successfully requires the subjects to be "active, inventive and resourceful, to develop ideas of one's own, to be faster, nimbler and more creative" (Beck \& Beck-Gernsheim 2002, p. 26). These qualities and capacities, in turn, presuppose a self-determining subject, who can manage and organise his life with (instrumental) rationality. At the same time, individuality also assumes an important role in Beck et al.'s view of the late modern identity. When places and traditions cannot provide meaning to the subjects, they have to create their (personal) meaning on their own. Accordingly, as Beck et al.'s view of "living one's own life" implies, every life differs. Still, it is important to note that the notion of individuality also takes on a different meaning as it is in the expressive self. Here, individuality does not only amount to self-expression or self-articulation; to be sure, self-expression and self-articulation are too involved in "living up to one's individuality". However, being an individual is primarily viewed as a cognitive task. 
Hence, behind individuality, it is instrumental rationality but not self-expression and self-articulation which matters more.

Beck et al.'s (2003) seemingly places the subjects at the core of the late modern identity. However, interestingly, and somewhat paradoxically, it is not the case in their view. Globalisation, detraditionalisation, and individualisation have dissolved the definite boundary of the subjects. Partial engagements in diverse but conflicting spheres result in an unstable and changing subject which can only be partially in charge of his own self-narrative. Because of that, Beck et al. argue that the subject in second modernity is not a unitary whole, it is now replaced by the "quasisubject", which is the "result as well as the producer of its networks, situation, location and form" (Beck et al. 2003, p. 25). Although the (quasi-)subjects are still compelled to make decisions and act, their (rational) decisions and actions alone cannot guarantee any specific outcomes; or, as Beck et al. suggest, "the subject is transformed into a constitutive part of a context that determines its subjectivity, and within which it exercises joint decision-making power" (Beck et al. 2003, p. 26; my emphasis).

Beck et al. argue that the rise of the quasi-subject results in a demise of individual responsibility and leads to (new) ethical questions. Their argument is correct, I think, insofar as individual subjects are taken as the sole unit for moral consideration. However, I want to highlight two important implications from the shift from subject to quasi-subject. Since quasi-subjects presuppose reciprocal relations with "the networks, situation, location and form", the networks, situation, location and form become normatively significant because they interfere with the life of people directly. More importantly, the notion of quasi-subject opens up a new conceptual space for assigning responsibility, as well as moral status, to nonsubjects, e.g. things. As quasi-subjects' agency can only be defined within and together with the larger context(s) of which the quasi-subjects have become a part of it. It means that responsibility of quasi-subjects can only be evaluated by including the non-subjects in the context(s). It, therefore, requires us to bring nonsubjects into moral consideration too.

\subsubsection{Taylor versus Giddens and Beck et al.: Affinities and Discontinuities}

To summarise this section, I want to review the affinities and discontinuities of the nature of the self in Taylor's and Giddens's and Beck et al.'s account. In Taylor's account, the modern self is comprised by the features of the two strands of the modern self, i.e. the disengaged self and the expressive self. Whereas the disengaged 
self is characterised by an appeal to (instrumental) rationality, the expressive self is characterised by an appeal to people's unique inner nature. The role of (instrumental) reason and, in a qualified manner, the uniqueness of individuals remains central in Giddens's and Beck et al.'s accounts. In their views, radicalised reflexivity in late modern societies requires the subjects to employ their cognitive resources to actively overcome the challenges in late modern societies. Since the structural and institutional changes in late modern societies have deprived individuals of the identity once provided to them by places and traditions, they have to live the life of their own - a life that is unique to them. In this sense, late modern identity is just a continuation of modern identity as described by Taylor.

However, the self has also undergone a thorough transformation in its very nature due to the structural and institutional changes in late modern societies. A new way to answer the question of 'what is my self-identity?' is needed. Here, I want to highlight four related features th2at are distinctive to the late modern self. Firstly, as Giddens and Beck et al. have constantly stressed, late modern societies are fragmented. As such, the stability and coherence of the subjects are challenged severely. In other words, the self as a stable and unitary entity cannot be taken for granted anymore. Fragments of the self spread into different spheres and can only be united by a self-narrative. While the subjects still have a role in constructing their self-narratives, they do not have the certainty once available. Secondly, the subjects are to be replaced by the quasi-subjects in which subjects constitute only a part of their agency. As a result, the scope of moral consideration has to be extended beyond the boundary of the subjects and to include the wider context(s) as well. Thirdly, the nature of the ideal of the late modern self has also been altered. The ideals of the disengaged self and of the expressive self are thought to be epistemologically grounded. This means that the ideals can be justified either by reason or the subject's unique nature. In other words, the ideals can be attained, in principle, either by reason(ing) or by self-expression or self-articulation of the inner nature. In this respect, the ideals are epistemologically self-sufficient. Radicalised reflexivity, however, puts such an epistemological self-sufficiency into doubt. Radicalised reflexivity challenges the legitimacy of the epistemological ground(s) of any ideals. It should be noted that, of course, challenges to epistemological grounds have always existed; but, the challenges in late modern societies are special in that they are internal. The way to attain the ideals in late modern societies, therefore, are seen as epistemologically open, as different epistemological grounds are considered to be equally (in)valid. Finally, related to the third feature, the ideal of 
the late modern self are constantly in flux, as opposed to the ideals of the disengaged self and of the expressive self, which are fundamentally fixed. ${ }^{8}$

\subsection{Living a Good Life in Modern and Late Modern Societies}

In surveying Taylor's account of modern identity, I have summarised two strands of the modern self. The ideals of the two strands of the modern self, as I have shown, are derived from the features of the modern self-interpretation and selfunderstanding. These ideals, in turn, are the basis of normative judgements. The ideal of the disengaged self gets support from the successes of scientific practices, and the ideal of the expressive self gets normative and evaluative force from various forms of artistic imagination. Either way, the subject-centeredness, the stability of the subjects and the notion of certainty are at work in the two strands of the modern self. Self-interpretation and self-understanding via these features, however, are discredited in late modern societies. Emerging from a different interpretation and understanding of the self in late modern societies is a different set of values. In this section, I shall connect the notion(s) of the self I have discussed with the values of modernity and late modernity. I explicate the values we can find in the notions of the self I have discussed, and examine the empirical research in light of my explication.

\subsubsection{The Modern Self, the Late Modern Self and the Criteria for the Good Life}

We can see two sets of values emerging from Taylor's historical narrative and interpretative explanation. To start with, knowledge-especially practical knowledge and technical knowledge-is presumed by the disengaged self to be universally valuable for all human beings. It is believed that only through acquisition of (practical and technical) knowledge people can exert control over their surroundings. An understanding of knowledge as such, in turn, presumes specific standards that are modelled on scientific practices. So construed, anything that fails to contribute to the development of practical or technical knowledge or to

\footnotetext{
8 While it is relatively uncontroversial to maintain that the disengaged self's ideal is fixed, it may be less obvious to the expressive self. For the expressive self, its ideal appears to be different from one individual to another and, therefore, it may give an impression of being in flux. However, individual differences and being in flux need to be distinguished carefully. In the expressive self, the individual differences lie in an individual's inner nature, which is still considered to be given and fixed.
} 
satisfy the standards of scientific practices, e.g. clarity and distinctness, are viewed as inferior. It is so, since human progress is to be assessed by the degree of control people have over their surroundings.

The flip side of control, of course, is an emancipation of humankind from being controlled. Autonomy, therefore, is another value that is fundamental to the disengaged self. Here, an autonomous being is defined as a subject, whose assent of his own actions, as well as the rules for those actions, must be the result of his own rational reflection. In other words, to be an autonomous being, one must also be a rational being. Since it is a subject's rational nature that constitutes his autonomy, the subject is deemed to have determined his own course of action and, therefore, is also solely responsible for his choices and actions. From the outlook of the disengaged self, every subject must be a rational being. And, precisely because of this, every subject is in essence the same and therefore, ought to be treated equally and impartially. Moreover, (rational) decisions and actions of different subjects should be viewed acceptable because they are justified by the same rational standard(s). This gives rise to a sense of tolerance because so long as they are (rationally) justified, people's decisions and actions are legitimate whether they are liked by others or not. In sum, knowledge, autonomy, equality, impartiality and tolerance are the key values promoted by the disengaged self.

The importance of autonomy and equality is too shared by the expressive self. Underlying the ideal of the expressive self is the notion of authenticity, i.e. to be true to one self. To be authentic, subjects must be free from externalities and to be able to decide and act by their own will. While the disengaged self defines the subject with universal reason, the expressive self points to individuals' differences. In such an outlook, individuality is to be valued. Accordingly, every individual should be considered as equal because of their uniqueness. Authenticity, together with respect for individual differences, lead to a valuing of diversity: the subjects ought to be respected by and recognised as who they really are. Diversity, therefore, ought to be preserved.

For the expressive self, its ideal can only be attained through self-expression or self-articulation of an individual's unique inner nature. However, (self-)disclosure as such is not a form of reason(ing) as in the disengaged self, but it is a form of creative endeavour. Creativity, therefore, is another key value of the expressive self. Although creative endeavour is not to be guided by (instrumental) reason, it does not mean that such an endeavour is without any standards. As Taylor noted, for the purpose of self-discovery, creative endeavour has to be epiphanic, where "the locus of a manifestation which brings us into the presence of something which is 
otherwise inaccessible" (Taylor 1989, p. 419). In other words, to satisfy the standard(s) of the expressive self, people's creative effort cannot be merely representational, it must be revealing as well. In sum, the key values of the expressive self are authenticity, autonomy, individuality, equality, diversity and creativity.

Besides the ideals (and the basic values) embodied in the two strands of the modern self, the ideals in the modern self are also shaped by the affirmation of ordinary life. The affirmation of ordinary life is a re-valuation of ordinary, mundane activities for which they are no longer considered to be inferior. The revaluation as such does not only redefine the scope of activities that are considered as valuable, it also characterises a particular way of life that is regarded as good. To illustrate this point, let us briefly turn to Max Weber's (2001/1930) discussion of Protestant ethic. In Protestant ethic, the moral status of working has been transformed with the new notion of calling. As I have explained, the kinds of activities in themselves do not determine whether a person's life is valuable or not; rather the value of a person's life is now measured by how he does those activities. So construed, work (and wealth acquisition) has become the end in itself, as Weber stated, "[1]abour must, on the contrary, be performed as if it were an absolute end in itself, a calling" (Weber 2001, p. 25). If work (and wealth acquisition) is seen as morally good, then characters which enable a person to work better, e.g. such as honesty, punctuality, industry, and frugality, are also seen as virtuous (Weber 2001, p. 17). In other words, the affirmation of ordinary life, particularly as it is advanced by Protestant ethic, promotes a particular set of personal characters as virtues.

Many of these values are retained by the late modern self. As I have argued, in an important way, the late modern self is a continuation of the modern self. Knowledge, autonomy, equality, tolerance, authenticity, individuality, diversity, creativity, etc. remain as important values for the late modern self. However, the new mechanisms of identity construction via radicalised reflexivity in late modern societies have challenged the status of these values. Neither scientific rationality nor epiphany can provide unquestioned ways to the ideals. Given the conditions of late modern societies outlined by Giddens and Beck et al., certainty and standardised criteria become less, or even not, relevant for evaluations of a person's life. As radicalised reflexivity compelled the subjects to construct their self-narratives by lifestyle choices and life-planning, choices of and for individuals have become the most important value for the late modern self. Indeed, Giddens argues that there is a shift from "emancipatory politics" to "life politics", where the former seeks individual liberation from (pre-)existing constraints, and aims to "reduce or 
eliminate exploitation, inequality and oppression" by "the imperatives of justice, equality and participation" (Giddens 1991, p. 211 \& 212), the latter is "a politics of lifestyle" that concerns itself with the question of 'how shall we live?'. To translate Giddens's characterisation of the political agenda of these two forms of politics into terms of values, I think, it is possible to see emancipatory politics as representing Taylor's view of modern identity, especially the disengaged self. Values such as knowledge, autonomy and equality are being strived for to free people from any pre-given natural and social orders. These values are important because they are about people's life chances. Once people are liberated from these constraints, they are propelled to consider the questions concerning their self-actualisation. As such, life politics represent an increasing emphasis on values such as authenticity, individuality and diversity.

I have provided a brief sketch of the basic values embodied in the modern and late modern self. This is admittedly a rather simplistic picture. But, I think the values I have illustrated are sufficient for the present study. To reiterate the aim of this chapter, my aim is to illustrate the ideals (and the basic values) embodied in the modern and late modern self, which can then allow us to have a better understanding of people's normative judgements concerning the issues related to the good life.

\subsubsection{The Shift of Values in Modern and Late Modern Societies}

My aim in the previous section was to illustrate the different sets of values in modern and late modern societies. We can also find empirical research that lends support to the claim that the basic values of modern and late modern societies are different. Using the data from a series of World Value Survey (WVS), Ronald Inglehart and his collaborators (see, e.g. Inglehart 1990, 1997; Inglehart \& Norris 2003, 2004; Inglehart \& Welzel 2005) argue that there is a coherent shift of values from non-industrial societies to industrialised societies and from industrialised societies to post-industrialised societies. The shift of values, they argue, is primarily a result of economic developments in modern societies and late modern societies, i.e. the development to industrial and post-industrial societies respectively. To demonstrate the shift of values empirically, Inglehart et al. have amassed a large amount of data verifying that in the richer and more developed countries, people place a higher emphasis on values related to subjective well-being, even at the expense of further economic growth. And, they also demonstrate a shift away from political, economic and scientific authority to personal freedom (Inglehart 1997, pp. 
39-45). In this section, I discuss Inglehart et al.'s works, and I argue that their findings verify my characterisation of the views of the good life in modern societies and late modern societies. Particularly relevant to my purpose, is Inglehart's study of a cultural shift from materialist values to postmaterialist values, which is "part of a broader syndrome of [...] cultural change, in which a growing emphasis on quality of life and self-expression is accompanied by a declining emphasis on traditional, political, religious, moral, and social norms" (Inglehart 1990, p. 66).

In his study of the cultural shift, Inglehart distinguishes two spectra of values, i.e. traditional values vs. secular-rational values and survival values vs. selfexpression values. ${ }^{9}$ To explain the shift from traditional values to secular-rational values, as well as the shift from survival values to self-expression values, Inglehart connects these shifts to different economic developments. He argues that the shift to secular-rational values is primarily a result of industrialisation, while the shift to self-expression values is a result of the rise of post-industrialised societies. Important in Inglehart's theory is the connection between values, risks and security, and economic developments. These concepts are joined together by Inglehart's Scarcity Hypothesis: “an individual's priorities reflect the socioeconomic environment; one places the greatest subjective value on those things that are in relatively short supply" (Inglehart 1997, p. 33). ${ }^{10}$

According to Inglehart, in pre-industrialised societies, the mechanisms against risks are relatively underdeveloped. Hence, pre-industrialised societies are characterised by a high physical and economic insecurity. In these societies, social stability is necessarily for people's survival. Therefore, people value strict social hierarchy, absolute authority, communal obligations, etc. which promote social stability. Tradition values are important also because they offer an answer to the existential anxiety arising from the insecurity. As industrialisation (and modernisation in general) has proven to be a better way to combat physical and economic insecurity, the things that have enabled people in industrialised societies

9 In the earlier studies, e.g. Inglehart (1997), Inglehart uses the term 'subjective wellbeing' instead of 'self-expression values'.

10 In his explanation of the value shifts, Inglehart has also mentioned the Socialisation Hypothesis, i.e. "[t]he relationship between socioeconomic environment and value priority is not one of immediate adjustment: a substantial time lag is involved because, to a large extent, one's basic values reflect the conditions that prevailed during one's preadult years" (Inglehart 1997, p. 33). However, the temporal (and, intergenerational) dimension of the value shifts is not of direct relevance for this section. 
to cope with their economic and physical insecurity are also being valued. Underlying industrialisation is a set of beliefs that prizes rationality, personal achievement and economic prosperity. By deploying (instrumental) rationality that is prototypical of science and technology, people become more capable of controlling their surroundings and, thereby reducing physical insecurity. Moreover, industrialised societies are characterised by their stress on efficiency and productivity, which are derived from the work ethic I have previously explained. By adhering to the principles of work ethic, people may obtain personal achievement and economic prosperity. So, the personal characters highlighted in work ethic are credited because they allow people to reduce economic insecurity. In Inglehart's account, the reduced physical and economic insecurity in industrialised societies helps to explain the shift from traditional values to secularrational values.

However, neither scientific rationality nor economic affluence provides personal meaning to people. Once physical and economic insecurity are removed by further economic developments in late modern societies, there is a rising need for the quest of personal meaning. In other words, as people can satisfy their material desires more easily and the limits imposed by physical environment are lessened, their focus has shifted from mere economic affluence to the need for self-expression and subjective well-being. It is because self-expression and subjective well-being become relatively short in supply in comparison to pure materialistic satisfaction. The room for tolerance, diversity and ambiguity is also greater in post-industrialised societies because the need for an absolute has diminished in the relatively secure environment in those societies.

Inglehart's description of the shift of values from traditional values to rationalsecular values and from survival values to self-expression values fits nicely with the values of the modern and late modern self. The modern self, particularly the disengaged self, is one that emphasises rational-secular values. It is especially so in its emphasis on certainty and standardised criteria. And, with the affirmation of ordinary life and the influence of Protestant ethic, the modern self places high priority on materialistic satisfaction and economic affluence. While the late modern self emphasises personal growth, which is dissociated with pure materialistic satisfaction or economic affluence. To be sure, as Inglehart et al. and Giddens point out, materialistic satisfaction or economic affluence per se are not being disvalued in late modern societies. The shift towards self-expression values or the shift towards life politics requires an adequate development into post-industrialised or late modern societies. But, once this shift has occurred, things that go against self- 
expression values or against the political agenda of life politics will be seen as detrimental.

Inglehart et al.'s account has placed primary emphasis on economic development as the cause for the shift(s) of values. While I agree with Inglehart et al. that economic developments have provided the context for the reorientation(s) of values from traditional values to rational-secular values and from survival values to self-expression values, I think their emphasis on economic developments can be misleading. I think economic developments only contribute indirectly to the shift(s) of values. It is, indeed, true that the economic developments in modern and late modern societies help to reduce or eliminate physical and economic insecurity; and, thereby providing the necessary condition for people to reject traditional values in modern societies (and rational-secular values in late modern societies). However, economic developments by themselves do not provide the conceptual tools for articulating and defending the shift(s). In this respect, I think Inglehart et al.'s emphasis on economic developments can be misleading.

Still, it is worth noting that Inglehart et al. have also argued for the indispensable role of cultural heritage in the shift(s) of values. It is the cultural heritage, I think, that provides the conceptual tools for articulating and defending the different sets of values. As I have shown, through my discussion of the works of Taylor, Giddens and Beck et al., different sets of values are justified by different notions of the self. It is only by referring to the notions of the self, can we coherently articulate and defend the sets of values we find legitimate. Here, the dominant values in modernity are derived from the two strands of the modern self. And, the values of late modern societies are based on the notion of the reflexive self (and quasi-subject). In other words, although I agree with Inglehart et al.'s claim that there is a shift of values in modern societies and late modern societies, I disagree with his primary emphasis on economic developments. Nevertheless, I believe, Inglehart and his collaborators have provided useful empirical support to my discussion of the views of the good life in modern societies and late modern societies.

\subsection{Conclusion}

In this chapter, I have examined the modern and late modern self presented by Taylor, Giddens and Beck et al. I have shown that these notions of the self have embodied their own sets of basic values. Through Taylor's account of modern identity, I have summarised two stands of the modern self, i.e. the disengaged self and the expressive self. Together, they give us two sets of basic values consisting of 
knowledge, autonomy, equality, impartiality, tolerance, individuality, diversity and creativity. Then, I have turned to Giddens's and Beck et al.'s works on the new mechanisms of identity construction in late modern societies, and have illustrated the shift from a notion of the self that is stable, unitary and certain to one that is characterised by instability, fragmentation and uncertainty. The late modern self, I contend, does not fully reject the ideals in the earlier modernity, but it does put the ground for those ideals into question. In other words, while the basic values embodied in the late modern self remains very much the same as those in the modern self, the ground for those basic values has become more contingent. 



\section{CHAPTER THREE}

\section{From Culture 2.0 to a Network State of Mind} A Selective Analysis of Web 2.0's Axiologies

To summarise the study so far, I have argued for a Walzerian approach to digital media and the good life, which requires researchers to engage with actual discourses on digital media and the good life, i.e. prudential appraisals. I have also argued that the normativity of prudential appraisals is grounded in the ideals embodied in various notions of the self. Hence, to truly understand prudential appraisals, particularly to truly understand how prudential appraisals become credible and persuasive, it is necessary to identify their normative and evaluative resources. In the last chapter, I have identified the disengaged self, the expressive self and the reflexive self, as well as the ideals they embody through the works of Charles Taylor, Anthony Giddens and Ulrich Beck.

Now, it is time to move on and see how these notions of the self and the embodied ideals are at work in prudential appraisals. Here, I shall analyse a number of representative examples of prudential appraisals. This exercise is not only required by the Walzerian approach, it also helps to illustrate my claim that prudential appraisals are powered by specific ideals (or the views of the good life) embodied in different notions of the self I have previously identified. More importantly, this exercise can also deepen the understanding of our normative 
judgements on the impacts of digital media on our lives and provide hints on how normative judgements on digital media and digitally-mediated practices should be made, if they are to be effective at all. ${ }^{1}$

In this chapter, I restrict myself to the popular discourse on Web 2.0, because it (re)presents the latest concerns raised about the impacts of digital media on our lives. In the next section, I begin with a brief introduction of Web 2.0 in Section 3.1. An introduction to Web 2.0 is necessary, as the recent discourses on digital media and the good life are tied to the key characteristics and features of Web 2.0. In Section 3.2 and Section 3.3, I examine two sets of prudential appraisals using the notions of the self I have identified in the previous chapter. The first set of prudential appraisals discusses the impacts of digital media on culture and society, which influence people's lives by transforming the exteriors of their lives; and, the second set of prudential appraisals discusses the impacts of digital media on our brain, mind and/or the self, and how digital media influences people's lives by transforming their interior lives. More specifically, I show that prudential appraisals of digital media can be better understood through a philosophical (re)interpretation via different notion(s) of the self. This, I argue, has an important implication to normative analysis of digital media and the good life, namely we should (re)direct our attention to the question: who we should be in a digitally-mediated world?

Unfortunately, I do not think that there is a definitive answer to this question. And, I shall not pretend to have an answer fully worked out here. Still, I believe a closer look at this question will be fruitful. Particularly, we can look at the nature of this question, as well as how this question should or should not (or, can or cannot) be answered. I examine this question in Section 3.4, and discuss some considerations in our answering of this question.

\footnotetext{
1 Some have already examined actual discourses (i.e. popular discourse) on digital media via different perspectives and approaches. Most notable among them are Coyne (1999), Mosco (2004), Flichy (2007), Barbrook (2007). Recently, Fisher (2010) has offered an ideological critique of network capitalism (or techno-capitalism) through an analysis of what he labels as "digital discourse". Fisher's study is, perhaps, most similar to the present study. Yet, his study differs from the present study in that his account is explicitly Marxist and his focus is primarily on social, political and economic underpinnings of network capitalism, whereas the present study is not. In a nutshell, what distinguish the present study from the others are my explicit focus on the good life and my approach to normative analysis, in which various notions of the self play a central role.
} 


\subsection{A Short Introduction to Web 2.0}

There is never a shortage of celebratory and condemnatory popular discourse on the Internet-even in its early days. ${ }^{2}$ But, the hopes and dreams of the Internet have faded with the burst of the dot-com bubble in the 1990s and the rise of control and surveillance over and through ICTs after September 11, 2003 (Geert 2011/2003, pp. 5-7). These events have momentarily quieted the popular discourse, as they seemed to have confirmed the worry and doubt raised by alarmists of the Internet. Yet, the advent of Web 2.0, with its newly proclaimed potential and promise, has rejuvenated the hopes and dreams of enthusiasts of the Internet and renewed the popular discourse. Looking at some of the most representative examples available, Section 3.2 and Section 3.3 are devoted to this (new) wave of popular discourse, focusing on the (new) worries and doubts voiced by alarmists and the (new) hopes and dreams portrayed by enthusiasts.

I will examine the axiologisation (i.e. what benefits and harms are being foregrounded in prudential appraisals) and problem representation (i.e. what problems are being represented in prudential appraisals) of Web 2.0 in popular discourse. My analysis aims to disclose what values are being employed in support of and/or against Web 2.0, and, in turn, to unveil the normative and axiological foundation of various prudential appraisals. Of course, there is a wide range of methods for examining actual discourses. ${ }^{3}$ In the present study, I shall not formally align my analysis with any specific methods for analysing discourses. Instead, I will attempt to analyse the prudential appraisals from a philosophical perspective through a close (re)reading of them in light of the normative and evaluative resources I have presented in Chapter Two. In short, my analysis is guided by the idea of sense-making: why and how a prudential appraisal makes sense to us at all? In answering this question, I attempt to show why, and in what ways, different prudential appraisals can be taken as plausible. Here, I pay special attention to the values that lie beneath the prudential appraisals. In this way, I can also uncover what grounds the normativity of prudential appraisals.

Before proceeding to my analysis, however, it is useful to briefly outline what Web 2.0 is, especially how it differs from its predecessor, i.e. Web 1.0. Such a

2 For a brief and succinct overview of popular discourse on digital media and various normative positions on it, see Thierer (2011).

3 For a helpful overview of various approaches and methods of discourse analysis, see Glynos et al. (2009). 
review will help explain the renewed interest on the Internet in popular discourse; it will also help locate what is believed to be at stake by the enemies and friends of Web 2.0, which, in turn, will provide the background of my analysis.

As many researchers have acknowledged, to provide a definition of the term 'Web $2.0^{\prime}$ - by giving necessary and sufficient conditions for the usage of the term-is likely to be a hopeless pursuit (see, e.g. Song 2010, Fuchs et al. 2010, Fuchs 2010). Hence, I will only outline the common characteristics and features that can be found on various Web 2.0 applications that distinguish them from Web 1.0 applications. In other words, instead of providing a definition for the term, I will treat 'Web 2.0 ' as a family resemblance concept which ties together various Web 2.0 applications.

Even so, there is still a long list of diverse characteristics and features linked to Web 2.0 applications such as wikis, folksonomies, mashups and social networking sites, etc. Here, Tim O'Reilly's, i.e. the originator of the term 'Web 2.0' (Graham 2005), description of Web 2.0 will be helpful. He describes Web 2.0 as

[...] the network as platform, spanning all connected devices; Web 2.0 applications are those that make the most of the intrinsic advantages of that platform: delivering software as a continually-updated service that gets better the more people use it, consuming and remixing data from multiple sources, including individual users, while providing their own data and services in a form that allows remixing by others, creating network effects through an "architecture of participation," and going beyond the page metaphor of Web 1.0 to deliver rich user experiences. (O'Reilly 2005b)

O'Reilly's 'definition' of Web 2.0 highlights the immediate (i.e. "continuallyupdated"), dynamic (i.e. "consuming and remixing data from multiple sources"), open and collaborative (i.e. "providing their own [content] [...] that allows remixing by others") nature of Web 2.0 applications. The metaphor of Web 2.0 as a platform is especially illuminating here, as the term 'platform' implies that the Web is a place for exchanging content and an interface for (undesignated) activities. ${ }^{4}$

\footnotetext{
4 According to Oxford English Dictionary, the word "platform" refers to "[a] surface or area on which something may stand, esp. a raised level surface" (or, alternatively, "[a] raised level surface on which people or things can stand, usually a discrete structure intended for a particular activity or operation."
} 
Unlike Web 1.0, which is based on the metaphor of Web as a portal, i.e. the Web is (only) a gateway to information already made available (Ginnell 2009, pp. 577578). ${ }^{5}$ Accordingly, Web 2.0 distinguishes itself from Web 1.0 by its participatory nature. Indeed, a platform does not have to have content of its own, nor does it by itself direct you to certain pieces of information available; it becomes contentful only when users meet on the platform.

Of course, that is not to say that the users of Web 1.0 do not, or cannot, interact with each other. Interactions are possible on Web 1.0, e.g. through e-mails. However, Web 1.0, viewed as a portal, does not require users' contributions and interactions to survive and thrive. This is why Felicia Song (2010) suggests the locus of difference between Web 2.0 and Web 1.0 lies not in "what" the users do (or can do) on the Web or "what" Web 2.0 enables the users to do, but rather in "how" the users ought to approach the Web. A comparison between a platform and a portal will again be helpful here. Unlike a portal, which offers entrances to various destinations, a platform is a place for activities and meetings; it does not only invite people to participate, but its very existence is dependent on people's participation. As such, participation is not only something users do (or can do) on Web 2.0, but it is something they ought to do.

Hence, Web 2.0 as a platform also implies a shift in the role of users as well. Users are no longer seen as passive consumers of content; they are now seen as active creators of content. In short, the distinction between consumers and creators (or producers) is blurred in Web 2.0 (Beers \& Burrows 2007, Ginnell 2009; also see, Ritzer \& Jurgenson 2010). This difference in the role of users is another key difference between Web 2.0 and Web 1.0. But, again, this is not about "what" the users do but about "how" they do. In other words, it is not to say that users of Web 1.0 do not, or cannot, create content; but, content creation is dramatically different in Web 1.0 and Web 2.0. Particularly, users of Web 1.0 must master some coding skills if they are to create any content at all, whereas Web 2.0 has removed this technical requirement from the users. Also, the content created on Web 1.0 is static and read-only, whereas the content created on Web 2.0 is dynamic and (re)writable. The differences between Web 1.0 and Web 2.0 are best illustrated by Beer and Barrow (2007) with Figure 1.

5 Oxford English Dictionary defines "portal" as "[a] door, gate, doorway, or gateway, of stately or elaborate construction; the entrance to a large or magnificent building, esp. when emphasised in architectural treatment; any door or gate (chiefly poet.)." 
Figure I.

\begin{tabular}{|c|c|c|}
\hline $\begin{array}{l}\text { Dimension of } \\
\text { Differences }\end{array}$ & $\begin{array}{l}\text { Web I.0 } \\
(1993-2003)\end{array}$ & $\begin{array}{l}\text { Web 2.0 } \\
\text { (2004-beyond) }\end{array}$ \\
\hline Mode... & Read & Write and Contribute \\
\hline $\begin{array}{l}\text { Primary Unit of } \\
\text { Content... }\end{array}$ & Page & Post/record \\
\hline State... & Static & Dynamic \\
\hline Viewed through... & Web Browser & Anything \\
\hline $\begin{array}{l}\text { Content created } \\
\text { by... }\end{array}$ & Web Coder & Everyone \\
\hline Domain of... & $\begin{array}{l}\text { Web Designers and } \\
\text { Geeks }\end{array}$ & $\begin{array}{l}\text { A new culture of } \\
\text { public research? }\end{array}$ \\
\hline
\end{tabular}

Note. Adapted from "Sociology and Sociology and, of and in Web 2.0:

Some Initial Considerations", by David Beer and Roger Burrows (2007), Sociological Research Online, 12 (5), 30 September 2007.

Available Online at: http://www.socresonline.org.uk//2/5//7.html

To summarise this brief introduction to Web 2.0: Web 2.0 is distinct from Web 1.0 by its participatory nature, with immediacy, dynamic content, openness and collaborativeness as its key characteristics and features. Users of Web 2.0 also differ from users of Web 1.0 in that, with Web 2.0 applications, they are now viewed primarily as content creators rather than content consumers. Yet, it is important to be reminded that the participatory nature of Web 2.0 and the role of users as content creators are not merely descriptive, they are in effect Web 2.0's normative ideals.

\subsection{Enemies and Friends of Culture 2.0}

Having identified the characteristics and features of Web 2.0, I shall now proceed to my analysis of popular discourse on Web 2.0's impacts on the good life. There is a vast amount of popular works, either in print or online, on the (potential) impacts of Web 2.0. My intention is not to recount all accounts of Web 2.0 presented in this large and still burgeoning amount of works, which will hardly be manageable. Instead, my objective is to offer a philosophical (re)interpretation of popular discourse on Web 2.0 via a number of representative examples, and disclose the view(s) of the good life and the notion(s) of the self underline them.

My point of entry into the analysis is Andrew Keen's (in)famous The Cult of Amateur: How Today's Internet is Killing Our Culture (2007). Keen's book is especially suitable as the point of entry to the popular discourse not only because it 
is arguably the first book-length critique of Web 2.0, it is also because the book has generated an enormous amount of positive and negative responses from both the proponents and detractors of Web 2.0 as well as the public. As I have argued in Chapter One, critics and their criticisms become a proxy of the public when their judgements resonate with the public. ${ }^{6}$ The reception of Keen's book, therefore, should qualify it as a window to one of the major standpoints on Web 2.0.

\subsubsection{Web 2.0, the Enlightenment's Nightmare?}

The Cult of Amateur is a wholesale assault on Web 2.0 and the purported Web 2.0 culture and ethos, which Keen believes (will) have "destructive impact" on "culture, economy and [moral] values" (Keen 2007, p. 1). The culprits of cultural, economic and moral demises in Keen's criticism of Web 2.0 are the "democratisation of media" and "noble amateurs", as he believes that they will-if they have not already — come to displace the "traditional cultural institutions" and "experts and cultural gatekeepers". According to Keen, by providing users the means of production and distribution of text, music and video that are originally in the hands of a limited number of cultural institutions, Web 2.0 has enabled the "noble amateur", i.e. "a hobbyist, [...] someone who does not make a living from his field of interest, a layperson, lacking credentials, a dabbler" (Keen 2007, p. 36), to create and distribute free, self-made content. And, whereas the democratisation of media has taken away the traditional cultural institutions' vantage positions to control, regulate and filter information, the proliferation of amateur content and celebration of amateurism have crowded out expert's judgements and the importance of expertise (see, Keen 2007, Chapter 1 \& 2). ${ }^{7}$ In short, Keen's criticism of Web 2.0 is precisely a criticism of Web 2.0's participatory nature and the new role of users as active content creators.

The question remains is that even if it is true that there is a disempowerment of traditional cultural institutions and a decline in the importance of experts and expertise, what is wrong (and/or bad) with them? To answer this question, we have

\footnotetext{
6 See, Chapter 1, section 1.5 (esp., section 1.5.2)

7 Keen has also criticised other aspects of the Internet, e.g. piracy, intellectual property and plagiarism (Keen 2007, Chapter 4, 5 \& Chapter 6, esp. pp. 141-145), privacy and (participatory) surveillance (Keen 2007, Chapter 7), pornography (and sex) and gambling online and Internet addiction (Keen 2007, Chapter 6, esp. pp. 146-165). Pace Keen, I do not think these issues are unique to Web 2.0. Hence, I will not examine his discussion of those issues here.
} 
to understand how Keen conceives traditional cultural institutions and experts. For him, traditional cultural institutions are specific systems of content-or, more accurately, knowledge-production and distribution, which are characterised by rigorous review processes and standards; and, in turn, the rigorous review processes are carried out by experts (with their expertise). Moreover, expertise can only be acquired through extensive education and training made possible by traditional cultural institutions. So construed, in Keen's view, traditional cultural institutions and experts (and their expertise) are mutually dependent on each other (Keen 2007, Chapter 2).

Following this line of thought, Keen argues that the vice of Web 2.0 is a degradation of truth and trust. He claims that it is difficult to find truth on Web 2.0 because "in a flattened, editor-free world where [amateurs] can post their amateurish creations at will, and no [expert] is being paid to check their credentials or evaluate their material, media is vulnerable to untrustworthy content of every strip.” (Keen 2007, p. 19) In other words, his claim is that Web 2.0 lacks review processes and standards enforced by traditional cultural institutions and the experts they house, which are required for the acquisition of truth (or acquisition of knowledge) (Keen 2007, Chapter 2 \& 3). In the same vein, Keen laments the aggregation of users' (or, more precisely, amateurs') preferences and opinions through computational algorithms have come to replace traditional cultural institutions' review processes and standards as well as expert's judgements, as he believes the former is easily manipulable and unbalanced (Keen 2007, pp. 92-96).

Keen also claims that the democratisation of media and noble amateurs have resulted in a problem of trust, because Web 2.0 allows users to produce and distribute content anonymously, it is difficult-if not impossible-to verify its sources. Or, conversely, the content produced and distributed anonymously hardly requires supports because of its lack of verifiability (Keen 2007, pp. 65-75). In other words, anonymity allows users to run free from moral responsibility and legal liability (Keen 2007, pp. 71-83). A similar argument is offered by Keen in arguing against the practice of "remixes" and "mash-ups" on Web 2.0, too. In a nutshell, he argues that remixing and mashing-up content have destroyed the idea of authorship and integrity of works by encouraging fragmentation of authorship and works (Keen 2007, pp. 23-26, 57-60).

Keen's book has been thoroughly scrutinised by a number of commentators. For instance, Lawrence Lessig (2007) has argued that his book has suffered from numerous factual, interpretative and conceptual errors. Although the commentators 
of Keen's book may well be right in their criticisms, I am not interested in assessing Keen's criticism as such. Instead, my aim is to make sense of the normativity of his criticism of Web 2.0, which, as I have pointed out, can serve as a proxy for a wider standpoint on Web 2.0. Hence, I shall set aside the questions concerning its empirical and conceptual plausibility, and focus on the values he employs. ${ }^{8}$

It is not too difficult to see what values are at work in Keen's criticism of Web 2.0. His defence of traditional cultural institutions' review processes and standards and experts and expertise acquired through extensive education and training aligns his criticism with the Enlightenment's belief in the supremacy of scientific rationality. However, I think his criticism of Web 2.0 can still be better understood by (re)contextualising it via the image of the disengaged self I have previously elaborated. ${ }^{9}$ To begin with, his defence of traditional cultural institutions as well as experts and expertise against the democratisation of media and amateurism is a clear instance of championing (scientific) empiricism: neither user-generated content nor aggregation of users' preferences and opinions qualify as truth (or knowledge), because they fall short of the standards set by scientific practices as conventionally understood, i.e. clarity and distinctness. Alternatively, we can also view Keen's defence of experts and expertise as a defence of individual rationality in opposition to irrationality of the collective. In his account, experts are intellectual heroes who have access to truth and standards; and, they are heroic, because expertise is acquired through an individual's hard work in education and training (i.e. selfdisciplining). Expertise, in other words, is an individual's intellectual achievement as well as a manifestation of individual rationality par excellence.

On the other hand, Keen's criticism of anonymity and the failure of trust in Web 2.0 can also be better understood through the image of the disengaged self. Anonymity is wrong and bad because it offers an escape from moral responsibility and legal liability. With anonymity and fake identities on Web 2.0, people can act without assenting to their decisions and actions. However, the possibility of a person acting without assenting to his own decisions and actions goes against the core of the disengaged self, namely the idea of the self being an autonomous subject, i.e. a subject who is (self-)responsible and (self-)disciplined because of his own will. ${ }^{10}$ The same is true to his criticism of "remix" and "mash-ups" too, as his idea of

8 See, Chapter 1, section 1.4.2 for a discussion on the different dimensions of prudential appraisals.

9 See, Chapter 2, section 2.2.1 and section 2.4.1.

10 See, Chapter 2, section 2.2.1. 
author is a person who is, and can be, held responsible and accountable for the works he has produced. In short, for Keen and the standpoint his criticism represents, Web 2.0 is problematic because it fosters a class of sub-rational people (i.e. with the disappearance of traditional cultural institutions and experts) and nonautonomous subjects (i.e. with the withholding of moral responsibility through anonymity and fake identities) that run against to the ideal of the disengaged self.

\subsubsection{Web 2.0 and the (Post-)Enlightenment Dream}

In a sharp contrast to the condemnatory attitude towards Web 2.0 (re)presented by Keen, there is a camp of proponents who champion Web 2.0's revolutionary potential and promise, especially its participatory nature and the new role of users as active content creators. They have published a number of "manifestoes" to demonstrate Web 2.0's potential and promise, most notable among them are Yochai Benkler's Wealth of Networks: How Social Production Transform Market and Freedom (2006), Don Tapscott and Anthony D. Williams's Wikinomics: How Mass Collaboration Changes Everything (2006) and Macrowikinomics: Rebooting Business and the World (2010), Charles Leadbeater's We-Think (2008), and Clay Shirky's Here Comes Everybody: How Change Happens When People Come Together $^{11}$ (2009) and Cognitive Surplus: Creativity and Generosity in a Connected Age (2010). ${ }^{12}$ In this section, I will focus on the two books by Shirky. My choice of Shirky's books over the other titles is based on the subject of Shirky's books, which is about the impacts of Web 2.0 on society in general, and Shirky's visibility and popularity among the general public-as one reviewer of Cognitive Surplus writes, "Clay Shirky is the Internet's most prominent ponderer [...] Every six weeks or so the New York Times quotes Shirky in stories [...]" (Last 2010). Shirky, I believe, is a suitable representative for the celebratory attitude towards Web 2.0.

\footnotetext{
11 This title is first published in 2008 with the title Here Comes Everybody: The Power of Organizing without Organizations, and an updated edition with a new chapter is later published in 2009. The page numbers in this chapter refer to the pages numbers of the updated edition.

12 Some have criticised these manifestoes for equivocating non-profit and profitoriented (user-)production, thereby legitimatising an expansion of capitalism into the social, cultural and political dimensions of our life, see, e.g. van Dijck \& Nieborg (2009). While I agree that this line of criticisms is important to normative analysis of Web 2.0, my main goal of this chapter is only to examine the normative grounds of prudential appraisals
} 
Here Comes Everybody and Cognitive Surplus are parts of Shirky's larger project to illustrate the revolutionary potential and promise of Web 2.0. The two books, as Shirky himself notes, are written with two different agendas in mind: whereas the first book is "about the rise of social media as a historical fact, and the changed circumstances for group action [...]", the second book discusses "free time [as] a [new] shared global resource" made possible by social media and how to "take advantage of that resource" (Shirky 2010, p. 27). In this respect, Here Comes Everybody is mainly descriptive, i.e. it aims to describe what is of value in social media, while Cognitive Surplus can be viewed as normative in that it recommends specific ways to design and use social media to realise certain end.

The running argument of Here Comes Everybody is a simple one: social media (or Web 2.0), as a set of new communication tools, has lowered the socioeconomic cost of sharing, cooperation and collective action, and it has led to new ways of organising and running our society, such as "post-managerial organisation[s]" (Shirky 2009, p.41), a disappearance of "professional categories" (Shirky 2009, p. 70), a new system of (cultural) production based on "publish-then-filter" (Shirky 2009, p. 81), an "unmanaged division of labour" (Shirky 2009, p. 117) and a replacement of "planning with coordination" (Shirky 2009, p. 178), etc. And, the new ways of organising and running society, as Shirky argues, are beneficial to both the individuals and society. For the individuals, social media has increased freedom in both the negative and positive sense, i.e. individuals are free from management, (professional) authority and plan, and they are also free to participate, express (through publishing and producing content) and experiment. For the society, social media has helped to resolve social dilemmas through connecting individuals and make available to them previously untapped resources the previously unconnected people can provide.

In Cognitive Surplus, Shirky rehearses most of the points he has made in the earlier book, especially his claim that social media has allowed an increase in both negative and positive freedom, but his emphasis has shifted to positive freedom, i.e. what individuals can do-or, what individuals can do with the free time-in the context of the new degree of connectedness afforded by social media. The crux of Shirky's argument is that using free time for passive consumption is excusable only insofar as the socioeconomic cost of its alternatives is too high for individuals, but with the cost of active participation lowered tremendously by social media, passive consumption should no longer be the norm for using free time (Shirky 2010, pp. 1012). To highlight the potentials of active participation, Shirky asks his readers to "imagine treating the free time [...] as an aggregate, a kind of cognitive surplus" 
(Shirky 2010, p. 9) that "can be harnessed for large, communally created project, rather than as a set of individual minutes to be whiled away one person at a time" (Shirky 2010, p. 10). He argues that active participation is, in effect, a manifestation and realisation of people's sense of autonomy and competence at the personal level and their sense of membership and generosity at the social level (Shirky 2010, Chapter 3), and it is also a source of personal, communal, public and civic values, which passive consumption cannot provide (Shirky 2010, Chapter 6). In short, Shirky's argument is one against passivity and in favour of (inter)activity. In Shirky's view, being (inter)active has become an imperative; or, to put it differently, an imperative to participate (through producing) and sharing has come into being along with the rise of social media.

Shirky's praise of social media's capacity to increase freedom seemingly aligns his judgement with the ideal of the disengaged self, i.e. a notion of the self that prioritises autonomy. His celebratory note on the new positive freedom, i.e. the capability to participate (through publishing and producing) and share in every individual, also suggests an invocation of the value of equality: every individual is equal with respect to their opportunity to participate and share, which, again, seems to show that the normative and axiological foundation of his judgement is the ideal of the disengaged self. However, I contend that the disengaged self cannot make sense of (i) Shirky's positive attitude towards the unorganised (or disorganised), the rejection of professional authority (as in Keen's understanding) ${ }^{13}$ and improvisation, and, more importantly, (ii) the imperative of (inter)activity or the imperative to participate and share.

It should be relatively straightforward why the disengaged self cannot ground (i). Although the disengaged self prioritises autonomy, autonomy is valuable insofar as it is restrained by self-discipline. However, the unorganised (or disorganisation), the rejection of professional authority and improvisation imply a devaluing of self-discipline. Of course, certain degree of (self-)disciplining must be involved in performing any activities, be they managed or unorganised, governed/guided or ungoverned/unguided by authority, or planned or improvised. Yet, (self-)discipline in the latter are not (self-)discipline in the strong, rational sense required by the disengaged self; they are (self-)discipline only in the sense that those activities are willingly chosen and acted upon, i.e. they are only performed by a person's own liking and can be abandoned easily. Moreover, the plea for the unorganised, the

13 See, section 3.2.1. 
rejection of professional authority and improvisation also presumes a fluid worldview that is incompatible to the disengaged self, which presupposes a stable worldview. ${ }^{14}$

Also, the disengaged self cannot make sense of (ii) because (inter)activity or participation and sharing cannot be viewed as an imperative from its perspective. It is indeed true that participation and sharing are valued from the perspective of the disengaged self, e.g. Kant, one of the key Enlightenment thinkers inclined towards the disengaged self, argues that the exchange of well-reasoned thoughts through participation and sharing in the public realm is necessary for universal enlightenment (Frick \& Oberprantacher 2011, pp. 18-19). However, the argument for participation and sharing can only be negative, i.e. it can only be an argument for the right to participate and share, as well as for the availability of the condition(s) for participation and sharing. From the perspective of the disengaged self, insofar as every action is an action of and by autonomous, rational beings, there cannot be any obligations for them to participate and share actively. This is, perhaps, best illustrated by putting alongside the idea of sharing and charity: sharing is viewed as an act of charity precisely because there are no obligations for individuals to share. ${ }^{15}$

I think the notion of the reflexive self from Giddens and Beck et al. allows us to make better sense of Shirky's judgement and the attitude (re)presented by it. Recall my discussion of the reflexive self: inherent to the reflexive self is a radicalised reflexivity that challenges certainty and any standardised criteria, and compels individuals to choose actively in accordance to their own will. Accordingly, self-discipline is replaced by self-choice in the reflexive self, which helps to explain why Shirky's plea for the unorganised, the rejection of authority and improvisation can be viewed as plausible. Similarly, the reflexive self's acceptance of fluidity through its challenge of certainty and standardised criteria also helps to explain the fluid worldview presumed in Shirky's judgement. Indeed, as the term 'reflexive' in the reflexive self implies, rational deliberation-the cornerstone of the disengaged self-is no longer prized in the reflexive self, instead what matters is the ability to

\footnotetext{
14 See, Chapter 2, section 2.2.1 and section 2.4.1.

15 Also, see Turkle (2011). Although Turkle's focus is on intimacy, her point is instructive. She notes that in the "[t]raditional views [...] autonomy and strong personal boundaries [are] reliable signs of a successfully maturing self. [...] [W] w work toward an independent self capable of having a feeling, considering it, and deciding whether to share it. Sharing a feeling is a deliberate act, a movement toward intimacy" (Turkle 2011, pp. 174-175; my emphasis).
} 
intuitively and instantaneously cope with the unorganised, ungoverned/unguided and unexpected, i.e. the reflex.

Shirky's imperative to participate and share can be better understood with a reinterpretation via the late modern self, and Beck et al.'s notion of quasi-subject is particularly useful here. To reiterate, the reflexive self, as a quasi-subject, is "a constitutive part of a context that determines its subjectivity, and within which exercises joint decision-making power" (Beck et al. 2003, p. 26). Accordingly, individuals themselves do not exhaustively determine their subjectivity; at the same time, because of the redistribution of their (quasi-)subjectivity over their networks, which, in turn, are connected to others' networks, individuals' decisions and actions become a constitutive parts of others' subjectivity, too. The redistribution of (quasi-)subjectivity over the networks in the late modern self implies that every action is no longer an action only of and by individuals themselves, but of and by the networks they are connected to. So, realisations of any decisions and actions are dependent on others' (quasi-)subjectivity as well. It thus entails a new obligation for them to involve actively in their networks, because they are mutually dependent within the networks. So construed, the imperative to participate and share can be reinterpreted as an explicit formulation of the norm of quasi-subjectivity in the networks.

In short, the different judgements of Web 2.0 (re)presented by Shirky and Keen can be traced back to the different normative and axiological foundations their judgements are based on. For Shirky and the view he represents, the basis of the judgements comes from the reflexive self, which accepts fluidity and uncertainty of any foundations and sees individuals as quasi-subjects who are obliged to involve actively because of the mutual dependence inscribed by the logic of the networks. For Keen and the view he represents, the basis of the judgements comes from the disengaged self, in particular, the idea of subjects as autonomous, rational beings prescribed by it.

\subsection{Vices and Virtues of a Network State of Mind}

Like Keen's The Cult of Amateur and Shirky's Here Comes Everybody and Cognitive Surplus, early prudential appraisals of Web 2.0 mainly focus on Web 2.0's impacts on the society-at-large. However, as Geert Lovink rightly noted, the recent popular discourse on Web 2.0 has taken a "neurological turn" (Lovink 2010). The "neurological turn" of prudential appraisals can be viewed as an interiorisation of 
the concerns over Web 2.0, namely Web 2.0 does not only affect people's exterior life, e.g. their society, it also affects their interior life, i.e. their brain (or mind, selfhood, etc.). In this section, I will examine four representative examples of prudential appraisals that have taken the "neurological turn" and uncover their normative and axiological foundations.

\subsubsection{Cognitively and Emotionally Shallow Minds}

Nicholas Carr's “Is Google Making Us Stupid” (2008) is arguably the first prudential appraisal in the "neurological turn". He has later expanded his idea into the book The Shallow: What the Internet is Doing to Our Brain (Carr 2010). In the book (and the earlier article), Carr laments that the Internet, with its defining characteristics, i.e. interactivity, hyperlinking, searchability and multimedia, has detrimentally affected our habits of reading, writing and thinking. He argues that our ability to have "sustained, unbroken attention to a single, static object" (Carr 2010, p. 64)—a prerequisite for deep reading and deep thinking-has become difficult, if not impossible, with the habitual use of the Internet. According to Carr, reading on screen with scrolling and clicking is phenomenologically different from reading on paper, and the differences between them have a direct impact on our attentiveness. At the same time, he argues that hyperlinking does not merely offer readers supplementary information, but it draws them away from the text they are reading and directs them to other (not so) relevant pieces of information. The same is true for searchability of digitised texts too, or so he argues. Digitised (and thus searchable) texts enable non-linear reading and allow readers to see a text in and as fragments but not as a coherent, unified narrative. Finally, being a simultaneous medium of various types of information, the Internet has further weakened our ability to focus on and attend to the content of a text-if it is still sensible to say that there is the content at all (Carr 2010, pp. 89-92). In short, Carr's criticism is one against a particular kind of mind (or type of cognition) moulded by the Internet, i.e. an inattentive, shallow mind.

Carr's criticism, in effect, is targeted against the notion of the reflexive self. As I have noted, the reflexive self downplays the ability of rational deliberation and prizes the ability to respond intuitively and instantaneously. Here, the ability to skim with scrolling and clicking-instead of immersing oneself in a text through deep reading and deep thinking - and the ability to jump from one place to another with hints of hyperlinks and search results both within and outside a text are clear instances of reflexive actions valued by the reflexive self. Similarly, Carr's worry over the fragmentation of the text is also based on the assumption that fluidity- 
something the reflexive self accepts without qualms-is problematic. ${ }^{16}$ In this respect, Carr's criticism is essentially against the reflexive self, which, in his account, will inevitably be inattentive and shallow.

Still, the question remains: why are deep reading and deep thinking valuable at all? Here, Carr's description of deep reading (and deep thinking) is illuminating,

"What is so remarkable about [reading] was that the deep concentration was combined highly active and efficient deciphering of text and interpretation of meaning, [deep reading] was valuable not just for the knowledge readers acquired from the author's words but for the way those words set off intellectual vibrations within their own mind. [...] [i.e.] people made their own associations, drew their own inferences and analogies, fostered their own ideas." (Carr 2010, pp. 64-65; my emphasis)

What is so valuable about deep reading and deep thinking is that they provide an opportunity for people to exercise their own creativity to formulate thoughts that are uniquely theirs. Accordingly, reading (and thinking) is not only about receiving information and/or knowledge from texts, reading (and thinking) ought to "[inspire] new insights, associations, and perceptions, sometimes even epiphanies" (Carr 2010, p. 74). Carr's foregrounding of creativity, individuality (i.e. thoughts that are unique to the person) and authenticity (i.e. thoughts that belong to them) should be familiar, because they are the basic values embodied in the expressive self. In short, Carr's criticism and the attitude to the Internet it (re)presents are grounded in the expressive self.

Carr's criticism of digital media, as we have seen, focuses only on its impacts on our cognitive capacity and leaves out our emotional capacity. In this respect, Sherry Turkle's Alone Together: Why We Expect More from Technology and Less from Each Other (2011) is an appropriate complement to Carr's criticism of digital media,

\footnotetext{
16 The worry over the fragmentation of the text (or, alternatively, the valuing of the coherency and unity the text) can also be found in Carr's discussion of the Internet's impacts on writing. In The Shallows, he compares printed books with ebooks, and he states that printed books differ from ebooks in that they are finalised. The finality of printed books, he argues, compels authors to perfect their works to ensure that they truly (re)present their own thoughts before they are being published. He argues that this mentality of perfection (and, relatedly, being truthful to oneself) has dissipated in ebooks because ebooks are viewed as a process, i.e. something editable by authors even after they are released (Carr 2010, pp. 107-108).
} 
as it concerns with the impacts of digital media on our emotional capacity. In her discussion, Turkle attempts to show that digital media has bred an equally shallow emotional mind. ${ }^{17}$ And, like Carr, Turkle expresses worry over the loss of richness of the mind, individuality and authenticity with the rise of habitual digital connection. Turkle's criticism of digital media, I think, can too be viewed as being grounded in the expressive self.

Turkle notes that digital media affords a new understanding of distance and time: distance is now defined by connectivity and availability but not by physical space (Turkle 2011, p. 155-156); time has also received a new meaning as digital media allows people to layer multiple activities on a single temporal unit (Turkle 2011, p. 164). The new understanding of distance (or space) and time, in turn, makes possible "life mix", i.e. a new form of life that is "the mash-up off what you have on- and offline" (Turkle 2011, p. 160). "Life mix", in Turkle's view, is dangerous because it causes distraction and a loss of attention (Turkle 2011, Chapter 8 \& 14). Distraction and the loss of attention in "life mix", however, are unlike what Carr has described. They are not cognitive, but emotional. In living in different (onand offline) lives in simultaneity, people's attentions-in terms of emotional involvement and attachment-are distributed across different lives they have simultaneously, and thus attention to each life is inevitably thinned out. The result, therefore, is a loss of richness in emotional involvement and attachment to specific persons and/or situations.

Turkle also voices worry over the changing conditions of personal engagement that come with constant connectivity. Digital media allows young people (and adults) to always stay connected to their parents and peers. Constant connectivity, however, has the side-effect of removing the private space from individuals, which is essential for them in their individual development. In effect, she argues that digital media favours "the collaborative self" of which the self only feels emotionally complete when it is connected (Turkle 2011, pp. 171-179). In short, the (internal) richness of individuals has given way to (external) connectivity in a digitally-mediated life. And, the worry over the rise of "the collaborative self" should sound familiar. Indeed, Turkle's worry compares nicely with Shirky's praise of the imperative of (inter)activity, which is grounded in the idea of quasi-subject.

${ }_{17}$ In Alone Together, Turkle discusses the impacts of both sociable robots and digital media on our emotional capacity. In this section, I only look at Part II of Alone Together (esp. Chapter 8-14), where she voices her concerns over the impacts of digital media on our emotional capacity. (Turkle 2011) 
Like Shirky, Turkle sees an emergence of quasi-subject with the rise of social media. Unlike Shirky, however, she foregrounds the loss of individuality and authenticity from becoming the collaborative self and deems that to be undesirable.

Finally, although "life mix" can expand people's ability to experiment with their identity in their (online) lives, digitally enabled "life mix", paradoxically, can also hamper the authenticity of their (offline) identity. Indeed, Turkle agrees that online worlds allow people to rework their identity and practise new identity in their online lives (see, e.g. Turkle 2011, pp. 179-182, 189-194, 211-224), but she also warns that social media-the new, and now also the most prominent, space for (online) identity construction-is significantly different. In social media, users are compelled to present themselves in a simplified manner (Turkle 2011, pp. 179-186). In this respect, social media also compels us to editing away our authenticity, at least, for our online lives. Yet, authenticity is at stake not only when people are using social media, but it is more so when their (new) identity in online worlds come to live on their own terms and replace their offline identity (Turkle 2011, Chapter 11).

\subsubsection{The Hive Mind}

As Carr and Turkle set to examine how digital media has changed the way we think and feel and whether the new mind arises from those changes is desirable or not, Jaron Lanier examines the impacts of digital media on us from a more general perspective in You Are Not a Gadget: A Manifesto (2010) and discusses how it influences the way we perceive ourselves as human beings. ${ }^{18}$

Lanier sets to criticise Web 2.0 in his book; he argues that the advent of Web 2.0 has led to a "reduced expectation of what a person can be, and of who each person might become" (Lanier 2010, p. 4). And, he argues that the reduced expectation is a result of modelling and designing Web 2.0 on the basis of the hive mind, that is - to see "a random crowd of humans [as] an organism with a legitimate point of view" (Lanier 2010, p. 4). In his view, the current Internet (i.e. Web 2.0) has crystallised the idea of the hive mind by encouraging people to see "the Internet

\footnotetext{
18 Lanier, however, not only discusses the impacts of digital media on our selfinterpretation and self-understanding; he also discusses its impacts on economy (Lanier 2010, Chapter 4-8) and culture (Lanier 2010, Chapter 9-11). But, I will only focus on his view on how Web 2.0 has changed our self-interpretation and self-understanding in this section.
} 
as a whole is [being] alive and [...] a superhuman creature" (Lanier 2010, p. 14). Lanier's fear, accordingly, is that there is, or will be, a replacement of "individual intelligence" by "the pack mentality" (Lanier 2010, p. 5). For him, digital media has quickened and amplified this replacement by heightening the importance of nonhuman and/or non-personal intelligence (of the crowd) and degrading the wholeness of human being and person.

In essence, therefore, Lanier's criticism of Web 2.0 is an argument against antihumanism, i.e. the view that there is nothing unique about human beings in comparison to other entities, and anti-personalism, i.e. the view that there is nothing unique about a specific person in comparison to other persons. Or, in Lanier's own terms, his argument is against what he called "computationalism", i.e. the view that "the world can be understood as a computational process, with people as subprocesses" (Lanier 2010, p. 153). Against anti-humanism, anti-personalism and computationalism, Lanier curiously tries to revive the uniqueness of humanity and personhood by imbuing certain mystical qualities on human beings and beinga-person. For instance, he argues that the value of information necessarily rests on personal experience (Lanier 2010, pp. 28-29); and, he also argues that human consciousness is uniquely human (as a personal phenomenon) that cannot be replicated through computation or found in the hive mind (Lanier 2010, pp. 29-44, 153-157). In doing so, Lanier attempts to reassert the primacy of humanity and personhood against the non-human and the impersonal.

For Lanier, the hive mind (or, dehumanisation, depersonalisation and computationalism) is problematic because he believes that they will all incur a loss of personal creativity, as people are no longer "being treated as the source of their own creativity" (Lanier 2010, p. 16; my emphasis; also see, Lanier 2010, Chapter 9). At the same time, he also believes that they will incur a loss of diversity by encouraging and compelling people to tailor themselves to the crowd and disregard the personal flavours (Lanier 2010, Chapter 10).

Now, it should be relatively clear that Lanier's criticism of Web 2.0, like Carr's and Turkle's, is also grounded in the expressive self. His criticism foregrounds the importance of individuality and the evils of the hive mind (or, dehumanisation, depersonalisation and computationalism), i.e. the loss of individuality and authenticity, especially in terms of the wholeness of human being and person. He also emphasises the significance of (artistic) creativity and the importance of seeing creativity as a personal quality. Yet, the most telling part of Lanier's criticism is his attempt to imbue mystical qualities on human being and personhood. Of course, this does nothing by itself tell us what is the normative and axiological foundation 
of Lanier's criticism, but it does imply that his criticism is not based on the idea of the subjects as autonomous, rational beings.

\subsubsection{Minds of the Future}

Prudential appraisals in the neurological turn are mostly negative. This is predictable because an alteration of our cognitive and emotional mind and of who we are seems to pose a greater and more immediate danger than alterations of our surroundings. Still, it is possible to find a few prudential appraisals that hold a less hostile and more positive attitude towards the (potential) changes in our brain (or mind, selfhood, etc.). One example is Nick Bilton's I Live in the Future \& Here's How It Works: Why Your World, Work, and Brain Are Being Creatively Disrupted (2010). Bilton's view is particularly interesting because he himself belongs to the group of people who grew up with digital media, i.e. the digital natives, and his view is presumably articulated from this perspective (Shafer 2010). In this respect, Bilton's judgement of digital media can be seen a representation of the view held by digital natives, who take the impacts of digital media not so much as changes but more so as a given.

As the subtitle of Bilton's book suggests, his discussion is not only limited to digital media's impacts on our brain. Yet, I will only focus on Bilton's discussion of the new state of mind emerging from the use of digital media, which, in turn, can be seen as a response to the pessimistic attitudes held by Carr, Turkle, Lanier and the like. A contrast between Bilton's (more) positive view and the pessimistic views previously analysed will help to illuminate the different normative and axiological foundations of the two sides.

Bilton has responded to the worry raised by Carr over the possibility of distraction and the loss of attention caused by the habitual use of the Internet. In his response to Carr, Bilton accepts Carr's ideas that (i) reading on screen (or surfing the Internet) and reading on paper are phenomenologically different, and (ii) that the Internet, because of those characteristics identified by Carr, are distracting and causing a loss of attention, and, finally, (iii) that deep reading and deep thinking as Carr has characterised are unlikely to happen in an online environment (Bilton 2010, Chapter 5). ${ }^{19}$ However, Bilton argues that none of those changes should be

\footnotetext{
19 It is not entirely true to claim that Bilton believes deep thinking cannot happen in an online environment, as he states that "[s]ome say the Internet and multitasking with media are making our brains into one big distraction zone incapable of handling complex
} 
viewed as devastative to us. He argues that there are other cognitive skills, e.g. multi-tasking, which are equally—or, even, more-important, and deep reading and deep thinking are just one of the many cognitive skills available to us (Bilton 2010, Chapter 7). He writes matter-of-factly that the shift to multi-tasking is natural because it is merely a (new) mechanism to cope with the changing nature of our information environment, which is analogous to people's learning of (deep) reading for acquiring information in books and long-form narratives in the past (Bilton 2010, Chapter 7). He holds the same view towards social media (and thus the hive mind), too. Unlike Lanier, who sees the hive mind as problematic, Bilton takes the wisdom of the crowd made possible by social media merely to be a (new) mechanism to cope with information (over-)abundance, which, according to Bilton, is simply a given for him and the digital natives (Bilton 2010, Chapter 3).

Although Bilton does not make explicit what is of value about digital media, his rebuttal to Carr's criticism and Lanier's criticism is instructive. He sees digital media to have provided people new mechanisms to cope with the new fast-paced and information-rich environment, which requires rapid and nimble responses from individuals. This aligns Bilton's judgement of digital media fittingly with the reflexive self, which stresses the importance of individuals' (cognitive) capabilities to construct their self-narratives via lifestyle choices and life-planning. Indeed, in Bilton's account, these new mechanisms are not to be afraid of, but are to be championed, because they are only a "constructive way [for ourselves and our brain to adapt] to this new online world" (Bilton 2010, p. 136; my emphasis). In other words, multitasking and the wisdom of the crowd are desirable for people because they are seen as essential to the construction of self-narratives in a digitallymediated world.

Before moving to the next section, I should mention that Section 3.2 and Section 3.3 do not intend to suggest which of the prudential appraisals are more satisfactory (or, which attitudes towards digital media and digitally-mediated practice we ought to hold). The aim of my philosophical (re)interpretation is only intended to uncover the normative and axiological foundation of the selected prudential appraisals, and

ideas or long-form narrative. I don't agree" (Bilton 2010, p. 197; my emphasis). But, it is helpful to point out that what he means by 'complex ideas' and 'long-form narrative' are very different from what is described by Carr. Particularly, his ideas of 'complex ideas' and 'long-form narrative' appear to be about multiple and constant sensual stimulations. 
thus to reveal why and how digital media and digitally-mediated practices are judged by the people in certain ways. In other words, my focus has only been on the value dimension of prudential appraisals. In order to decide which prudential appraisals offer a more satisfactory assessment of digital media and digitally mediated practices, it is necessary to also examine their empirical dimension and conceptual dimension as well. ${ }^{20}$ Although the foregoing discussion does not tell us which prudential appraisals are better, or what our normative judgements on digital media and digitally-mediated practices ought to be, I think it does enable us to make better normative judgements on digital media and digitally-mediated practices, as it informs us how our normative judgements should be made, or so I shall argue in the next section.

\subsection{Who Should We Be in a Digitally-Mediated World?}

My analysis of prudential appraisals in Section 3.2 and Section 3.3 is by no means an exhaustive representation of the notions of the self and the embodied ideals (and the views of the good life) in all normative judgements on digital media and digitally-mediated practices, but I think my (re)interpretation of the representative examples of prudential appraisals has illustrate the role of the self (i.e. the disengaged self, the expressive self and the reflexive self) in grounding different normative judgements on digital media and digitally-mediated practices, and, in turn, has provided strong support to the claim that the self is the normative and axiological foundation for determining the issues pertaining the relations between digital media and the good life. This, I think, has an important implication to normative analysis of digital media and the good life, as well as our own normative judgements on digital media and digitally-mediated practices, because it entails any normative judgements on the relations between digital media and the good life cannot be made without also invoking some notions of the self, even if we have already been doing so implicitly.

Accordingly, a specific pattern of normative judgments on the relations between digital media and the good life, which can often be found in both scholarly

20 For a discussion of the three dimensions of prudential appraisals, and how we can assess them, see Chapter 1, section 1.4.2. 
and popular discourse, will necessarily be incomplete and insufficient. The pattern of normative judgements $I$ have in mind has the following form: ${ }^{21}$

(J1i) Digital media T promotes some values V1...n.

(J2i) V1...n are bad/undesirable (for individuals) morally and/or prudentially.

(JCi) $\mathrm{T}$ is bad/undesirable (for individuals) morally and/or prudentially. Or,

(J1ii) Digital media T demotes some values V1...n.

(J2ii) V1...n are good/desirable (for individuals) morally and/or prudentially.

(JCii) $\mathrm{T}$ is bad/undesirable (for individuals) morally and/or prudentially. ${ }^{22}$

This pattern of normative judgements can be found, for example, in Keen's and Carr's criticism of digital media. For instance, Keen argues that Web 2.0 is bad (i.e. (JC)), because it promotes amateurism (i.e. (J1)), and he regards amateurism to be undesirable (i.e. (J2)). Similarly, Carr argues that the habitual use of the Internet reduces our capacity to focus and concentrate (i.e. (J1)), and he sees this capacity to be essential for a good life (i.e. (J2)). So, he concludes that the habitual use of the Internet is bad (i.e. (JC)).

Here, my claim is not that digital media (and digitally-mediated practices) does not promote or demote certain values, or that digital media is neutral. We have

\footnotetext{
${ }^{21}$ It is a simplified formulation of the pattern of normative judgements. Particularly, it is worth pointing out that $\mathrm{T}$ can promotes (or demotes) $\mathrm{V}_{1 \ldots \mathrm{n}}$ indirectly through encouraging (or discouraging) some behaviours and practices $U_{1 \ldots}$ that promote (or demote) $V_{1} \ldots n$. Since this addition does not affect my criticism of this pattern, I shall use the simplified formulation to avoid unnecessary complication.

22 This pattern, of course, can also be formulated differently to argue in favour of digital media. Accordingly, the pattern of normative judgements will be the following:

$\left(\mathrm{J}_{1 \text { iii }}\right.$ Digital media $T$ promotes some values $\mathrm{V}_{1} \ldots \mathrm{n}$.

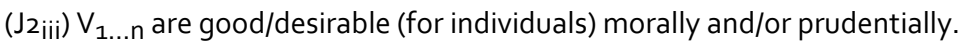

$\left(\mathrm{JC}_{\mathrm{iii}}\right) \mathrm{T}$ is good/desirable (for individuals) morally and/or prudentially. Or,

$\left(\mathrm{J}_{1_{\text {iv }}}\right)$ Digital media $T$ demotes some values $\mathrm{V}_{1 \ldots .}$.

$\left(\mathrm{J}_{2 \mathrm{iv}}\right) \mathrm{V}_{1 \ldots \mathrm{n}}$ are bad/undesirable (for individuals) morally and/or prudentially.

$\left(\mathrm{JC}_{\mathrm{iv}}\right) \mathrm{T}$ is good/desirable (for individuals) morally and/or prudentially.

Regardless of the formulation, my criticism applies so long as the pattern of normative judgements in question does not make explicit its normative and axiological foundation, namely what makes the values $V_{1} \ldots n$ valuable.
} 
already seen with sufficient evidence that digital media does promote or demote certain values. It is also not my intention to contest the goodness (or badness) of specific values promoted (or demoted) by digital media. My focus here is on the form of this pattern of normative judgements, and my claim is that even if (J1) and (J2) are true and (JC) does follow, normative judgements with this pattern are always incomplete and insufficient because they fail to acknowledge (or, at least, make explicit) the fact that they are grounded in a specific notion of the self. Since, as I have argued in Chapter One, it is various notions of the self that inform us what is of value. This, ultimately, also renders normative judgements with this pattern indecisive and ineffective, because without settling on a specific view of the self, the discussants are only affirming their own views of what is of value and what is a good life and ignoring the possibility that their views may not be shared by others. In other words, they are (or, at least, risk) talking passed the others.

If my argument against the completeness and sufficiency, and thus the decisiveness and effectiveness, of this pattern of normative judgements is sound, then we have to examine various notions of the self more carefully before we can meaningfully engage with the values and disvalues of digital media. After all, when what is of value is in question, merely insisting something is of value will not take us very far. Particularly, as I have attempted to show in the previous sections, the positive and negative attitudes towards digital media appear to be originated from different notions of the self: those who hold the modern notions of the self tend to judge digital media and digitally-mediated practices negatively, and those who hold the late modern notion of the self tend to judge them positively. If it is indeed so, instead of (only) arguing for or against digital media on the basis of the values it promotes or demotes, we should first examine why those values are of value and whether they are still of value by redirecting our attention to the question: which notion(s) of the self we should strive for in our digitally-mediated world? Or, in other words, who should we be in our digitally-mediated world? ${ }^{23}$

This shift of attention to the question concerning who we should be is also significant to our pursuit of an informed public discourse on digital media and digitally-mediated practices as well. Particularly, as our normative judgements are based on the notion(s) of the self we hold, our public discourse cannot afford to

\footnotetext{
23 Cf. Winner (1996). Winner arrives at the same conclusion from a different argument. In his article, Winner argues against the focus on the use of information technology, i.e. "what to do [with information technology]", and urged researchers to refocus on the question on "who to be [with information technology]" (Winner 1996, p. 71).
} 
ignore the different notions of the self, if we want it to be a genuinely informed public discourse. At the same time, when researchers and critics are recommending certain human-digital media relations to the public, their recommendations can be reinforced by clearly showing why those human-digital media relations should be held by the public, i.e. by showing those human-digital media relations are prescribed (or, at least, favoured) by the notion(s) of the self the people themselves maintain. Alternatively, by showing why and how some notions of the self cannot adequately answer the question of 'who we should be in the digitally-mediated world', we can also demonstrate to the public why and how certain normative judgements on digital media and digitally-mediated practices are wrongheaded, as well as convince them to follow our recommendations.

Unfortunately, I do not think there is a definitive answer to the question of 'who we should be in our digitally-mediate world'. However, my (re)interpretation of prudential appraisals in Section 3.2 and Section 3.3 should have at least served to make explicit various notions of the self lie underneath the positive and negative attitudes towards digital media and digitally-mediated practices, and thus it should enable us to better assess them by showing explicitly why digital media and digitally-mediated practices are of value. For example, traditional cultural institutions and experts (in Keen's criticism), participation and sharing (in Shirky's account), deep reading and deep thinking (in Carr's criticism), emotional involvement and attachment (in Turkle's criticism), the wholeness of humanity and personhood (in Lanier's criticism) and multi-tasking and the wisdom of the crowd (in Bilton's account) are of value because they are constitutive of specific ways of self-interpretation and self-understanding. Disclosing the normative and axiological foundation of prudential appraisals, i.e. the self, should also allow us to better judge digital media and digitally-mediated practices, because it can advance our own normative judgements on the relations between digital media and the good life by opening up the space for a reappropriation and fusion of various notions of the self.

Yet, the hard question of 'who should we be in our digitally-mediated world?' remains. In the remainder of this chapter, I will try to shed light on the answer(s) to this hard question. However, as I have previously noted, I do not think a definitive answer can be (easily) given. So, instead of giving an answer to the question, I will only consider how we might answer it. 


\subsubsection{Ess on Digital Media and the Self}

In Chapter One, I have argued that our self-interpretation and self-understanding are socially and culturally conditioned; and, in my exegesis of the modern and late modern self through the works of Taylor, Giddens and Beck et al., I have shown how new notions of the self emerge from new social, cultural and historical backgrounds. Now, I want to add that (digital) media is part of the background for our self-understanding and self-interpretation as well (see, e.g. Ess 2010, 2011; Bakardjieva \& Gaden 2011). If this is so, understanding the interactions between digital media and the self is paramount for our answering of the question of 'who should we be in our digitally-mediated world?', because digital media too (co-)shapes our self-interpretation and self-understanding and (dis)favours certain notions of the self.

In his recent works, Charles Ess (2010; see also Ess 2011) has systematically explored the interactions between digital media and the self. Drawing from communication theory, information ethics and phenomenology, he argues that different modalities of communication (i.e. orality, literacy, print and secondary orality of digital media), together with the different values and skill sets associated with them, are strongly correlated with different ways of self-interpretation and self-understanding. In a nutshell, he points out that literacy and print are strongly correlated to an individualistic understanding of the self (Ess 2010, pp. 106-108), whereas secondary orality of digital media is strongly correlated to a distributed (or relational) understanding of the self (Ess 2010, pp. 110-111). More specifically, Ess conceptualises the self arising from secondary orality of digital media with the idea of "smeared-out self", i.e. "[a self] distributed across CMC networks via multiple means of communication (SMS, social networking sites, micro-blogging, email, etc.) that thereby represent hundreds, if not thousands, of simultaneous but potential relationships/engagements that [individuals] realise one at time" (Ess 2010, p. 111).

Ess's analysis of the interactions between digital media and the self is instructive, as it illustrates how digital media can affect our self-interpretation and self-understanding by enabling (or, indeed, compelling) individuals to move away from an individualistic understanding of the self, which are characteristic of the modern notions of the self (i.e. the disengaged self and the expressive self) and (re)turn to a distributed/relational understanding of the self, which are more characteristic of the self in late modern societies. Indeed, it is plausible to conclude from this that digital media actually favours the late modern self and disfavours the modern self. Recognising this dynamic is important, as it provides hints of why certain arguments for (or against) digital media and digitally-mediated practices 
become (un)persuasive, e.g. Shirky's imperative of participation and sharing is persuasive because it adheres to an understanding of the self that is favoured by digital media. This, in turn, should make us aware of the (un)persuasiveness of the arguments actually come with the rise of digital media, and thus they should not be taken for granted.

More importantly, Ess argues that it is the values (and skill sets) of the modern notions of the self, especially of the disengaged self (e.g. knowledge, autonomy, equality, impartiality and tolerance), that buttress the idea of democracy. He notes that without the values (and skill sets) of the modern notions of the self enabled by literacy and print, there is a risk of (re)turning to authoritarianism. In response to this risk, Ess proposes we should see secondary orality of digital media not as a replacement of literacy and print, but only as a compliment to them. It is not my intention to argue for or against Ess's proposal here, but his proposal has provided us an important insight to answering the question of 'who should we be in our digitally-mediated world?', that is-the answer to this question is not merely personal because it also depends on the type(s) of society we want to live in. For instance, if we want to live in a democratic society, which, according to Ess, is strongly correlated to (or, in effect, only made possible by) the values and skill sets of the modern self (e.g. the disengaged self), then we must somehow be able to incorporate (the values and skill sets of) the modern self in our answering of the question. Yet, to realise the type(s) of society we want to live in is not merely a personal matter, it is also a socio-political matter too. In short, our idea(s) of society also contribute to our answering of the question as well.

\subsubsection{In Pursuit of the Self in a Digitally-Mediated World}

Ess's analysis nicely shows that an answer to the question of 'who we should be in our digitally media world?' requires us to reflect on the type(s) of society we want to live in. There is, however, another lesson from Ess's analysis of the interactions between digital media and the self: if digital media does interact with the self and (dis)favours certain notions of the self, then there is also a need to (re-)examine to what extent various notions of the self are still plausible with the rise of digital media. Or, to put it differently, we must (re-)examine how the rise of digital media affects the options we have for answering the question.

Recently, Mark Deuze (2010, 2011, forthcoming) has argued for an ontological turn in (digital) media studies. He argues that digital media has become so pervasive and ubiquitous to the extent that it has also become the building blocks of our experience in everyday life and the structure of our meaningfulness. Deuze's claim 
is not only that we encounter and use a variety of digital media in our everyday life, but that we cannot experience our everyday life and make sense of ourselves and the world around us without them. In other word, Deuze's claim is that digital media can no longer be seen as something extrinsic to our experience and sensemaking, but it must be taken as intrinsic to them. Accordingly, digital media is a constitutive part of our ontology, as Deuze has put it, "we lived in, rather than with, media-we are living a media life" (Deuze 2011, p. 138; see also, Deuze forthcoming). Of course, Deuze's attempt to (re)conceptualise (digital) media as a constitutive part of our ontology is not uncontroversial and requires further scrutiny. However, if Deuze is right, then it entails digital media is at the same time constitutive of our self-interpretation and self-understanding, too; since we must then always interpret and understand ourselves and the world around us through digital media. ${ }^{24}$

So construed, and if Deuze is right, any plausible answers to the question of 'who we should be in our digitally-mediated world?' must make room for a constitutive relation between digital media and the self. In other words, they must be able to account for the role of digital media as a constitutive part of our selfinterpretation and self-understanding. This requirement, in turn, might rule out some notions of the self as plausible answers to the question.

For instance, the modern notions of the self (e.g. the disengaged self and the expressive self) appear to be particularly at stake. As Peter-Paul Verbeek (2009) has convincingly argued in the field of philosophy and ethics of technology, the modern notions of the self distinguish too sharply between the subject and objects (and between persons and things) ontologically, and that the rigid ontological separation of the subject from objects (and persons from things) inevitably bars them from taking objects and things to be morally significant, because morality is then confined entirely to a specific ontological domain, i.e. the subject's domain, where objects and things are excluded entirely. Although Verbeek's discussion

\footnotetext{
24 Although Ess's analysis shows that (digital) media does interact with the self, it is less clear if Ess is going to accept Deuze's more radical conclusion that digital media is constitutive of the self. Indeed, there are two ways to conceive the interactions between digital media and the self, namely (i) it can be viewed as instrumental for the (be)coming of a specific notion of the self, e.g. literacy and print enable the rise of an individualistic understanding of the self, and secondary orality of digital media enables the rise of a distributed/relational understanding of the self, and (ii) it can be viewed as constitutive of a specific notion of the self, as Deuze argues.
} 
mainly focuses on the morality of objects and things, his argument against the incapability of the modern notions of the self to take objects and things to be morally significant can be easily translated into the present context: if the modern notions of the self draw a sharp boundary between the self (i.e. the subject) and digital media (i.e. the object), and they are seen as belonging to two radically distinct ontological domains, then digital media cannot be taken as constitutive of the self because of their ontological differences. In this respect, the modern notions of the self-or, for that matter, any notions of the self that presume a sharp ontological difference between the subject and objects (and persons and things) cannot provide a plausible answer to the question of 'who we should be in our digitally-mediated world?'. ${ }^{25}$

The implausibility of the modern notions of the self as answers to the question, however, seemingly presents us with a predicament: (i) even if we see the criticisms of digital media and digitally-mediated practices arrived from modern notions of the self as real, e.g. the devalue of scientific standards and scientific rationality, the loss of deep reading, deep thinking, and deep emotional involvement and attachment, etc., the modern notions of the self have lost their normative grounds to (re)assert their importance. Alternatively, (ii) consider Ess's proposal for preserving the values and skill sets of the modern notions of the self. It seems to be difficult, if not impossible, to do so if the modern notions of the self cease to be a plausible answer to the question.

Where, then, does this predicament lead us to? Does it mean that we must happily accept the 'loss' of the values and skill sets of the modern notions of the self, and see it as an emancipation from them? Here, it is helpful to remind ourselves that the self is not simply fixed. Indeed, the exercise of philosophical (re)interpretation I have performed in Section 3.2 and Section 3.3 has not only

\footnotetext{
25 To overcome "the humanist bias of ethics" (Verbeek 2009, pp 245-248), Verbeek argues for an ethics of design and ethics of technological mediation, where the former examines design as a practice of materialising morality, the latter examines the moral nature of technological mediation (Verbeek 2009, pp. 255-260).

Although I am generally sympathetic to Verbeek's approach, I worry that his emphasis on materiality and technological mediation in lieu of the language of morality has an unintended consequence of cutting the public off from moral deliberation. Particularly, we should be aware of the fact that (ethics of) design is monopolised by those who are involved in the processes of design, and it is not open to the public. See, Chapter 6 for a further discussion.
} 
shown how various notions of the self are at work in our normative judgements of digital media and digitally-mediated practices. At the same time, it has also uncover the normative and evaluative resources we can (re)appropriate in our pursuit of the notion(s) of the self that might serve as a plausible answer to the question. In other words, even if the modern notions of the self alone are no longer plausible as an answer to the question of 'who we should be in our digitally-mediated world?', it does not by itself rule out the possibility of creative (re)appropriation and fusion of the values and skill sets they embody. However, with the continuing development of digital media, I contend that the question will remain as an open question that affords no definitive answer. Yet, the key to answering this question, I think, lies in our effort to disclose various notions of the self and to creatively (re)appropriate and fuse them.

Still, it is useful to remind ourselves of the socio-political dimension of the question of 'who we should be in our digitally-mediated world', because it provides us a point of critical reflection in our answering of it. By acknowledging the fact that our answers to the question depends on the type(s) of society we want to live, it essentially limits our answers to the question, as it specifies the values and skill sets we need to have in order to realise that type(s) of society, and thus informs us which notion(s) of the self we ought to strive for, or who we should be.

Finally, this discussion cannot be complete without also briefly mentioning the role played the government and policy-makers in shaping our digitallymediated world through various agendas and policies on the development and diffusion of digital media in our society, as the government and policy-makers are essentially promoting some ideals through their agendas and policies. If we, i.e. the people, are to answer the question of who we should be ourselves, instead of having answers imposed on us, then the government and policy-makers ought to open up their agenda and policies for public discussion. Here, I am not suggesting the government and policy-makers must not hold any ideals in their agendas and policies, which I believe is impossible, but they ought to be transparent with the ideals behind their agendas and policies, because it is only then the people can truthfully assess the agendas and policies and compare them with their own ideals. Moreover, it is also important for the government, policy-makers and the people to realise that the question cannot be satisfactorily answer without also taking into account the question about the type(s) of society we are living in (as well as we want to live in). In other words, there is also the need to (re-)establish the sociopolitical dimension of the question and bring it into our discussion on digital media and the good life. 


\subsection{Conclusion}

The main objective of this chapter is to offer a better understanding of the normative and axiological foundation of prudential appraisals by means of a philosophical (re)interpretation of several representative examples. In Section 3.2, I have examined the prudential appraisals that discuss the impacts of digital media on society-at-large (i.e. Keen's The Cult of Amateur and Shirky's Here Comes Everybody and Cognitive Surplus); and, in Section 3.3, I have examined the prudential appraisals that discuss the impacts of digital media on our brain, mind and selfhood (i.e. Carr's The Shallow, Turkle's Alone Together, Lanier's You Are Not a Gadget and Bilton's I Live in the Future \& Here's How It Works). Throughout my analysis, I have attempted to show that they are ultimately grounded in various notions of the self I have identified in Chapter Two.

Acknowledging the self as the normative and axiological foundation of prudential appraisals and our own normative judgements on digital media and digitally-mediated practices means that we should make explicit the notion(s) of the self in prudential appraisals and in our own normative judgements. We have to make explicit the notion(s) of the self in prudential appraisals if we are to properly assess and engage with them. And, we have to make explicit the notion(s) of the self in our own normative judgements, because, otherwise, they will always be incomplete and insufficient, and thus indecisive and ineffective. Moreover, accepting the self as the normative and axiological foundation of our normative judgements on digital media and digitally-mediated practices also means that we should redirect our attention from the (dis)values of digital media to the question of 'who we should be in our digitally-mediated world?', because merely talking about the (dis)values of digital media will bound to be inadequate without at the same time why showing why the values are of value at all. In other words, this chapter has shown that normative analysis of digital media and the good life must begin with the questions about the self, especially the questions about who we should be and who we can be in a digitally-mediated world.

Finally, I end this chapter by looking at what it takes to answer the question of 'who we should be in our digitally-mediated world?'. Although I cannot offer a definitive answer to this question, I have outlined two considerations for any attempts to answer to it. Firstly, I note that this question cannot only be taken as a personal question, because our answers to it depend crucially on the type(s) of society we want to live in. This question, therefore, is also a socio-political question. 
Secondly, I note that recent research in (digital) media theory and philosophy and ethics of technology have convincingly argued that digital media is (or has become) a constitutive part of our self-interpretation and self-understanding. If this line of thinking is right, then our, then our answer to the question must make room for a constitutive role of digital media in our self-interpretation and self-understanding. Accordingly, some notions of the self (e.g. the modern notions of the self, such as the disengaged self and the expressive self) will inevitably fall short as an answer to the question. Yet, by itself, it does not means that we cannot creatively reappropriate and fuse the ideals (and the values) in different notions of the self I have previously identified, but it does means that we need to pay more attention to the kinds of the self we should be in our digitally-mediated world. In other words, we need to (re)open the question of 'who we should be' in normative analysis of digital media and the good life. 


\title{
CHAPTER FOUR
}

\author{
Another Look at the Good Life \\ From a Confucian Point of View
}

In Chapter Two, I have illustrated the views of the good life in the West through Taylor's account of modern identity and the present day ramifications of selfidentity in the writings of Giddens and Beck et al. Although the views of the good life I have summarised in the foregoing discussion provide the normative and evaluative resources for my analysis of prudential appraisals in Chapter Three, they are only applicable to a limited number of discourses on the impacts of digital media and digitally-mediated practices on the good life. For the views of the good life I have explored so far have their roots in the Enlightenment and Romanticism, it is questionable whether those views of the good life can be readily applied to a philosophical (re)interpretation of discourses on digital media and digitallymediated practices in non-Western contexts. In other words, they are inadequate for normative analysis of digital media and the good life in intercultural contexts, or so I argue. Since the development and use of digital media are not only restricted to Europe and North America, a (more) comprehensive normative analysis of digital media and the good life need to examine its impacts in intercultural contexts as well. The main objective of this chapter is to articulating an alternative view of the good life (i.e. the Confucian view of the good life) and thus provide the normative and evaluative resources to examine the relations between digital media and the good life in a different cultural context (i.e. the Chinese context). In doing so, this chapter also aims to make a case for the importance and relevance of non-Western 
notions of the self in normative analysis of digital media and the good life, and to call for a broadening of the scope of normative analysis.

In this chapter, I elaborate the view of the good life in Chinese Confucianism. My choice of Confucianism is theoretical, empirical and personal in nature. Theoretically, Confucianism is one of the most important value systems in our time, and it is also one of the well-developed traditions. In other words, Confucianism offers plenty of resources to arrive at a promising non-Euro/Ameri-centric view of the good life. And, empirically, the Chinese government (i.e. Chinese Communist Party (CCP)) has maintained a purportedly distinct view of digital media and digitally-mediated practices and of their impacts on people's well-being. Their view, as I shall later argue, has its roots in Confucian values. ${ }^{1}$ Looking at Confucianism, therefore, provides with us an interesting point of contrast for the Western and non-Western perspectives. Finally, I come from a place where the influence of Confucianism remains steadily in place. In this respect, Confucianism is a better candidate for reflection than other traditions with which I am unacquainted. In other words, my focus on Confucianism is not to deny the significance of other well-developed traditions, e.g. Buddhism, Hinduism, Judaism, etc. Instead, it is merely a shortcoming on my part.

Before proceeding to the discussion of the Confucian view of the good life, however, I shall first return to the discussion of modernity in Section 4.1. A detour to modernity is necessary because early research on modernity tends to reject the non-West as a proper subject matter. Only recently, has there been a shift towards the idea of plural modernities which try to take seriously the non-West in theorising about modernity. Yet, the discussion of plural modernities is not entirely clear, as similar but different terminologies are being used in various approaches. In that section, I outline, in my mind, the most suitable way to discuss plural modernities for the present study. After outlining my account of plural modernities, I discuss the basics of Confucian ethics and compare them with typical Western ethical theories in Section 4.2 and Section 4.3. The purpose of this discussion is twofold. First, the differences between Confucian and typical Western accounts of ethics in themselves offer an additional reason to go beyond the West in thinking about the issues related to the good life. Second, the discussion provides the basic vocabulary and core concepts for my subsequent analysis of the relations between digital media and the good life in the Chinese context. Finally, in Section 4.4, I

1 See, Chapter 5. 
elaborate the Confucian view of the good life with a special reference to contemporary Chinese culture. Since the objective of this chapter is to illustrate a living view of the good life in Confucianism, it must take into account the social, cultural and historical conditions in which it evolves. Here, I shall not attempt to provide a complete picture of the Confucian view of the good life, but only to highlight the values, especially those that are about the good life, central to contemporary Confucian societies.

\subsection{From Singular Modernity to Plural Modernities}

In early research, modernity (and modernisation) is considered to be a Western phenomenon exclusively. According to this view, there can only be one way to be modern, and there will only be one value system that matters ultimately, because the non-Western, traditional values will eventually give way to the Western, modern ones. ${ }^{2}$ So construed, the study of non-Western, traditional values is, at best, for historical interest. Recent research, however, has started to recognise the diversities and variations of modernity and (re)conceptualise it in plural terms. The shift from singular modernity to plural modernities is significant because it acknowledges not only the importance and legitimacy of value systems in nonWestern cultures, it also highlights the fact that these value systems are also compatible with modernity. This, in turn, allows for a more positive attitude towards the non-Western, traditional values and their possible contribution to $a$ path to modernity (or modernisation). Theorising modernity in plural terms has opened up space for various modernities and, relatedly, other forms of modern identity. Since people's normative judgements are based on their self-interpretation and self-understanding, it also implies that their normative judgements can possibly be radically different too.

Here, I first review the arguments against theorising modernity in singular terms. And then, I outline a framework for the idea of plural modernities. This framework should at least specify how plural modernities can be (and should be) investigated; it should also enable us to talk about different modernities and different forms of modern identity properly.

\footnotetext{
2 This sentiment is, perhaps, best exemplified by Fukuyama's (1992) thesis on "the end of history" and Huntington's (1996) idea of "the clash of civilizations".
} 


\subsubsection{Deficits of Singular Modernity}

Theorising modernity in singular terms has been criticised by philosophers, sociologists, and cultural theorists the like. ${ }^{3}$ Two broad lines of criticisms can be identified in their critiques of singular modernity. I shall call the first line of criticisms the challenge from theoretical and empirical deficits, as it attempts to demonstrate the failure of theorising modernity in singular terms by referring to its unwarranted assumptions and/or empirical counter-evidence. The second line of criticisms focuses on the objectionable moral and political implications of singular modernity. Hence, I shall call it the challenge from normative deficits.

To illustrate the challenge from theoretical and empirical deficits, it is helpful to first expound what theorising modernity in singular terms involves. ${ }^{4}$ There are numerous approaches to singular modernity, but, as Taylor notes, they tend to identify modernity with "some cultural-neutral operation, which is not defined in terms of specific cultures it carries us from and to, but is rather seen as of a type that any traditional culture could undergo" (Taylor 1999, pp. 154-155). Accordingly, modernity (or modernisation) is homogenous, i.e. it consists specific processes of unification and standardisation modelled upon Western cultures. Moreover, they typically accept a strict dichotomy between 'traditional' and 'modern', where the latter is identifiable by a combination of '-isms' and '-isations', which often includes industrialisation, secularisation, capitalism and bureaucratisation. ${ }^{5}$ This, in turn, entails modernity is a static condition.

Some have objected to these assumptions on the basis that these assumptions have neglected the complexities even within Western modernity itself. For example, Venn and Featherstone (2006) have pointed out that many societies that are considered to be paradigmatically modern do not possess many characteristicsthe '-isms' and '-isations' - of modernity. They have also pointed out that alternative narratives of (Western) modernity are readily available (Venn and Featherstone 2006; also see Gaonkar 1999). Their arguments cast doubt on the validity of both the assumptions that modernity is homogenous and that there are characteristics intrinsic to modernity, and thus a clear separation between 'traditional' and 'modern'. Likewise, Peter Wagner (see, e.g. Wagner 1994, 2009),

3 For example, see Taylor (1999) and Bhambra (2007).

4 For an overview of the early works on modernity, which conceptualise modernity in singular terms, see Martinelli (2005, Chapter 2 \& 3) and Delanty (2006).

5 Cf. Oommen (1999), Venn \& Featherstone (2006). 
among others, has convincingly argued that (Western) modernity is best understood in terms of its inherent tensions and contradictions, which entails that modernity is not static but constantly in flux. ${ }^{6}$ In short, many researchers have demonstrated that the idea of modernity is itself contested, therefore, singular modernity, by seeing modernity as homogenous, identifiable by a specific set of characteristics and/or static, is theoretically naïve at best.

Empirical counter-evidence has also disfavoured singular modernity. Modernity, theorised in singular terms, implies that a society must undergo all specific processes for it to be modern. It also implies that the local, traditional values will give way to the modern ones, and thus will eventually arrive at a point of convergence. Yet, neither of the implied consequences has occurred. For instance, many (post-)communist countries are technologically modern, i.e. they are equivalent to many European and North American countries technologically, but are different from them socially and politically. Similarly, East Asia is economically comparable to Europe and North America, but still differs from them in many aspects, especially in terms of social and cultural practices. Also, many socalled 'anti-modern' Islamic countries have succeeded in partial and selective adaptation of characteristics of modernity, e.g. modern technologies (Oommen 1999). The empirical evidence shows that modernity is not all-or-nothing and, in effect, that modernity has more than one dimension-both of which have been neglected in the idea of singular modernity. Moreover, as many researchers have observed, the expansion of (Western) modernity via globalisation has not led to a convergence of cultures. Instead, it has resulted in localisation of global practices, i.e. glocalisation. In response to this observation, some have even suggested that there is not a globalisation but "many globalisations" (e.g. Berger \& Huntington 2003). The same goes for modernity as well. To sum up, there are enough arguments, either theoretical or empirical, against the idea of singular modernity.

Yet, singular modernity is not only objected to theoretically and empirically. It is also objected to on moral and political grounds. The challenge from normative deficits is primarily associated with feminist and post-colonial critics, who argue that the narrative of singular modernity is written by and for (white) men who, at

$6 \quad$ Also, see Ballantype (2008). 
the same time, are also the (ex-) colonisers. ${ }^{7}$ It is, in other words, constructed out of the experiences and histories of the power holders (i.e. (white) men and $\left(e x^{-}\right)$colonisers), and the experiences and histories of the Others (e.g. the nonwhite, non-men, and/or colonised) are not, and cannot be, fully recognised. Hence, singular modernity is exclusive, i.e. the Others are excluded from being included in and contributing to modernity (and modernisation). They are silenced and often relegated to the traditional and pre-modern. The Others are also thought to be inferior to the modern, too. As Martinelli succinctly puts it, singular modernity is "a model in a double sense: on the one hand, it was a theoretical simplification of empirical reality [...]; on the other, it portrayed a desired course of action and provided a criterion for judging individual and collective behaviour actors and government policies" (Martinelli 2005, p. 60; my emphasis). In short, theorising modernity in singular terms is oppressive in at least two senses. First, it fails to give proper recognition to the Others. Second, it also consolidates an unequal relationship between the West and the Others.

Together, the two broad lines of criticisms have forced researchers to rethink their approaches to modernity. What is crucially missing in the idea of singular modernity is a sufficient attention to the diversities and variations of modernity within and without the West, as well as proper acknowledgement of the local experiences and histories in constructing narratives of modernity. Hence, any approaches that attempt to overcome the problems with the idea of singular modernity must be, in one way or another, pluralistic. This shift from singular terms to plural terms has asserted the indispensable role of traditions in shaping any modern narratives, and thereby their role in our self-interpretation and selfunderstanding. The result, therefore, is the possibility of other forms of modern identity, which are based on other value systems. If this is so, then normative judgements supported by the other forms of (modern) identity can be of a different nature than those supported by the modern and late modern self discussed in Chapter Two. Accordingly, their normative judgements of digital media and digitally-mediated practices can differ significantly, too. Hence, it is important to

\footnotetext{
7 For example, see Bhambra (2007, Chapter 1). Also, see Argyrou (2005) for a more specific critique targeted at Giddens's and Beck's reflexive modernity (or reflexive modernisation).
} 
resist the temptation to reinterpret prudential appraisals only in terms of views of the good life embodied in the modern and late modern self.

\subsubsection{Towards Plural Modernities}

To overcome the deficits of singular modernity, researchers have sought to account for the plurality of modernities in various ways. Yet, whichever way they proceed, they have to face two serious questions which follow from the plurality of modernities. First, what is modernity if (i) there is no fixed set of characteristics, e.g. '-isms' and/or '-isations', inherent to it and (ii) there is no sharp distinction between 'traditional' and 'modern'? Second, how do different modernities differ (or, in what sense we can say one modernity is different from another)? These questions are particularly important for the present study because we have to know what is modern in order to meaningfully discuss in what sense Confucianism is, or can be, modern. Similarly, we have to be able to pinpoint the differences between different modernities if we are to learn anything from them.

Wagner argues that the idea of singular modernity is primarily a product of conceptualising modernity as an era and as a set of institutions, because it has already pre-defined what modernity is from the very beginning (Wagner 2009, p. 250). In order to go beyond singular modernity, therefore, he suggests that we should look elsewhere than the set of processes that give rise to modernity and/or the institutional structures. Following Castoriadis, Wagner proposes that modernity should be conceptualised as a condition in which 'autonomy' and 'mastery' form the core of "imaginary significations" of modernity (Wagner 2009, p. 252). ${ }^{8}$ Autonomy and mastery, as Wagner explains, refers to "the idea of [...] human being as the knowing and acting subject, on the one hand, and on the other, the idea of rationality of the world, i.e. its principled intelligibility" (Wagner 2009, p. 252). In short, modernity refers to a condition in which the meaningfulness of subjects is not given but is self-defined. Conceptualising modernity as a condition (or, more precisely, as both interpretations of and responses to the condition) provides room for the plurality of modernities in that no fixed interpretation or response is required. There can be different interpretations and responses, which are equally legitimate for understanding and responding to the condition of modernity. In this

\footnotetext{
8 As Wagner explains, "by [imaginary signification], Castoriadis refers to what more conventionally would be called a generally held belief or an 'interpretative pattern"' (Wagner 2009, p. 252).
} 
way, modernity can also be seen as "an open-ended horizon in which there are spaces for multiple interpretations" (Kaya 2004, p. 37) or an "open-ended human condition, in which human beings have the opportunity to develop their own lifeperspectives" (Kaya 2004, p. 39).

Yet, conceptualising modernity as a condition by itself cannot tell us what the different interpretations and responses are and how they come about. Hence, Wagner proposes to complement the conceptualisation of modernity as a condition with the conceptualisation of modernity as an experience as well (Wagner 2009, p. 253). It is people's experience of modernity that brings about specific interpretations of and responses to it. Therefore, by looking into people's experience in light of modernity, it allows us to say what the different interpretations and responses really are, and it also provides us the explanations of why they are interpreted and responded in some, but not in the other, ways. Hence, conceptualising modernity as both a condition and an experience offers an answer to the question of 'what is modernity?', and, at the same time, it leaves open the specific characteristics that modernity exhibits.

If modernity can be conceptualised as interpretations of and responses to the condition of modernity and people's experience underlying them, then it is possible to distinguish various modernities by referring to their roots. By 'roots', I mean the point of reference for the interpretations, responses and experience. Here, traditions appear to be a proper candidate for the roots. This is exactly what Andrew Feenberg, a philosopher of technology, has done in theorising Japanese alternative modernity. Through an analysis of Nishida Kitarō's responses to Western cultures and philosophy, Feenberg has shown that an alternative understanding of modernity is a real possibility (Feenberg 1995). ${ }^{9}$

9 I agree with Feenberg and other proponents of alternative modernities that various modernities should be understood by tracing back to their own traditions. But, I disagree with them in that an alternative modernity, as they have conceptualised, risks being reduced merely to a reaction to Western cultures, i.e. it is the interpretations of and responses to an original and/or a dominant modernity of the West. In other words, an alternative modernity is conceptualised as nothing more than a condition of encounter.

However, I think such a conceptualisation of modernity is unnecessarily limited. For instance, it seems to suggest that the interpretations and responses are only about one expression of the condition of modernity, namely the West's. Without any doubt, the encounter of the West's interpretations of and responses to the condition of modernity is a catalyst to many non-Western societies. Yet, by equating them with the condition of modernity, proponents of alternative modernities have overlooked the fact that neither 
Accordingly, the differences between various modernities have to be understood in social, cultural and historical terms. By looking at local experiences and histories in which the condition of modernity is being interpreted and responded to, we can see how different modernities come into life. Still, it is sensible to question in what sense we can say that one modernity is really different from another. For example, Volker H. Schmidt has charged the proponents of plural modernities to have multiplied modernity unwarrantedly by asking "[f]or instance, is Japan significantly more different from Spain than Denmark or Britain or Greece are? And does contemporary Japan have more in common with premodern Japan than with, say, contemporary Canada or Germany?" (Schmidt 2006, p. 81)

The question posed by Schmidt is indeed a serious one for the proponents of plural modernities. However, I take this question to be an empirical one rather than a theoretical one. Hence, it is not to be resolved by theories alone. ${ }^{10}$ The idea of plural modernities, I think, retains both the advantages of highlighting the possibility of plural interpretations of and responses to the condition of modernity and the consciousness of not to assimilate local experiences and histories in a purported universal and, very often, Western model.

In this brief detour to modernity, I have attempted to illustrate a way to discuss plural modernities. This way of discussing plural modernities should make room for different forms of modern identity as well, that is-different ways of selfinterpretation and self-understanding via local experiences and histories. In the face of the condition of modernity constituted by the modern imaginary significations of autonomy and of mastery, people draw either explicitly or implicitly from their traditions and value systems to interpret and respond to it. In other words, the idea of plural modernities I have illustrated implies a plurality of self-interpretation and self-understanding based on various traditions and value systems. My discussion of another form of modernity identity, i.e. Confucian modern identity, therefore, has

autonomy nor mastery is an invention of the West. It is only specific ways of understanding autonomy and mastery, e.g. in the visions of the Enlightenment and Romanticism, which are distinctively theirs. In this respect, I think it is more appropriate to speak of multiple modernities or plural modernities.

10 Schmidt (2006) too admits that it is not merely a theoretical question, but also an empirical one. 
to start with the Confucian tradition and its value system, which form the basis of interpretations of and responses to the condition of modernity.

\subsection{Confucian Dao, Harmony and Personhood}

It is commonly agreed among researchers that contemporary Chinese culture-and, in general, East Asian cultures-is distinct from European and North American cultures in many important aspects. For instance, it has been noted that contemporary Chinese culture is predominantly shaped by Confucianism, whereas the (late) modern European and North American cultures originate from the Enlightenment and Romanticism. Researchers have also pointed out that Confucian thought and the visions of the Enlightenment and Romanticism have engendered distinct modes of self-interpretation and self-understanding. In the remainder of this chapter, by looking at the Confucian tradition, I substantiate the view that the Confucian self, even in its contemporary manifestation, is indeed unlike the modern and late modern self I have so far discussed. This, in turn, implies that the Confucian view of the good life, which is embodied in the Confucian self, is different from the views of the good life discussed in Chapter Two.

Immediately, there are two major challenges for this task. ${ }^{11}$ First, the Confucian tradition covers an enormous field of study including its metaphysics, epistemology and ethics, which is impractical, if not impossible, to include in one section. Second, there are numerous, conflicting interpretations of Confucianism from its early history to the present. Hence, it is perhaps more appropriate to speak of many Confucianism $s$ than the Confucian tradition. To answer the first challenge, I restrict my discussion only to those notions that are most relevant to the Confucian mode of self-interpretation and self-understanding and the Confucian view of the good life, i.e. Dao (道), harmony ( $\mathrm{He}$ 和) and personhood. To answer

\footnotetext{
11 Although it is commonly acknowledged that (contemporary) Chinese culture is predominantly Confucian, this claim is nothing but controversial without qualifications. Hence, there is a third challenge requires answering, namely to what extent (contemporary) Chinese culture is really Confucian? However, to answer this third challenge will lead us too far away from the current discussion. It is clear that Chinese culture is shaped by other traditions as well, e.g. Daoism, Chinese Buddhism (i.e. Ch'an (襌) Buddhism), Chinese Marxism, etc. Following other researchers in the field, I maintain that Confucianism remains the bedrock of Chinese culture, and that other traditions contribute to Chinese culture insofar as they interact with and being assimilated by Confucianism. In short, Confucianism has remained as the most dominant value system throughout Chinese history. See, e.g. Tu (1993), HWANG (2001).
} 
the second challenge, I try to elaborate a least controversial interpretation of Confucianism by identifying the basics that are shared or, at least, can be shared by various interpretations. ${ }^{12}$

\subsubsection{Dao: Foundation of Confucianism}

The term 'Dao' is often linked to Daoism, i.e. a major rival to Confucianism, but, as a matter of fact, it is one of the most important notions in Chinese thought. At the same time, Dao is also one of the most elusive notions too. It is so because the notion has been the focus of debates within and between various Chinese philosophical traditions, resulting in a variety of understandings of the term. ${ }^{13}$ Yet, for its importance in Chinese thought, an account of Dao is necessary in order to have a proper understanding of it. Since this chapter focuses on Confucianism, I shall look specifically at the notion of Dao in Confucianism. Confucians believe that the universe is organised and governed by a specific principle, which they called Dao. While Confucians use the term 'Dao' to refer to the organising and governing principle of the universe, the term is also being used in other ways. In a summary of the meaning(s) of the term 'Dao', van Norden points out that

"[Dao] has several related senses. (I) The original sense was "way," in the sense of "path" or "road." It came to mean (2) "way," in the sense of "the right way to do something," or "the order that comes from doing things in the right way," (3) a linguistic account of a way to do something, or "to give a linguistic account," (4) a metaphysical entity responsible for the way things act." (van Norden 2000, p. 24)

12 Many have already pointed out that Chinese philosophy differs from both the AngloAmerican analytic tradition(s) and/or the European continental tradition(s) in its methodologies, fundamental assumptions and core concepts. These differences present a real challenge to do comparative philosophy. In a paper, LIU JeeLoo (2009) has summarised various approaches that aim to overcome this challenge. In this chapter, I adopt what she labels the analytic approach to Chinese philosophy. Such an approach is characterised by its focus "on the conceptual analysis of philosophical ideas, the clear formulation of argumentation, the investigation of philosophical problems and their solutions, and the posing of hypothetical thought experiments to test one's intuition. [It usually] begins with the original text, but goes further to construct a philosophical system for the original Chinese philosopher who did not do so in his writing" (J. LIU 2009, pp. 8-9; also, see D. Wong 2009).

13 This point is aptly captured by the title of A.C. Graham's (1989) book, i.e. Disputers of the Tao [Dao]: Philosophical Argument in Ancient China. 
As the summary shows, Dao has different connotations, i.e. it is, at the same time, metaphysical, epistemological and ethico-political. In its metaphysical connotation, i.e. (4), Dao is most often associated with Heaven (Tian 天). In Confucian thought, Heaven refers to the universe, and/or when in conjunction with Earth ( $D i$ 地) to the nature and the material world. Confucians believe Heaven is the source of all meaning and values. Heaven is said to have its own dao, i.e. the Dao of Heaven or the Heavenly Dao (Tiandao 天道), which is the principle that organises and governs the universe and/or the material world. Although the exact meaning of Heaven is disputed in Confucianism, there are two common understandings of it. In the spiritual, religious understanding of Heaven, it is understood as the Supreme Being, who is responsible to organise and govern the material and human world (Ivanhoe 2007, HUANG 2007). And, in the naturalistic understanding of Heaven, it is conceptualised as the nature akin to the Natural Law tradition in modern European philosophy (J. LIU 2007). Either way, Heaven is conceived as the ultimate source of normativity.

It should be pointed out that the normative role of Heaven in Confucian thought is not merely negative but also positive and proactive, and that the worldview powered by Heaven is not deterministic. For Confucians, Heaven does not only sanction and rectify wrongdoings; it also nourishes things. For human beings, Heaven bestows them with the potential to become attuned to Heaven. Everything has its own dao, which is an instantiation of the Heavenly Dao. These daos specify how things ought to be (or ought to be done). Somewhat paradoxically, however, while the Dao of Heaven specifies the telos and ideal of human beings, and in spite of the significance of Destiny (Ming 命) in Confucian thought, it does not predetermine people's course of action. Most notably, as it is stated in The Analects 15.29 (Lunyu 論語), "[h]uman beings can broaden the Way-it is not the Way that broadens human beings" (Slingerland 2003, p. 185). It is so because only through human beings can the meaning and values embodied in Heaven can be realised. In other words, the Heavenly Dao does not dictate human and social affairs; and, human beings, as followers of Dao, remain firmly at the centre of the universe.

Following from the discussion above, the epistemological connotation of Dao should be clear. Since Dao (of Heaven) refers to the principle that organises and governs the universe and/or the material world, it is epistemologically significant insofar as it specifies the good and the right ways to do things, i.e. (2). I will not discuss the details of the epistemological connotation of Dao here, as it is not directly relevant to the present study. Still, it is worth noting that there is no sharp distinction between theoretical and practical knowledge in early Chinese thought; 
or, that the importance of theoretical knowledge is often downplayed. Chad Hansen has supported this view from a philosophical-linguistic perspective. He points out that the view of language in early China is not truth-apt but action-guiding. Language uses are evaluated in terms of right-wrong (shi-fei 是非) not truth-false. This non-representational view of language has disfavoured the pursuit of theoretical knowledge, which aims primarily at the Truth (Hansen 1992, pp. 14-25, 51-52 \& 85-86). Furthermore, as Hansen and other commentators point out, the primary concerns of Confucians - and, in general, (ancient) Chinese thinkers-are practical in nature (see, e.g. HUANG 2005, pp. 514-517; D. Wong 2009). As a result, the epistemology of Confucianism also differs notably from other philosophical traditions.

Finally, the ethico-political connotation of Dao should also be clear, too. As previously noted, Heaven is the ultimate source of normativity, which includes epistemic normativity as well as moral and political normativity. Since Confucians believe that every dao is an instantiation of the Heavenly Dao, the organising and governing principles for human and social affairs, i.e. the Dao of Humanity or the Human Dao (Rendao 人道), is thus also an instantiation of the Heavenly Dao. The Human Dao refers to the way in which human beings should live. ${ }^{14}$ Here, it is important to point out the uniqueness of the Human Dao as an answer to the question of 'how should people live?'. The Human Dao mainly focuses on people's acquisition of virtues ( $D e$ 德) but not on establishing universalisable moral norms and principles, as Confucians believe virtuous people already know how to live. This focus on the acquisition of virtues can be explained, in part, by the relationship between human beings and Heaven in Confucian thought. While the Human Dao gets its authority from the Heavenly Dao, it is a mistake to see them as two separate principles. In Confucian thought, Heaven and Humanity are characterised by the Oneness of them, as the opening passage of the Confucian classic The Doctrine of Mean (Zhongyong 中庸) stated:

"What Heaven (Tian, Nature) imparts to man is called human nature. To follow our nature is called the Way (Dao). Cultivating the Way is called education. The Way cannot be separated from us for a moment. What

14 The Human Dao also has a political dimension, i.e. it is the way the ruler should rule the people. Although a separation of ethics and politics in Confucianism can only be done artificially, I shall maintain this separation and ignore the political dimension of Confucianism unless it is necessary to take it into account. 
can be separated from us is not the Way..." The Doctrine of Mean, I (Chan 1954, p. 94)

People can cultivate their nature to achieve their ideals because Heaven has bestowed them with the potential to do so. People should cultivate their nature because it is required to realise Dao. The emphasis, therefore, is on self-cultivation. The Oneness of the Heavenly Dao and the Human Dao is too illustrated by Confucius's (Kongzi 孔子) method of learning the Dao, i.e. "[to] study what is below in order to comprehend what is above" (The Analects 14.35; Slingerland 2003, p. 168). Confucius's method brings forth two points concerning Dao. First, it affirms the Oneness of the Heavenly Dao and the Human Dao, i.e. the former is known through the latter. Second, it also affirms the priority of practicality in Confucianism. As Dao manifests itself in human and social affairs, Confucians believe that it is only pertinent to investigate worldly affairs, i.e. the Human Dao, rather than the unworldly, abstract Dao of Heaven. ${ }^{15}$

To sum up, Dao is the metaphysical, epistemological and ethico-political foundation of Confucianism. While, for some, the metaphysics and epistemology associated with Confucian Dao have become obsolete and doubtful because of their alleged incompatibility with a scientific worldview, the ethico-political dimension of Dao remains significant for the majority of contemporary Confucians. Hence, Dao is significant for the current discussion as it underlies the (contemporary) Confucian view of the good life. Based on Dao, as I shall illustrate, a Confucian account of ethics, which is unlike typical Western ethical theories, offers an alternative way to look at the good life.

\subsubsection{Harmony in Confucianism}

The emphasis on the Oneness of Heaven and Humanity (Tianren Heyi 天人合一) in Confucian thought has exemplified the importance of harmony in Confucianism. Confucians believe the ideal relationship between human beings and Heaven is not confrontational but harmonious, i.e. human beings are not against Heaven, neither is Heaven opposed to human beings. Depending on the view of Heaven, various

\footnotetext{
15 Similar claims concerning the relationship between (the Dao of) human beings and (the Dao of) Heaven can be found in other Confucian classics, e.g. Mencius 7A1 (Mengzi 孟 子):

"To fully apply one's heart is to understand one's nature. If one understands one's nature, then one understands Heaven. To preserve one's mind and nourish one's nature is the means to serve Heaven." (van Norden 2001, p. 149)
} 
interpretations of the harmonious relationship between human beings and Heaven can be elaborated. For example, it is argued that human beings and Heaven are of the same nature ontologically. Or, in the spiritual, religious understanding of Heaven, the Oneness between human beings and Heaven is to be achieved by following the will of Heaven. ${ }^{16}$ Yet, as an ideal relationship, harmony does not only hold between human beings and Heaven; it is an ideal relationship for within an individual and between individuals at the level of family, society, and the world. In Confucianism, special attention is given to interpersonal harmony, as Confucians believe not only that it exerts enormous influence on intrapersonal harmony, but also that it is a prerequisite for intrapersonal harmony. ${ }^{17}$

As LI Chengyang points out, in Confucian thought, the harmony-disharmony distinction plays a role similar to distinctions of right-wrong, of good-bad, and of success-failure (LI 2006, p. 588). In this respect, harmony can be conceived as a normative standard of Confucianism. ${ }^{18}$ Its role in defining the view of the good life, therefore, should not be overlooked. In order to see what harmony demands, however, it is necessary first to explicate the meaning of the notion. In an analysis of the notion, YU Kam-por has succinctly summarised four key features of harmony in Confucian thought. According to YU,

\section{Harmony is not complete agreement.}

16 For an overview of various interpretations of the Oneness between Heaven and Humanity, see K. WANG (2007).

17 While Confucians tend focus on interpersonal harmony, there are discussions of intrapersonal harmony in Confucianism as well. This is evidenced by the Confucian dictum in The Great Learning (Daixue 大學), i.e. "to cultivate the person", "to regulate the family", "to order well the States", "to illustrate illustrious virtue throughout the empire" (Legge 1861, pp. 221-222). And, it is stated in The Great Learning VII.1 that,

"[...] 'The cultivation of the person [...]' may be thus illustrated: - If a man be under the influence of passion he will be incorrect in his conduct. He will be the same, if he is under the influence of terror, or under the influence of fond regard, or under that of sorrow and distress." (Legge 1861, p. 232)

In the original text, the literal meaning of "to cultivate the person" is to cultivate the body

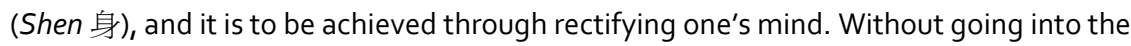
details, it is clear from the passage that the interpersonal relationships, e.g. in family, States, and the empire, are too depended on intrapersonal state, i.e. person (or body). See, R. Wang (2010) for a discussion of the importance of (harmonious) body in Confucian ethics. Moreover, the priority of interpersonal harmony in Confucian thought can be explained partly by their view of personhood. See, section 4.2.3.

18 In effect, LI has gone further to argue that harmony is the normative standard of Confucianism. See, LI (2006, p. 589). 
2. Harmony is not unprincipled compromise.

3. Harmony is balancing one thing with another one.

4. Harmony is the mutual complementation of acceptance and rejection. (K.P. Yu 20I0, pp. 2I-25)

To clarify the notion of harmony in Confucianism, each of the four features requires further explanation. The first and second features remind us of the need to distinguish sameness (Tong 同) from harmony. Harmony and sameness are explicitly set apart in Confucianism. For instance, in The Analects 13.23, it is stated that: "The gentleman [i.e. Junzi] harmonises $(\mathrm{He})$, and does not merely agree (Tong). The petty person agrees, but he does not harmonise" (Slingerland 2003, p. 149). At the heart of the difference is the idea of creative dynamics, which Confucians believe to be essential to human flourishing. Aiming only at complete agreement leads to mutual reinforcement, but it does not promote creative exchanges. In other words, it only maintains the status quo, and it does not contribute to advancement or growth. Harmony differs from complete agreement because it is mutually beneficial to the involved parties, which is made possible by their creative dynamics. Hence, unlike sameness, which precludes differences and diversity, harmony presupposes them.

Similarly, harmony is not unprincipled compromise, as compromise per se is not conducive to mutual enrichment. For Confucians, unprincipled compromise is even detrimental to harmony. ${ }^{19}$ Since Confucians believe that people and things have their roles and functions, unprincipled compromise is, in effect, a form of suppression and/or repression of their proper roles and functions, which is going to result in disharmony. Hence, a relationship sustained by an unprincipled compromise cannot be considered to be genuinely harmonious. In other words, a genuine harmonious relationship must be backed by reasons.

The third and fourth features explain how harmony is to be achieved. As YU notes, three types of analogy are often used in the Confucian classics to explain the notion of harmony, i.e. the cooking analogy, the music analogy and the health analogy (K.P. YU 2010, pp. 18-20). What is in common in those analogies is that their successes depend on coordination between the elements involved, where the

\footnotetext{
19 As Li (2006) rightly notes, the notion of harmony (or harmonisation) presupposes differences. So, merely aiming at eliminating differences, unprincipled compromise is, in effect, opposed to harmony.
} 
coordination calls for the elements (i) to perform their own roles and functions, (ii) to relate to other elements in an appropriate way and (iii) to not to over-power, or even dominate, other elements. Accordingly, harmony requires balancing things so that they complement and support each other. Those analogies also recapitulate the point that harmony, unlike sameness, is conducive to mutual enrichment because the outcome is always larger than the sum of its parts.

The notion of mutual complementation of acceptance and rejection may appear to be puzzling at first. It refers to the idea that harmony "is achieved only if we are able to appropriate what is acceptable in what is objectionable and denounce what is objectionable in what is acceptable" (K.P. YU 2010, p. 23). This notion stems from the recognition and acceptance of the complexity in real life and from the doubt of an absolute, decontextualised goodness or rightness. For Confucians, what is good or bad and what is right or wrong can only be determined in a concrete situation, in which particularities become salient. Harmony is achieved by taking into account various possibilities in that situation. In other words, harmony involves contextualised and holistic thinking. In practice, as LI (2006) points out, concrete situations are seldom fixed; therefore, harmony can only be ensued by continuous negotiation and adjustment. In this respect, it is more appropriate to conceptualise harmony as a process, i.e. harmonisation, than a relational property or a state of affairs.

\subsubsection{Confucian Person}

A study of Confucianism, for the purpose of illuminating its account of ethics and its view of the good life, will be incomplete without considering the Confucian notion of personhood. In the modern, Western view of personhood, which is preoccupied by a search for basic standards for the ascription of personhood, whether or not a being is a person is often determined solely by the possession of certain characteristics of individuals. This view tends to define 'person' in terms of inner characteristics; and, a person is typically conceived of as an independent, rational and self-determining being. ${ }^{20}$ Following Michael Walzer (1994, Chapter 1), we can call this view of personhood a thin notion of personhood because it is a minimalist definition of 'person', which is devoid of social, cultural and historical links and meanings. In contrast to this view, we can call the Confucian notion of personhood a thick notion of personhood because, as I shall illustrate, it goes 
beyond a minimalist definition and is inherently social, cultural and historical. At risk of oversimplification, from the Confucian perspective, the notion of personhood provides answers to ethico-political issues at every level. It is so because the Confucian notion of personhood is ethical by definition. As Erika Yu and FAN Ruiping have nicely characterised, the Confucian person is relational, developmental and virtue-based (Yu \& FAN 2007, pp. 175-176). In order to see how the Confucian notion of personhood answers the ethico-political issues, different characteristics of the Confucian personhood, i.e. relational, developmental and virtue-based, must be elaborated in more detail. ${ }^{21}$

Few commentators will dispute the claim that the Confucian person is relational (see, e.g. D. Wong 2004a; Lai 2006, pp. 30-34; J. YU 2005, 2008; Bockover 2010). Confucians think that human beings are inherently social and interdependent. David Wong points out that Confucianism presupposes a "social conception of the persons", which refers to the view that human beings are "biological organisms and become persons by entering into relationship with others of our kind." He also points out that Confucians posit human beings to be interdependent by nature, as human beings "need the help of others to develop as agents" (D. Wong 2004a, pp. 420-421). ${ }^{22}$ In other words, Confucians think that human beings are inescapably born into a web of social relationships, and that they can only mature within the web of social relationships. Most importantly, however, is that relationality is constitutive of the Confucian self in the sense that (morally) right decisions and actions are seen as co-authored achievements (D. Wong 2004a, pp. 424-427). This is exactly why harmonisation, which involves continuous negotiation and adjustment of interpersonal relationship for the sake of mutual

\footnotetext{
21 A serious misconception of the Confucian self, which is often associated with the Confucian notion of personhood, has to be cleared up first. It is often claimed that Confucianism prioritises the community (or collective) over the self (or individuals). This claim amounts to a distortion of Confucianism, i.e. to say that Confucians prioritise the community (or collective) requires a sharp distinction between the community (or collective) and the self (or individuals), but, as many commentators have already pointed out, there is no sharp distinction as such in Confucianism. See, e.g. D. Wong (2004a) and Lai (2006, pp. 30-34).

22 David Wong has labelled the interdependent nature of personhood "the developmental sense of relationality" (D. Wong 2004a, p. 421). His use of the term "developmental" differs quite significantly from the use of "developmental" in Yu and FAN (2007), where Yu and FAN use the term to characterise the Confucian notion of personhood as a process. In this section, I am using the term in the sense suggested by $\mathrm{Yu}$ and FAN.
} 
enrichment, is seen as an ideal in Confucianism. Of course, there are similar relational views of personhood in other philosophical traditions as well. What distinguishes the Confucian notion of personhood from them is the weight it places on familial relationships and on social role.

Confucians take familial relationships to be of the utmost importance. They argue that family is the very first social context where human beings learn to relate to and interact with others appropriately. They also argue that natural familial affections found in the parent-child relation form the basis of 'love' towards others. For Confucians, therefore, family plays a principal role in shaping one's personhood. Moreover, the importance of familial relationships in Confucianism is further illustrated by their effort to model all socio-political relationships upon familial relationships.

Also important to the Confucian notion of person is the social roles one occupies. In Confucianism, to be a person requires one to stand in some relations to others appropriately; and, to stand in some relations to others appropriately means that he assumes and fulfils the responsibility the relations require. Consider, for example, the five basic types of human relations (Wulun 五倫) in Confucianism: parent-child, sibling, husband-wife, ruler-minister and friendship. According to Confucians, each pair of human relations embodies a set of proper conducts and attitudes, e.g. whether one is a parent or not is determined by his acceptance and fulfilment of the responsibility to his child through following the prescribed set of proper conducts and attitudes; and, the same holds for other human relations too. In short, to be a Confucian person is to stand in some relations to others, and the relations are to be understood prescriptively. So, a Confucian person cannot be defined independent of others; he has to be defined in terms of the social roles a person occupies. ${ }^{23}$

To call the Confucian person developmental is to see the Confucian personhood as an on-going process. Confucians regard personhood to be neither static nor given. It is not static because persons cannot be identified with any sets of characteristics of individuals. It is not given because, in Confucian thought, while every human being is endowed with the potential to be a person, whether or not human beings become persons depends on their preservation and cultivation of the potential bestowed on them. In other words, human beings are not born persons,

\footnotetext{
23 Accordingly, Confucian ethics can be understood as a form of role-based ethics. See, Nuyen (2007, 2009).
} 
they learn and practise to be persons. In this respect, the Confucian notion of personhood is aptly labelled by David Hall and Roger Ames (1987) as "personmaking". Since human beings are endowed with the potential to be persons, they only need to preserve and cultivate the potential in order to become persons. Hence, "person-making" is essentially about self-cultivation. In Confucianism, given the relational nature of personhood, self-cultivation is about learning and practice of relating to and interacting with others appropriately. While one can learn and practise to be a person, one can also degrade into a non-person due to failure to assume and fulfil the required responsibility. For example, a parent who fails to provide sufficient love, care and guidance to his child is merely a beast in the Confucian perspective. Since there is the possibility for a person to degrade into non-person, being a person is literally an on-going process. In effect, the paradigmatic person (Junzi 君子) in Confucianism is a person who is always able to respond to different relationships and in various concrete situations with propriety.

Finally, it should be obvious why the Confucian notion of personhood is virtue-based. Confucians think that personhood is defined in terms of virtue(s). For instance, in The Doctrine of Mean 20, it is stated that: "Humanity (Ren) is [the distinguishing characteristic of] man" (CHAN 1954, p. 104). Ren, often translated as 'humanity', 'benevolence', or 'goodness', is seen as the ultimate virtue in Confucianism (D. Wong 2008). Here, it is important not to mistake the quote to claim that having the virtue in itself suffices for personhood. Recall the developmental nature of the Confucian person: to be a person, it is not enough to have the virtue, what is necessary is the realisation of the virtue within a web of social relationships. Since Confucians think that the realisation of ren requires one to live a specific way of life prescribed by the social roles one occupies, the Confucian notion of personhood has to be seen as a thick notion.

\subsection{Confucian Ethics: The Basics}

To sum up the discussion so far, I have elaborated three central notions in Confucianism, i.e. Dao, harmony and personhood. In contrast to typical Western ethical theories, those notions proffer an alternative account of ethics and a different view of the good life in Confucianism. In this section, using the notions I have so far elaborated, I outline an account of Confucian ethics, which, I hope, can 
form the basis for the subsequent discussion. ${ }^{24}$ Before outlining the account of Confucian ethics, however, it is helpful to begin by looking at the relation between the right and the good in Confucianism.

Typical Western ethical theories assume a separation of the right and the good, as well as a prioritisation of either one over the other. ${ }^{25}$ Unlike those theories, neither the distinction nor the prioritisation of the right and the good is important in Confucian ethics. ${ }^{26}$ I have already illustrated that Dao is the ultimate source of normativity in Confucianism; it is constitutive of both the right and the good. For instance, as the goal and ideal of human beings in Confucianism is the Oneness of Heaven and Humanity, which is to be attained through the realisation of Dao, the realisation of Dao has epitomised human flourishing, i.e. the good. At the same time, Dao has also delimited the right ways of its realisation, too. As Erin Cline illustrates it with the following passage from The Analects 4.5: "[w]ealth and honors are things that all people desire, but unless they are acquired by following the Way $[D a o]$ they are not worth having. Poverty and disgrace are things that all people hate, and yet unless they are avoided by following the Way [Dao] they are not worth avoiding" (Cline 2009, p. 113). What this passage conveys is that even if something is good, when pursuing it in a way that goes against Dao, a person should not do so. Here, note that the normative standard is not the right per se but Dao. Referring to this and other passages in The Analects, Cline argues that Dao is prior to both the right and the good in Confucian ethics. Whether her claim that Dao is prior to the right and the good is true or not, I think, she has rightly emphasised that the right and the good are intermingled in Confucian ethics.

\footnotetext{
${ }^{24}$ In this section, I only focus on Confucian normative ethics. In a paper, LIU JeeLoo (2007) has offered an interesting analysis of Confucian meta-ethics in terms of moral realism. While her analysis is not uncontroversial, I think she is right in conceptualising Confucian ethics as a type of moral realism. Yet, because the details of Confucian metaethics will have little import to the current discussion, I shall leave aside the questions concerning Confucian meta-ethics.

25 The separation of the right and the good in typical Western ethical theories is, I think, best captured by Charles Taylor's claim that "moral philosophy [...] has tended to focus on what is right to do rather than on what is good to be, on defining the content of obligation rather than the nature of the good life" (Taylor 1989, p. 3).

26 Cf. Cline (2009). Certainly, some have argued the good is prior to the right in Confucianism. But, as it should be clear later, a prioritisation of the good can only be done artificially. I believe the prioritisation of the good is proposed mainly for heuristic purpose, namely to contrast Confucianism with (Rawlsian) liberalism, which generally holds that the right is prior to the good.
} 
The claim that the distinction and the prioritisation are unimportant in Confucian ethics can be illustrated by the notion of harmony too. I have pointed out that Confucians regard continuous negotiation and adjustment in terms of harmony to be the right thing to do. At the same time, they also regard harmonious relationships - be they interpersonal or intrapersonal-to be essential to human flourishing. Harmony, in other words, is also good. Moreover, in the processes of harmonisation (i.e. continuous negotiation and adjustment for harmonious relations), people have to balance their self-interest, i.e. the good, with other's interest, i.e. the right; and, in this process of balancing, neither self-interest nor other's interest can take absolute priority.

Let us now turn to Confucian ethics in more detail. Given the importance of selfcultivation and virtues in Confucian ethics, many believe that Confucian ethics is best understood as a form of virtue ethics. ${ }^{27}$ Yet, in what sense can one characterise Confucian ethics as a form of virtue ethics? Justin Tiwald identifies two definitions of contemporary virtue ethics of which Confucian ethics may fittingly be labelled as virtue ethics. He notes, on one hand, that in one definition of virtue ethics, "[i]t presupposes that virtue (or perhaps approximate notions like flourishing) is more basic than rules of action and the maximisation of good states of affairs, [...] the explanatory primacy of virtue [is] definitive of virtue ethics" (Tiwald 2010, p. 56). On the other hand, he notes that virtue ethics can be defined as the view that "in engaging in higher-order moral reflection, the first or primary task should be to understand things like the nature, psychological structure and ways of cultivating good character, rather than the principles of right action or their relationship to various goods. [E]thics, when done rightly, attends to character first (Tiwald 2010, p. 60). Tiwald's characterisation of Confucian ethics with these two definitions is illuminating, and he has supported his claim by numerous recent works. Yet, as Karyn Lai notes cautiously, renditions of Confucian ethics entirely as contemporary virtue ethics might risk losing insight of Confucian ethics in translation (Lai 2006, pp. 114-116). And, the problem is especially acute in Tiwald's first definition. Confucianism, as I have elaborated, has placed special attention to social role(s) and

\footnotetext{
27 For a state-of-the-art review of Confucian ethics and virtue ethics, see Tiwald (2010). See also, Lai (2006, pp. 114-116)
} 
the need of learning and practice, which "the explanatory primacy of virtue" fails to capture in its entirety. ${ }^{28}$

In Confucian ethics, a person's social roles are morally significant because they specify his responsibility in a web of relationships. Corresponding to each of the social roles, there is a set of proper conducts and attitudes. It is only through following that set of proper conducts and attitudes, can he realise Dao, i.e. that his choices and behaviours are considered to be right and good. In this respect, social roles are both motivational and normative. They determine what we ought to do and provide us reasons for doing it. Given the importance of social roles and the corresponding sets of proper conducts and attitudes, people's learning of them is essential to Confucians. Here, to know what a person ought to do is to know what social roles he occupies and, relatedly, the sets of proper conducts and attitudes prescribed by the social roles. Yet, it has to be pointed out that learning about a person's social roles necessarily involves practice. The connection between learning and practice is exemplified by Confucian notion of rectifying names (Zhengming 正 名), i.e. to rectify oneself to fit the name. For example, a parent ought to act what a parent is supposed to act, and any failures and deficiencies on his part must be rectified to fit what the name calls for; otherwise, he is not a parent. Hence, learning always involves practice, because by learning about the social roles and the sets of proper conducts and attitudes, one is required to act out what he has learnt.

There is another route to demonstrate the importance of practice for Confucians. In the discussion of the Confucian notion of personhood, I have noted that being a person is an on-going process, in which a person needs to guard himself from degrading into non-person. Being a person, in effect, is practising personhood. Yet, the purpose of practising personhood is not only negative but positive too. Practising personhood, i.e. appropriately relating to and interacting with others in various concrete situations, enables a person to cultivate his moral sensitivity to others and to the morally significant factors in the concrete situation, which then

28 Lai has identified seven features in which Confucian ethics departs from contemporary virtue ethics, they are respectively, "[i] attention to roles and their corresponding obligations, [ii] consideration of what is right, [iii] connection between character and moral motivation, [iv] an ethic that is both act- and agent-centred, [v] the primacy of practice, [vi] a method of argumentation that prefers arbitration to adjudication and [vii] reasoned judgment based on what is the right or fitting thing to do (Lai 2006, p. 116). I think the most important among them are the (i), (v) and (vii). 
allows him to comprehend relationships and situations more accurately and, thus to respond with propriety more effortlessly (Lai 2006, pp. 117-118, 121-122).

The emphasis on practice is arguably the result of Confucian ethics's focus on the paradigmatic person in its discussions of ethico-political issues. According to Antonio Cua,

"In Confucian ethics, there is no straightforward application of moral and ritual rules. There are neither 'rules of relevance' nor 'rules of inference' for concrete moral performance... [The Confucian paradigmatic person (Junzi)] is a paradigmatic guide for ordinary moral agents by virtue of his ability to cope with the changing circumstance within the Confucian moral point of view." (Cua 197I, Pp. 52-53; quoted in Lai 2009, p. 81 )

There is no straightforward application of moral and ritual rules because Confucians recognise and accept the complexity of real life. Accordingly, there are rarely situations that are (nearly) identical so as to warrant a straightforward application of moral and ritual rules without the need to grant exceptions. At the same time, concrete situations are deemed too complex to be accounted for by any absolute standard(s). More important, perhaps, is the ideal of harmony in Confucianism. As I have illustrated, harmonisation requires a person to balance different factors in a concrete situation, it also requires him to take into account various pros and cons and to arrive at an 'in-between' position of the mutual complementation of acceptance and rejection. For Confucians, therefore, ethical deliberation must incorporate the particulars in concrete situations. ${ }^{29}$ In this respect, Confucian ethics can be seen as a (mild) form of moral particularism, as it rejects the plausibility of moral principles and their applications in concrete situations. As Lai points out, the

29 In The Analects, Confucius was famously recorded to have offered very different answers to the same question, see, e.g.

"Zilu inquired, 'On learning something, should one act upon it?' The Master said, 'While your father and elder brothers are still alive, how could you, on learning something, act upon it?' Then Ranyou asked the same question. The Master replied, 'On learning something, act upon it.'

Gongxi Hua said, 'When Zilu asked the question, you observed that his father and elder brothers are still alive, but when Ranyou asked the same question, you told him to act on what he learns. I am confused-could you explain this to me?' The Master replied, 'Ranyou is diffident, and so I urged him on. But Zilu has the energy of two, and so I sought to rein him in"' (Ames \& Rosemont 1998, pp. 146-147; quoted in Lai 2009, p. 79). 
emphasis on the paradigmatic person and the rejection of moral principles have led to questions about know-how, e.g. "how to deliberate with reasons?", "how does one learn to be a paradigmatic person or a 'competent moral judge'?" (Lai 2009, p. 81). In short, Confucian ethics regards ethical deliberation as a skill. And, ethical deliberation, being conceptualised as a skill, has reinforced the importance of practice in Confucian ethics.

Tiwald's second definition of Confucian ethics as virtue ethics, I contend, is less problematic. It is true to characterise Confucian ethics as an ethics that "attends to character first." More specifically, the aim of Confucian ethics can be thought of as self-transformation, e.g. to become a paradigmatic person. In Confucianism, such a self-transformation is to be achieved via self-cultivation. As Joel Kupperman (2010) points out, in contrast to typical Western ethical theories, which have excluded the private sphere from ethical deliberation, Confucian ethics does not distinguish the private sphere and the public sphere as clearly. It is so not only because Confucians find it difficult to divide the private and the public, as one's personhood is essentially co-authored by himself and the others within his web of relationships; it is also because Confucians believe that a person's conducts and attitudes in private life will be carried onto his public life. This is so because they believe that the kind of person one developed into over a period of time will manifest, albeit in a varying extent, in both his private and public life. As such, the ways a person lives-his lifestyles-matter, particularly if the person is interested in self-transformation (Kupperman 2010, pp. 17-22). This is why Confucians have stressed the importance of rites ( $L i$ 禮), which, to put it in somewhat simplified terms, are sets of proper conducts and attitudes prescribed by social roles. In following rites, a person internalises the set of proper conducts and attitudes; and thereby cultivating himself to realise Dao; and, simultaneously, he is self-transforming in the direction of the paradigmatic person. In short, the scope of Confucian ethics is broader than typical Western ethical theories: it looks at the possibility to amelioration of oneself; and, it also looks at the private sphere by emphasising lifestyles that are conducive to selftransformation.

\subsection{On the Confucian Way of Life: A Contemporary Account}

In the previous sections, I have elaborated the three major notions in Confucianism, and outlined an account of Confucian ethics. However, as I have only looked at the notions in isolation, the account is ahistorical. Insofar as my aim is to explicate the view of the good life in Confucianism in the present day, i.e. a modern Confucian 
view of the good life, an ahistorical account is insufficient. In order to properly speak about a modern Confucian view of the good life, it is necessary first to show that Confucianism is a living tradition. In other words, I have to demonstrate not only that Confucianism is compatible with modernity, it is also constitutive of its own modernity. Following Wagner, I have proposed that modernity is to be understood as interpretations of, responses to and experience in the condition of modernity. In this respect, to explicate the liveliness of Confucianism is to demonstrate its place in the interpretations, responses and experience.

However, it is not my intention to document, historically, the Chinese interpretations of, responses to and experience in modernity, as it will exceed the scope of this section. Instead, I want to show, conceptually, the influence of Confucianism in Chinese modernity through a brief historical narrative of its interpretation of the concept of the individual because of its intimate link to autonomy. Secondly, I examine the Confucian notion of rationality, i.e. zhongyong rationality (中庸理性), and describe how it provides another way to conceive mastery. In this way, I hope, I can demonstrate the lasting influence of Confucianism, the validity to speak of a Confucian modernity or a Confucian modern identity and, relatedly, a modern Confucian view of the good life.

\subsubsection{Confucianism, Individuality and Autonomy}

To reiterate, autonomy refers to the idea that human beings are knowing and acting subjects. This idea gives rise to a specific concept of the individual, i.e. persons are distinct from others-including both the nature and the society-and can know and act on their own will. However, by knowing and acting on their own will, it does not entails individuals must be (hyper-)individualistic, i.e. that they are separated from the nature and/or society. In other words, the notion of autonomy itself allows room for multiple interpretations. At the same time, as the concept of the individual does not come into existence ex nihilo, the social, cultural and historical complexities within a society also shape and limit the potential meanings of autonomy. In the West, the notion of autonomy is often interpreted (hyper-)individualistically as in the disengaged self, expressive self as well as the later reflexive self, accompanying with this interpretation of autonomy is a concept of the individual, who is separated from, and even against, others and the state. Given the intimate relation between autonomy and the concept of the individual, the concept of the individual can serve as a proxy for our examination of autonomy. By looking at the influence Confucianism has on the emergence and change of the concept of the individual, I hope to demonstrate its place in a Confucian modernity. 
Researchers have already noted that the Chinese concept of the individual diverges from the (hyper-)individualistic concept of the individual usually prevails in the West; they have also pointed out that those differences can, by and large, be attributed to the influence of Confucianism in the concept's emergence and subsequent development (see, e.g. GU 2001; X.-H. LIU 2004, pp. 37-41; JIN \& LIU 2009, pp. 151-179; XU 2009; YAN 2010). The Chinese concept of the individual first explicitly surfaced in the 19th century, when China suffered from continuous defeat and humiliation, and being forced open to the West. Chinese intellectuals at that period attempted to introduce a Western concept of the individual, together with other concepts, e.g. science and democracy, in the hope of modernising China and thereby to save the country. However, as an early slogan of "Chinese-body ( $T i$ 體; also means ground) with Western-use (Yong 用; also means manifestation)” suggests, Western concepts, including the concept of the individual, were introduced and adopted only for instrumental purpose. A concept of the individual as an acting and knowing subject was important, because it was believed that an individual's independence is the prerequisite of the country's independence (JIN \& LIU 2009, p. 159). Accordingly, individuals, though viewed as independent, were still subsumed under the country, and not against it. Moreover, because of the duality of ti-yong, Confucianism was not rejected by Chinese intellectuals; Confucianism remained in place in the private sphere; and, the concept of the individual was only applicable to the public/political sphere (JIN \& LIU 2009, pp. 161-168).

Confucianism, however, was subjected to two severe critiques in China after early failed attempts to modernise China. The first critique happened during the May Fourth Movement, i.e. an intellectual revolution and social, cultural and political movement in early 1920s, when Confucianism was discredited as the barrier to modernisation. The second critique is led by MAO Zedong, who sought to thoroughly overthrow Confucianism and replace its values with Communist values. While both critiques are explicit attacks of Confucianism, they are, paradoxically, Confucian in nature. First, as Gloria Davies (2007) has documented, the Chinese critical discourse, from its very beginning, is driven by a "crisis mentality" (youhuan yishi 憂患意識) and is directed at the country's well-being and the cultural quality of the people (Davies 2007, pp. 16-18). This mentality is deeply Confucian as it rested on the ideal of the scholar-officials in Confucianism. Hence, the attacks on Confucianism in the May Fourth Movement were stemmed from Confucianism itself. More importantly, however, as GAO Like (2008) points out, attacks on Confucianism were primarily about the institutionalised practices of Confucianism 
in both the private and public sphere; and, the Confucian values per se were not so much attacked. In a similar vein, although MAO Zedong explicitly rejected Confucian values and replaced them with Communist values, the Maoist value system and its ethic were both structurally and substantively similar to Confucianism. It is particularly true if we consider the (extremised) collectiveness within the Maoist value system, as well as the emphasis on the notion of exemplar in Maoism. ${ }^{30}$ YAN Yunxiang has recently examined the process of individualisation in contemporary China, and he concludes that the Chinese path to individualisation is, indeed, different from the path(s) of European and North American; and, that the Maoist revolution has led to what he calls a "partial and collective type of individualisation" (YAN 2010, pp. 493-494).

YAN points out that the Chinese notion of the individual (or, the self) is best understood in duality consisting of a small self (Xiaowo 小我) and a great self (Dawo 大我): whereas the small self concerns with personal interest, the great self concerns with the country's interest. Here, the great self appears to exemplify the essence of the Confucian value system. Similar to youhuan yishi, the great self has a priority over the small self. In other words, the Chinese concept of the individual entails that autonomy has to be understood in and under the context of country, but not above it. In short, Confucianism remains influential in thinking about the concept of the individual as an acting and knowing subject; and, it also has shaped and proffered a different meaning of autonomy unlike liberal autonomy.

\subsubsection{Zhongyong as Confucian Rationality}

Let's now turn to mastery. Mastery refers to the way in which human beings understand and interact with the world. In European and North American modernity, mastery is understood in light of rationalisation, i.e. a process of disenchantment of magical, supernatural and religious ideas and their replacement by scientific ideas as well as an orientation towards efficiency. JIN Guantao (1997) argues that China had undergone a different type of rationalisation which, unlike the West, does not rest on science nor aims at efficiency (see also, JIN \& LIU 2009, Chapter 1). He notes that the Chinese rationalisation is characterised by a process of rationalising common knowledge. Accordingly, the intelligibility of the world is not necessarily grounded in science, but in instinctive sentiments and/or common

\footnotetext{
30 For a discussion of the structural and substantial similarities between Maoism and Confucianism, see Davies (2010) and YAN (2010).
} 
sense. Moreover, the Chinese rationalisation is oriented towards (moral) perfectibility, instead of efficiency. Hence, there is not a clear separation of instrumental rationality and value rationality. He concluded from the differences between the two types of rationalisation that China and the European and North American countries have two distinct understandings of rationality (JIN \& LIU 2009, p. 42; JIN 1997). Still, it is true that from the 1950s onwards, the Communist China has become increasingly reliant on scientific ideas and oriented towards efficiency. But, as JIN rightly notes, the fundamental structure of rationality for common people in China has not been thoroughly transformed into the Enlightenmentinspired rationality; the scientific ideas and the orientation towards efficiency are accepted only insofar as they become part of common knowledge (JIN \& LIU 2009, pp. 69-70).

Perhaps, a more interesting and direct way to spell out the differences between the two ways of understanding rationality and the role on Confucianism is through the notion of zhongyong rationality. Recently, CHEUnG Tak-Shing and his colleagues $(2003,2006)$ have explicated a notion of rationality presently at work in Chinese societies, which originates from Confucianism and belongs distinctively to the Chinese culture; and, they named it after the Confucian classic The Doctrine of Mean (Zhongyong 中庸). As CHEUNG and his colleagues explain, zhongyong rationality is characterised by two dimensions. First it aims "to find an optimal point of balance between extremes" (CHEUNG et al. 2003, p. 115). Second, a person is disposed to "view oneself as embedded in a social system, and therefore to see things holistically and in social terms" (CHEUNG et al. 2003, p. 116). In other words, zhongyong rationality is based on the notion of harmony (or harmonisation) as I have elaborated in Section 4.2.2. Based on this understanding of rationality, mastery is not to be understood purely in terms of control vis-à-vis instrumental rationality, but in terms of accommodation and optimisation with respect to various constraints and situational factors.

\subsubsection{Towards the Confucian Good Life}

Admittedly, I have not provided a comprehensive defence of the significance of Confucianism in the present day. I believe, however, I have show that Confucianism remains an important influence in interpretations of, responses to and experience in modernity, and thus in the Chinese mode of self-interpretation and self-understanding and, relatedly, its view of the good life. Here, I offer a philosophical-psychological account of the Confucian view of the good life, and 
support it with two empirical studies on the good life (or, the quality of life) in Chinese societies.

\subsubsection{Confucianism and the Good Life: A Philosophical-Psychological Perspective}

All three major notions in Confucianism, i.e. Dao, harmony and Confucian personhood, have given rise to specific characteristics of the Confucian view of the good life. For instance, recall the notion of Dao. Dao is the normative ideal in Confucianism. As I have pointed out, it has priority over the right and the good, and does not distinguish between them. Accordingly, the good life in Confucianism is also the moral life, as the good life and the moral life are unified under the umbrella of Dao. Moreover, Dao, as a normative ideal, is manifested in harmony (and, harmonisation). Hence, the good life has to be conceptualised in terms of harmony; or, to put it differently, in Confucianism, living well involves harmonisation with oneself, one's family, one's community, and one's country. In this respect, the good life in Confucianism is essentially socially oriented, because harmonisation always takes into consideration factors beyond oneself. As David Wong (2006) fittingly labels Confucianism as a community-cantered morality, which is directed at the common good. Finally, the moral dimension and socialorientedness of the Confucian view of the good life is also related to its view of personhood. As previously pointed out, the Confucian notion of personhood is relational, developmental and virtue-based. Hence, being a proper person has already presupposed taking into account the others, especially the family and community members. Also, being a proper person requires individuals to ceaselessly cultivate their own virtues. ${ }^{31}$ In other words, living well is synonymous to living virtuously.

To highlight the uniqueness of the Confucian view of the good life, I shall now compare it with the three theories of the good life I have previously discussed, i.e. hedonism, desire theories and objective list theories. Immediately, it should be obvious, unlike the Confucian view of the good life, that the three theories maintain a conceptual difference between the good and the right, and that they are

\footnotetext{
${ }^{31}$ It should also be recalled that the Confucian virtues are essentially social; in particular, they are informed by a person's social roles. It, thus, provides a notable difference between Confucianism and its view of the good life from other philosophical traditions that also emphasise virtues as a necessary criterion of the good life but do so from an individualistic perspective.
} 
solely about the good. More importantly, I think, the Confucian view of the good life cannot be categorised as any of them. For instance, while the Confucian view of the good life is not intrinsically against pleasure or satisfaction of desires, it does not aim at pleasure-seeking or desire satisfaction. Since it requires individuals to take into account considerations beyond oneself in light of harmony, it may even require individuals to sacrifice their own pleasure and desire satisfaction. In this respect, the Confucian view of the good life is closer to objective list theories. Still, objective list theories, like hedonism and desire theories, appear to aim at maximising certain objective goods. The Confucian view of the good life, therefore, differs from them in that it does not seek maximisation, but accommodation and optimisation; and, accordingly, some goods in the objective list may need to be surrendered in light of harmony. It also entails that individuals ought to be focused on what they already have, and not to look for something new. Moreover, all three theories appear to maintain a predefined good life and conceive the good life as an end state: in hedonism, good life is the maximisation of pleasure; in desire theories, it is the maximisation of desire satisfaction; and, in objective list theories, it is the maximisation of the goods in the objective list. Unlike them, the Confucian view of the good life does not have predefined goals; this is due to the fact that Confucians see the good life as a process, in which individuals are to accommodate and optimise what is in concrete situations, and to continuously make and remake themselves in response to those situations. In short, the Confucian view of the good life is processual, oriented towards the common good, harmonious and virtuous.

It would be naïve to claim that the Confucian view of the good life is completely negligent to self-interest. As aforementioned, the Confucian view of the good life is not inherently against personal interest. More important, as I have noted already, the Chinese notion of the self has to be understood in duality, consisting of a small self, which aims at personal interest, and a great self, which aims at collective interest. And, personal interest is relegated to the small self, which is deemed inferior to the great self. Hence, it is fair to claim that the Confucian ideal does not include personal interest. However, it should also be noted that the relation between the small self and the great self is dynamic, i.e. it can change in response to the social, cultural and historical circumstances (see, e.g. XU 2009). Still, the duality of Chinese self and its leaning towards social-orientedness are supported by some recent research in psychology.

In a series of studies on the Chinese concept of the self, happiness and wellbeing, LU Luo and her colleagues have argued that the Chinese self is 'bicultural', which consists of both a Confucian-rooted, traditional (social-oriented) self, and a 
modern (individual-oriented) self (Lu 2008a, 2008b; LU et al. 2008). Their findings, therefore, verifies the distinction between the small self and the great self. The traditional (social-oriented) self, in LU's definition, refers to "a connected, fluid, flexible, committed being who is bound to others" (Lu 2008a, p. 348). This definition should be familiar, since it coincides with the Confucian notion of personhood I have already discussed. What is more interesting in their research, however, is that they have also shown that the traditional (social-oriented) self is accompanied by a social-oriented conception of subjective well-being, which is characterised by a distinct emphasis of role obligations and dialectic balance (LU 2005, 2008b; Lu \& Gilmour 2006).

As LU (2008b) explains, an emphasis of role obligations in well-being means that happiness is about the fulfilment of social role obligations and its accomplishment through self-cultivation. This emphasis on role obligations, as it should be expected, is tied to the interdependence and relationality in the Confucian notion of personhood. It also reinforces the point that the Confucian view of the good life is essentially oriented towards the common good. By dialectic balance, LU refers to the view that well-being is conceived to include both happiness and unhappiness; or, as LU has put it, "[h]appiness is dependent on unhappiness, while unhappiness is hidden in happiness" (Lu 2008b, p. 296). This dialectical balance in the concept of well-being has exemplified the notion of harmony in that importance is being placed, not on maximisation of one thing, e.g. happiness, but on accommodation and optimisation of both the positive and negative attributes.

\subsubsection{The Good Life in Contemporary Chinese Societies: An Empirical Review}

To sum up, the contemporary Chinese view of the good life has to be understood as a dynamic composition of the Confucian-rooted, traditional (social-oriented) view of the good life and the modern (individual-oriented) view of the good life. Also, as Lu et al.'s findings show, the traditional (social-oriented) view of the good life is still very much alive. Now, to further substantiate my account of the Chinese view of the good life, I shall look at two empirical studies on the view of the good life in Chinese societies conducted by IP Po Keung (2011) and LIU Li (2006, 2008). The reason for looking at their studies is because they aim explicitly to investigate the 
concept of the good life in contemporary Chinese societies; therefore, they provide suitable data to supplement the preceding discussion. ${ }^{32}$

IP (2011) sets to compare the old folk concept of happiness, which is often based on folklore and anecdotes, with the findings of a survey on Chinese people's new folk concept of well-being at personal and national levels. ${ }^{33}$ He argues that the old folk concept of happiness consists of two traditions, i.e. the grand tradition and the small tradition. IP points out that the concept of happiness offered by the grand tradition is drawn principally from Confucianism, and it is considered to be something essentially social (familial), ascetic and educational, whereas happiness in the small tradition is more inclined towards materialistic and non-moral elements, but remained attached to the social (familial) aspects (IP 2011, p. 468). He then compares the two folk concepts of happiness with the findings of a scientific survey, where it is reported that the elements most frequently ${ }^{34}$ included in happiness are: health in body and mind, satisfactory family life, good human relationships, satisfactory work, and satisfactory sex life ${ }^{35}$ and, it is also reported that a significant number of respondents did not include wealth in happiness. ${ }^{36}$ The old folk concept of happiness and new folk concept of happiness, as IP points out, are largely consistent. ${ }^{37}$ Particularly, he notes that both of them have emphasised social and familial aspects, e.g. satisfactory family life and good human relationships.

32 It should be pointed out that the empirical studies I am discussing use the term 'subjective well-being', 'personal well-being', etc. instead of 'the good life'. However, the notion of subjective well-being is, at least, an indicator of a person's overall satisfaction of his life, which is compatible to the notion of the good life I have employed so far.

33 It should be noted that the subjects of IP's study are Taiwanese. But, as he points out, his notion of Chinese is cultural and ethnical, where majority of Taiwan people are considered (and considered themselves) to be Chinese (IP 2010, p. 461).

34 The rate of selecting those items is $90 \%$ or beyond, with the exception of satisfactory sex life, which is at $83 \%$.

35 Compare the other items in Table 1.

36 For instance, $65 \%$ of the respondents agree with "more wealth more happiness" in comparison to $90 \%$ or above of the respondents agree with items including "Body mind health", "Good family life", "Good social relationships" and "Good job".

37 IP (2011), however, has argued that there is no corresponding notion of national wellbeing in old folk concept of happiness. This conclusion, however, has overlooked the inseparability of personal well-being and national well-being as well as the fact that social (familial) dimension of well-being is the model of national well-being. 
Table I

\begin{tabular}{|l|l|}
\hline Selected samples & - Free dental services for people over \\
of additional & - Understand one's own self \\
personal well- & - Has a definite life goal and values \\
being & - Happiness of all produces one's happiness \\
- Be kind to others, optimistic, be grateful, mutual \\
- understanding and trust \\
- Do not be mutually suspicious of each other \\
- Have three generations under one roof \\
- Children to meet parent's expectation \\
- Children stay healthy \\
- Have good children \\
- Have opportunity to develop one's talents \\
- Unity of spirit between husband and wife \\
- Peace of mind \\
- Rich in spirit \\
- Joyful living \\
- Leisure and entertainment \\
- Have a full belly \\
- Do more exercise \\
- Have personal favourable hobbies \\
- Have self control \\
- Knowing one is having enough makes one \\
always happy \\
- Self moral improvement \\
- Free, and having plenty of free time \\
- Respected by others \\
- Peace and joy in mind given by religion \\
- To serve others \\
- Gives back to society \\
- Able to let go \\
- Do what one likes to do \\
- Buy what one wants to buy
\end{tabular}

Note. Adapted from "Concepts of Chinese Folk Happiness", by IP Po Keung (20I I), Social Indicators Research, I04 (3), pp. 47I -472.

However, he also notes that the new folk concept of happiness is more individualoriented, focusing on health, material life, etc. and also does not have a strong moral dimension in it (IP 2011, pp. 468-469).

IP concludes that the new folk concept of happiness has less resemblance to the concept of happiness in the grand tradition. But, we may question IP's conclusion, especially his claim that the new folk conception of happiness does not have a strong moral dimension because the studies have distinguished personal well-being from national well-being. In the Confucian tradition, the distinction of the private and the public cannot be made easily. Hence, we may question if the moral dimension of well-being has already been captured in the separated notion of 
national well-being. Indeed, it is clear that the folk concept of national well-being in IP's study is directed at the common good. ${ }^{38}$

Let's now turn to the studies by LIU Li (2006 \& 2008), who examines how (subjective) well-being is conceptualised by the Chinese people through openended individual interviews. ${ }^{39}$ From the interviews, he derives that well-being is conceived in terms of a logic of 'having' and a logic of 'being', where the former gives priority to possession and consumption of materialistic elements, e.g. money, food, housing, and car, the latter is about existential merits and spiritual subsistence, including natural and ancestral rootedness, connectedness, participation, and freedom (L. LIU 2008, pp. 238-246). ${ }^{40}$ It is apparent in LIU's analysis that personal interest, social and familial aspects as well as the common good are all constituents of well-being as it is conceived and expressed by the respondents. More intriguingly, however, is LIU's analysis of the relation between the logic of 'having' and the logic of 'being'. He points out that in the logic of 'having', possession and consumption of materialistic elements are considered to be the goal of living, and the existential merits and spiritual subsistence are merely subsidiary; but, in the logic of 'being', existential merits and spiritual subsistence are prioritised, and materialistic elements are regarded as entirely instrumental (L. LIU 2008, p. 238). Setting aside the details of LIU's analysis, the logic of 'having' and the logic of 'being', I think, clearly reflect the duality of the Chinese self, i.e. the small self and the great self; and, well-being, as it is conceived and expressed in the logic of 'being', is one that closely resembles the great self, that is-the Confucian view of the good life.

\footnotetext{
$3^{8}$ For example, the most frequently selected items are: having no wars or threats of war; having good law and order, and people's life and property was protected; having the economy in good shape; people's standard of living improved; having harmony between ethnic groups; having equality for all, and human rights protected; having clean and effective government that cares for people's livelihood; having a judiciary that is fair and independent; having no big gap between the rich and the poor; having a good health care system, people's health is taken care of; having a good education system with equal opportunity in education; having the natural environment protected; having people trusting each other (IP 2011, p. 5).

39 Cf. LU (2001).

40 As LIU Li points out, the term 'freedom' is not intended to refer to political freedom but "the individual's emancipation from interference or restrictions by the State [and] a kind of bestowal derived from, granted or conceded by, the State" (L. LIU 2008, p. 246).
} 
In this section, I have discussed two empirical studies on the view of (subjective) well-being. IP's quantitative study shows that the view of the good life in contemporary Chinese societies has emphasised social and familial aspects, which is consistent with the Confucian tradition; and, LIU's qualitative study has reflected the duality of the Chinese self, and the two views of the good life of the small self and the great self. It should be recalled, however, that the discussion in this section is descriptive, and it does not aim to arrive at a normative conclusion, e.g. to claim that people should adopt the Confucian view of the good life. To restate the purpose of this section, it aims to describe and elaborate the empirical studies on the Chinese view of the good life and to see if the Confucian view of the good life is present in the view of the good life actually held by the Chinese people. In doing so, I hope it has established that the Confucian view of the good life is indeed a legitimate resource for thinking about issues related to the good life.

\subsection{Conclusion}

The main objective of this chapter is to identify an alternative view of the good life, i.e. the Confucian view of the good life. In the first part of this chapter, through a detour to modernity, I argue that if the views of the good life are based on our selfinterpretation and self-understanding, which, in turn, are rooted in specific social, cultural and historical backgrounds, then the views of the good life I have identified in Chapter Two are insufficient for normative analysis of digital media and the good life in an intercultural context, because they are originated from the European and North American cultures.

In Section 4.1 and onwards, I examine the view of the good life from a different tradition, i.e. the Confucian tradition. To do so, I discuss three major notions in Confucian thought, namely Dao, harmony and personhood. Using these notions, I outline a basic account of Confucian ethics, which supports a theory of the good life that is different from the three notable families of theories of the good life in current philosophical discussion, namely hedonism, desire theories and objective list theories. Then, I offer an account of the Confucian view of the good life as one that is virtuous, harmonious, oriented towards the common good and processual. Finally, I support this account with two empirical studies on the concept of the good life in contemporary Chinese societies. 


\section{CHAPTER FIVE}

The Making of China's Internet

The Walzerian Approach in an Intercultural Context

From 2010 onwards, a series of events have illustrated the Chinese Communist Party's (CCP) position on the Internet and its ostensible differences from the prevailing position(s) in European and North American countries. For instance, in the beginning of 2010, Google threatened to withdraw from China over issues regarding Internet censorship and alleged cyber-attacks from the Chinese government, and Google's actions were seemingly backed by the US government, e.g. Hillary Clinton's speech on "Internet Freedom" (Clinton 2010). In return, the CCP responded by criticising both Google and Hillary Clinton, and then, in mid2010, the CCP published a white paper, entitled The Internet in China, outlining its Internet policy (State Council Information Office of the People's Republic of China [SCIO] 2010). These events have illustrated two points. First, the Chinese government has become increasingly active in promoting its own position on the Internet in the globalising world, and it involves not only a defensive response to its international observers and critics, but also a substantive illustration of its own position. Second, while similar terms such as 'rights' and 'freedom' are being used by the CCP and the West in stating their positions on Internet-related issues, the clashes between the two sides have indicated that the two positions are clearly distinct.

Although there is clearly an interesting contrast between the CCP's and the European and US's positions on the Internet, and the European and US's views on 
have received much attention from researchers, relatively little attention has been paid to the CCP's position. ${ }^{1}$ It should be reminded that even if it is often too tempting to disregard the claims on the Internet made by the CCP as merely propagandistic, they nonetheless present a different way to perceive the benefits and harms of the Internet. More importantly, insofar as its way to perceive the Internet expresses some normative judgements on the impacts of the Internet on people's lives and envisions some relations to the Internet as more preferable, it (re)presents another way to look at the relations between digital media and the good life. In short, if the CCP's position on the Internet is based on a distinct normative and axiological foundation, it will be unwise to deny its position outright.

This point is well recognised by the Walzerian approach to digital media and the good life, which rejects the idea that there is an impartial, objective and universal normative standard and insists that normative judgements ought to be made using values of the community. In this respect, this chapter can be viewed as an exercise of applying the Walzerian approach in an intercultural context. In short, the main objective of this chapter is to provide a normative analysis of China's Internet using its own view of the good life and values.

In this chapter, I examine the axiologisation (i.e. what benefits and harms are being foregrounded) and problem representation (i.e. what problems are being represented) of the Internet in two document sets on the Internet and Internetrelated activities, namely Internet policy documents and a set of opinion pieces in Guangming Daily (光明日報), and illustrate the normative and axiological foundation of the CCP's view of the Internet. I show that its view is fundamentally Confucian in nature. This, I then argue, has an important implication to our

\footnotetext{
1 As MENG (2010) points out, research on China's Internet is often dominated by what she calls the democratisation perspective, in which the Internet is investigated for its potential for democratisation and for its (possible) influence on the political authority of the Chinese government. Of course, the topics from the democratisation perspective are important. However, as MENG rightly notes, such a perspective risks overlooking other equally important topics that are not immediately related to democratisation, e.g. the good life. Moreover, from the democratisation perspective, which is essentially based on the Euro/Ameri-centric standard, it is all too easy to dismiss any claims made by the Chinese government that fail to satisfy such a standard as illegitimate. Hence, in order to interrogate the CCP's position honestly, a different perspective is needed. This perspective should take into account the fact that the Chinese government has (or can have) its own normative and axiological foundation. For a similar argument, see Herold (2011).
} 
assessment of China's Internet; particularly, we should reconsider the oft-heard liberalist critique of China's Internet and see it in a different light. Finally, I provide an example of how it can be done by investigating the potential conflicts between Confucian values and social media.

Before proceeding, it should be noted that I am using the CCP's view of the Internet as a case study to demonstrate how intercultural normative analysis of digital media and the good life can be done, and the present chapter is not intended to be a comprehensive study of the impacts of the Internet on the lives of Chinese people in general. Hence, the focus here is on the view of the Internet maintained by the CCP, but not by the Chinese people in general. It is, indeed, plausible that the people in China do not (fully) agree with the CCP's view of the Internet, as well as its normative and axiological foundation. However, a comparison between the CCP's view of the Internet and its normative and axiological foundation and those held by the people in China belongs to the scope of an intra-cultural study, which is not focus of this chapter. ${ }^{2}$ To reiterate the objective of this chapter, the aim is to apply the Walzerian approach at a global level.

\subsection{The Chinese Communist Party and the Internet}

The CCP has expressed its view on the Internet and Internet-related activities on various occasions and to both internal and external parties. To its internal audiences, it has expressed the vision on the development and use of the Internet; and, to its external audiences it has responded to the critics of its Internet policy. In particular, the CCP has vigorously reacted to the Google incident in 2010 by outlining and asserting its view on the Internet. The official documents, therefore, offer a vivid documentation of the CCP's position at the policy level. Here, I shall draw on two official documents in my analysis of the CCP's position on Internet, i.e. the report on Concerning the Development and Administration of Our Country's Internet (WANG Chen 2010) and the white paper on The Internet in China (SCIO 2010). My choice of the two documents is not arbitrary. It is based on the fact that they are directed at two different groups of audiences, namely the party officials and the

\footnotetext{
2 An intra-cultural normative analysis of digital media and the good life in the Chinese context also requires a more in-depth study of the notions of the self prevail in contemporary Chinese societies. Although this project is an interesting and important one, it is well beyond the scope of the present study.
} 
international observers and critics. In this respect, they offer an overview of the CCP's position on the Internet to both internal and external parties.

At the same time, the Internet (and Internet culture) is also being discussed in (official) newspapers in China. Particularly relevant to the present study is a series of articles in Guangming Daily from May to June 2010, which discusses the potentials and problems of the Internet and Internet-related activities in China. Since the intended readership of Guangming Daily is the local (educated) people, the series presents a view of the Internet and Internet-related activities at the social and cultural level. Together, the two document sets provide us with a reasonably comprehensive picture of the CCP's position on the Internet.

\subsection{Governing China's Internet}

At the policy level, the CCP's position on the Internet can be subsumed under the topic of Internet governance, and it is structured around three themes, namely Internet Development, Internet Administration and the Sovereignty of China's Internet. The CCP's concerns with development, administration and the sovereignty of the Internet are obvious, as the title of WANG's report Concerning the Development and Administration of Our Country's Internet illustrated, where the terms 'development' and 'administration' are explicitly mentioned in the title. 'Our Country's Internet' signifies a geographically and/or socio-politically bounded view of the Internet, and thereby suggests the Internet to be a space (or an entity) legitimately under the CCP's rule. The three topics-development, administration and sovereignty-are also repeated in the white paper The Internet in China as well. ${ }^{3}$ In the following, I shall elaborate why they are present in the documents (and how they are presented in them), and how to make sense of them.

\subsubsection{Internet Development}

The CCP recognises two aspects of Internet development, i.e. the technical aspects and the sociocultural aspects. Yet, Internet development is represented mainly to be a technical problem. For instance, in the list of actions proposed in the report to, "further promote the scientific, healthy, and orderly development of our country's Internet" has an overt emphasis on technical aspects of the Internet such as

\footnotetext{
3 Although the report and the white paper are two different official documents, they are very similar in content (and, to a certain extent, their structures as well). A possible explanation for their similarity is that they are likely to be prepared by the same bureau.
} 
"Internet application structures", "Internet applications", “content building”, "web application system", and "core Internet technologies" (WANG 2010). The same emphasis on technics is visible even in the report's discussion of sociocultural aspects of Internet development. As it is stated in the report, the CCP has to "develop a web culture with Chinese characteristics, to provide more and better web cultural products and services for the people and ensure that positive and healthy web cultural products occupy the leading position on the Internet" (WANG 2010). The development of "a web culture with Chinese characteristics" is purported to be achieved through technical means such as construction of information infrastructures (e.g. a digital library, a national knowledge resource database and a public cultural information service system), digitisation of traditional and local cultural resources, and the transformation of traditional (cultural) industry into an online, Internet (cultural) industry. In short, the choice of inclusion in the report as well as its choice to exclude other aspects of China's Internet development, suggests that Internet development is represented to be a technical problem concerned with Internet infrastructures and technologies (WANG 2010). ${ }^{4}$

The CCP's representation of Internet development as a technical problem is consistent with its impact view of the Internet (Damm 2007, pp. 279-281). ${ }^{5}$ In effect, the instrumentalist attitude towards the Internet is further illustrated by the emphasis on the actual and potential socioeconomic and political benefits brought by the Internet in the report. ${ }^{6}$ It is, therefore, possible to question whether this instrumentalist attitude towards technology is in itself tenable. However, setting aside the question about the adequacy of the CCP's impact view of the Internet, the

4 This is also true in the white paper as well. For instance, section I of the white paper, entitled Endeavors to Spur the Development and Application of the Internet, is preoccupied by a description of the development of China's Internet infrastructures and technologies.

5 For the discussion of various views of technology, see Chapter 1, section 1.4.2.2.

6 For example, it is emphasised in the report that the Internet has "advanced the adjustment of [China's] industrial structure and the transformation of [its] economic growth model", "increased the [Chinese] government's capabilities in social management and provision of public services", "opened up and broadened the channels that connected [the Chinese government] with the masses", "broadened the front of our propaganda and ideological work" and become "an important vehicle of advanced socialist cultural development" (WANG 2010). The same emphasis on instrumental goodness, i.e. socioeconomic and political benefits, of the Internet can also be seen in the white paper, especially in II Promoting the Extensive Use of the Internet. 
list of actions concerning Internet development has clearly illustrated that the CCP regards the Internet to be good, even if it is only instrumentally so. For instance, it is stated in the report, the Internet "has become an important engine that drives economic development, an important infrastructure for the operation of modern society, and a key area of international competition" (WANG 2010).

As the quote above illustrates, the good of the Internet is aimed at the country and at the level of the common good. It is worth noting that the actual and potential benefits of the Internet are often framed in the report using non-personal and non-individualistic terms with China's economy, government, society and culture as the beneficiaries of Internet development. The Internet and its impacts, in other words, are evaluated with reference to the common good. Here, the foregrounding of the Internet's actual and potential benefits to the common good echoes the Confucian view of the good life, which has prioritised collectiveness and the common good over individual subjects and personal interest.

Of course, the people, i.e. the individual subjects, are not entirely neglected in the documents. Insofar as the benefits brought by the Internet are also conferred to the people, they are also mentioned as one of the beneficiaries from China's Internet development. Yet, the benefits conferred to the individual subjects are often framed as intermediaries to the country's well-being or as the good at the collective level. For example, the introduction and application of the Internet have "facilitate[d] people's work and life", but it is only a derivative of "improve[d] efficiencies of governments at all levels and the degree of openness of government affairs" (WANG 2010). Similarly, the Internet has allowed the people to voice their opinions and raise important issues, but it is for the purpose of "provid[ing] a convenient and quick channel for the Party and the government to understand social conditions and the popular will, and to stay closely connected with the people" (WANG 2010). At this juncture, it seems appropriate to recall the notion of the duality of the Chinese self. As previously noted, personal interest, i.e. the small self, is not entirely rejected in the (modern) Confucian self, but it is often-if not always—overshadowed by the interest at the collective level, i.e. the great self. ${ }^{7}$

7 Since the report aims to provide a vision of Internet governance to party officials, what is at stake is essentially national-the appeal to the country's well-being and/or the good at the collective level is in this respect predictable. In effect, there is more discussion on the benefits the Internet can bring to individual subjects in the white paper. However, the general pattern to prioritise the country over individual subjects and to 
The report has also emphasised the role and importance of Internet in "helping [the government] fulfil people's wishes, meet their needs, and protect their interests" (WANG 2010). This quote, as with the previous paragraph, epitomised the view that the goodness of the Internet is derived from its capacity to enable the Chinese government to fulfil its role obligation. Accordingly, it is the role of the Chinese government to fulfil people's wishes, meet their needs and to protect them, which signifies a (need for a) proactive stance towards Internet governance. So construe, however, it also presumes that people are in need of help to fulfil their wishes, meet their needs and be protected (of their interests). At the same time, the languages of "wishes", "needs" and "protection" have relegated the people to a subordinate position. People are thus framed as inferior in need of their superior, i.e. the Chinese government, which implies that the Chinese government(metaphorically) being the superior - is in a position of moral authority and moral superiority and, therefore, is responsible for determining what is good or bad about the Internet. As I have pointed out in Chapter Three, this image of (Internet) governance is not new in the Chinese context. Confucianism has long portrayed political relationships in hierarchical terms, e.g. familial relationships. In this respect, the CCP can be seen to have inherited the political language from the Confucian tradition.

\subsubsection{Internet Administration}

The Internet is being viewed by the CCP to be instrumentally good, and its goodness depends on the right use of it. This view of the Internet naturally calls for management to ensure that it is being used in the right way. As the report states, the development and administration of the Internet always go hand in hand: Internet administration allows the Internet to develop properly, but the development of the Internet leads to new problems and challenges, which require further management of it. For instance,

[Information in] multi-media forms... [has] increased the difficulty in the supervision of information content security. [Internet use] is gradually becoming more deeply and broadly entrenched in the national economy. [...] [T]his has set a higher demand for building Internet integrity. [...] Internet is expanding from providing information to providing platform

frame the benefits in terms of country well-being and the good at the collective level still remains in the white paper. 
(i.e. interactive) services. [...] Internet users are not only consumers of information, but also its providers and creators. This has [...] set a higher demand on Internet users to abide by the law and learn to discipline themselves. [...] Traditional Internet is expanding to mobile Internet. [...] This has [...] but set a higher demand for regulating the orderliness of online information dissemination. (WANG 20I0)

Four new problems and challenges are listed in the report, and it is pointed out that they need to be overcome if the Internet is to deliver any (instrumental) good. In presenting the problems and challenges, there is again a recurring absence of individual subjects in the discussion. "Supervision of information content security", "building Internet integrity", "abid[ing] by the law and to disciplin[ing] themselves [on the Internet]" and "regulation of online information dissemination" are measures to be done for the society-at-large. It has illustrated the weigh being placed on the collective, i.e. the problems and challenges from the Internet are presented as collective issues, and concerns from personal and individual perspectives are silenced in the report. To this point, the view on users' legal and moral responsibility (i.e. "demand on Internet users to abide by the law and learn to discipline themselves") is particularly telling. Here, Chinese Internet users (i.e. the individual subjects) are represented as one of the problems and challenges. This final point has reinforced the view that the CCP has presumed a morally authorial and superior position than the average Internet user as well as the importance of role obligation in determining the goodness of the Internet.

The previous point also connects to another convergence between the CCP's view on the good of the Internet and Confucianism, namely the unity of the right and the good. Accordingly, the Internet is good only insofar as it is being used in the morally right way; in effect, as it is stated in the report, China's Internet "has flourished" because of "the correct leadership of the [CCP]" (WANG 2010). Likewise, the deficiencies of the Internet are the results of immoral uses. For instance, the problems and challenges of the Internet are represented in the report as moral problems of "harms" and "threats" to the common good, i.e. country's well-being and the future generation, e.g.

Internet security problems are becoming more conspicuous [...]: Online harmful information such as pornography and obscenities are seriously harming the physical and mental health of minors. Criminal activities such as online fraud and theft are seriously harming public security. Computer viruses and hacker attacks are posing serious threats to the security of the operation of the Internet. Leaking of secrets via the 
Internet is posing serious threats to national security and interests. (WANG 20I0)

\subsubsection{Sovereignty of China's Internet}

So far, I have mainly focused on the CCP's portrayal of the Internet's benefits and harms in the report. In terms of content and structure, the report and the white paper are very similar, but, they are directed at very different audiences. As I have pointed out, the report aims to articulate a vision of Internet governance for the party officials, whereas the white paper is aimed at legitimatising the CCP's Internet policy and responding to the International observers and critics. Here, one of the major criticisms to the CCP's rule of the Internet has been framed using the language of rights. In the white paper, the CCP has attempted to use the same language to reply to its observers and critics; therefore, "individual subjects" and "rights" are notably more visible in the white paper than in the report.

Using the language of rights, the CCP aimed to justify its rule of the Internet in China and to respond to the criticism that the Chinese Internet users do not enjoy the same (human) rights as the Internet users in Europe and the United States do. To the observers and critics of the Chinese Internet, the title of the third section Guaranteeing Citizen's Freedom of Speech on the Internet may appear hypocritical or, at least, paradoxical. In effect, it is stated that:

The Chinese government encourages and supports the development of Internet news communication undertakings, provides the public with a full range of news, and at the same time guarantees the citizen's freedom of speech on the internet as well as the public's right to know, to participate, to be heard and to oversee in accordance with the law. (SCIO 2010)

Those "rights" are given another meaning in the white paper. For instance, the right to know is associated with "authoritative news", and freedom of speech is linked to the opportunity to "voice [...] opinions in various ways of the Internet" (SCIO 2010). Expression of "opinions", however, is mainly represented by quantity, namely the number of channels (e.g. Bulletin Board Systems, Blogs, etc.) Chinese Internet users have, as well as the amount of information created and shared on the Internet. What have not been discussed in the white paper are the types of expression of "opinions" on the Internet. Particularly, the idea of freedom of political expression is conspicuously missing in the white paper. As Min JIANG (2010) 
points out, political rights are limited in China throughout its history and, in accordance with Confucianism, socioeconomic justice is more important in the Chinese context historically (see also, Perry 2008). In the white paper, the representation of rights clearly follows the priority of the socioeconomic rights over the political rights. Hence, it is not an accident that the white paper has foregrounded those issues related to accessibility of the Internet, e.g. Internet infrastructures and technologies, channels of expressing opinions, etc. As the Internet is being viewed as an essential tool for the people to flourish socially and economically, it is the CCP's duty to provide that tool to the people. Hence, accessibility to the Internet becomes the main focus in light of the importance of socioeconomic rights. The provision of the tool itself, however, does not preclude any restrictions on its applications, especially when the Internet threatens the country's social, political and economic orders. As the white paper stated, although “[Chinese] citizens' freedom and privacy of correspondence is protected by law, [...] exercising such freedom and rights [cannot] infringe upon state, social and collective interests or legitimate freedom and rights of other citizens" (SCIO 2010).

It should be pointed out that the political potential of the Internet is also mentioned, e.g. "[ $t]$ he Internet's role in supervision", "[t]he internet $[a s]$ a new channel for the Chinese government to get to know the people's situation and amass the public wisdom", etc. (SCIO 2010). The discussion of the political potential of the Internet is, however, linked to fortification of the Chinese government's legitimacy. For instance, "supervision" is not framed as a mechanism of checks and balances - or, in other words, a form of resistance to the government itself-but as a mechanism to facilitate the existing orders, e.g. the Internet enables the people to report problems of corruptions and degenerate officials. In short, there is an emphasis on the importance of non-conflict and stability in the white paper.

\subsubsection{Confucianism and an Internet with Chinese Characteristics}

In the previous sections, I have attempted to demonstrate the presence of a Confucian outlook in the CCP's presentation of Internet governance. Particularly, I have pointed out that there is an overt emphasis on the (instrumental) good of the Internet being directed at the common good (and the country's well-being) and it is prioritised over the good of the Internet for the individual subjects, which coincides with the social-orientedness of the Confucian view of the good life. At the same time, I have illustrated that the good Internet is equivalent to the moral Internet, where the government is responsible to maintain its moral standing. Yet, it is not to say that individual subjects are entirely irrelevant in the discussion of the good of 
the Internet in the context of China's Internet. But it does imply that in this view, it is the task of the Chinese government to ensure the Internet is being used (morally) right so that the country and its individual subjects can flourish with it.

In the next section, I shall further support my interpretation by looking beyond the policy documents, and analyse the discussions of the Internet for and from the Chinese public. In ending this section, I want to point to a more explicit and less controversial presence of the Confucian perspective in the CCP's presentation of Internet governance; namely its use of the rhetoric in Confucianism. Rhetorically, it is relatively clear that both the report and the white paper use the values inspired by Confucianism to frame the issues. Familiar normative concepts in Confucianism such as "well-proportioned", "social harmony" (or, simply, "harmonious"), "healthy", and "stability" are frequently used to elaborate the objective of development, administration, and maintenance of sovereignty of China's Internet. These concepts can be generalised in the idea of harmonious Internet. While it is true that what a harmonious Internet stands for is not clearly articulated by the CCP in the documents, together with its frequent conjunction with "Chinese characteristics", it is clear that the CCP intended to frame a view of the ideal Internet and Internet culture using the lexicon of Confucianism.

\subsection{China's Internet for the Public}

To some extent, the focus on the common good in the CCP's policy documents is predictable, because they aimed at providing directives to the party officials for governing the Internet, and to justify its Internet policy to the outsiders. Both of them are issues directed at the country, i.e. China. As the subject in the policy documents is the country itself, it should be expected that individual subjects are relatively unimportant in the discussion. In order to obtain a more comprehensive picture of the appraisal of the Internet in China, it is necessary to go beyond the policy documents and to look at the discussion about the Internet in the public. Here, I will look at a series of articles published in Guangming Daily, focusing particularly on how the Internet and related phenomena are being axiologised and represented in the articles.

\subsection{1 "Aspects of Network Culture": An Overview}

From May to June 2010, Guangming Daily has published a total of fifteen articles on various topics about China's Internet and other related phenomena. The topics were 
Table I

\begin{tabular}{|c|c|c|}
\hline $\begin{array}{l}\text { Headlines in the } \\
\text { series “Aspects of } \\
\text { Network Culture" } \\
\text { (網絡文化面面觀) }\end{array}$ & $\begin{array}{l}1 . \\
2 . \\
3 . \\
4 . \\
5 . \\
6 . \\
7 . \\
8 . \\
9 . \\
10 . \\
13 . \\
12 \mathrm{~B} \\
11 . \\
12 \mathrm{~A} \\
9 .\end{array}$ & 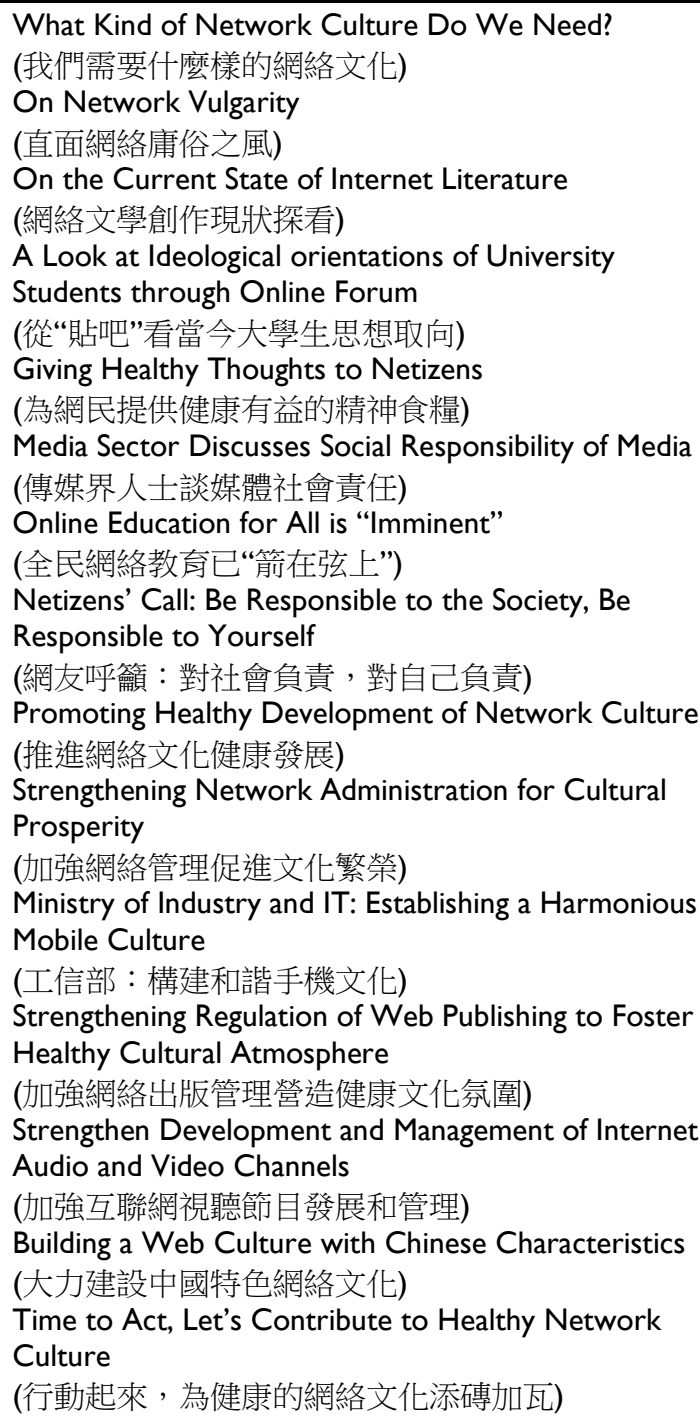 \\
\hline
\end{tabular}

Note. From "Aspects of Network Culture” in Guangming Daily, 13 May 2010 17 June 20 I0. Available Online at: http://www.gmw.cn/content/node_8562.htm

discussed from a macroscopic level (see, Table 1, e.g. [1], [2], [5], etc) and from a microscopic level, looking at specific Internet-related phenomena in China (see, Table 1, e.g. [3], [4], [11], etc). The series of articles is composed mostly of 
interviews and dialogues with media professionals, academics and officials in China, as well as comments from (selected) Chinese netizens. In this respect, the series helps illustrate how the Internet is being axiologised and represented to the public. ${ }^{8}$ In the series of articles, the Internet is always conceived as both beneficial and harmful. For instance, as the opening article of the series stated, "the network culture has its positive and healthy (積極健康) side, but it also has an ambivalent (魚龍混雜, 泥沙俱下) side, it even contains some vulgar cultural rubbish (低俗的文 化垃圾)" (Guangming Daily, [1]). While it is clearly acknowledged in the series that the Internet is (potentially) beneficial to the people, the emphasis is often being placed on the negative impacts of the Internet. As Marcella Szablewicz (2010) noted, the public discussion of the Internet in China can be viewed as an instance of moral panic. ${ }^{9}$ The Internet is often portrayed as a corrupting force to the Chinese culture and has to be countered by various measures. In this respect, both the representation of the problems of the Internet and, more importantly, the proposed answers to the problems in the series are relevant to the present study, as both the problems and the proposed answers have foregrounded the values important in the Chinese context. In effect, one can view the series as offering recommendations to shape an Internet 'with Chinese characteristics', and as some of the headlines suggested, a 'healthy', 'harmonious' and 'culturally prosperous' Internet. In the following, I shall illustrate how the problems and proposed answers are fundamentally shaped by the Confucian outlook.

\subsubsection{Internet Vulgarity}

In the series, a recurring topic concerning the negative impacts of the Internet in China can be summarised readily by Internet vulgarity. In its simplest sense, Internet vulgarity refers to illegal and harmful uses of the Internet as well as illegal and harmful contents available online. Borrowing the mission statement from the China Internet Illegal Information Reporting Centre (中國互聯網違法和不良信息 舉報中心[CIIRC]), Internet vulgarity can be understood as “contents [and uses]

8 The texts discussed in this section are only available in Chinese. Where necessary, I will provide my translation in footnote. While I will try my best to be faithful to the original meaning of the texts, one should be cautious of the possibility that some aspects of the meaning may be lost with translation.

9 Szablewicz's (2010) article focuses mainly on the discussion of Internet addiction in China. However, as I will show in this section, such a moral panic can indeed be generalised to the Internet (and Internet culture) as a whole. 
harmful to the healthy growth of minors, such as obscenity and pornography, gambling, violence, terror, criminal abetting, and contents that spread ethnic hatred, libelling and insulting, violating the others' rights, and violating intellectual property rights." 10 From the mission statement, it is not difficult to discern two major concerns about Internet vulgarity, namely the future generation and the country's interest. ${ }^{11}$

It is true that children, youth and the Internet is not a topic unique in the Chinese context. The impacts of the Internet on children and youth-both negative and positive-are widely discussed in the European and American context too, but the discussions there often focus on risks and opportunities offered by the Internet. Particularly, online risks are often discussed through the image of "child in danger" and "dangerous child" 12 (Oswell 1998, quoted in Livingstone (2011). While the image of "dangerous child" does not receive coverage in the series, the image of "child in danger" can be found in there. However, the danger that arises from the Internet in the Chinese context is predominately discussed with a moral and evaluative overtone. For instance,

"Experts believe that Internet vulgarity has spread erroneous views of life and value system, poisoning the physical and mental health of youth. It must be put to an end, otherwise it will have disastrous outcomes."13 (Guangming Daily, [2])

"At the same time, these vulgar [Internet] culture has a serious influence on people's-especially the youth's-formation of worldview,

\footnotetext{
10 See the website of CIIRC (http://ciirc.china.cn/).

${ }^{11}$ Here, although the language of rights is being used, following the previous discussion of the CCP's interpretation and understanding of rights, it seems safe to assume that the term 'rights' only refers to socioeconomic rights. And, the statement's singling out of intellectual property rights only confirms this assumption.
}

12 As Livingstone explains, the image of a "child in danger" is one that children are seen to have a "'special' status as innocents [which], if [they are] kept separate from the ills of adult society, might bring better prospects for the future." (Livingstone 2011, p. 151: HBK of Internet Studies) On the other hand, the image of a "dangerous child" can be understood as one that portrays children acting against the existing social and cultural norms, and thus posing threats to the society and culture.

13 In CHINESE: 專家們認為, 網絡庸俗之風傳播錯誤的人生觀價值觀, 毒害青少年身 心健康, 此風不鞄, 必將貽害無窮。 
view of life and value system, and becomes the stumbling stone to the healthy development of our country's Internet."14 (Guangming Daily, [9]) "Along with the increased usage and popularity of the Internet, Web publishing has undergone a rapid development. It becomes a major channel to satisfy the people's need in their spiritual life, it also becomes an important base for youth, especially the minors, to develop their mind and morality." 15 (Guangming Daily, [12A])

The quotes above are just a few examples; similar comments can be seen throughout the entire series. There is an overt emphasis on the Internet's impacts on the worldview, morality and values of children and youth, which can be explained by the importance of (self-)cultivation deeply rooted in the Confucian view of the good life. Hence, if the Internet is to become part of daily life, it also has to be viewed as a space for self-cultivation as well. In effect, the stress on the Internet as a space for spiritual and moral life is not only limited to children and youth but also extends to people in general as well. At the same time, the discussions of children, youth and the Internet in the series do not stop at the Internet's impacts on children and youth itself, but are also further extended and connected to the future of the country. In short, the issues about children and youth are not viewed merely as personal, but they are essentially linked to the country's well-being as well.

\subsubsection{Regulation and Guidance}

In order to counter Internet vulgarity and other potential harms from the Internet, it is explicitly pointed out in the series that the Internet has to be regulated. ${ }^{16}$ While the metaphor of Great Firewall of China ${ }^{17}$ has often viewed to have epitomised regulation of the Internet by the Chinese government in form of censorship in the political (and academic) discourse outside China, censorship of the Internet is only

\footnotetext{
${ }^{14}$ In CHINESE: 但與此同時, 網上也出現了一些低俗文化的現象。這些低俗文化, 嚴 重影響著人們特別是青少年樹立正確的世界觀、人生觀和價值觀。

15 In CHINESE: 隨著互聯網的應用和普及, 網絡出版服務也取得了迅猛發展, 不僅成 為滿足廣大人民群眾精神文化生活需要的主要新媒體, 也成為面向青少年特別是未 成年人開展思想道德建設的重要陣地。 
one of the measures discussed in the series. In effect, it has been repeatedly noted in the series that censorship of the Internet alone is insufficient. Instead, responsibility of the people, the media and the Chinese government has been foregrounded in the series as the key to counter Internet vulgarity and other potential harms.

Accordingly, the Internet users, i.e. the people, have to regulate and manage themselves on the Internet because it is a (moral) responsibility to themselves as well as to the common good (see, e.g. Guangming Daily, [7], [8] \& [14]). Likewise, social responsibility of the media has been foregrounded in the series (see, e.g. Guangming Daily, [6], [7], [12A] \& [12B]). Social responsibility of the media, however, does not only amount to (self-)regulation of illegal and harmful content, it also amounts to dissemination of information that is deemed legal and healthy, which is viewed as essential to the country's well-being. In short, the discussions surrounding regulation are mainly concerned with self-discipline. The focus on self-discipline is connected to the previous discussion that the Internet is viewed as a space for self-cultivation. Self-disciplining should be distinguished from merely following the externally imposed laws, as it is an activity performed by the agents from within and its aim is to transform them into virtuous agents. Here, again, morality has been highlighted in the discussions of the Internet, and the question about the goodness of the Internet is essentially a moral one.

At the same time, the importance of the Chinese government in ensuring the spiritual and moral quality of the Internet has also been foregrounded in the series. As I have noted that the discussions on censorship of illegal and harmful information on the Internet only tells part of the story, the series also emphasised the importance of guidance for the people provided by the Chinese government. Here, the purported guidance is not merely about the people's Internet skills, nor is it only about the kinds of information Internet users ought to avoid. Instead, guidance refers to active promotion and diffusion of a specific spiritual and moral view on the Internet (see, e.g. Guangming Daily, [1], [7] \& [13]). In other words, the Chinese government is to proactively shape the Internet into a spiritual and moral space. It is a clear demonstration of both the presumed moral authority and superiority of the Chinese government as well as the importance of spiritual and moral quality of the Internet in determining its goodness. Yet, most interesting, perhaps, is the belief that the guidance provided by the Chinese government can, and should, guide and untie the multiplicity of (cultural) values on the Internet, which illustrates the priority of the pursuit of harmony on the Internet familiar in a Confucian outlook. 


\subsubsection{Exemplary Chinese Netizens}

Finally, the closing article of the series, which address the meaning of 'proper netizens in China', can be viewed as one of the best illustrations of the Confucian outlook underneath the evaluation of the goodness (and badness) of the Internet. As it stated, a 'proper' netizen is one who ought

I. To be a noble practitioner of network morality.

2. To be a (content) creator of healthy network content.

3. To be an advocate of civilised network trend.

4. To be an enforcer of (safe and secure) flow of information.

5. To be a protector of green network ${ }^{18}$ order.

6. To be a promoter of new network culture. ${ }^{19}$ (Guangming Daily, [14])

The importance of the moral dimension of the Internet and of Internet users is foregrounded with images of "noble practitioner", "[healthy] content creator", "advocate of civilised network trend", "[safe and secure information flow] enforcer", "protector [of network order]", "promoter [of network culture]". These images have emphasised moral responsibility of the Internet users themselves as well as their moral responsibility to the society and network culture. In short, the goodness (and badness) of the Internet is determined by the moral quality of its users.

The discussion of the notion of a proper netizen also reveals a fundamental aspect of Confucian view of the good life, namely the importance of being an exemplar-or, being a paradigmatic person (Junzi). Indeed, a proper netizen proposed in the article can be viewed as a version of paradigmatic person in and for China's Internet, who engages in self-cultivation (i.e. "noble practitioner", "[healthy] content creator") and, at the same time, contributes to the common good (i.e. "advocate of civilised network trend", "[safe and secure information flow] enforcer", "protector [of network order]", "promoter [of network culture]").

It should be clear that the judgements of the Internet in the series are based on an instrumentalist attitude to the Internet similar to what has already been discussed

\footnotetext{
18 Here, the term "green network" refers to a pollution-free network.

19 In CHINESE: 1. 做高尚網絡道德的實踐者。2. 做健康網絡內容的創造者。3. 做文明 網絡風尚的傳播者。4. 做網絡信息安全流動的保障者。5. 做綠色網絡秩序的維護 者。6. 做新興網絡文化的建設者。
} 
with respect to the CCP's policy documents. The goodness (or badness) of the Internet, accordingly, is determined by its consequences and the Internet users who produce, via the Internet, those consequences. In other words, the appraisals of China's Internet in the series are ultimately the appraisals of the Internet users themselves. That is if the Internet users are good, then the Internet is good and vice versa. Of course, such an instrumentalist attitude towards the Internet and technology in general, and thus the focus on their consequences and users of the Internet and technology in general is not unique to China. What distinguish the appraisals of the Internet (and its users) in the series, I think, are the inseparable ties between prudential and moral consideration. In other words, the goodness of the Internet can only be guaranteed if the Internet users are virtuous in the broadest sense.

\subsection{China's Internet and Confucianism: A Walzerian Perspective}

I have shown that the CCP evaluates the Internet and its impacts on the basis of Confucian values. Particularly, it is based on Internet's actual and potential contributions to the common good and its impacts on people's development into virtuous beings. While the CCP's account of "the common good" and "virtuous being" in relation to the Internet is justified or not is not unquestionable, acknowledging the claim that China's Internet is developed (and implemented) with Confucian values has a significant implication for intercultural normative analysis of digital media. For instance, international observers and critics often attack the CCP's Internet policy on the basis of liberal values. If China's Internet is designed and built on a set of values (i.e. Confucian values) that is distinct from, and sometimes incompatible to, liberal values, and this set of values is a legitimate option in itself, then the liberalist critique of the Chinese government ought to be reconsidered. Particularly, if the Internet embodies (liberal) values that cannot be readily accommodated by a Confucian outlook, it seems that the Chinese government's Internet policy is, at least, justified from a Confucian perspective. ${ }^{20}$ In

\footnotetext{
20 If such a consideration is legitimate, it will lead to a series of controversial questions pertaining to China's Internet policy (in the International context), especially the issues related to censorship and human rights in China, because it appears to lend support to the Chinese government's Internet policy. It is not my intention to argue in favour of censorship here, and my discussion in itself does not support such a conclusion. Such a
} 
a similar vein, the Chinese government has drawn from Confucian values and tried hard to promote the idea of harmonious Internet. However, realising the idea of harmonious Internet, derived from Confucian values, requires the Internet and the values it embodies to be compatible with Confucian values. Both issues invite us to rethink if the Internet is indeed compatible with Confucian values.

While there is abundant research on the embedded values in the Internet, there is surprisingly little discussion on the compatibility of the Internet and Confucianism. Mary Bockover's (2003) article, entitled "Confucian Values and the Internet: A Potential Conflict", appears to be the most direct attempt to address this issue. However, it has been some years since the publication of this article, and there have been novel developments of the Internet, e.g. Web 2.0, and, with new philosophical and ethical discussions have taken place, it is an appropriate time now to re-examine the issue.

Bockover argues that the Internet promulgates values that threaten Confucian values. In other words, she claims that the Internet is incompatible with Confucianism. Although, in a qualified sense, I agree with Bockover's conclusion, I think her argument is mistaken. In the following, I will revisit her argument and show why it has failed. I will then offer an alternative argument to show why the Internet in its present state is indeed largely incompatible with Confucian values.

\subsubsection{Bockover on Confucian Values and the Internet}

Before proceeding to my analysis, however, it should be stated explicitly that both Bockover's and my argument against the compatibility of Confucian outlook and the Internet reject the impact view of technology. Since the impact view of technology assumes technology itself to be neutral, according to this view, the Internet is, by definition, compatible with all value systems. In this respect, any arguments for or against the (in)compatibility of the Internet and Confucian values must presuppose a non-neutral view of technology. I shall not provide additional arguments for a value-laden view of technology here, suffice it to say that it is wellsupported by recent philosophical, social and empirical studies of technologyparticularly in the field of science and technology studies (e.g. Winner 1986; Brey 2010a).

conclusion can only be drawn if one has ostensibly demonstrated Confucianism does support censorship, which is not my objective here. 
Bockover begins her argument by asserting that the Internet is driven by "the Western values of free expression, equality, and free trade" which are in conflict, "perhaps even dramatically", with Confucian values (Bockover 2003, p. 160). In another place, she states that the Internet "is currently the most effective form of communication available to promote the first-world value of [personal and political] autonomy" that "stands in sharp contrast to the traditional Confucian system of values [...]" (Bockover 2003, p. 163). In other words, Bockover's argument can be formulated in the following:

(B1) The Internet embodies the Western values of free expression, equality and free trade or the idea of personal and political autonomy.

(B2) Western values of free expression, equality and free trade as well as the idea of personal and political autonomy are incompatible with Confucian values.

(BC) Therefore, Confucian values are incompatible with the Internet. ${ }^{21}$

In order to evaluate Bockover's argument, therefore, it is necessary to examine (i) whether Confucianism necessarily fails to accommodate those "Western" values and, thus, are incompatible with those values, i.e. (B2), and (ii) whether the Internet at its present state actually embodies those values, i.e. (B1).

Accordingly, Bockover's argument can be viewed as another instance of the long and continuing debate on whether Confucianism is compatible with Western ideals of human rights, e.g. freedom of expression, equality of opportunity, and (capitalist) socioeconomic growth such as free trade. ${ }^{22}$ So construed, the argument is

\footnotetext{
21 Unfortunately, it is relatively unclear in Bockover (2003) how, and in what sense, the values of free expression, equality and free trade or the value of personal and political autonomy are the embedded values of the Internet except through a number of anecdotes. The most explicit elaboration of the embedded values of the Internet is:

"Internet activity now embodies and perpetuates a view of freedom that has nothing to do with [what is right, true and fair, what is proper and in due measure]. [... ] The Internet does not lend itself to... self-regulation, and so the concept of freedom that it now expresses is almost exclusively defined by the desire for economic freedom (to get rich) and personal freedom (to have an equal opportunity to do and say what one wants)" (Bockover 2003, p. 170).

22 It should be noted that Bockover has assigned a very narrow notion of freedom to the Internet, e.g. unrestricted freedom of expression, based on the assumption that the Internet is not and cannot be (self-)regulated. However, such an understanding of freedom (of expression) appears to turn her argument into a straw man, as the Internet
} 
ostensibly weak. It has already been pointed out by a number of philosophers that Confucianism is compatible with human rights and (capitalist) socioeconomic growth. ${ }^{23}$ As May Sim succinctly states " $[\mathrm{t}]$ here is a way that Confucians can make sense of rights out of the resources of their own tradition" (Sim 2004, p. 338). Yet, the point here is not that there is a definitive answer to the compatibility between Confucianism and values such as freedom of expression, equality of opportunity and/or the idea of free trade, but rather to point out that the relation between Confucianism and the values mentioned by Bockover is less contentious than she has construed. Hence, there is sufficient reason to question the truth of (B2).

Perhaps, then, there is something more to Bockover's argument against the compatibility of Confucian values and the Internet. Here, it is possible to take a hint from her emphasis on personal and political autonomy as the embedded value of the Internet. Bockover argued that the idea of personal and political autonomy embedded in the Internet is derived from a notion of autonomous and rational self (Bockover 2003, pp. 165-170). I contend that if the Internet has embodied the idea of personal and political autonomy derived from a notion of an autonomous and rational self, then Confucianism will have enormous difficulty accommodating the Internet since Confucianism lacks a notion of personal and political autonomy (see, e.g. Chan 2002). Moreover, Confucianism presupposes a relational self that runs against the notion of the self merely as an autonomous and rational being. ${ }^{24}$

Of course, this line of argument depends on whether the Internet has truly embodied the idea of personal and political autonomy derived from an autonomous and rational self. Unfortunately, Bockover has not explained how-if it is in fact so- the Internet embodies the idea of personal and political autonomy and the specific notion of the self. However, her description of the Internet as a space without (self-)regulation, which fosters a "concept of freedom [...] exclusively defined by the desire for economic freedom (to get rich) and personal freedom (to have an equal opportunity to do and say what one wants)" (Bockover 2003, p. 170), fits nicely with a view of the Internet envisioned by cyber-libertarians. As Langdon Winner (1997) characterised, cyber-libertarianism consists of (i) an unquestioned

will be incompatible to most —if not all—coherent value systems, including a liberal value system.

23 For discussions of the compatibility of Confucianism and human rights, see, e.g. Chan (2002), Sim (2004) and D. Wong (2004b). And, for discussions of the compatibility between Confucianism and (Western) capitalism, see Nuyen (1999).

24 See, Chapter 4, section 4.2.3. 
belief in technological determinism of adapt or perish, (ii) a radical individualism, especially for pursuit of rational self-interest such as wealth, power and sensual pleasure, and (iii) a supply-side, free-market capitalism (and, all together, they usher (iv) a Jeffersonian vision of citizen and political society) (see also, Bell et al. 2004, pp. 43-46). Most relevant to the present discussion is the idea that the Internet has enabled (and, simultaneously, rested on) a radical individualism, which, in turn, has embodied and fostered the idea of personal and political autonomy.

Granted, whether the Internet (and, in general, digital media) - even in its early stage - truly accords to the view that cyber-libertarianism is debatable, it is hard to deny that if it is indeed so, the Internet and Confucian values cannot be compatible, as the Internet is then rested on a radical individualism that promulgates a radical version of personal and political autonomy, which essentially comes into conflict with the relational self presupposed by Confucianism. So construed, Bockover's argument will be successful.

Yet, as I have already illustrated in Chapter Three, there is a profound development of the Internet since early 2000s generally known as Web 2.0 (O'Reilly 2005a, 2005b). Although the definition of Web 2.0 remains elusive, its association with openness, collaborative and participatory nature of the Internet contrasts greatly with the closeness and solitary nature of Web 1.0. ${ }^{25}$ And, the cyber-libertarian view of the Internet, which Bockover's argument relies on, is primarily affiliated with Web 1.0, i.e. it embodies radical individualism by enabling people to publish webpages that are under their full control (because of the closeness of webpages) as well as to retrieve and consume information via search engines self-sufficiently and without the need of interaction. I am not, of course, suggesting Web 2.0 is irreconcilable with radical individualism and/or cyberlibertarianism (see, e.g. Song 2010, Dahlberg 2010). Still, the shift towards Web 2.0, I think, has transformed the nature of the Internet and the values it embodies. Particularly, Web 2.0, with its built-in open, collaborative and participatory nature, has instilled the Internet with a (new) dimension of sociality that, ideally, encourages community building and cooperation (Fuchs et al. 2010, Fuchs 2010). A look at the Web 2.0 applications such as wikis, folksonomies, mashups social networking sites, etc. should illustrate that sociality is now being designed and coded into them, which thus clearly distinguishes them from earlier Web 1.0 applications (Beer \& Burrows 2007, Papacharissi \& Gibson 2011). For instance,

25 See, Chapter 3, section 3.1. 
social networking sites, which often viewed as the web 2.0 application par excellence, are explicitly aimed at (re)creating online people's social connections and relationships (boyd \& Ellison 2008; boyd 2010a). In this respect, the design and use of Web 2.0 applications does not-at least, not primarily-have in mind the idea of persons isolated from each other; instead, they fully recognise the primacy and significance of social and relational dimension of the people (see, e.g. Albrechtslund 2008, Mallan \& Giardina 2009, boyd 2010a).

The shift from Web 1.0 to Web 2.0 is also associated with a change in our sense of the self (Song 2010, Ess 2010, Papacharissi \& Gibson 2011, Bakardjieva \& Gaden 2011). On a more optimistic note, Charles Ess argues that the development of digital media, especially Web 2.0 and social media, has de-emphasised individual privacy required to sustain (radical) individualism and, thereby, has ushered a (re)turn to a (more) relational understanding of the self (Ess 2010, pp. 110-112). ${ }^{26}$ Besides, if decisions and actions in a personal realm are representative examples of autonomous choices and actions, then we can also observe a turn to a (more) relational understanding of autonomy in and through Web 2.0 and social media. For example, the design and use of social media such as Facebook, Twitter and, lately, Google+ explicitly aims to harness collective decisions and facilitate collective actions. And, by foregrounding the decisions made and actions done by people in the individual's social connections and relations, e.g. aggregation of "Like", "Retweet" and " +1 ", social media has made people more open to influence from their social connections and relations in their decisions and actions. Hence, I think, it is fair to claim that the design and use of social media is based, not on an autonomous view of decision and action, but on a relational view. In short, I think it is safe to conclude that this new version of the Internet, i.e. Web 2.0, is based on a different sense of the self and idea of autonomy.

Yet, if it is indeed true that Web 2.0 has introduced a (new) dimension of sociality and relationality to the Internet and (re)turned us to a (more) relational understanding of the self, then Bockover's argument becomes obsolete because the argument invokes a different view of the Internet, which sees it as an embodiment of radical individualism and promotes the idea of (radical) personal and political autonomy. In short, Bockover's argument fails because her view of the Internet in

26 At the same time, Ess also argues that there is a change in the sense of the self in East Asian cultures from a relational self to a (more) individualistic understanding of the self. In short, Ess is arguing for a hybridisation of different senses of the self but not a change in singular direction, i.e. from the individualistic self to the relational self and vice versa. 
the argument no longer accords to the Internet at its present state; therefore, (B1) is false. In effect, if Ess's argument is right that Web 2.0 and social media (re)turn us to a (more) relational understanding of the self, the Internet will be more hospitable to Confucianism. The question, then, is whether Confucianism is compatible with the latest development of the Internet, i.e. Web 2.0?

\subsubsection{Confucian Social Media: An Oxymoron?}

The question, however, resists an immediate answer, for the term 'Web 2.0' is itself vague and ambiguous and there is a plethora list of diverse web applications associated with Web 2.0. Hence, the term 'Web 2.0' in the question must be qualified if it is to be meaningful at all. I shall limit my analysis to social media, as they are most characteristic of the social and relational dimension of Web 2.0; and, therefore, they are also the most suitable candidates to test the (in)compatibility of Web 2.0 and Confucian values. As Ess (2010) has anticipated, with the increasing prominence of Web 2.0 and social media, there will be a hybridisation of individualistic self and relational self. Here, it is possible to question whether a (more) individualistic but still primarily relational self that Web 2.0 brings about can be coherently conceived from a Confucian outlook. However, I shall not attempt to answer this question because it goes beyond the scope of the (in)compatibility of social media and Confucianism and runs into the foundation of Confucian philosophy. Instead, I will only examine whether social media as it is now being designed (and used) is compatible with Confucian values.

\subsubsection{Affordances and Dynamics of Social Media}

My analysis is built on danah boyd's (2010a) theoretical and ethnographic studies of social media (see also, boyd \& Ellison 2008). ${ }^{27}$ In her works, boyd has examined the architecture of social media as well as the affordances and (communicative) dynamics supported by it. As she rightly points out, although social media does not determine users' behaviours and practices, its architecture, through its affordances (i.e. by opening up and/or making more accessible some possibilities of decision and action and vice versa) and dynamics, has shaped users' behaviours and practices. So

\footnotetext{
27 boyd's (2010a) article only focuses on social networking sites, i.e. a type of social media; but her analysis of social networking sites is based on the notion of networked publics, which can also be generalised and applied to social media.
} 
understood, my analysis is about whether social media, because of its design and use, tends to be considered as (un)desirable from a Confucian point of view.

boyd seeks to understand social media through the notion of networked publics, which are publics "transformed by networked media [e.g. information and communication technologies], its properties and its potentials" (boyd 2010a, p. 42). Networked publics, accordingly, distinguish themselves by their structural foundation, i.e. bits, and the affordances available to the architecture of bits, namely "persistence", "replicablity", "scalability", "searchability" and "shareability"28 (boyd 2010a, pp. 40-42, 45-48; Papacharissi \& Gibson 2011, p. 76). boyd argues these properties of networked publics have supported three dynamics, which have come to dominate the network publics, namely

Invisible audiences: Not all audiences are visible when a person is contributing online, nor are they necessarily co-present.

Collapsed contexts: The lack of spatial, social, and temporal boundaries makes it difficult to maintain distinct social contexts.

The blurring of public and private: Without control over context, public and private become meaningless binaries, are scaled in new ways, and are difficult to maintain as distinct. (boyd 2010a, p.49)

It is these dynamics, as I shall argue, that sit uncomfortably with Confucian values. However, in order to show why social media will be seen as undesirable from a Confucian point of view, I shall now briefly return to Confucian ethics.

\subsubsection{The Confucian Way of Life Revisited}

In Chapter Four, I have pointed out that familial relationships and social roles have special significance to Confucian ethics. Confucians believe that people's roles are constitutive of their personhood, i.e. whether someone is truly and/or fully a person depends on his performance of the roles. ${ }^{29}$ And, to properly perform a role is to fulfil the responsibility prescribed by that role. People's roles and their performance

\footnotetext{
28 boyd (2010a) does not include "shareability" as one of networked publics' affordances. But, as Papacharissi and Gibson rightly note, enacting on the properties of networked publics essentially prompts sharing. (Papacharissi \& Gibson 2011, p. 76)

29 I am not, however, arguing that in Confucianism, a person is exhausted by his roles. To hold this view, as KIM (2011) convincingly argues, is to deprive Confucian persons of (moral) agency.
} 
of them, therefore, are central in the Confucian way of life. This implies that Confucians will scrutinise carefully the relations between technology and (social) roles or, more precisely, they will examine if and how technology influences and transforms the nature of specific (social) roles and the responsibility prescribed by them. ${ }^{30}$ This exercise should not be viewed merely as historical or sociological, i.e. to identify what (social) roles have changed in relation to technology, because the moral significance of roles and a person's performance of them is too normative. Moreover, technology can enhance or deter people in fulfilling of their roles. For Confucians, therefore, whether social media is desirable or not depends first and foremost on its impacts on (sole) roles.

However, not all roles are equal to Confucians. Confucianism prioritises familial relationships over other relationships. As many commentators of Confucianism have noted, central to the Confucian way of life is the virtue of filiality and fraternity (Xiaodi 孝悌) and the fulfilment of filial and fraternal responsibility (see, e.g. D. Wong 2008; FAN 2010, Chapter 1-3; KIM 2010). For instance, it is stated in The Analects 1.2, "[...] filial piety and respect for elders constitute the root of Goodness ["ren"]" (Slingerland 2003, p.1). Familial relationships are of utmost importance to Confucians because they believe that these relationships are the prototypical natural affectionate relationships (i.e. parent-children, siblings) through which they allow one to learn to be, and eventually to become, a true and full person. As I have pointed out, Confucians regard all non-familial relationships to be the extension of familial relationships; assuming familial roles and fulfilling their role responsibility are viewed as a moral preparation for people to perform non-familial roles. In the Confucian way of life, therefore, familial roles and the responsibility prescribed by them are more important than non-familial roles.

Finally, the importance of rites $(L I)$ cannot be neglected in the Confucian way of life, as one's roles should not be performed arbitrarily-a person ought to perform his role and to fulfil the role responsibility appropriately. Rites provide thick instructions, not just thin principles, as to what counts as appropriate in various contexts. Here, it is useful to note that rites do not only limit to formal rituals, but also include minute rituals for everyday practices (FAN 2010, pp. 171-

\footnotetext{
30 To be sure, the significance of professional roles has already been studied in engineering ethics and other professional ethics, but the notion of (social) role in Confucian ethics should be conceived as much broader.
} 
172). Since the appropriateness of decisions and actions is determined by rites, following rites is essential to the Confucian way of life. To live a Confucian way of life means that one is to ceaselessly perform his role and fulfil the role responsibility appropriately through following rites. Of course, it is not to assert that Confucians follow rites unreflectively and without exceptions, as it entails Confucianism to have no room for (moral) agency and changes in rites, and thereby renders Confucianism to absurdity. As many have pointed out, Confucianism does have room for (reflective) disregards and exceptions for rites (Y. LIU 2004; KIM 2009; FAN 2010, pp. 181-188). Yet, rites cannot be abandoned ad hoc, it is allowed only when and insofar as doing so coheres with other Confucian values. In short, rites cannot be separated or withdrawn from the Confucian way of life; as $\operatorname{KIM}(2009,2010)$ has rightly noted, the Confucian way of life differs from others in that it has incorporated rites in its self-cultivation and self-transformation.

\subsubsection{The Confucian Way of Life and Social Media: A Mismatch?}

I have highlighted three aspects of the Confucian way of life, namely (i) roles and role responsibility, (ii) filiality and fraternity (Xiaodi) and (iii) rites $(L I)$. These aspects are particularly relevant to the present discussion of the (in)compatibility between social media and Confucian values. In the following, I illustrate why and how social media, with the dynamics identified by boyd (2010a), is a poor match with the Confucian way of life.

The existence of invisible audiences on social media is problematic to the Confucian way of life. Since users' audiences can be neither visible nor co-present at the time when the users 'say' (or 'do') something on social media, e.g. social networking sites, microblogs, etc, they are in effect interacting with someone who they do not know with certainty. This is not to claim that invisible audiences do not exist prior to social media. Invisible audiences-who are unknown and/or absentexist in the offline world too. In the offline world, people can interact with someone who is not co-present through writing. There, however, the person absent is not unknown. Similarly, people can interact with someone who they do not know through writing and/or (public) speech, but, they can still draw a sensible boundary of intended and unintended audiences through the writing's and speech's style, genre and context, and thereby assume and perform their roles accordingly. In this respect, (invisible) audiences in the offline world are to a large degree identifiable to people. Social media, on the other hand, admits a much lesser degree of identifiability. This lack of identifiability is best illustrated by social networking 
sites: when users disclose themselves through social networking sites such as Facebook, in which other people-as long as they have been granted permission (i.e. in Facebook's default setting, when they are a 'friend') - can view them, these people are all audiences, and they are indifferent to the users too. ${ }^{31}$ The users, in this case, do not specify their audiences and differentiate them by default. This uncertainty about their audiences makes it difficult, if not impossible, for the users to assume and perform the correct roles with respect to their (online) audiences because in order to do so, the users need to know who they are.

It becomes especially troubling to Confucians if we consider the online world to be a continuum of the offline world, as all users' offline relationships, e.g. family, friends, colleagues, etc., are at the same time potential invisible audiences so long as they are online too; being (potential) invisible audiences, however, they have barred the users from assuming and performing the right roles with regard to them even if the users have no problem doing so in the offline world. So understood, social media at its worst engenders a world in which people constantly fail to assume their roles. Since (social) roles are, according to Confucianism, constitutive of one's personhood, and one can only become a person if his roles are properly assumed and performed (with the role responsibility properly fulfilled), Confucians should evaluate social media negatively because of the (online) world it engenders.

Invisible audiences, of course, are not only a problem for the Confucian way of life but also a problem for any users of social media, as they too need to know if what they say or do is appropriate or not. To counter the problem that arises from invisible audiences, one strategy for the users is to imagine who they are interacting with when they are using social media. This strategy helps to reduce uncertainty on the users' side because the users can then delimit their behaviours and practices in accordance to the type of audiences imagined (boyd 2010a, p. 50; Paracharissi \& Gibson 2011, p. 80-81). Yet, I think imagined audiences are too thin for the users to determine their roles correctly. Since imagining audiences is essentially a strategy for limiting behaviours and practices, unless the users have imagined a concrete

\footnotetext{
${ }^{31}$ It is important to bear in mind that 'saying' (or 'doing') something on social media is not limited to self-disclosure, e.g. managing your pages and content, etc.; it also includes writing and commenting on other's pages as well as forwarding their content. In the latter cases, although the users are interacting with and/or related to some identified audiences, and so they can assume their roles accordingly, uncertainty remains insofar as their audiences' pages can be viewed by others. In those cases, the unidentified others become the users' invisible audiences.
} 
relationship, it cannot tell what roles they ought to assume and perform. At the same time, because of the existence of invisible audiences, even if the users have assumed and performed some roles correctly through some types of imagined audiences, they remain-by the default setting of social media-constantly open to other relationships they are unaware of, and thereby, cannot account for. In short, the existence of invisible audiences on social media has created an environment which renders the Confucian way of life hard to live by.

The same is also true of following rites, i.e. another important component for living the Confucian way of life. ${ }^{32}$ To reiterate, rites are a set of proper conducts and attitudes for a specific situation. The multiplicity and simultaneity of relationships that social media affords make it difficult —again, if not impossible - to follow rites, as which rites to follow are determined by who the person is interacting with. This issue is further aggravated by another dynamic of social media, namely the collapsed contexts. With social media, contexts are mixed and merged by their default setting; however, people need to know what contexts they are in if they are to know what are the proper conducts and attitudes to have. In this sense, contexts are ethically constitutive of the Confucian way of life, as they require people to have a proper set of conducts and attitudes which are context-dependent, e.g. a familial context and a professional context clearly demand a different set of proper conducts and attitudes. ${ }^{33}$ In other words, the Confucian way of life needs to maintain, at least, an epistemic separation of various types of contexts. Collapsed contexts on social media, therefore, entail an enormous difficulty for people to know what are the proper conducts and attitudes to have in the (online) world. ${ }^{34}$

\footnotetext{
32 It should be noted that I am not arguing for the difficulty or impossibility of following rites, i.e. to have the set of proper conducts and attitudes, on the ground of the texture and experience of the online world being too impoverished to be qualified as really following rites. I think this is a legitimate consideration for those who examine the compatibility between social media and the Confucian way of life; this line of argument, however, will depend on empirical investigations of the texture and experience of the online world. And, it is also not unreasonable to speculate-with technological advancement-the texture and experience of the online world will only become richer. 33 I am, of course, not suggesting that there is only a single set of proper conducts and attitudes for each and every context, or that a context exhaustively determines what qualifies as proper conducts and attitudes.

34 Also, the collapsed contexts do not only appear on social media, as Turkle described, "demarcations of [contexts] blur as technology accompanies us everywhere, all the time. We are too quick to celebrate the continual presence of a technology that knows no respect for traditional and helpful lines in the sand." (Turkle 2011, pp. 161-162)
} 
Finally, the blurring of public and private should worry Confucians too. However, unlike the current debate on this issue, which is often framed as a privacy issue (see, e.g. Barnes 2006; boyd 2010a, pp.51-52; Light \& McGrath 2010; Hull et al. 2011; Papacharissi \& Gibson 2011), Confucians' worry on the blurring of public and private is of a different nature. Firstly, Confucians do not distinguish sharply between the public and private with respect to self-cultivation and selftransformation because they believe people's self-cultivation and selftransformation in the private sphere will essentially carry into their public sphere (and vice versa). Hence, both the public and private are of equal moral significance as they are, and should be, subjected to the same level of (moral) scrutiny. Secondly, in accordance to Confucian non-individualistic views of person, the term 'private' is not to be understood at an individual level; instead, it is to be understood at a familial level. Hence, the private sphere refers to the familial sphere from a Confucian point of view. So construed, the Confucians' worry over the blurring of public and private is not about individual privacy but about changes at the familial level.

Confucians' insistence on the priority of familial relationships and the importance of filiality and fraternity, however, has already hinted that a separation between the public and private ought to be maintained. In Confucianism, the familial relationships are a model for other non-familial relationships. Family (or, the familial sphere) is believed to be distinct from other spheres in that the roles and role responsibility in familial relationships are driven by natural affections and trust, i.e., parent-children and sibling; therefore, it provides qualitatively different feedback to people in their learning to become a person. ${ }^{35}$ And, it is also where people learn to socialise, by assuming and performing the roles and fulfilling the role responsibility, eventually achieving proper conducts and attitudes towards non-familial members in the society. ${ }^{36}$ Hence, family is essential in people's (moral) development from the Confucian point of view.

\footnotetext{
35 This is, of course, not to assert family (and familial relationships) in reality will always be affectionate. As KIM (2010) rightly points out, Confucians do admit the fact that there are unaffectionate-perhaps, even pernicious-family and familial relationships. Hence, the notion of affectionate family and familial in Confucianism should be taken as a normative ideal and not a descriptive claim.

${ }^{36}$ See KIM (2010) for an illuminating account of how family and familial relationships can serve as a model of "relational strangership".
} 
The blurring of public and private leads to the disappearance not only of the private sphere but also the familial sphere; in doing so, it also takes away the space where people learn to become a person and achieve proper conducts and attitudes towards non-familial members. Indeed, by breaking down the barriers between the public, private and familial sphere, it seems to neutralise familial and non-familial relationships, as well as depreciate the importance of the former. Most importantly, perhaps, is that without the familial sphere, every (wrong)doings are subjected to risks of public shaming, which is detrimental to people's development. In short, the blurring of public and private has eliminated a domain crucial to the Confucian way of life.

To summarise, the three dynamics supported by social media, i.e. (i) invisible audiences, (ii) collapsed contexts and (iii) the blurring of public and private, have engendered an online world that is rather inhospitable to the Confucian way of life. Alternatively, since the Confucian way of life is hard to live by with social media, I believe Confucians will inevitably see it as undesirable.

\subsubsection{Recontextualising the Confucian Way of Life, Redesigning Social Media}

So far, I have illustrated that the dynamics supported by social media are not conducive - and, perhaps, even detrimental - to the Confucian way of life. Hence, even if social media (and Web 2.0) has (re)turned us to a (more) relational understanding of the self, it remains difficult to be accommodated by a Confucian outlook due to its architecture. However, I do not mean to suggest that social media cannot be beneficial or that every use of social media is necessarily harmful to people, especially to those who sought to live the Confucian way of life. As a matter of fact, it is hard to deny the benefits brought by social media, e.g. parents and children get to know each other better through sharing content, people can (re)connect to friends who would otherwise be forgotten, etc. In this respect, it seems absurd for Confucians to deny the use of social media in entirety.

Confucians, of course, can maintain a pragmatic attitude towards social media, i.e. it is acceptable insofar as social media does contribute to the realisation of some Confucian values. It is also useful to highlight the fact there is a variety of social media designed for and used with different purposes (boyd \& Ellison 2008). Some of them may well be able to help realise the Confucian way of life, even if it is only partially so. More importantly, it should be reminded that although social media does shape people's behaviours and practices through its architecture and design, it does not determine people's behaviours and practices. In other words, people can 
still develop behaviours and practices desired and demanded by the Confucian way of life. In light of this, I fully agree with Ess's (2010) and Vallor's (2010) suggestion of returning to virtues, which enables people to live well with social media. In a similar vein, I believe Confucian values and the Confucian way of life can be recontextualised to offer valuable advice and direction for incorporating social media into our life.

I shall not pretend to have a fully working account of how Confucian values and the Confucian way of life can be recontextualised to face the challenges from social media. Instead, I will only outline three preliminary responses to the three dynamics supported by social media from a Confucian perspective:

1. A skilful engagement of social media. For Confucians, the main challenge from invisible audiences is to know who one is interacting with, and, thereby, to assume and perform the role accordingly. While it is true that social media is geared towards publicity by default, in many social media it is not impossible to override the default setting and achieve a more expected range of audiences. There are also other strategies the users can adopt to demarcate different groups of (online) audiences, e.g. social steganography (boyd 2010b). Interestingly, Paracharissi and Gibson (2011) call these skills "an advance form of digital literacy" for protection of one's privacy; however, it is equally useful to the Confucian way of life, which requires one to know who they are interacting with. At the same time, one can also imagine the Confucians will require the users to be more thoughtful about their connections on social media in order to limit the (invisible) audiences. From this perspective genuine relationships go beyond simple connections.

2. Reinvigoration of rites in the online world. The same is true to the challenge from collapsed contexts, too. Although the contexts are often mixed and merged by default, it is not impossible to demarcate different contexts by overriding the default setting. This, again, requires the users to have the digital literacy of (online) context management. On the other hand, there is the need for Confucians to reconceptualise the online world and to reconsider if the mixed and merged contexts warrant a new form of rites for the online world. Rites, as I have pointed out, are not fixed and unchangeable; since the ultimate objective of rites is to ensure the realisation of Confucian values, e.g. benevolence (Ren) and harmony $(\mathrm{He})$. It is not unreasonable to reappropriate rites creatively in and for the online world. 
3. Prioritisation of the offline world. Finally, I think the blurring of public and private has posed the most serious challenge to the Confucian way of life. Although it might be true that with the right setting and adequate skills of online context management, it is possible to recreate the familial sphere on social media. However, the familial relationships are still essentially to be on par with other non-familial relationships on social media, as there is no way to prioritise the former over the latter at the moment. In light of this, I believe Confucians will have to resort to affirming the priority of the offline world over the online world. Accordingly, social media can only be viewed as a supplement of the offline world.

While I have provided a sketch of the response to the challenges from social media, it may remain difficult to see how the Confucian way of life, as I have illustrated, can positively endorse social media. After all, the challenges arise from the architecture and design of social media, and the response I have provided risks "overstating the role of human agency" because of its lone emphasis on people, i.e. users (Light \& McGrath 2010, p. 305). I think this concern is well-founded. Yet, if the challenges of social media arise from its architecture and design, then it should also be possible to redesign it in such a way that offer us new dynamics and reduce the prominence of certain dynamics. Before ending this section, I want to venture into two possible designs that could make social media more hospitable to the Confucian way of life:

1. Designing contextual awareness into social media. In their discussion of privacy on Facebook, Hull et al. (2011) noted that Facebook has created "contextual gaps" to the users and, thereby, leads to various privacy issues. They argued that by making the flow of information more transparent to the users, the contextual gaps can be mitigated. Then, they offered several design suggestions, the most interesting suggestion being "from the point of view of the reader: attached to each update could be a "view all of Mary's updates" option, which would subtly remind users that the same option applies to their own updates. It could even be designed to send a notice to users: "Mary has just looked at all of your updates." [...] [In this way,] users would increasingly view their Facebook identities as subject to constant surveillance, and modify them accordingly. If I knew that Mary always looked at all my updates, I might update with her in mind" (Hull et al. 2011, p. 299). For the purpose of the Confucian way of life, 
the design from a reader perspective appears to highlight who the users are (or will be) interacting with in contrast to a broadcaster perspective in which the audiences is left implicit in the design.

2. (Re)introduction of role responsibility into social media. Another design suggestion is to assign responsibilities to other users in the form of identity and content management (e.g. personal profiles and/or shared content). By assigning different levels of responsibility to different groups of users, the priority of relationships can be (re)introduced into social media, and relatedly, the familial sphere can also be (re)created in the online world. In accordance to the Confucian way of life, the familial members will have a high(er) level of responsibility (and, therefore, permission) to manage the user's profile and content shared, which can either take the form of suspension, modification or even screening of information and content shared by the user. In this way, the familial members can actively assume and perform their roles in the online world. At the same time, it also offers an enclosed space in which the users can learn from their familial members. It is likely that this design suggestion will inevitably invite criticism of breaching the user's privacy; however, such a criticism will only be valid if one has an individualistic understanding of privacy (and the private sphere), which is rejected by Confucians. In effect, this design suggestion appears to be morally desirable from the Confucian perspective, as it truly allows the users of social media to fulfil their responsibility prescribed by their roles.

Unfortunately, as social media continues to evolve and user's behaviours and practices are (co-)shaped by social media, there is no guarantee that either the response or the design suggestions outlined can resolve the tension between the Confucian way of life and social media (and, in general, Web 2.0). Yet, precisely because the technology continues to evolve and user's behaviours and practices are (co-)shaped by it, a sustained inquiry of their relation is indispensable.

\subsection{Conclusion}

To reiterate the objective of this chapter, my aim is to offer a case study of applying the Walzerian approach at a global level. In other words, I seek to demonstrate the importance of other notion of the self (i.e. the Confucian self) and views of the good life (i.e. the Confucian view of the good life) in normative analysis of digital media 
and the good life, especially in an intercultural context. In the Section 5.2 and Section 5.3, I have demonstrated how the CCP's position towards the Internet is by and large based on Confucian values: in the various discourses on the benefits and harms of the Internet as well as the problems related to it, it is possible to detect references - either implicit or explicit-of Confucian values. If my interpretation is correct, there are significant ramifications on the study of China's Internet. Particularly, it calls for a serious (re)consideration of the often liberalist critique of China's Internet. Indeed, if the Internet and the values it embodies are incompatible with Confucian values and Confucianism is a legitimate value system, it seems that restrictions of the Internet in accordance with Confucian values can be justified. ${ }^{37}$

In Section 5.4, I examine whether the Internet is incompatible with Confucian values. I begin by revisiting Bockover's (2003) article, which directly addresses this issue and argues for the incompatibility of the Internet and Confucian values. Although a specific interpretation of Bockover might be plausible, her argument is flawed by an outdated view of the Internet. I note that the Internet has moved from Web 1.0 to Web 2.0, which (re)turns us to a (more) relational understanding of the self. However, using social media as an example, I argue that, despite the shift in the understanding of the self, due to its architecture, social media is also inhospitable to the Confucian way of life. If we put aside the view that the Chinese government only uses Confucianism as a smoke screen to achieve its malevolent objectives, the incompatibility between social media (and, in general, Web 2.0) can offer us a valuable explanation for its deep-seated uncomfortability with social media.

Yet, if social media is indeed inhospitable to the Confucian way of life, then how should the Confucian way of life proceed? In other words, as long as social media is here to stay, is the Confucian way of life impractical and/or inadequate? At the end of this chapter, I have offered some reflections on the Confucian way of life as well as the design of social media in hopes of reconciling them.

\footnotetext{
37 To emphasise once again, I am not claiming that the censorship of the Internet is -or, even can be-justified. I am only claiming that the critique of China's Internet policy should be based on Confucian values. In fact, there are a number of attempts to show that freedom of speech is just as important for Confucianism. See, e.g. BAI (2008) and D. Wong (2004b).
} 



\section{CHAPTER SIX}

\section{Digital Media, Recommendation and Design On Being a 'Paternalistic' Philosopher}

In reflecting on the current state and future prospects of philosophy and ethics of technology, Philip Brey (2010b) and Peter-Paul Verbeek (2010) have noted that the field in its current state, especially in the discussions of normative issues surrounding technology, has focused too narrowly on moral values, i.e. the right and the just. The main question in the field can thus be summarised as: Is this or that technology morally (or politically) dubious? And, if so, what should we do about it? As such, philosophers have spent most of their energy establishing boundaries between technology and humanity and to prevent intrusions of technologies into certain areas of people's life. Accordingly, philosophers and ethicists conceived of themselves as protectors of humanity from threats of technology. In this respect, the situation in ICE is not too different from philosophy and ethics of technology. Most researchers in the field of ICE have, by and large, ignored the issues that fall outside the scope of the right and the just.

For Brey and Verbeek, this narrow focus is unpromising, and they argue that philosophers should broaden their scope of inquiry to cover the issues related to people's good life because of the possible impacts of technology on people's ways of living. Philosophers should, in other words, put more effort into investigating the potential contributions of technology to the good life. I think Brey and Verbeek have rightly directed our attention to an important but neglected aspect. As I have tried to show in the previous chapters, digital media and digitally-mediated 
practices have raised various issues pertaining to the good life and have inspired numerous debates on whether and how we should live with them. In this sense, I agree with Brey and Verbeek that researchers should put more effort into improving human-technology relationships (and society-technology relationships) than merely to condemning technologies outright, but the question is: how should philosophers do that?

Verbeek's answer to this question lies in "an ethics of 'accompany technology' [that aims] to accompany the development, use and social embedding of technology" (Verbeek 2010, p. 52). This, Verbeek has argued, requires philosophers (i) to participate and intervene in the design and production of technology and (ii) to enable users and policy-makers to better understand technologies and their potential impacts on their good lives. While Verbeek and others have devoted considerable amount of works to the first half of this project through the ethics of and in design, relatively little effort has been directed at the second half of the project. ${ }^{1}$ This chapter continues with the discussion they have initiated, and my emphasis is on the second part of Verbeek's project. Here, I reflect on the role of philosophers with respect to digital media, argue for a specific role for philosophers, and illustrate how this role can be best fulfilled.

Researchers in the field of ICE have talked to each other about moral issues concerning technology, but few of them have talked about issues of technology and the good life, and even fewer have talked about technology and the good life with the public in the form of recommendation. Recommendations on digital media and the good life are often left to technologists and gurus. ${ }^{2}$ Many of them are, of course, capable experts on digital media. Yet, it is less clear that they are also experts of the good life. Given the potential benefits of informing the public of the impacts of digital media on the good life, the absence of philosophers is a curious state of affairs. And, I think, their absence in offering recommendations to the public is far from optimal. In the next section, I will examine why philosophers are seemingly reluctant to offer recommendations to the public. While there are many reasons, including sociological, conceptual and normative reasons, for philosophers to

\footnotetext{
1 Interestingly, Verbeek has mentioned that "the ethics of accompaniment also engages with the ways in which users help to incorporate technologies in their lives, and policy-makers help to shape the role of technologies in social practices." However, Verbeek has yet to pursue this part of "accompanying technology" in his own works. 2 See, for example, the prudential appraisals I have analysed in the Chapter 3 and Chapter 5 .
} 
refrain from offering recommendations, I shall focus on the normative reasons. More specifically, it appears that, according to a particular definition, offering recommendations can be viewed as paternalistic, and therefore it is prima facie wrong to do so. I provide an argument to show that the worry of paternalism is unfounded, because a form of paternalism engendered by technology is inevitable. Given the inevitability of paternalism, I note that philosophers should accept the role of offering recommendations to the public. I then briefly turn to design ethics, which has reconceptualised the role of philosophers and, in my mind, fitted well with the inevitability of paternalism. My brief discussion of design ethics, however, is not intended to offer arguments for or against design ethics. Instead, my objective is to point out that design ethics, while being one of the best responses to the inevitability of paternalism, it has to be supplemented by the practice of recommendation if it is to achieve its agenda. Finally, I argue that the Walzerian approach to digital media and the good life provides philosophers a useful starting point to take part in the practice of recommendation.

\subsection{Perils of Recommendation}

A number of reasons are proposed to account for why philosophers-and, scholars in general-have refrained from offering recommendations to the public. For example, Russell Jacoby (2000) has developed an interesting sociological account of the decline of public intellectuals. ${ }^{3}$ Steve Fuller (2004) has argued that there is a demise of the conceptual conditions necessary for the existence of public intellectuals in the 21st century. Likewise, Linda Martin Alcoff (2002) has discussed the doubts on academic integrity of the works directed to the public. Instead of pursuing them further, I want to focus on one aspect which, I think, has led philosophers to excuse themselves from the practice of recommendation, i.e. offering recommendations to the public can be viewed as paternalistic. It should be noted that not all recommendations to the public are paternalistic in a way that troubles philosophers and that whether recommendations are paternalistic is not independent from one's definition of paternalism. Hence, I first identify the relevant type of recommendations in question. I will then examine some characteristics of paternalism. Instead of arguing for a particular definition of paternalism, however, I will look at what makes paternalism objectionable, and

3 This study only discusses public intellectuals in the American context, but he has later attempted to revise and extend his thesis to other regions too, see Jacoby (2009). 
thus being prima facie undesirable. I argue that the type of recommendations in question can raise paternalistic worry because, like other paternalistic behaviours or practices, it seems to interfere with people's liberty and autonomy by imposing a view of the good life to them, and it also seems to insult people by telling people how that they should live their own lives and thereby debasing them.

Broadly speaking, a person $\mathrm{P}$ offers someone $\mathrm{Q}$ a recommendation when (i) $\mathrm{P}$ explicitly tells $\mathrm{Q}$ that $\mathrm{Q}$ ought to do $\mathrm{X}$ because (ii) ( $\mathrm{P}$ thinks that) doing $\mathrm{X}$ is good/good for S. ${ }^{4}$ Since my study aims at the relation between digital media and the good life, I will set aside the issues related to good simpliciter. As I will later show, the practice of recommendation becomes especially problematic when $\mathrm{S}$ refers to the person $Q .{ }^{5}$ For my current purpose, (ii) should be interpreted as: ( $P$ thinks that) doing $\mathrm{X}$ is prudentially good for $\mathrm{Q}$. Given this definition, two types of recommendations can be further subdivided, i.e. binding and non-binding recommendations. A binding recommendation is normative in that $\mathrm{P}$ assumes a position, in which $\mathrm{P}$ is to fault $\mathrm{Q}$ if $\mathrm{Q}$ fails to do what is being recommended to him, whereas a non-binding recommendation does not carry similar normative force. ${ }^{6}$ In other words, non-binding recommendations can be viewed as a form of suggestion, where a person's non-compliance shall not invite criticisms. It is the combination of

4 Here, the recommendation is formulated positively, i.e. it is to encourage a person to do something. It can also be formulated negatively to discourage a person from doing something. But, I shall only discuss positive recommendations in this chapter. Also see, Chapter 1, section 1.5.1.1.

5 For a discussion of good simpliciter and goodness-for, see Chapter 1, section 1.4.1.

6 It should be noted that there are, at least, two forms of binding recommendations, i.e. strong and weak binding recommendations. A strong binding recommendation involves legal and/or economic sanctions upon non-compliance, and a weak binding recommendation does not necessarily involve legal and economic sanctions. I assume the normativity of weak binding recommendation is primarily moral and prudential. In other words, a person's failure to comply with a weak binding recommendation should invite moral and/or prudential criticisms of the person, but not necessarily more than that.

Although the discussion of strong binding recommendations is an interesting topic for philosophical and policy debate and rightly under the scope of paternalism, this chapter mainly concerns with weak binding recommendations. Since offering weak binding recommendations is what is currently being done almost exclusively by technologists and gurus, and the aim of the present chapter is to urge philosophers to take up this task. Although weak binding recommendations may not be under the scope of paternalism in the strictest sense, the status of weak binding recommendations, i.e. whether it raises paternalistic worry or not, is directly related to the willingness of philosophers to offer recommendations to the public. 
binding recommendations and the placement of the person $\mathrm{Q}$ as $\mathrm{S}$ in (ii) that, $\mathrm{I}$ think, renders the practice of recommendation paternalistic.

Let's now turn to paternalism. There are various definitions of paternalism and/or criteria for a behaviour or practice to be counted as paternalistic (see, e.g. Garren 2006, 2007; Dworkin 2010; Grill 2012). So, whether offering recommendations is paternalistic or not effectively depends on one's definition and/or criteria. It is not my aim to provide an exact definition of paternalism. Rather, I want to explain why paternalistic behaviours and practices are deemed objectionable by looking at those definitions and criteria. Kalle Grill (2012) has helpfully suggested four parameters for defining paternalism, namely the interference condition, the consent condition, the benevolence condition and the superiority condition. ${ }^{7}$ One way or another, these conditions make paternalism problematic. In the following, I shall look at these conditions and explain why they create a sense of uneasiness. At the same time, I shall illustrate how the relevant type of recommendations, i.e. binding recommendation, fits those conditions, and so being paternalistic and prima facie undesirable.

The interference condition states that paternalism involves interfering with a person's course of action against the person's will in spheres of life that are under the person's legitimate control. In other words, paternalism involves interference of a person's "liberty and autonomy" (Dworkin 2010). Interference is typically interpreted as a closing of person's choices that are otherwise available to the person, or as a substitution of the person's judgement and agency by inducing certain decisions and/or actions. Note that, however, interference is not necessarily coercive, it can be achieved through deception, manipulation and incentivisation (Scoccia 2008; Thaler \& Sunstein 2008). The interference condition (and, by extension, paternalism) is seemingly objectionable because it clashes with our intuition that human beings are free and autonomous, at least for matters that lie squarely in spheres that are under a person's control. According to this intuition, people should be left alone for their rational decisions and actions. Otherwise, one is imposing a view of what is good and bad to that person, which the person does not necessarily agree. In this respect, a binding recommendation satisfies the interference condition because it does limit people's choices by imposing a (moral) cost to their non-compliance, or it replaces their judgement and agency by claiming

7 Cf. Dworkin (2010). 
that they should follow a specific decision and/or action regardless of their own will.

It is debatable whether a binding recommendation is genuinely paternalistic. For example, if a binding recommendation is accompanied by reasons to follow it, then it seems to become an attempt of rational persuasion, which few would label as paternalistic. It is so because in giving reasons to follow the (binding) recommendation, the person who gives the recommendation is facilitating better judgements of other people, and so helping them to truly realise their agency. It is, indeed, true that binding recommendations can be given in the form of rational persuasion. But, the question is whether or not rational persuasion is sufficient to be philosopher's duty. In other words, should philosophers stop at giving reasons for doing something? I think there is a significant difference between a binding recommendation and rational persuasion, and rational persuasion is not always the best way to convince the public, especially on issues about technology and the good life. Although I contend that rational persuasion may be a better way to convince the public to adopt technology in a specific way, rational persuasion appears to be insufficient to settle issues because of pluralism in values. Imagine, for example, a philosopher trying to convince the digital natives of the potential vices of Social Networking Sites, arguing that they will have adverse effects on their privacy, on the nature of friendship or on their good life in general, and the philosopher does so by giving prudential reasons to support the claims. Still, the digital natives can easily reject the philosopher's claim-not necessarily because the philosopher has made disputable factual claims - but because they hold a different view of the good life, which warrants their use of Social Networking Sites. In this scenario, holding on to rational persuasion requires the philosopher to leave the disagreement as it is, as long as the digital natives can give rational replies based on their view of the good life. In other words, philosophers appear to be inept to alter any behaviour and practice, which they see (un)desirable, if two or more fundamentally different views of the good life are in question. If philosophers want to be effective in encouraging and discouraging certain behaviours and practices, they have to be prepared for promoting or demoting a specific view of the good life, and even when rational persuasion fails, they should be prepared to praise (or condemn) what they see (un)desirable with respect to the view of the good life they hold.

In short, the weakness of rational persuasion with respect to technology and the good life, in comparison to binding recommendation, is that it requires an agreeable common ground if rational persuasion is to be successful at all. However, it is not entirely clear that such an agreeable common ground can be established for 
issues on the good life. It will be an ideal scenario if philosophers can convince the public simply through rational persuasion, but in reality there are different views of the good life that make it impossible. If philosophers hold on only to rational persuasion, they are bound to be silent when disagreements arise.

Of course, not all kinds of interference are objectionable. Particularly, it has been argued that if the person consents to the interference, then it is justifiable. The consent condition is about whether the person being intervened with consents to the interference or not. In a situation where the interference is not consented, it is known as a form of "hard" paternalism, whereas in a situation where the interference is consented, it is often labelled as "soft" paternalism. And, "soft" paternalism is typically accepted as unproblematic. ${ }^{8}$ Now, whether binding recommendations belong to "hard" or "soft" paternalism is to be determined by people's consent to philosophers who offer binding recommendations to them. Although it is theoretically possible to obtain consent from people receiving recommendations, it seems practically impossible to obtain consent prior to binding recommendations being offered to them. ${ }^{9}$

The benevolence condition states that paternalism is directed at a person's own good. In other words, it is the placement of the person as S in (ii) in my formulation of recommendation. The benevolence condition in itself is not objectionable, but it implicitly presupposes that a person $\mathrm{P}$, i.e. the person being paternalistic, is in a better position than another person $\mathrm{Q}$ to determine what is good and bad for $\mathrm{Q}$ thereby $\mathrm{P}$ is in a superior position to $\mathrm{Q}$ with respect to matters that belong to Q's legitimate control. Hence, paternalism exhibits a form of elitism-it satisfies the superiority condition. And, this is objectionable as it is considered to be an insult to an otherwise competent person, who is viewed as incapable of living his own life. Clearly, in offering a binding recommendation, a person $\mathrm{P}$ is committed to the superiority condition in that $\mathrm{P}$ is claiming that the person $\mathrm{Q}$ who has been offered the recommendation, ought to follow what P says, and Q's failure to do so will elicit criticisms from $P$.

\footnotetext{
8 See, Feinberg (1986). Feinberg's (1986) discussion of 'hard' and 'soft' paternalism, I think, is the most influential in the field.

9 Philosophers, of course, can offer binding recommendations on the assumption that there is hypothetical consent between them and the public, or to have subsequent consent from the public afterwards. As Van De Veer (1979) has pointed out, however, neither hypothetical consent nor subsequent consent is the same as actual consent.
} 
There is, however, one caveat to the superiority condition. Consider in what situation $\mathrm{P}$ is in a rightful position to instruct Q's decision and action. It seems that when $\mathrm{P}$ is an expert on the matters related to Q's decision and action, then $\mathrm{P}$ is in a rightful position to offer $\mathrm{Q}$ instruction. In other words, when $\mathrm{P}$ is an expert on the relevant issues, then $\mathrm{P}$ can rightly be in a superior position. Yet, whether there can be expertise on the issues pertaining to the good life is much disputed. It is often held that questions about the good life, i.e. how a person should live his life, are personal and subjective, and therefore no expertise-except for the person in question-is warranted (see, e.g. Crowley 2005; Steinkamp et al. 2008, pp. 175177). ${ }^{10}$ Hence, philosophers who offer (binding) recommendations to the public may risk being seen as groundless and idiosyncratic. It may be seen as groundless because there are no grounds for philosophers to recommend their own view(s) of the good life to the public in a binding manner, and it may be seen as idiosyncratic because it is only philosophers' own the view(s) of the good life but not necessarily applicable to the others. In short, the questionability of expertise on the good life has led to philosophers' hesitation to the practice of recommendation because of the superiority condition.

\subsection{Paternalism is Inevitable: An Inconvenient Truth}

If offering recommendations to the public can be viewed as paternalistic, and paternalism is objectionable, then philosophers are right to refrain themselves from doing so. However, if paternalism is inevitable, then perhaps philosophers should not preoccupy themselves with questions on why paternalism is objectionable or when it is justifiable, but to reconsider their role in light of its inevitability. In this section, I want to offer an argument against the possibility of non-paternalistic condition, thereby to urge philosophers to reconsider the prospects of the practice of recommendation. The argument, however, should not be misunderstood as an endorsement of paternalism. Nor should it be understood as an argument in favour of paternalism. This argument aims to show that paternalism is inevitable, and it is currently practised by designers and engineers (at the stage of design and production) and technologists and gurus (at the stage of application), and both

\footnotetext{
10 Note that the debate on moral expertise is typically centred on morality in the narrow sense, i.e. the right. I think the problem for expertise on the good life is even more acute than expertise on morality in the narrow sense, because it is more difficult to provide a (normative) standard to the expertise in question.
} 
groups of people are not the ideal candidates for the task. Philosophers' involvement, I think, will be a better alternative than them alone. My argument can be formulated as follows:

(P1) Non-paternalistic condition. People should be left alone in the spheres of life that are under their legitimate control (provided that no harm is inflicted on others). This means that a person should be able to live his life in accordance to his view of the good life, and no view of the good life should be imposed on the person.

(P2) Plurality of the good life. In the contemporary society, there are diverse, and possibly conflicting, views of the good life.

(P3) Technology is not neutral. There are values embedded in technology, and users of technology are necessarily influenced, constrained and/or shaped by it.

(P4) Technological design and production elitism. Technology is designed and produced by a small number of specialists, e.g. designers, engineers, producers, etc. Ordinary people, i.e. the public, cannot (always) be directly involved in technological design and production.

(P5) Mismatches of the good life. Given (P2), (P3) and (P4), there are bound to be mismatches between the views of the good life held by the users of technology —or, the public —and the values proffered by the technology, which are selected and implemented by elitists in technological design and production.

(PC) Inevitability of the paternalistic condition. Given (P5), (P1) is unattainable.

(P1) describes the condition in which people are free from interference on the matters that are under their legitimate control. And, this kind of interference is objectionable because it imposes a view of the good life-or, more accurately, a view on how people should live - on the people. By showing that such a condition is unattainable, I hope it can mitigate the uneasiness associated with paternalistic behaviours and practices. 
(P2) is generally accepted by liberal theorists. ${ }^{11}$ And, it is often cited in support of the importance of (P1), that is-if there are different views of the good life that are equally valid, then it appears that there is no good justification to impose a specific view of the good life on another person. Conversely, if there is only one, true view of the good life, then it seems that there is at least one ground to impose that view of the good life on the people, namely it is the true view of the good life.

(P3) has summarised one of the major the lessons we learnt from science and technology studies, i.e. technology is value-laden (e.g. Winner 1986; Brey 2010a). In a nutshell, this amounts to the claim that values are built into technology at the stage of its design and production by opening up certain options or making them more accessible to the users, and vice versa. And, users of technology are influenced, constrained, or shaped by the values proffered by the technology in question. In effect, I think (P3) in itself is sufficient to demonstrate the unattainability of (P1): if values are built into technology, and users of technology are influenced, constrained, and shaped by them, as long as the users are using the technology, they cannot escape from the values proffered by the technology being imposed on them. In other words, users will not be left alone by the technology in the spheres that are under their legitimate control. Still, I want to introduce an additional premise to illustrate the nature of the values being built into the technology in (P4).

I intend (P4), I hope uncontroversially, to be a statement of fact. In most technological design and production processes, users are conceived as passive receivers of technology, who are not and often cannot be involved in the processes. ${ }^{12}$ The passivity of users has an important implication to the present discussion, as stated in (P3), values are built into technology; and, the values are built into the technology by its designers and engineers, who claim special technical knowledge that are unavailable to the users. And, they are also a small group of people who oversee the design and production processes. In other words, users are 'instructed' how to live by the designers, engineers and others in the chain of production, who determine-either consciously or unconsciously-the values to be embedded in the technology and, relatedly, decide what is good and bad for the

\footnotetext{
11 In fact, ( $\left.P_{2}\right)$ is best viewed as a reformulation of Rawls's (1993) "the fact of reasonable pluralism".

12 There are other models of technological design and production in which users actively take part in the processes, most notably the (theory of) Participatory Design. See, e.g. Muller \& Kuhn (1993), Muller (2009) and Iversen et al. (2010). However, I believe ( $\left.\mathrm{P}_{4}\right)$ has captured the common model(s) of technological design and production processes.
} 
users. ${ }^{13}$ In this respect, designers and engineers cannot but impose a view of the good life to the users.

Finally, (P5) reiterates the fact that people have different views of the good life, which are equally valid, i.e. (P2). Given that technology is not neutral-values are built into technology either consciously or unconsciously, i.e. (P3), and that it is the designers, engineers and others in the chain of production, but not the users themselves, who determine the values to be embedded in the technology, i.e. (P4). There will be mismatches between the values upheld by the users and those proffered by the technology.

So long as people are users of technology, some values (or, a view of the good life) are imposed on them. So construed, (P1) is unattainable, i.e. (PC).

Two points of clarification should be added before proceeding. Firstly, to reiterate, this argument is not intended to be an argument in favour of paternalism. Or, it may not even be seen as an argument about paternalism in the strict sense. For instance, the values embedded (or omitted) in technology by the designers, engineers, and other related parties may not aim at the good of users, i.e. the public, but only at their own economic interest. That may well be so. But, it is irrelevant to the argument presented here. The argument here is to show that people cannot be free from values being imposed on them as long as they are users of technology, and thus to urge philosophers to reconsider their duty in light of its inevitability.

Secondly, it should be pointed out that my rejection of non-paternalistic condition resides on the assumptions that people do not opt out from being the users of technology, and that people cannot easily change from one technology to another in order to match it with their own values; it is so, as Brey (2000, 2010a) has argued that the values built into technology are often obscure to and hidden from ordinary users. Hence, it will be difficult for them to select and decide at will which technology to use to match their view of the good life. ${ }^{14}$ So construed,

\footnotetext{
13 The view of technology design and production being described here is admittedly oversimplified. For instance, other parties, e.g. managers and sales and marketing departments, have also played an important part in the design (and production) processes. I contend that the view being described is indeed over simplistic. However, the argument only requires the claim that ordinary people - the public- do not (and cannot) decide which values to be included (or omitted) in the technology.

${ }_{14}$ Indeed, I have formulated my argument in terms of technological artefacts, in which case people, i.e. the users of technological artefacts, can still practically decide whether
} 
philosophers should, minimally, try to disclose the values embedded in technology and inform the users and policy-makers, and enable them to adopt those technologies that suit their view of the good life best. For those who worry about paternalism, however, philosophers should only do so in a descriptive way without normatively affirming any values. But, why stop at descriptively informing users and policy-makers of the values embedded in technology, except because it raises a paternalistic worry? If paternalism engendered by technology is inevitable, then philosophers should not be afraid of taking up the challenges that emerge from the inevitability of paternalism.

\subsection{On Being a 'Paternalistic' Philosopher}

If the argument I have presented in the previous section is right, then paternalism engendered by technology is not something that philosophers can and should avoid. Instead of trying to convince themselves there are ways out of paternalism, philosophers should reconsider their role in light of its inevitability. One direction, as Jeroen van den Hoven (2008) has noted, is "the Design Turn in Applied Ethics", where philosophers start tinkering with the values embedded in socio-technical systems and technological artefacts through their design and production processes. I think the Design Turn is a fruitful pursuit, but I also think that the turn alone is insufficient to meet the objective it sets for itself. I shall argue that design ethics has to be supplemented by the practice of recommendation if it is to attain its own objective.

\subsubsection{Paternalism by Design}

In line with the Design Turn, Verbeek (2006, 2008, 2010) has developed and elaborated Hans Achterhuis's notion of "moralisation of technology", which urges ethics of technology to "engage in the development of [technology] that helps to form moral action and decision-making" (Verbeek 2008, p. 101). Similarly, the theory of Value Sensitive Design (VSD) is intended to be "a theoretically grounded approach to the design of technology that accounts for human values in a principled and comprehensive manner throughout the design process" (Friedman et al. 2008,

to have (and use) them or not. However, a stronger version of the argument against nonpaternalistic condition can be formulated in terms of technological systems (or sociotechnical systems), in which case people have few-or, even no-authority over them. 
p. 69; see also, Friedman \& Kahn 2007). Either way, in accordance to the Design Turn, philosophers should be actively involved in the design and production processes by helping designers and engineers to select and decide the values to be embedded (and omitted) in technology. In this sense, the Design Turn is essentially paternalistic too. ${ }^{15}$

All is good to philosophers who accept the challenge of the Design Turn. As I have already shown that paternalism engendered by technology is unavoidable, it should not deter philosophers from participating and intervening in the design and production of technology. In short, one of the major objectives of the Design Turn is to embed desirable values (or to omit undesirable values) in technology through its design (and during its production). To meet this objective, however, two important questions have to be answered by philosophers of the Design Turn:

1. What values should be embedded (and omitted) in the technology? This question is both methodological and normative. Methodologically, philosophers, together with designers and engineers, need to formulate a method to search for the values to be included in (and excluded from) technology. Normatively, they have to determine what values are right, just and/or good to be embedded in the technology.

2. How to bridge the gap between the designer's intention and actual use? Anders Albrechtslund (2007) has argued that VSD and other similar design ethics face a problem he called "the positivist problem". Briefly, the problem arises from the possibility of users departing from designer's intention in actual contexts of use. Hence, the designer's intention and actual contexts of use must be somehow be (re)integrated if positive contributions from the Design Turn are to be ensured.

To the first part of (1), researchers of VSD have formulated a "tripartite methodology" that incorporates conceptual, empirical and technical investigations into their analyses (Friedman et al. 2008, pp. 71-73). Explicit attempts have also been made to apply VSD to ethical issues (e.g. Cummings 2006). However, it has been argued that the current works in VSD are inadequate for the second part of (1). Particularly, it has been noted that VSD in itself lacks the resources to make normative judgements for designs because it is not explicitly aligned with any

15 Thaler and Sunstein's (2008) Nudge is another example of 'paternalism by design'. But, unlike Verbeek's view and the theory of Value Sensitive Design, their account is motivated by what they believe to be an inadequacy of human rationality. 
normative theories (Albrechtslund 2007, Manders-Huits 2011). ${ }^{16}$ And, to (2), Albrechtslund's own proposal is to accept the distinction between the context of design and the actual contexts of use, and to acknowledge the limited role designers can have on promoting (and demoting) the values to the public. However, the objective of the Design Turn becomes defeasible if users can easily deviate from the intended use. Yet, the distance between a designer's intention and the actual contexts of use is inherent to the use of technology. As such, philosophers of the Design Turn cannot limit themselves with design alone if their aim is to promote the good life through the design of technology. They must also be prepared to argue for the proper usage in terms of the good life the technology aims to promote. However, these kinds of arguments will be based on a specific view of the good life, and the users of technology can rationally disagree with philosophers on the claims about the good life. In keeping with the objective of the Design Turn, therefore, philosophers need to find a way to defend the design and the intended use. Since the gap between the intended use and the actual contexts of use cannot be bridged at the stage of design and production, nor can it always be achieved through rational persuasion, binding recommendations offer a valuable option for philosophers to defend the design and close the gap between the intended and actual use of technology.

In short, philosophers of the Design Turn need to (re)connect design ethics with normative ethical theories - and, for the present purpose, views of the good life - and to defend the design (and, in a similar vein, the technology) through their favourite normative theories.

\subsubsection{Design, Recommendation and the Walzerian Approach}

I have identified two questions for philosophers of the Design Turn, and I have also tried to show that the practice of (binding) recommendation should be an integral part of the Design Turn. There are, of course, different ways to conceive binding recommendations, and, relatedly, there are different ways to offer them to the public. Building on Michael Walzer's idea of social criticism and his notion of thick morality, I have proposed a Walzerian approach to digital media and the good life. I

\footnotetext{
16 In her paper, Manders-Huits (2011) has also identified problems in VSD's methodology, particularly VSD's notion of stakeholders and the relations between the conceptual, empirical and normative investigations within VSD. I will not answer them here, but will return to them below.
} 
think the Walzerian approach can too provide a satisfactory answer to the aforementioned questions, and mitigate the paternalistic worry from the practice of (binding) recommendation. ${ }^{17}$

First, to the question about the connection between design ethics and normative ethical theories, the Walzerian approach takes the users' values as a starting point and, it scrutinises and (re)interprets them using the resources within the user's culture. Accordingly, the resources to make normative judgements are already there in the culture, and they are being expressed in public discourse. This is exactly why it is important that philosophers ought to participate in the public discourse and engage with the public, who are themselves interpreters of values. It is by virtue of their participation and engagement that philosophers derive those values to be embedded (or omitted) in technology that are truly at the heart of users. In short, the Walzerian approach searches for values in the public discourse, and it scrutinises and (re)interprets them together with the users, i.e. the public. In this way, philosophers can become a proxy of the reflected public and supply valuable inputs to designers and engineers. ${ }^{18}$

More importantly, perhaps, is its answer to the second question. I have already argued that if the objective of the Design Turn is to promote (or demote) certain values that will enhance people's lives, then the gap between the designer's intention and the actual contexts of use must be bridged. To do this, philosophers must be prepared to stand up for the values embedded (or omitted) in technology. ${ }^{19}$ And, offering binding recommendations is one effective way to do so. I have also tried to show that philosophers should not be afraid of offering binding recommendations because of the paternalistic worry it raises. Still, the related

17 See, Chapter 1, section 1.1.2.

18 It is, in fact, very similar to "empirical investigations" in VSD's tripartite methodology. But, the current approach differs from it in that (i) it insists that "empirical investigation" should be the starting point of normative analysis and (ii) that researchers are not observers, who take a distance from the subjects, but are participants, who are deeply involved and engaged in the discussion of values.

19 Of course, not all deviations from the intended use is bad by definition. Sometimes, a deviation from an intended use can realise important values for the users. The Walzerian approach proposed here, in virtue of its participatory nature, can also take that into account. In this scenario, philosophers should scrutinise and (re)interpret the values realised by the deviation, and to help designers and engineers to incorporate them into the future design. 
worry about elitism appears to persist, namely on what (superior) grounds are philosophers warranted to tell people how they ought to live their own lives?

On this worry, there is an immediate response available to the Walzerian approach. In this approach, philosophers, working with designers and engineers, should only embed those values which the users themselves endear. Since the selected values are derived from philosophers' reflection and interpretation, and revised and buttressed through their participation in and engagement with the public, the values-being values of the users-provide a legitimate ground for instructions and criticisms. Also, recall the worry about elitism arises from the superiority condition. Elitism from the practice of (binding) recommendation is deemed problematic because it is argued that there is no expert of the good life, and thus telling people how they should live their own life with binding recommendations will be an insult to them. But, the Walzerian approach does in fact allow philosophers to acquire a form of expertise on the good life. Since philosophers reflect on and (re)interpret existing views of the good life with the public through participation and engagement, it should be relatively uncontroversial to claim that they are-or, can be-experts of the existing views of the good life. In this respect, the Walzerian approach provides a more solid ground for philosophers to defend the values they seek to embed through design. After all, those values are users' own values. At the same time, through the Walzerian approach's connectedness, philosophers can understand better and more immediately the user's deviations from the designer's intention. After all, those values are users' own values. At the same time, through the Walzerian approach's connectedness, philosophers can understand better and more immediately the user's deviations from the designer's intention and, in return, allow philosophers to respond to them-especially to the positive deviations-more timely and appropriately.

\subsection{Conclusion}

I begin this chapter by identifying two types of recommendations, i.e. binding and non-binding recommendations. And, I suggest that the practice of (binding) recommendation is susceptible to the charge of paternalism, which is objectionable to many. I then present an argument showing that paternalism engendered by technology is inevitable, and is currently practised by designers, engineers and others in the chain of production. In light of this, I attempt to urge philosophers and ethicists to reconsider their role in light of its inevitability. In this respect, philosophers of the Design Turn are the group of researchers who accept the 
inevitability of paternalism engendered by technology and take up the opportunities and challenges it creates by being more proactive in the moral and prudential dimensions of (the design of) technology.

Still, there are two questions for philosophers of the Design Turn, namely (i) the methodological and normative question on the relationship between values and the design of technology and (ii) the gap between the designer's intention and the actual contexts of use. Particularly, I have pointed out that if the objective of the Design Turn is to be realised, philosophers must bridge the gap between the designer's intention and actual contexts of use. I suggest that the Walzerian approach developed and elaborated in the present study provides an answer to (i) and (ii). I point out that existing morality and views of the good life offer abundant resources for philosophers to justify the selected values to designers and engineers, as well as the public. This approach also allows philosophers to be in a right position to instruct the users and to criticise negative deviations from the designer's intention. At the same time, it should allow them to help designers and engineers to adopt positive deviations in the future design.

I want to conclude this chapter by reminding what it is not about. First, it is not about paternalism in the strictest sense. Here, my aim is only to argue that if philosophers think that offering binding recommendations to the public is paternalistic and thereby objectionable, they should not do so. Since philosophers are in a better position - at least, according to the Walzerian approach - to examine the good life, they should fare better than designers and engineers, who do not necessarily pay enough attention to moral and prudential dimensions of (the design of) technology, in response to the inevitability of paternalism. Second, it is also not a paper on design ethics. While I have discussed design ethics here, I only want to point out that design ethics, which is one of the best responses to the inevitability of paternalism, is insufficient without the practice of (binding) recommendation. In short, it is an attempt to reassert the importance of (binding) recommendation, which has largely disappeared from recent works in philosophy and ethics of technology.

However, even if the view present here is theoretically justified, its practical feasibility remains to be proven. Particularly, whether and how philosophers will be heard by designers and engineers and the public has to be further investigated. I have not provided any solutions to practical problems associated with the practice of recommendation. However, it is clear that philosophers, in order to collaborate with designers and engineers and to communicate with the public, need to develop 
different skill sets. Yet, if the view presented here is correct, i.e. philosophers are needed for the good of the public, then it is our duty to reinvent ourselves to take up the opportunities and challenges. 


\section{EPILOGUE}

The project of Net recommendation has been to (re)assert the importance of actual discourses in normative analysis of digital media and the good life, as they provide valuable insight into the normative and axiological foundation of our normative judgements on digital media and digitally-mediated practices and allow us to better offer practical recommendations to the public. In order to incorporate prudential appraisals in normative analysis of digital media and the good life, I have introduced a Walzerian approach to digital media and the good life, which is based on Walzer's idea of social criticism (i.e. in Chapter One). The Walzerian approach is (i) hermeneutical, (ii) immanent, (iii) participatory, (iv) empirical and (v) pluralistic. And, it calls for taking seriously the existing views of the good life expressed in actual discourses, and to respond to (and, ideally, interact with) them.

In the subsequent chapters, I have applied the Walzerian approach, together with different notions of the self I have identified (i.e. in Chapter Two and Chapter Four), to (re)interpret a number of representative examples of prudential appraisals in an intra-cultural context (i.e. in Chapter Three), as well as China's Internet policy and a set of opinion pieces in a major newspaper in China in an intercultural context (i.e. in Chapter Five). I hope these analyses have demonstrated the importance of actual discourses (i.e. prudential appraisals) in normative analysis of the relations between digital media and the good life at both the local level and the global level, as well as the ways in which they can shed light on our normative judgements on digital media and digitally-mediated practices. 
Finally, in Chapter Six, I have dispelled one serious worry over the project of Net recommendation and the Walzerian approach, i.e. the practice of recommendation being paternalistic. I have shown that paternalism is inevitable in our technologically-mediated lives, and thus we should not worry so much about paternalism per se, but instead we should take up the opportunities and challenges result from its inevitability. At the same time, I have also shown that Walzerian approach in effect provides researchers a better option to minimise the worry of paternalism in a technologically-mediated world.

Together, I hope, I have provided an adequate discussion and defence-in the guise of the Walzerian approach-of the project of Net recommendation, i.e. a balanced and constructive way to examine the relations between digital media and people, particularly the relations between digital media and the good life, that does not start with the assumption of digital media being a source of moral problem and strives to recommending specific ways to reform and/or transform digital media and digitally-mediated practices that allow us to have better relations with digital media, and thus help us to live better lives with it.

The project of Net recommendation, however, is far from complete. Particularly, the focus of the present study is exclusively on the relations between digital media and the good life. In other words, I have only analysed the impacts of digital media on individuals. However, the impacts of digital media go beyond the level of individuals, as my analysis of prudential appraisals in Chapter Three has already hinted, digital media has transformative impacts on our society, economy, education, etc. as well. As such, the project of Net recommendation has to include the topics of the good society, the good economy, the good education, etc. too. After all, the aim of the project is to reform and transform digital media and digitallymediated practices for a better world.

Fortunately, I think the Walzerian approach has already provided the basic building blocks for this task. In my analysis, I have shown that the self is the normative and axiological foundation of prudential appraisal of digital media. I believe the Walzerian approach can be extended to normative analysis of digital media and the good society, the good economy, the good education, etc. It is true that whether the notion(s) of the self by itself suffices for analysing actual discourses of other subjects require further scrutiny, but the Walzerian approach should provide a general framework to analyse them.

At the same time, I have implicitly assumed that it is the responsibility of philosophers and researchers to advance the project of Net recommendation 
throughout this study. I have held this assumption because I conceive the project to be a bottom-up effort, i.e. the key is to reforming and transforming digital media and digitally-mediated practices with the people, by the people. The role of philosophers and researchers, therefore, is to facilitate public reflection through their participation in public discussion and their engagement with the people, and to help realise their (or our) visions of the good life. In short, the project of Net recommendation also calls for a revival of public intellectualism. In sociology, this point has been made by Michael Burawoy (2005), who has proposed a branch of sociology he calls 'public sociology' to promote sociologists' public engagement and argue for its importance. Similarly, Wiebe E. Bijker (2003) has urged STS scholars to step up as public intellectuals. I believe the same is needed from researchers in the field of ICE and critical studies of digital/new media.

However, it is important to note that the reflection on the role of researchers of digital media in public cannot merely be a form of self-reflection. Insofar as we are members of academia, which is located in the institutional structure of society, the plausibility and success of public intellectualism is also rested on an institutional structure which enables and favours our participation and engagement. This, in turn, also calls for a critical reflection on the (existing) institutional structure of academia. For example, education and training, as well as motivation and incentive (e.g. in the forms of academic/tenure review, research funding, etc.), should be provided to participation and public engagement (see, e.g. Stevens 2008).

Despite my conviction that the project of Net recommendation should be a bottom-up effort, it is impossible to deny the role of the government and policymakers in shaping our digitally-mediated world, and thus shaping our digitallymediated lives. Hence, before closing this study, it is appropriate to discuss some (more) practical implications of the present study at the policy level. I should point out that, however, the following discussion can only be brief and speculative, because further works must be done to fully explicate the practical import this study.

I have already argued that in our digitally-mediated world (or technologicallymediated world), there is no escape from paternalism engendered by technology, i.e. having some views of the good life being imposed on us. ${ }^{1}$ Insofar as the government and policy-makers are responsible for setting agendas and policies on technology, it is impossible to demand them to entirely refrain from interfering with our lives (at

1 See, Chapter 6, section 6.2. 
least, through the development and diffusion of technology), as agendas and policies on technology are not value-neutral. This result is related to thorny issues of (i) whether the government and policy-makers should (or, at least, are justified to) promote certain views of the good life through agenda-setting and policy-making; and, (ii) if the government and policy-makers should (and, are justified to) promote certain views of the good life through their agendas and policies, how should they do it.

For those who are convinced by the present study, the answer to (i) should be obvious, namely the government and policy-makers should (or, at least, are justified to) promote certain views of the good life through agenda-setting and policymaking. Indeed, the government and policy-makers cannot but to promote some ways of the good life and demote others in their agenda-setting and policy-making. However, it does not mean that the government and policy-makers can (or should) only promote one view of the good life, but it does mean that they can (or, indeed, should) promote the views of the good life to people who share them. In order to do so, the government and policy-makers must first understand people's views of the good life. In this respect, this study has attempted to show that our understanding of the views of the good life held by the people should be understood via different notions of the self and the ideals they embody. Although the notions of the self (and the ideals embodied in them) may appear to be unrelated to agenda-setting and policy-making at first glance, they are nevertheless central to our understanding of different views of the good life, and thus they are essential for issues concerning people's well-being. In light of this, it is important for the government and policymakers to (re)turn to the question of 'who we, i.e. the people, want to be' (and, 'who we, i.e. the people, should be') in their agenda-setting and policy-making.

The foregoing discussion has shown how (ii) can be answered as well, namely in promoting certain views of the good life, the government and policy-makers should base their agendas and policies on the views of the good life held by the people, and target them at those who share the views of the good life in question. This position is recently expressed by Dan Haybron and Valerie Tiberius (2012) using the label 'pragmatic subjectivism of well-being-based policy', i.e. "policies aimed at bettering people's lives must do so according to the beneficiaries' own standards; they must not impose some external standard of well-being on people" 
(Haybron \& Tiberius 2012, p. 6). ${ }^{2}$ Pragmatic subjectivism, as they argue, provides a tenable approach to well-being policy which preserves the importance of wellbeing in agenda-setting and policy-making and, at the same time, allows the government and policy-makers to respect people (and their sovereignty) in their agendas and policies. It is clear that Haybron and Tiberius's pragmatic subjectivism is in line with the Walzerian approach proposed in this study. Particularly, both of them emphasise the importance of the views of the good life held by the people. More importantly, however, is that Haybron and Tiberius's emphasis on "the beneficiaries' own standards", I think, has highlighted the insufficiency of any subject-independent (and/or context-independent) indices for well-being policy. If the government and policy-makers are genuinely interested in promoting wellbeing in terms of the views of the good life actually held by the people, well-being policy must be based on (or supported by) experientially rich information. This, I think, serves as a timely reminder for the information gathered from participation and engagement. ${ }^{3}$ In short, agendas and policies on technology that aim to promoting well-being should be based on and supported by people's experience. Accordingly, the government and policy-makers are required to (re)introduce our real experience in their agenda-setting and policy-making.

I want to end by quoting from Neil Postman (1992) whose remarks have inspired this study:

"Anyone who practices the art of cultural criticism must endure being asked, What is the solution to the problems you describe? Critics

2 Haybron and Tiberius (2012) distinguish carefully their pragmatic subjectivism from substantive subjectivism, where the former is not committed to a specific view of the nature of well-being, and the latter is committed to the view that the nature of well-being is subjective. Their pragmatic subjectivism is subjective in the sense that it concerns with the views of well-being held by the subjects, which, in turn, can be either subjective or objective in nature.

3 Haybron and Tiberius's (2012) solution is to consider multiple indices for well-being policy. Although I am sympathetic to their solution, I am generally sceptical to the use of indices of well-being as the only guide for agenda-setting and policy-making. Here, I cannot offer a full argument for the insufficiency of common indices of well-being as the guide for well-being policy, but it is useful to point out that those indices are often thin, i.e. they are abstract and generalised. Accordingly, the use of common indices of wellbeing suffers from the same problem as theories of well-being I have described in Chapter 1, section 1.6. Yet, it does not mean that pragmatic subjectivism is incompatible with the present study, especially the Walzerian approach. 
almost never appreciate this question, since in most cases, they are entirely satisfied with themselves for having posed the problems and, in any event, are rarely skilled in formulating practical suggestions about anything. This is why they became cultural critics.

The question comes forth nonetheless, and in three different voices. One is gentle and eager, as if to suggest that the critic knows the solutions but has merely forgotten to include them in the work itself. A second is threatening and judgemental, as if to suggest that the critic had no business bothering people in the first place unless there were some pretty good solutions at hand. And a third is wishful and encouraging, as if to suggest that it is well known that there are not always solutions to serious problem but if the critic will give a little thought perhaps something constructive might come from the effort."

Admittedly, I have not provided any 'fool proof' answers to the questions concerning the relations between digital media and the good life, but I hope I have provided something constructive to our answering of them. 


\section{SUMMARY}

Digital media has become an integral part of people's lives (at least, for those who live in the developed world), and its ubiquity and pervasiveness in our everyday lives raise new ethical, social, cultural, political, economic and legal issues. Although many of these issues have already been taken up by researchers, they are primarily being dealt with in terms of what is 'right' or 'just' with digital media and digitally-mediated practices. And, questions about the relations between digital media and the good life are often left in the background. In other words, what is often missing is an explicit discussion of the relations between digital media and the good life, especially in a more balanced and constructive manner.

Under the label of 'Net recommendation', the present study aims to offer a more balanced and constructive normative analysis of digital media, focusing on the relations between digital media and the good life. The project of Net recommendation aims to (re)assert the importance of actual discourses in our normative analysis of the relations between digital media and the good life. In the present study, I pursue the project of Net recommendation with a Walzerian approach to digital media and the good life that takes seriously (and, ideally, also interacts with) actual discourses. This approach, as I shall argue, allows us to have a better understanding of our normative judgements on the impacts of digital media has (or will have) on the good life and, at the same time, allows us to answer the question of 'how should we live with digital media?' more adequately. At the most general level, therefore, I hope the present study will contribute to the field of Information and Computer Ethics and critical studies of digital/new media by (re)turning to the good life through an analysis of the relations between digital media and the good life.

Yet, the Walzerian approach is not only useful to normative analysis of digital media and the good life in an intra-cultural context, i.e. the critical study of the impacts of digital media on the good life within a culture, it is also useful in an intercultural context. Using China's Internet as a case study, I show that the relations between digital media and the good life in each culture have to be 
examined in its own right. In short, the Walzerian approach is useful not only at a local level, but also at a global level. In this way, I hope the present study will add to the growing body of research in intercultural (and cross-cultural) studies of digital media.

In Chapter One, I introduce my approach to normative analysis of digital media and the good life, which is based on Michael Walzer's idea of social criticism. This approach is characterised by five features, i.e. (i) hermeneutical, (ii) immanent, (iii) participatory, (iv) empirical and (v) pluralistic. It takes seriously the actual discourses (e.g. popular discourse) on digital media and urges researchers to integrate them into their normative analysis. Since the focus of my study is on the relations between digital media and the good life, I identify a specific type of actual discourses on digital media that is relevant to the present study, i.e. prudential appraisals of digital media. Here, I argue that prudential appraisals of digital media are normative, and that their normativity is grounded in our practical identity (or our self-interpretation and self-understanding). I discuss how, and in what sense, practical identity is the source of normativity, and point out what this means to normative analysis of digital media and the good life. Finally, I end the chapter by offering an additional argument for the indispensability of prudential appraisals of digital media in normative analysis of digital media and the good life.

As I argue in Chapter One, prudential appraisals of digital media are normative and their normativity comes from people's self-interpretation and selfunderstanding. In order to properly understand our normative judgements on the impacts of digital media on the good life, it is necessary first to explicate the normative ground(s) behind the judgements, i.e. our mode(s) of self-interpretation and self-understanding. In Chapter Two, through a discussion of the works of Charles Taylor, Anthony Giddens and Ulrich Beck, I identify three different notions of the self in modern and late modern societies that serve this purpose, i.e. the disengaged self, the expressive self and the reflexive self; and, at the same time, I also identify the ideals (or the views of the good life) these notions of the self embody. Finally, I support my characterisation of the views of the good life in modern and late modern societies by looking at the empirical research conducted by Ronald Inglehart and his colleagues. The main objective of this chapter, in short, is to lay the ground for my analysis of prudential appraisals of digital media in the next chapter by recollecting the normative and evaluative resources for (re)interpreting and understanding them.

In Chapter Three, I apply the Walzerian approach with the notions of the self I discuss in Chapter Two to prudential appraisals of digital media. I examine two 
sets of prudential appraisals of digital media: the first set focuses on the impacts of digital media on culture and society, which influence people's lives by transforming the exteriors of their lives; and, the second set focuses on the impacts of digital media on our brain, mind and/or the self, and how it influences people's lives by transforming their interior lives. More specifically, I attempt to show that prudential appraisals of digital media-and, for that matter, any normative judgements on the impacts of digital media on the good life-are best understood with the notion(s) of the self. This, I also attempt to show, has an important implication to normative analysis of digital media and the good life, namely we should (re)direct our attention to the question of "who we should be in a digitallymediated world?'

Chapter Four is devoted to explore a different notion of the self, i.e. the Confucian self in (contemporary) China. I argue that the modern and late modern self I discuss in Chapter Two are inadequate for a comprehensive normative analysis of digital media and the good life, because they are not readily applicable to societies that have a different cultural root, and thus a different trajectory of modernisation. I propose that we should move beyond the idea of singular modernity and replace it with the idea of plural modernities, which allows us to properly acknowledge the importance of various cultural roots. With the idea of plural modernities in place, I explore the Confucian self and the view of the good life it embodies. Similar to what I have done in Chapter Two, the aim of this chapter is to lay the ground for my analysis of prudential appraisals of digital media in (contemporary) China by exploring the normative and evaluative resources available in the Confucian tradition.

I have applied the Walzerian approach in an intra-cultural context in Chapter Three, but the approach is also useful in an intercultural context. In Chapter Five, I illustrate how the Walzerian approach can be applied at a global level. I analyse the Chinese Communist Party's position on the Internet with the Confucian self, and illustrate the fundamental role of Confucian values (and the Confucian view of the good life) in grounding China's Internet policy and normative judgements in the opinion pieces. I argue that if it is indeed true that China's Internet is informed by a different normative and axiological foundation, i.e. the Confucian self, then the question of 'whether the Internet is good or bad?', or the question of 'how we should live with digital media?', should also be answered differently-in a socially and culturally sensitive manner. To illustrate this, I offer a discussion of social media from the Confucian perspective. I show that there is a prima facie incompatibility between the Confucian way of life and social media, but I also point 
out that the incompatibility between them may be resolved. In short, the lesson of this chapter is that different societies require their own normative analysis of digital media with their own evaluative and normative resources.

Implicit in the project of Net recommendation and the Walzerian approach to digital media and the good life is philosophers' responsibility to proactively offer practical recommendations to the public, i.e. users of digital media. However, the practice of recommendation is often faulted as paternalistic, and thus is considered to be morally undesirable. This criticism must be answered if the project of Net recommendation or the Walzerian approach is to be considered as a (morally) feasible option. In Chapter Six, I look at this criticism more closely. I argue that offering recommendations is indeed paternalistic, but we should not see it as morally problematic, because paternalism is inevitable in our technologicallymediated lives. Hence, philosophers should not be shied away from the practice of recommendation-especially only if it is for the worry over paternalism. Although there are philosophers who already take seriously the inevitability of paternalism in our technologically-mediated lives via the idea of design ethics, I point out that it must too be supplemented by the practice of recommendation. And, I illustrate how the Walzerian approach may supplement design ethics, as well as how it can actually minimise the worry over paternalism from the practice of recommendation.

Finally, in the Epilogue, I offer a brief summary of the present study and briefly discuss several practical implications of the project of Net recommendation and the Walzerian approach.

Together, I hope, this study have provided an adequate illustration and defence-in the guise of the Walzerian approach-of the project of Net recommendation, i.e. a balanced and constructive way to examine the relations between digital media and people, particularly the relations between digital media and the good life, that does not start with the assumption of digital media being a source of moral problem and strives to recommending specific ways to reform and/or transform digital media and digitally-mediated practices that allow us to have better relations with digital media and enable us to live better lives with them. 


\section{SAMENVATTING}

Digitale media zijn een integraal onderdeel geworden van het leven van mensen (in ieder geval voor diegenen wonend in de ontwikkelde wereld), en de vergaande alomtegenwoordigheid hiervan in ons dagelijks leven roept nieuwe ethische, sociale, culturele, politieke, economische en juridische kwesties op. Hoewel veel van deze problemen al onderwerp van studie zijn voor onderzoekers, worden ze in hoofdzaak bestudeerd in termen van wat 'goed' of 'rechtvaardig' is aan digitale media en digitaal gemedieerd gebruik. Daarnaast blijven vragen over de relatie tussen digitale media en het goede leven vaak op de achtergrond. Met andere woorden, wat vaak ontbreekt is een expliciete bespreking van de relaties tussen digitale media en het goede leven, vooral in een meer evenwichtige en constructieve manier.

Onder het label van 'Net recommendation' (Netwerk aanbeveling) heeft deze studie tot doel een meer evenwichtige en constructieve normatieve analyse van digitale media te bieden, geconcentreerd op de relatie tussen digitale media en het goede leven. Het project 'Net recommendation' heeft ten doel het belang van de actuele discours te (her)bevestigen in onze normatieve analyse van de relaties tussen digitale media en het goede leven. Het onderzoek in 'Net reccomendation' streeft ernaar een Walzeriaanse benadering toe te passen op digitale media en het goede leven, waarin actuele discours serieus genomen wordt (en waar idealiter ook een wisselwerking ontstaat met de discours hieromtrent). Deze aanpak, zoals ik zal betogen, stelt ons in staat om een beter begrip te hebben van de normatieve oordelen over de impact die digitale media heeft (of zal hebben) op het goede leven, en maakt het ons tevens mogelijk een adequater antwoord te formuleren op de vraag ' hoe moeten we leven met digitale media?'. Op algemeen niveau hoop ik daarom dat dit onderzoek een bijdrage zal leveren op het gebied van 'Information and Communication Ethics' en kritisch onderzoek naar digitale/nieuwe media door (terug) te keren naar de vraag van het goede leven, door middel van een analyse van de relaties tussen digitale media en het goede leven . 
Toch is de Walzeriaanse aanpak niet alleen bruikbaar voor de normatieve analyse van digitale media en het goede leven in een intra-culturele context, d.w.z. de kritische studie van de effecten van digitale media op het goede leven binnen een cultuur; ze is ook bruikbaar in een inter-culturele context. Met China's internet als case study zal ik laten zien dat de relaties tussen digitale media en het goede leven binnen elke cultuur op zichzelf moeten worden onderzocht. Kortom, de Walzeriaanse aanpak is niet alleen bruikbaar op lokaal niveau, maar ook op mondiaal niveau. Op deze manier hoop ik dat dit onderzoek zal bijdragen aan de groeiende hoeveelheid onderzoek in de interculturele (en cross-culturele) onderzoeken gericht op digitale media.

In hoofdstuk een introduceer ik mijn benadering voor normatieve analyse van digitale media en het goede leven, gebaseerd op het idee van Michael Walzers' sociale kritiek. Deze benadering wordt gekenmerkt door vijf eigenschappen, namelijk (i) hermeneutisch, (ii) immanent, (iii) participatief, (iv) empirisch en (v) pluralistisch. Deze benadering geeft actuele discussies (bijv. populaire discussie) over digitale media een serieuze rol en dringt er bij onderzoekers op aan om deze te integreren in hun normatieve analyse. Omdat de focus van mijn onderzoek ligt op de relaties tussen digitale media en het goede leven identificeer ik een specifiek soort van de actuele discussie over digitale media die relevant is voor het huidige onderzoek, dat wil zeggen prudentiële beschouwingen van digitale media. Dit hoofdstuk argumenteert dat prudentiële beschouwingen van digitale media normatief zijn, en dat hun normativiteit is gebaseerd op onze praktische identiteit (of onze zelf-interpretatie en zelf-inzicht). Ik bespreek hoe, en in welke zin, praktische identiteit de bron is van normativiteit, en maak duidelijk wat dit betekent voor normatieve analyse van digitale media en het goede leven. Tot slot wil ik het hoofdstuk afsluiten met een aanvullend argument voor de onmisbaarheid van prudentiële beschouwingen van digitale media in normatieve analyse van digitale media en het goede leven.

Zoals ik in hoofdstuk een beargumenteerde, behoudende beschouwingen van digitale media zijn normatief en hun normativiteit komt voort uit menselijke zelfinterpretatie en zelf-inzicht. Om onze normatieve oordelen over de impact van digitale media op het goede leven goed te kunnen begrijpen is het noodzakelijk om eerst de normatieve basis hiervan expliciet te maken, d.w.z. onze wijze(n) van zelfinterpretatie en zelfinzicht. In hoofdstuk twee identificeer ik drie verschillende opvattingen van 'zelf' in de moderne en laatmoderne samenlevingen die dit doel dienen, dat wil zeggen de ontkoppelde zelf, de expressieve zelf en de reflexieve zelf, met behulp van een bespreking van de werken van Charles Taylor, Anthony 
Giddens en Ulrich Beck. Tegelijkertijd identificeer ik ook de idealen (of de opvattingen van het goede leven) die deze begrippen van het zelf belichamen. Tot slot ondersteun ik mijn karakterisering van de opvattingen van het goede leven in de moderne en laatmoderne samenlevingen door te kijken naar het empirisch onderzoek van Ronald Inglehart en zijn collega's. Het belangrijkste doel van dit hoofdstuk, kortom, is de grond te leggen voor mijn analyse van de prudentiële bschouwingen van digitale media in het volgende hoofdstuk door de in hoofdstuk twee verzamelde normatieve en evaluatieve middelen samen te brengen voor (her)interpretatie en begripsvorming.

In hoofdstuk drie pas ik de Walzeriaanse benadering toe, met gebruik van de noties van 'zelf' die ik heb besproken in hoofdstuk twee, op prudentiële beschouwingen van digitale media. Ik onderzoek twee combinaties van de prudentiële beoordeling van digitale media: de eerste combinatie richt zich op de impact van digitale media op cultuur en samenleving, die het leven van mensen beïnvloeden door het transformeren van de externe aspecten van hun leven; de tweede combinatie richt zich op de impact van digitale media op onze hersenen, geest en/of het concept 'zelf', en hoe dat het het leven van mensen beïnvloedt door verandering van hun interne leven. Meer specifiek probeer ik aan te tonen dat prudentiële beschouwingen van digitale media-en overigens elke normatieve uitspraak over de effecten van digitale media op het goede leven-het best begrepen kunnen worden vanuit de ideeën van het concept 'zelf'. Ik heb ook proberen aan te tonen dat dit een belangrijke implicatie heeft voor normatieve analyse van digitale media en het goede leven, namelijk dat we (hernieuwd) onze aandacht moeten richten op de vraag 'wie zouden wij moeten zijn in een digitaalgemedieerde wereld?'

Hoofdstuk vier is gewijd aan het onderzoeken van een andere opvatting van het concept 'zelf', namelijk de Confucianistische zelf in (hedendaags) China. Ik betoog dat de moderne, zelfstandige en de laat-moderne concepten van 'zelf' zoals besproken in hoofdstuk twee ontoereikend zijn voor een uitgebreide normatieve analyse van digitale media en het goede leven, omdat ze niet eenvoudig kunnen worden toegepast op samenlevingen die andere culturele wortels hebben, en dus een ander traject van modernisering hebben gevolgd. Ik stel voor dat we het idee van singulaire moderniteit moeten verlaten en het vervangen door het idee van meervoudige moderniteiten, wat ons in staat zal stellen om het belang van verschillende culturele achtergronden te erkennen en op de juiste waarde te schatten. Met het idee van meervoudige moderniteiten in plaats van de singulaire verken ik het Confucianistische concept van 'zelf' en idee van het goede leven dat 
dit belichaamt. Vergelijkbaar met wat ik heb gedaan in hoofdstuk twee is het doel van dit hoofdstuk om de basis te leggen voor mijn analyse van de prudentiële beoordeling van digitale media in (hedendaags) China door het verkennen van de normatieve en evaluatieve middelen die beschikbaar zijn in de Confucianistische traditie.

Ik heb de Walzeriaanse benadering toegepast in een intra-culturele context in hoofdstuk drie, maar de aanpak is ook bruikbaar in een inter-culturele context. In hoofdstuk vijf heb ik laten zien hoe de Walzeriaanse benadering kan worden toegepast op mondiaal niveau. Ik analyseer de positie van de Chinese Communistische Partij aangaande het internet vanuit het Confucianistische concept van 'zelf', en illustreer de fundamentele rol van Confuciaanse waarden (en het Confuciaanse begrip van het goede leven) in de grondslag van China's internet beleid en in de normatieve oordelen in opiniestukken. Ik stel dat als het inderdaad zo is dat China's Internet wordt gebaseerd is op een ander normatief en axiologisch fundament, namelijk het Confucianistische concept van 'zelf', dat dan ook de vraag 'is het Internet goed of slecht?', of de vraag 'hoe we moeten leven met digitale media? ', anders moet worden beantwoordt-op een manier welke sociaal en cultureel sensitief is. Om dit te illustreren voeg ik een bespreking toe van sociale media vanuit het Confucianistische perspectief. Ik laat zien dat er een prima facie incompatibiliteit bestaat tussen de Confuciaanse manier van leven en sociale media, maar laat ook zien dat de incompatibilieit tussen dezen kan worden opgelost. Kortom, de boodschap van dit hoofdstuk is dat verschillende maatschappijen hun eigen normatieve analyse van digitale media met hun eigen evaluatieve en normatieve middelen nodig hebben.

Impliciet aanwezig in het project van Net recommendation en de Walzeriaanse benadering van digitale media en het goede leven is de verantwoordelijkheid die filosofen hebben om pro-actief praktische aanbevelingen te bieden aan het publiek, dat wil zeggen: gebruikers van digitale media. Het doen van aanbevelingen wordt echter vaak gezien als paternalistisch, en wordt daardoor beschouwd als moreel onwenselijk. Deze kritiek moet weerlegd worden als het project van Net recommendation of de Walzeriaanse benadering beschouwd moet worden als een (moreel) haalbare optie. In hoofdstuk zes beschouw ik deze kritiek nader. Ik stel dat het aanbieden van aanbevelingen inderdaad paternalistisch is, maar dat we dit niet moeten zien als moreel problematisch, omdat paternalisme onvermijdelijk is in onze technologisch-gemedieerde levens. Filosofen zouden dus niet moeten terugschrikken van het doen van aanbevelingen -- vooral als dit alleen is om de zorg over paternalisme. Hoewel er reeds filosofen zijn die de 
onvermijdelijkheid van paternalisme in onze technologisch-gemedieerde levens als serieuze optie zien, wat tot uiting komt in het idee van design ethics, wil ik erop wijzen dat ook dit moet worden aangevuld met het doen van aanbevelingen. Tot slot wil ik laten zien hoe de Walzeriaanse benadering design ethics kan aanvullen, en hoe het daadwerkelijk de zorg voor paternalisme bij het doen van aanbevelingen kan minimaliseren.

Ten slotte bied ik in het epiloog een korte samenvatting van dit onderzoek en zal ik kort ingaan op een aantal praktische implicaties van het project van Net recommendation en de Walzeriaanse benadering.

Tezamen hoop ik dat deze hoofdstukken een adequate illustratie en verdediging bieden - in de vorm van de Walzeriaanse benadering — van het project van Net recommendation, namelijk een evenwichtige en constructieve manier om de relaties tussen digitale media en mensen, en met name de relaties tussen de te onderzoeken digitale media en het goede leven, die niet start met de aanname van de digitale media als bron van morele problemen, en die streeft aanbevelingen voor specifieke manieren om digitale media en digitaal-gemedieerde gebruiken te hervormen en/of transformeren die ons in staat stellen een betere relatie te hebben met digitale media, en ons dus helpen om een daarmee betere levens te leven. 



\section{BIBLIOGRAPHY}

Adams, M. (2003). The Reflexive Self and Culture: A Critique. British Journal of Sociology, 54 (2), 221-238.

- (2006). Hybridizing Habitus and Reflexivity: Towards an Understanding of Contemporary Identity? Sociology, 40 (3), 511-528.

- (2007). Self and Social Change. London: Sage.

Albrechtslund, A. (2007). Ethics and Technology Design. Ethics and Information Technology, 9, 63-72.

- (2008). Online Social Networking as Participatory Surveillance. First Monday [Online], 13 (3), 2 March 2008.

Alcoff, L.M. (2002). Does the Public Intellectual Have Intellectual Integrity? Metaphilosophy 33 (5), 521-534.

Appiah, A. (2005). The Ethics of Identity. Princeton, N.J.: Princeton University Press.

Argyrou, V. (2005). 'Reflexive Modernization' and Other Mythical Realities. Anthropological Theory, 3 (1), 27-41.

Aspects of Network Culture [In CHINESE: 網絡文化面面觀]. In Guangming Daily, 13 May 2010-17 June 2010. Available Online at: http://www.gmw.cn/content/ node_8562.htm

BAI, T. (2008). A Mencian Version of Limited Democracy. Res Publica, 14 (1), 19-34.

Bakardjieva, M., \& Gaden, G. (2011). Web 2.0 Technologies of the Self. Philosophy \& Technology. DOI: 10.1007/s13347-011-0032-9

Ballantyne, G. (2008). Unification or Multiplication?: Theorizing Multiple Modernities. The International Journal of the Humanities, 5 (10), 53-59.

Barbrook, R. (2007). Imaginary Futures: From Thinking Machines to the Global Village. London: Pluto.

Barnes, S.B. (2006). A Privacy Paradox: Social Networking in the United States. First Monday [Online], 11 (9), 4 September 2006.

Barry, B. (1990). Social Criticism and Political Philosophy. Philosophy and Public Affairs, 19 (4), 360-373.

Beck, U. (1992). Risk Society: Towards a New Modernity. London: Sage.

Beck, U., \& Beck-Gernsheim, E. (2002). Individualization: Institutionalized Individualism and Its Social and Political Consequences. London: Sage.

Beck, U., \& Lau, C. (2005). Second Modernity as a Research Agenda: Theoretical and Empirical Explorations in the 'Meta-change' of Modern Society. British Journal of Sociology, 56 (4), 525-557. 
Beck, U., Bonss, W., \& Lau, C. (2003). The Theory of Reflexive Modernization: Problematic, Hypotheses and Research Programme. Theory, Culture \& Society, 20 (2), 1-33.

Beck, U., Giddens, A., \& Lash, S. (1994). Reflexive Modernization: Politics, Tradition, and Aesthetics in the Modern Social Order. Cambridge: Polity Press.

Beer, D., \& Burrows, R. (2007). Sociology and, of and in Web 2.0: Some Initial Considerations. Sociological Research Online, 12 (5), 30 September 2007. Available Online at: http://www.socresonline.org.uk/12/5/17.html

Bell, D., Loader, B.D., Pleace, N., \& Schuler, D. (2004). Cyberculture: the Key Concepts. New York: Routledge.

Bell, D.A. (2010). Communitarianism. In E. Zalta (ed.), The Stanford Encyclopedia of Philosophy (Fall 2010 Edition). Available Online at: http://plato.stanford.edu/ archives/fall2010/entries/communitarianism/

Benkler, Y. (2006). The Wealth of Networks: How Social Production Transforms Markets and Freedom. New Haven: Yale University Press.

Berger, P.L., \& Huntington, S.P. (eds.) (2002). Many Globalizations: Cultural Diversity in the Contemporary World. Oxford: Oxford University Press.

Bhambra, G.K. (2007). Rethinking Modernity: Postcolonialism and the Sociological Imagination. New York: Palgrave.

Bijker, W.E. (2003). The Need for Public Intellectuals: A Space for STS. Science, Technology, \& Human Values, 28 (4), 443-450.

Bilton, N. (2010). I Live in the Future \& Here's How It Works: Why Your World, Work, and Brain are Being Creatively Disrupted. New York: Crown Business.

Birnbacher, D. (1999). Ethics and Social Science: Which Kind of Co-Operation? Ethical Theory and Moral Practice, 2 (4), 319-336.

Bockover, M. (2003). Confucian Values and the Internet: A Potential Conflict. Journal of Chinese Philosophy, 30 (2), 159-175.

- (2010). Confucianism and Ethics in the Western Philosophical Tradition I: Foundational Concepts. Philosophy Compass, 5 (4), 307-316.

Borgmann, A. (1984). Technology and the Character of Contemporary Life: A Philosophical Inquiry. Chicago: University of Chicago Press.

- (1999). Holding on to Reality: The Nature of Information at the Turn of the Millennium. Chicago: University of Chicago Press.

boyd, d. (2010a). Social Network Sites as Networked Publics: Affordances, Dynamics, and Implications. In Z. Papacharissi (ed.), Networked Self: Identity, Community, and Culture on Social Network Sites (pp. 39-58). New York: Routledge

—. (2010b). Social Steganography: Learning to Hide in Plain Sight. Digital Media and Learning Blog, 23 August 2010. Available Online at: http://dmlcentral.net/ blog/danah-boyd/social-steganography-learning-hide-plain-sight

boyd, d., \& Ellison, N.B. (2008). Social Network Sites: Definition, History, and Scholarship. Journal of Computer-Mediated Communication, 13, 210-230.

Bransen, J. (2000). Alternatives of Oneself: Recasting Some of Our Practical Problems. Philosophy and Phenomenological Research, 60 (2), 381-400.

— (2002). Making and Finding Oneself. In A.W. Musschenga, W. van Haaften, B. Spiecker \& M. Slors (eds.), Personal and Moral Identity (pp. 77-96). Dordrecht: Kluwer. 
- (2008). Personal Identity Management. In K. Atkins \& C. Mackenzie (eds.), Practical Identity and Narrative Agency (pp. 101-120). New York: Routledge.

Brey, P. (2000). Disclosive Computer Ethics. ACM SIGCAS Computers and Society, 30 (4), 10 16.

— (2003). Theorizing Modernity and Technology. In T. Misa, P. Brey \& A. Feenberg (eds.), Modernity and Technology (pp. 33-71). Cambridge, Mass.: MIT Press.

- (2006). Evaluating the Social and Cultural Implications of the Internet. ACM SIGCAS Computer \& Society, 36 (3), 41-48.

— (2007). Theorizing the Cultural Quality of New Media. Techné: Research in Philosophy and Technology, 11 (1), 1-18.

- (2010a). Values in Technology and Disclosive Computer Ethics. In L. Floridi (ed.), The Cambridge Handbook of Information and Computer Ethics (pp. 41-58). Cambridge: Cambridge University Press.

- (2010b). Philosophy of Technology after the Empirical Turn. Techné: Research in Philosophy and Technology, 14 (1), 36-48.

Burawoy, M. (2005). For Public Sociology. American Sociological Review, 70, 4-28.

Bynum, T. (2008). Computer and Information Ethics. In E. Zalta (ed.), The Stanford Encyclopedia of Philosophy (Winter 2008 Edition). Available Online at: http://plato.stanford.edu/archives/win2008/entries/ethics-computer/

Carens, J. (2000). Culture, Citizenship, and Community: A Contextual Exploration of Justice as Evenhandedness. New York: Oxford University Press.

- (2004). A Contextual Approach to Political Theory. Ethical Theory and Moral Practice, 7 (2), 117-132.

Carr, N. (2008). Is Google Making Us Stupid. The Atlantic, July/August 2008. Available Online at: http://www.theatlantic.com/doc/200807/google

—. (2010). The Shallows: What the Internet is Doing to Our Brains. New York: W.W. Norton.

Chan, J. (2002). Moral Autonomy, Civil Liberties, and Confucianism. Philosophy East and West, 52 (3), pp. 281-310.

Chan, W.-T. (trans.) (1969). A Source Book in Chinese Philosophy. Princeton, N.J.: Princeton University Press.

Cheung, T.S., Chan, H.M., Chan, K.M., King, A.Y.C., Chiu, C.Y., \& Chung, F.Y. (2003). On Zhongyong Rationality: The Confucian Doctrine of the Mean as a Missing Link between Instrumental Rationality and Communicative Rationality. Asian Journal of Social Science, 31 (1), 107-127.

- (2006). How Confucian Are Contemporary Chinese? Construction of an Ideal Type and Its Application to Three Chinese Communities. European Journal of East Asian Studies, 5 (2), 157-180.

Christman, J. (2009). The Politics of Persons: Individual Autonomy and Socio-historical Selves. Cambridge: Cambridge University Press.

Cline, E.M. (2009). The Way, the Right, and the Good. Journal of Religious Ethics, 37 (1), 107 129.

Clinton, H. (2010). Internet Freedom. Foreign Policy, 21 January 2010. Available Online at: http://www.foreignpolicy.com/articles/2010/01/21/internet_freedom 
Cowley, C. (2005). A New Rejection of Moral Expertise. Medicine, Health Care and Philosophy, 8, 273-279.

Coyne, R. (1999). Technoromanticism: Digital Narrative, Holism, and the Romance of the Real. Cambridge, Mass.: MIT Press.

Crisp, R. (2008). Well-Being. In E. Zalta (ed.), The Stanford Encyclopedia of Philosophy (Winter 2008 Edition). Available Online at: http://plato.stanford.edu/archives/ win2008/entries/well-being/

Cummings, M.L. (2006). Integrating Ethics in Design through the Value-Sensitive Design Approach. Science and Engineering Ethics, 12, 701-715.

Dahlberg, L. (2010). Cyber-libertarianism 2.0: A Discourse Theory/Critical Political Economy Examination. Cultural Politics, 6 (3), 331-356.

Damm, J. (2007). The Internet and the Fragmentation of Chinese Society. Critical Asian Studies, 39 (2), 273-294.

Daniels, N. (2011). Reflective Equilibrium. In E. Zalta (ed.), The Stanford Encyclopedia of Philosophy (Spring 2011 Edition). Available Online at: http://plato.stanford.edu/ archives/spr2011/entries/reflective-equilibrium/

Davies, G. (2007). Worrying about China: The Language of Chinese Critical Inquiry. Cambridge, Mass.: Harvard University Press.

- (2010). Affirming the Human in China. boundary 2, 37 (1), 57-90.

Delanty, G. (2006). Modernity and the Escape from Eurocentrism. In G. Delanty (ed.), Handbook of Contemporary European Social Theory (pp. 266-276). London: Routledge.

Deuze, M. (2009). Media Industries, Work and Life. European Journal of Communication, 24 (4), 1-14.

—. (2011). Media Life 2.0. Media, Culture \& Society, 33 (1), 137-148.

—. (forthcoming). Media Life. Cambridge: Polity Press.

Dupre, L.K. (2004). The Enlightenment and the Intellectual Foundations of Modern Culture. New Haven: Yale University Press.

Dworkin, G. (2010). Paternalism. In E. Zalta (ed.), The Stanford Encyclopedia of Philosophy (Summer 2010 Edition). Available Online at: http://plato.stanford.edu/archives/ sum2010/entries/paternalism/

Ess, C. (2009). Digital Media Ethics. Cambridge: Polity.

- (2010). The Embodied Self in a Digital Age: Possibilities, Risks, and Prospects for a Pluralistic (Democratic/Liberal) Future? Nordicom Information, 32 (2/3), pp. 105118.

- (2011). Self, Community, and Ethics in Digital Mediatized Worlds. In C. Ess \& M. Thorseth (eds.), Trust and Virtual worlds: Contemporary Perspectives (pp. 3-30). New York, Peter Lang.

Fallis, D. (2009). Introduction: The Epistemology of Mass Collaboration. Episteme, 6, 1-7.

FAN, R. (2010). Reconstructionist Confucianism: Rethinking Morality after the West. New York: Springer.

Feenberg, A. (1995). Alternative Modernity: The Technical Turn in Philosophy and Social Theory. Berkeley: University of California Press

Feinberg, J. (1986). Harm to Self. New York: Oxford University Press.

Finlay, S. (2005). Value and Implicature. Philosophers' Imprint, 5 (4), July 2005. Available Online at: http://www.philosophersimprint.org/005004/ 
Flichy, P. (2007). The Internet Imaginaire. Cambridge, Mass.: MIT Press.

Floridi, L. (ed.) (2010). The Cambridge Handbook of Information and Computer Ethics. Cambridge: Cambridge University Press.

Frankfurt, H.G. (1988). The Importance of What We Care About. In The Importance of What We Care About: Philosophical Essays. Cambridge: Cambridge University Press.

- (1999a). Necessity, Volition, and Love. Cambridge: Cambridge University Press.

_ (1999b). On the Necessity of Ideals. In Necessity, Volition, and Love. Cambridge: Cambridge University Press.

- (2004). The Reasons of Love. Princeton, N.J.: Princeton University Press.

—. (2006). Taking Ourselves Seriously \& Getting It Right. Stanford, Calif.: Stanford University Press.

Frick, M.-L., \& Oberprantacher, A. (2011). Shared is not Yet Sharing, Or: What Makes Social Networking Services Public? International Review of Information Ethics, 15, 17-23.

Friedman, B., \& Kahn, P.H. (2007). Human Values, Ethics, and Design. In A. Sears \& J.A. Jacko (eds.), The Human-Computer Interaction Handbook: Fundamentals, Evolving Technologies, and Emerging Applications, 2nd Edition (pp. 1241-1266). Boca Raton, FL: CRC Press.

Friedman, B., Kahn, P.H., \& Borning, A. (2008). Value Sensitive Design and Information Systems. In K.E. Himma and H.T. Tavani (eds.), Handbook of Information and Computer Ethics (pp. 69-108). Hoboken, NJ: John Wiley \& Sons

Fuchs, C. (2010). Social Software and Web 2.0: Their Sociological Foundations and Implications. In S. Murugesan (ed.), Handbook of Research on Web 2.0, 3.0, and X.0: Technologies, Business, and Social Applications, Vol. II (pp. 764-789). Hershey, PA: IGIGlobal.

Fuchs, C., Hofkirchner, W., Schafranek, M., Raffl, C., Sandoval, M., \& Bichler, R. (2010). Theoretical foundations of the Web: Cognition, Communication, and Co-operation: Towards an Understanding of Web 1.0, 2.0, 3.0. Future Internet, 2 (1), 41-59.

Fukuyama, F. (1992). The End of History and the Last Man. New York: Free Press.

Fuller, S. (2004). Intellectuals: An Endangered Species in the Twenty-first Century? Economy and Society, 33 (4), 463-483.

GAO, L. (2008). Moral Revolution in the May 4th Movement and the Confucian Moral Tradition [In CHINESE: 五四倫理革命與儒家德性傳統]. Twenty-first century [Online], 72, 31 March 2008. Available Online at: http://www.cuhk.edu.hk/ics/21c/ supplem/essay/9903015.htm

Gaonkar, D.P. (1999). On Alternative Modernities. Public Culture, 11 (1), 1-18.

Garren, D.J. (2006). Paternalism, Part I. Philosophical Books, 47 (4), 334-341.

—. (2007). Paternalism, Part II. Philosophical Books, 48 (1), 50-59.

Gasper, D. (2006). Human Well-Being: Concepts and Conceptualizations. In M. McGillivray (ed.), Human Well-Being: Concept and Measurement (pp. 23-64). Basingstoke, UK: Palgrave MacMillan.

Giddens, A. (1990). The Consequences of Modernity. Stanford, Calif.: Stanford University Press.

- (1991). Modernity and Self-identity: Self and Society in the Late Modern Age. Stanford, Calif.: Stanford University Press. 
Ginnell, C.K. (2009). From Consumer to Prosumer to Produser: Who Keeps Shifting My Paradigm? (We Do!). Public Culture, 21 (3), 577-598.

Glynos, J., and Howarth, D., Norval, A., \& Speed, E. (2009). Discourse Analysis: Varieties and Methods. ESRC National Centre for Research Methods Review Paper. Available Online at: http://eprints.ncrm.ac.uk/796/

Goldman, A.I. (2008). The Social Epistemology of Blogging. In J. van den Hoven and J. Weckert (eds.), Information Technology and Moral Philosophy (pp. 111-122). Cambridge, Cambridge University Press.

Graham, A. C. (1989). Disputers of the Tao: Philosophical Argument in Ancient China. La Salle, Ill.: Open Court.

Graham, P. (2005). Web 2.0. Paul Graham's website, November 2005. Available Online at: http://paulgraham.com/web20.html

Great Firewall of China (2011). In GMH Lexicon, China Digital Times. Available Online at: http://chinadigitaltimes.net/space/Great_Firewall_of_China

Griffin, J. (1996). Value Judgement: Improving Our Ethical Beliefs. Oxford: Clarendon Press.

Grill, K. (2012). Paternalism. In R. Chadwick, R (ed.), Encyclopedia of Applied Ethics, $2^{\text {nd }}$ Edition (pp. 359-369). San Diego: Academic Press.

Gu, H. (2001). On Three Approaches to Modern Individualised Personality Research [In CHINESE: 論近代個性化人格研究中的三種路向]. Social Science Journal, 133, 37-42.

Hall, D.L., \& Ames, R.T. (1987) Thinking through Confucius. Albany: State University of New York Press.

Hansen, C. (1992). A Daoist theory of Chinese Thought: A Philosophical Interpretation. New York: Oxford University Press.

Haybron, D.M. (2008). The Pursuit of Unhappiness: The Elusive Psychology of Well-being. Oxford: Oxford University Press.

Haybron, D.M., \& Tiberius, V. (2012). Normative Foundations for Well-Being Policy. Papers on Economics \& Evolution. Available Online at: http://www.econ.mpg.de/english/ research/EVO/discuss.php\#p2012-02

Herold, D.K. (2011). Conclusion: Netizens and Citizens, Cyberspace and Modern China. In D. K. Herold \& P. Marolt (eds.), Online Society in China: Creating, Celebrating, and Instrumentalising the Online Carnival (pp. 200-208). New York: Routledge.

Higgs, E., Light, A., \& Strong, D. (eds.) (2000). Technology and the Good Life? Chicago: University of Chicago Press.

Himma, K.E., \& Tavani, H.T. (eds.) (2008). The Handbook of Information and Computer Ethics. Hoboken, N.J.: Wiley.

Honneth, A. (2008). Reification: A New Look at an Old Idea. Oxford: Oxford University Press.

HuANG, Y. (2005). Some Fundamental Issues in Confucian Ethics: A Selective Review of Encyclopedia of Chinese Philosophy. Journal of Chinese Philosophy, 32 (3), 509-528.

—. (2007). Confucian Theology: Three Models. Religion Compass, 1 (4), 455-478.

Hull, G., Lipford, H.R., \& Latulipe, C. (2011). Contextual Gaps: Privacy Issues on Facebook. Ethics and Information Technology, 13 (4), 289-302.

Hunston, S., \& Thompson, G. (2006). Evaluation in Text. In K. Brown (ed.), The Encyclopedia of Language and Linguistics, $2^{\text {nd }}$ Edition, Vol. 4 (pp. 305-312). Oxford: Elsevier.

Huntington, S.P. (1996). The Clash of Civilizations and the Remaking of World Order. New York: Simon \& Schuster. 
Hurka, T. (2006). Value-Theory. In D. Copp (ed.), The Oxford Handbook of Ethical Theory (pp. 357-79). New York: Oxford University Press.

Hwang, K.-K. (2001). The Deep Structure of Confucianism: a Social Psychological Approach. Asian Philosophy, 11 (3), 179-204.

Inglehart, R. (1990). Culture Shift in Advanced Industrial Society. Princeton, N.J.: Princeton University Press.

—. (1997). Modernization and Postmodernization: Cultural, Economic, and Political Change in 43 Societies. Princeton, N.J.: Princeton University Press.

Inglehart, R., \& Norris, P. (2003). Rising Tide: Gender Equality and Cultural Change around the World. Cambridge, UK; New York: Cambridge University Press.

—. (2004). Sacred and Secular: Religion and Politics Worldwide. Cambridge, UK; New York: Cambridge University Press.

Inglehart, R., \& Welzel, C. (2005). Modernization, Cultural Change, and Democracy: The Human Development Sequence. Cambridge, UK; New York: Cambridge University Press.

Introna, L. (2008). Phenomenological Approaches to Ethics and Information Technology. In E. Zalta (ed.), The Stanford Encyclopedia of Philosophy (Winter 2008 Edition). Available Online at: http://plato.stanford.edu/archives/win2008/entries/ethics-itphenomenology/

IP, P.K. (2011). Concepts of Chinese Folk Happiness. Social Indicators Research, 104 (3), 459-474.

Ivanhoe, P.J. (2007). Heaven as a Source for Ethical Warrant in Early Confucianism. Dao, 6 (3), 211-220.

Iversen, O.S., Halskov, K., \& Leong, T.W. (2010). Rekindling Values in Participatory Design. In Proceedings of the 11th Biennial Participatory Design Conference (pp. 91-100), November 2010. Sydney, Australia.

Jacoby, R. (2000). The Last Intellectuals: American Culture in the Age of Academe, 2000 Edition. New York: Basic Books.

—. (2009). Last Thoughts on The Last Intellectuals. Society, 46 (1), 38-44.

JIANG, M. (2010). Authoritarian Informationalism: China's Approach to Internet Sovereignty. SAIS Review, 30 (2), 71-89.

JIN, G. (1997). Chinese Culture and the Consciousness of Rationalizing Common Knowledge [In CHINESE: 中國文化的常識合性精神]. Journal of Chinese Studies, 37, 457-472.

Jin, G., \& Liu, Q. (2009). Studies in the History of Ideas: The Formation of Important Modern Chinese Political Terms [In CHINESE: 觀念史研究 : 中國現代重要政治術語的形成]. Beijing: Law Press China.

Kaya, I. (2004). Modernity, Openness, Interpretation: A Perspective on Multiple Modernities. Theory and Methods, 43 (1), 35-57.

Keen, A. (2007). The Cult of Amateur: How Today's Internet is Killing Our Culture. New York: Doubleday/Currency.

KIM, S. (2009). Self-Transformation and Civil Society: Lockean vs. Confucian. Dao, 8, 383401.

— (2010). Beyond Liberal Civil Society: Confucian Familism and Relational Strangership. Philosophy East and West, 60(4), 476-498. 
- (2011). The Anatomy of Confucian Communitarianism: The Confucian Social Self and Its Discontent. The Philosophical Forum, 42 (2), 111-130.

Korsgaard, C.M. (1996). The Sources of Normativity. Cambridge: Cambridge University Press. Kupperman, J. (1999). Value... and What Follows. New York: Oxford University Press.

- (2010). Confucian Civility, Dao, 9 (1), 11-23.

Lai, K. (2006). Learning from Chinese Philosophies: Ethics of Interdependent and Contextualised Self. UK: Ashgate.

- (2009). Judgment in Confucian Ethics. Sophia, 48, 77-84.

Lanier, J. (2010). You Are Not a Gadget: A Manifesto. London: Allen Lane.

Last, J.V. (2010). Future Guy. The Weekly Standard, 16 (8), 8 November 2010. Available Online at: http://www.weeklystandard.com/articles/future-guy_513321.html

Leadbeater, C. (2008). We-Think. London: Profile.

Legge, J. (trans.) (1861). The Chinese Classics, with a Translation, Critical and Exegetical Notes, Prolegomena, and Copious Indexes, Vol. 1. Hong Kong; London: Trübner.

Lessig, L. (2007). Keen's “The Cult of the Amateur”: BRILLIANT! Lessig Blog, 31 May 2007. Available Online at: http://lessig.org/blog/2007/05/keens_the_cult_of_ the_amateur.html

Levy, J.T. (2007). Contextualism, Constitutionalism, and Modus Vivendi Approaches. In A. Laden \& D. Owen (eds.), Multiculturalism and Political Theory (pp. 173-197).

Cambridge: Cambridge University Press. NY.

LI, C. (2006). The Confucian Ideal of Harmony. Philosophy East and West, 56 (4), 583-603.

Light, B., \& McGrath, K. (2010). Ethics and Social Networking Sites: A Disclosive Analysis of Facebook. Information Technology \& People, 23 (4), 290-311.

Lister, M., Dovey, J., Giddings, S., Grant, I., \& Kelly, K. (2009). New Media: A Critical Introduction. New York: Routledge.

LiU, J. (2007). Confucian Moral Realism. Asian Philosophy, 17 (2), 167-184.

—. (2009). Converting Chinese Philosophy into the Analytic Context. Available Online at: http://faculty.fullerton.edu/jeelooliu/Liu_Analytic-Chinese.pdf

LiU, L. (2006). Quality of Life as a Social Representation in China: A Qualitative Study. Social Indicators Research, 75 (2), 217-240.

- (2008). To Have and To Be: Towards the Social Representation of Quality of Life in China. Journal of Community \& Applied Social Psychology, 18, 233-252

LIU, X.-H. (2004). The Transition of the Conception of Individual: An Important Issue in Studies on China's Modernity [In CHINESE: 個人觀轉型：中國現代性研究中的一個重 要問題]. Journal of East China Normal University (Philosophy and Social Sciences), 136 (16), 35-41.

Liv, Y. (2004). The Self and Li in Confucianism. Journal of Chinese Philosophy, 31 (3), 363376.

Livingstone, S. (2011). Internet, Children and Youth. In M. Consalvo \& C. Ess (eds.), The Handbook of Internet Studies (pp. 348-368). Malden, MA.: Wiley-Blackwell.

Lovink, G. (2002). Dark Fiber: Tracking Critical Internet Culture. Cambridge, MA: MIT Press.

- (2008). Zero Comments: Blogging and Critical Internet Culture. New York: Routledge.

—. (2010). MyBrain.net: the Colonization of Real-time and Other Trends in Web 2.0. Eurozine, 18 March 2010. Available Online at: http://www.eurozine.com/articles/ 2010-03-18-lovink-en.html 
—. (2011/2003). My First Recession: Critical Internet Culture in Transition. Amsterdam: Institute of Network Cultures.

Lu, L. (2001). Understanding Happiness: A Look into the Chinese Folk Psychology. Journal of Happiness Studies, 2, 407-432.

- (2005). In Pursuit of Happiness: The Cultural Psychological Study of SWB. Chinese Journal of Psychology, 47 (2), 99-112.

- (2008a). The Individual-Oriented and Social-Oriented Chinese Bicultural Self: Testing the Theory. The Journal of Social Psychology, 148 (3), 347-373.

—. (2008b). Culture, Self and Subjective Well-Being: Cultural Psychological and Social Change Perspectives. Psychologia, 51, 290-303.

Lu, L., \& Gilmour, R. (2006). Individual-oriented and Socially-oriented Cultural Conceptions of Subjective Well-being: Conceptual Analysis and Scale Development. Asian Journal of Social Psychology, 9, 36-49.

Lu, L., KaO, S.-F., Chang, T.-T., Wu, H.-P., \& ZHANG, J. (2008). The Individual- and Socialoriented Chinese Bicultural Self: A Subcultural Analysis Contrasting Mainland Chinese and Taiwanese. Social Behavior and Personality, 36 (3), 337-346.

Mallan, K., \& Giardina, N. (2009) Wikidentities: Young People Collaborating on Virtual Identities in Social Network Sites. First Monday [Online], 14 (6), 1 June 2009.

Manders-Huits, N. (2011). What Values in Design? The Challenge of Incorporating Moral Values into Design. Science and Engineering Ethics, 17 (2), 271-287.

Martin, J.R., \& White, P.R.R. (2005). The Language of Evaluation: Appraisal in English. Basingstoke/New York: Palgrave Macmillan.

Martinelli, A. (2005). Global Modernization: Rethinking the Project of Modernity. London: Sage.

MenG, B. (2010). Moving Beyond Democratization: A Thought Piece on the China Internet Research Agenda. International Journal of Communication, 4, 501-508.

Molewijk, B., Stiggelbout, A.M., Otten, W., Dupuis, H.M., \& Kievit, J. (2004). Empirical Data and Moral Theory. A Plea for Integrated Empirical Ethics. Medicine, Health Care and Philosophy, 7 (1), 55-69.

Moody-Adams, M.M. (1999). The Idea of Moral Progress. Metaphilosophy, 30 (3), 168-185.

Mosco, V. (2004). The Digital Sublime: Myth, Power, and Cyberspace. Cambridge, Mass.: MIT Press.

Muller, M.J. (2007). Participatory Design: The Third Space in HCI. In A. Sears \& J.A. Jacko (eds.), The Human-Computer Interaction Handbook: Fundamentals, Evolving Technologies, and Emerging Applications, 2nd Edition (pp. 1061-1081). Boca Raton, FL: CRC Press

Muller, M.J., \& Kuhn, S. (1993). Participatory Design. Communications of the ACM, 36 (6), 2428.

Musschenga, A.W. (2005). Empirical Ethics, Context-Sensitivity, and Contextualism. Journal of Medicine and Philosophy, 30 (5), 467-490.

Nuyen, A.T. (1999). Chinese Philosophy and Western Capitalism. Asian Philosophy, 9 (1), 71-79.

- (2007). Confucian Ethics as Role-Based Ethics. International Philosophical Quarterly, 47 (3), 315-328. 
—. (2009). Moral Obligation and Moral Motivation in Confucian Role-Based Ethics. Dao, 8 (1), 1-11.

Oommen, T.K. (1999). Recognizing Multiple Modernities: A Prelude to Understanding Globalization. Available Online at: http://www.social-theory.eu/texts/oommen1999_modernities.pdf

O'Reilly, T. (2005a). What is Web 2.0. O'Reilly Media, 30 September 2005. Available Online at: http://oreilly.com/web2/archive/what-is-web-20.html

—. (2005b). Web 2.0: Compact Definition?. O'Reilly Radar, 1 October 2005. Available Online at: http://radar.oreilly.com/2005/10/web-20-compact-definition.html

Papacharissi, Z., \& Gibson, P.L. (2011). Fifteen Minutes of Privacy: Privacy, Sociality an Publicity on Social Network Sites. In L. Reinecke \& S. Tepte, (eds.), Privacy Online: Theoretical Approaches and Research Perspectives on the Role of Privacy in the Social Web (pp. 75-89). New York: Springer

Perry, E.J. (2008). Chinese Conceptions of "Rights": From Mencius to Mao-and Now. Perspectives on Politics, 6, 37-50.

Platform (2011). In Oxford English Dictionary (Third Edition, June 2006; Online Version, September 2011). Available Online at: http://oed.com/view/Entry/145374

Portal (2011). In Oxford English Dictionary (Third edition, December 2006; Online Version, September 2011). Available Online at: http://oed.com/view/Entry/148114

Postman, N. (1992). Technopoly: the Surrender of Culture to Technology. New York: Knopf. Rawls, J. (1971). A Theory of Justice. Cambridge, Mass.: Harvard University Press.

- (1993). Political Liberalism. New York: Columbia University Press.

Regan, D. (2004). Why am I My Brother's Keeper? In R. J. Wallace, P. Pettit, S. Scheffler \& M. Smith (eds.), Reason and Value: Themes from the Moral Philosophy of Joseph Raz (pp. 202-230). Oxford: Clarendon Press.

Ritzer G., \& Jurgenson, N. (2010). Production, Consumption, Prosumption: The Nature of Capitalism in the Age of the Digital 'Prosumer'. Journal of Consumer Culture, 10, 13-36.

Rosati, C. (2006). Personal Good. In T. Horgan \& M. Timmions (eds.), Metaethics After Moore (pp. 107-131). Oxford: Oxford University Press.

—. (2009). Relational Good and the Multiplicity Problem. Philosophical Issues, 19, 205234.

Schmidt, V.H. (2006). Multiple Modernities or Varieties of Modernity. Current Sociology, 54 (1), 77-97.

Schroeder, M. (2008). Value Theory. In E. Zalta (ed.), The Stanford Encyclopedia of Philosophy (Fall 2008 Edition). Available Online at: http://plato.stanford.edu/ archives/fall2008/entries/value-theory/

Scoccia, D. (2008). In Defence of Hard Paternalism. Law and Philosophy, 27, 351-381.

Seigel, J. (2005). The Idea of the Self: Thought and Experience in Western Europe Since the Seventeenth Century. Cambridge, UK; New York: Cambridge University Press.

Shafer, J. (2010). Digital Native Calms the Anxious Masses. Slate, 13 September, 2010. Available Online at: http://www.slate.com/id/2267161

Shirky, C. (2009). Here Comes Everybody: How Change Happens When People Come Together. London: Penguin.

— (2010). Cognitive Surplus: Creativity and Generosity in a Connected Age. London, Allen Lane. 
SHu, X., \& Zhu, Y. (2009). The Quality of Life in China. Social Indicators Research, 92 (2), 191225.

Sim, M. (2004). A Confucian Approach to Human Rights. History of Philosophy Quarterly, 21 (4), 337-356.

Slingerland, E. (trans.) (2003). Confucius Analects: with Selections from Traditional Commentaries. Indianapolis, Ind.: Hackett Pub. Co.

Song, F.W. (2010). Theorizing Web 2.0: A Cultural Perspective. Information, Communication \& Society, 13 (2), 249-275.

Sreenivasan, G. (1998). Interpretation and Reason. Philosophy and Public Affairs, 27 (2), 142-171.

State Council Information Office of the People's Republic of China [SCIO] (2010). The Internet in China. Xinhua, 8 June 2010. Available Online at: http://news.xinhuanet.com/english2010/china/2010-06/08/c_13339232.htm

Steinkamp, N.L., Gordijn, B., \& ten Have, H.A.M.J. (2008). Debating Ethical Expertise. Kennedy Institute of Ethics Journal, 18 (2), 173-192.

Street, S. (forthcoming). Objectivity and Truth: You'd Better Rethink It. Philosophy \& Public Affairs. Available Online at: http://files.nyu.edu/jrs477/public/Sharon\%20Street $\% 20$ -\%200bjectivity\%20and\%20Truth.pdf

Stevens, S.M. (2008). Speaking Out: Toward an Institutional Agenda for Refashioning STS Scholars as Public Intellectuals. Science, Technology, \& Human Values, 33 (6), 730-753.

Sunstein, C. (2006). Infotopia: How Many Minds Produce Knowledge. New York: Oxford University Press.

Szablewicz, M. (2010). The Ill Effects of Opium for the Spirit: A Critical Cultural Analysis of China's Internet Addiction Moral Panic. Chinese Journal of Communication, 3(4), 453470.

Tapscott, D., \& Williams, A.D. (2006). Wikinomics: How Mass Collaboration Changes Everything. New York: Portfolio.

- (2010). Macrowikinomics: Rebooting Business and the World. New York: Portfolio.

Taylor, C. (1989). Sources of the Self: The Making of Modern Identity. Cambridge: Cambridge University Press.

- (1999). Two Theories of Modernity. Public Culture, 11 (1), 153-174.

Thaler, R.H., \& Sunstein, C. (2008). Nudge: Improving Decisions about Health, Wealth, and Happiness. New Haven: Yale University Press.

Thierer, A. (2011). The Case for Internet Optimism, Part 1: Saving the Net from Its Detractors. In B. Szoka \& A. Marcus (eds.), The Next Digital Decade: Essays on the Future of the Internet (pp. 57-87). Washington: TechFreedom.

Thomson, J.J. (1997). The Right and the Good. Journal of Philosophy, 94 (6), 273-298.

Tiberius, V. (2004). Cultural Differences and Philosophical Accounts of Well-being. Journal of Happiness Studies, 5, 293-314.

—. (2006). Well-Being: Psychological Research for Philosophers. Philosophy Compass, 1 (5), 493-505.

Tiwald, J. (2010). Confucianism and Virtue Ethics: Still a Fledgling in Chinese and Comparative Philosophy. Comparative Philosophy, 1 (2), 55-63.

Tu, W. (1993). Confucianism. In A. Sharma (ed.), Our Religions: The Seven World Religions Introduced (141-227). San Francisco: Harper. 
Turkle, S. (2011). Alone together: Why We Expect More from Technology and Less from Each Other. New York: Basic Books.

Vallor, S. (2010). Social Networking Technology and the Virtues, Ethics and Information Technology, 12 (2), 157-170.

Van De Veer, D. (1979). Paternalism and Subsequent Consent. Canadian Journal of Philosophy, 9 (4), 631-642.

van den Hoven, J. (2008). Moral Methodology and Information Technology. In K.E. Himma \& H.T. Tavani (eds.), Handbook of Information and Computer Ethics (pp. 49-67). Hoboken, NJ.: John Wiley \& Sons

van den Hoven, J., \& Weckert, J. (eds.) (2008). Information Technology and Moral Philosophy. Cambridge: Cambridge University Press.

Van Dijck, J., \& Nieborg, D. (2009). Wikinomics and Its Discontents: A Critical Analysis of Web 2.0 Business Manifestos. New Media \& Society, 11 (5), 855-874.

Van Norden, B. (2000). Confucius and the Analects: New Essays. Oxford: Oxford University Press.

—. (trans.) (2001). Mengzi (Mencius). In P. Ivanhoe \& B. van Norden (eds.), Readings in Classical Chinese Philosophy (pp. 115-159). New York, NY: Seven Bridges Press.

Velleman, J.D. (2000) Well-being and Time. In The Possibility of Practical Reason. Oxford: Oxford University Press.

Venn, C., \& Feaherstone, M. (2006). Modernity. Theory, Culture \& Society, 23 (2-3), 457-476.

Verbeek, P.P.C.C. (2002). Devices of Engagement: On Borgmann's Philosophy of Information and Technology. Techné: Research in Philosophy and Technology, 6 (1), 69-92.

- (2005). What Things Do: Philosophical Reflection on Technology, Agency, and Design. PA: Pennsylvania State University.

- (2006). Materializing Morality: Design Ethics and Technological Mediation. Science, Technology, \& Human Values, 31 (3), 361-380.

- (2008). Morality in Design: Design Ethics and the Morality of Technological Artifacts. In P. Vermass, P. Kroes, A. Light \& S.A. Moore (eds.), Philosophy and Design: from Engineering to Architecture (pp. 91-103). Dordrecht: Springer.

- (2009). Cultivating Humanity: Towards a Non-Humanist Ethics of Technology. In S. Riis, J.K.B. Olsen \& E. Selinger (eds.) New Waves in Philosophy of Technology. Basingstoke (pp. 254-260). New York: Palgrave Macmillan.

- (2010). Accompanying Technology. Techné: Research in Philosophy and Technology, 14 (1), 49-54.

Wagner, P. (1994). Liberty and Discipline: A Sociology of Modernity. London: Routledge.

—. (2009). Modernity as Experience and as Interpretation: Towards Something Like a Cultural Turn in the Sociology of "Modern Society". In P. Hedström \& B. Wittrock (eds.), Frontiers of Sociology (pp. 245-266). Leiden, Brill.

Walzer, M. (1981). Philosophy and Democracy. Political Theory, 9, 379-399. Reprinted in Walzer (2007).

—. Liberalism and the Art of Separation. Political Theory, 12, 315-330. Reprinted in Walzer (2007).

—. (1985). Interpretation and Social Criticism. The Tanner Lectures on Human Values. Available Online at: http://www.tannerlectures.utah.edu/lectures/documents/ walzer88.pdf 
- (1988). The Company of Critics: Social Criticism and Political Commitment in the Twentieth Century. New York: Basic Books.

- (1989). A Critique of Philosophical Conversation. Philosophical Forum, 21, 182-196. Reprinted in Walzer (2007).

_ (1993). Objectivity and Social Meaning. In M.C. Nussbaum \& A. Sen (eds.), The Quality of Life (pp.165-177). Oxford: Clarendon Press. Reprinted in Walzer (2007).

— (1994). Thick and Thin: Moral Argument at Home and Abroad. Notre Dame: University of Notre Dame Press.

— (2007). Thinking Politically: Essays in Political Theory. New Haven: Yale University Press.

WANG, C. (2010). Concerning the Development and Administration of Our Country's Internet. China Right Forum, 2. Available Online at: http://hrichina.org/content/3241

WANG, K. (2007). A Rediscovery of Heaven-and-Human Oneness. The American Journal of Economics and Sociology, 66 (1), 237-259.

Wang, R. (2010). The Virtuous Body at Work: The Ethical Life as Qi in Motion. Dao, 9, 339351.

Weber, M. (2001/1930). The Protestant Ethic and the Spirit of Capitalism. London: Routledge.

Wedgwood, R. (2009). The "Good" and the "Right" Revisited. Philosophical Perspectives, 23, 499-519.

White, P.R.R. (2002). Appraisal - the Language of Evaluation and Stance. In J. Verschueren, J. Östman, J. Blommaert \& C. Bulcaen (eds.), The Handbook of Pragmatics (pp. 1-27). Amsterdam: John Benjamins.

— (2005). Appraisal: An Overview. Appraisal Website. Available Online at: http://grammatics.com/appraisal/AppraisalGuide/UnFramed/AppraisalOverview.htm

Williams, B. (1985). Ethics and Limits of Philosophy. Cambridge, Mass.: Harvard University Press.

- (2005). Modernity and the Substance of Ethical Life. In In the Beginning was the Deed: Realism and Moralism in Political Argument. Princeton, N.J.: Princeton University Press.

Winner, L. (1986). Do Artifacts Have Politics? In The Whale and the Reactor: A Search for Limits in an Age of High Technology. Chicago: University of Chicago Press.

— (1996). Who Will We Be in Cyberspace? Information Society, 12 (1), 63-72.

- (1997). Cyberlibertarian Myths and the Prospects for Community. ACM SIGCAS Computers and Society, 27 (3), 14-19.

Wong, D. (2004a). Relational and Autonomous Selves. Journal of Chinese Philosophy, 31 (4), 419-432.

- (2004b). Rights and Community in Confucianism. In K.L. Shun \& D. Wong (eds.), Confucian Ethics: a Comparative Study of Self, Autonomy and Community (pp. 31-48). New York: Cambridge University Press.

— (2006). Natural Moralities: A Defense of Pluralistic Relativism. New York: Oxford University Press.

- (2008). Chinese Ethics. In E. Zalta (ed.), The Stanford Encyclopedia of Philosophy (Fall 2008 Edition). Available Online at: http://plato.stanford.edu/archives/fall2008/ entries/ethics-chinese/ 
- (2009). Comparative Philosophy: Chinese and Western. In E. Zalta (ed.), The Stanford Encyclopedia of Philosophy (Winter 2009 Edition). Available Online at:

http://plato.stanford.edu/archives/win2009/entries/comparphil-chiwes/

Wong, P.-H. (2010). Information Technology, the Good and Modernity. In J. Vallverdu (ed.),

Thinking Machines and the Philosophy of Computer Science: Concepts and Principles (pp.

223-237). Hershey, PA: Information Science Reference.

XU, J. (2009). The Dissolution of the Big Self: The Transformation of Individualism in

Modern China [In ChINESE: 大我的消解一一現代中國個人主義思潮的變遷]. Chinese

Social Sciences Quarterly, 26, 1-21. Available Online at: http://www.ias.fudan.edu.cn/

News/Detail.aspx?ID=1184

YAN, Y. (2010). The Chinese Path to Individualization. The British Journal of Sociology, 61 (3), 489-512.

Yanow, D., \& Schwartz-Shea, P. (2006). Introduction. In D. Yanow \& P. Schwartz-Shea (eds.), Interpretation and Method: Empirical Research Methods and the Interpretive Turn (pp. xi-xxvii). New York: M.E. Sharpe.

Yu, E., \& FAN, R. (2007). A Confucian View of Personhood and Bioethics. Bioethical Inquiry, 4, 171-179.

Yu, J. (2005). Confucius' Relational Self and Aristotle's Political Animal. History of Philosophy Quarterly, 22 (4), 281-300.

—. (2008). Soul and Self: Comparing Chinese Philosophy and Greek Philosophy. Philosophy Compass, 3 (4), 604-618.

YU, K.P. (2010). The Confucian Conception of Harmony. In J. Tao, A. Cheung, M. Painter, \& C. Li (eds.), Governance for harmony in Asia and Beyond (pp. 15-36). London: Routledge.

Zimmerman, M.J. (2009). Understanding What is Good for Us. Ethical Theory and Moral Practice, 12 (4), 429-439. 